\title{
Sepsis 2012
}

\author{
Paris, France. 7-9 November 2012
}

Published: 14 November 2012

These abstracts are available online at http://ccforum.com/supplements/16/S3

\section{POSTER PRESENTATIONS}

\section{P1}

Modeling sepsis using neural nets and biomarkers of organ

dysfunction in patients, with links to animal models

J Schentag ${ }^{1 *}$, D Parish², S Opal ${ }^{3}$

${ }^{1}$ University at Buffalo, Buffalo, NY, USA; ${ }^{2}$ CPL Associates LLC, Buffalo, NY, USA;

${ }^{3}$ Brown University, Providence, RI, USA

Critical Care 2012, 16(Suppl 3):P1

Background: Most clinical trials of sepsis treatment modalities fail at their primary objective of establishing superiority over placebo when added to background standard of care. While there is no definitive explanation for the high failure rate, it might be stated that our attempts to insert a new therapeutic agent into standard of care encounters severe problems with definition of exactly what stage is ongoing, and what are the criteria for progression or resolution from that time point onwards. Clearly there is need for a means of defining steps in the septic process that would apply to individuals, and to better define the course of sepsis in each patient after they are enrolled in a trial.

Methods: For core model development, 30 septic patients were studied for time-related progression in relation to biomarkers, employing a Load Model in a neural net algorithm in MatLab. Causative bacterial infections were linked to primary infection sites. In order to minimize overparameterization, the model was allowed to estimate outputs using the best three input parameters. Bacterial load was tracked from origin using clinical and microbiologic data to provide an estimate at the start of sepsis. The bacterial load as well as clinical and laboratory parameters were model inputs with the output parameter being organ failures and/ or mortality.

Results: At onset of sepsis, human bacterial load estimates ranged from between $10^{8}$ and $10^{11} \mathrm{CFU}$, which is consistent with inocula in animal models of sepsis. Sepsis proceeds to organ failures and mortality in a series of steps that are initially linked to bacterial load and inflammatory response, followed by coagulopathy, ischemia, oxygen deprivation in organs and tissues, and culminating in organ failures. The later stages of sepsis are all driven by metabolic parameters, and there seems to be little benefit to blocking inflammation at later stages. Substrate and oxygen deficiencies must be addressed first.

Conclusion: Neural net progression models based on biomarkers and physiological markers are able to describe the evolution of sepsis to septic shock, organ failures, and provide some evidence that mortality may be a consequence of the stages of sepsis. Overall, these models appear useful to the task of sorting out organ failure endpoints and mechanisms in individual patients with sepsis progression across sepsis to septic shock.

\section{P2}

Extracellular matrix turnover, angiogenesis and endothelial function in acute lung injury: relationship to pulmonary dysfunction and outcome

S Sayed", N Idriss, H Sayyed

Faculty of Medicine, Assuit University, Assuit, Egypt

E-mail: 1@bmc.com

Critical Care 2012, 16(Suppl 3):P2

Background: Acute lung injury (ALI) is a syndrome with a diagnostic criteria based on hypoxemia and a classical radiological appearance, with acute respiratory distress syndrome at the severe end of the disease. Facts recommended the occurrence of rupture of the basement membranes and interstitial matrix remodeling during ALI. Matrix metalloproteinases (MMPs) participate in tissue remodeling related with pathological conditions such as ALI. We hypothesized the interrelationships between extracellular matrix (ECM) turnover as MMP-9 and indicator of angiogenesis such as angiopoietin-2 (Ang-2) as well as plasma von Willebrand factor (vWF) and their correlation with arterial partial pressure of oxygen $\left(\mathrm{PaO}_{2}\right)$, oxygen saturation $\left(\mathrm{SaO}_{2}\right)$ and mortality in ALI/ARDS.

Methods: Eighty-eight mechanically ventilated patients (68 male, mean (SD) age 61 (10) years) were compared with 40 healthy controls (36 male, mean (SD) age $57(10)$ ). All biomarkers were measured by ELISA. Oxygenation, body temperature, leucocytes, and platelet counts were noted.

Results: Plasma levels of all biomarkers were significantly different among ALI/ARDS subjects $(P<0.001)$ and they inversely related to $\mathrm{PaO}_{2}$ and $\mathrm{SaO}_{2}$ and positively related to mortality. Plasma levels of MMP-9 were negatively correlated with $\mathrm{PaO}_{2}$ and $\mathrm{SaO}_{2} \%$ in ALI/ARDS patients ( $r=-0.75, P<0.0001$ and $r=-0.81, P<0.0001)$ respectively. Plasma level of Ang-2 was negatively correlated with $\mathrm{PaO}_{2}$ and $\mathrm{SaO}_{2} \%$ in ALI/ARDS patients $(r=-0.68, P<0.0001$ and $r=-0.63, P<0.0001)$ respectively. Plasma levels of vWF were negatively correlated with $\mathrm{PaO}_{2}$ and $\mathrm{SaO}_{2} \%$ in ALI/ARDS patients $(r=-0.76, P<0.0001$ and $r=-0.69, P<0.001)$ respectively. Elevated plasma levels of all indices were interrelated at the first day of admission.

Conclusion: The observed diversity in plasma levels of MMP-9, Ang-2 and VWF in ALI/ARDS patients (Table 1) revealed the activity and severity of the disease, shedding more light on the pathogenesis and/or presentation of ARDS.

\section{() Biomed Central}


Table 1(abstract P2) Plasma levels of all biomarkers in cohort study on admission (first day of admission)

\begin{tabular}{llll}
\hline Mean $(\mathrm{SD})$ & Healthy controls $(\boldsymbol{n}=\mathbf{4 0})$ & ALI/ARDS patients $(\boldsymbol{n}=\mathbf{8 8})$ & $\boldsymbol{P}$ value \\
\hline MMP-9 $(\mathrm{ng} / \mathrm{ml})$ & ${ }^{\mathrm{a}} 90.23(17.94)$ & $582.00(87.36)$ & $<0.001$ \\
Ang-2 $(\mathrm{ng} / \mathrm{ml})$ & ${ }^{\mathrm{a}} 12.48(3.26)$ & $297.30(52.51)$ & $<0.001$ \\
VWF $(\mathrm{U} / \mathrm{ml})$ & ${ }^{\mathrm{a}} 124.50(38.69)$ & $367.40(64.39)$ & $<0.001$ \\
\hline
\end{tabular}

Data presented as mean (SD). MMP-9, matrix metalloproteinase-9; Ang-2, angiopiotein-2; vWF, von Willebrand factor. $P$ value by Mann-Witney test. ALI/ARDS patients were significantly different compared with healthy controls. ${ }^{\mathrm{a}} P<0.001$.

P3

Thalidomide modulates macrophage-mediated inflammatory innate immune response during Klebsiella pneumoniae B5055 infection in BALB/c mice

$\checkmark$ Kumar, S Chhibber

Panjab University, Chandigarh, India

Critical Care 2012, 16(Suppl 3):P3

Background: Lung innate immune response plays an important role in the clearance of pathogens (that is, bacteria, virus or fungi) from lungs. However, profound activation of innate immune cells (alveolar macrophages or neutrophils) can lead to development of acute lung inflammation or injury observed during pneumonia, acute respiratory distress syndrome (ARDS), or sepsis by producing various proinflammatory cytokines (that is, IL-1 $\alpha$, TNF $\alpha$ ) and molecules (that is, hydrogen peroxide $\left.\left(\mathrm{H}_{2} \mathrm{O}_{2}\right)\right)$. Thalidomide is a drug, which has its own place in the history of medicine and has been used in various types of cancers and other chronic inflammatory disorders (erythema nodosum leprosum) but its mode of action as an immunomodulatory drug in acute infections (that is, pneumonia and sepsis) is not clear. Thus, the present study was designed to investigate its effect on pulmonary innate immune response during acute lung infection in BALB/c mice.

Methods: Animals were divided into four groups ( $n=20$ for each group). Acute lung inflammation was induced by intranasal instillation of Klebsiella pneumoniae B5055 into mice without any anesthesia and treated with thalidomide (30 mg/kg/day/p.o.) or normal saline orally using a treatment schedule shown to modulate proinflammatory innate immune response. Various proinflammatory (IL-1 $\alpha, \mathrm{TNF} \alpha$ ) as well as anti-inflammatory (that is, IL-10) cytokines were estimated by ELISA. Pulmonary macrophagemediated phagocytosis and bactericidal assays were performed. Alveolar macrophages were evaluated in terms of macrophage spreading ability assay. $\mathrm{H}_{2} \mathrm{O}_{2}$ production by macrophages was assessed according to the method based on the process of horseradish peroxidase-dependent oxidation of phenol red assay. Neutrophil infiltration to the lungs was determined by histopathological analysis.

Results: Thalidomide treatment modulated proinflammatory function of alveolar macrophages by significantly decreasing their phagocytic potential in terms of phagocytic uptake and intracellular killing, spreading and $\mathrm{H}_{2} \mathrm{O}_{2}$ release. Besides that, thalidomide treatment also significantly decreased neutrophil infiltration into the lung alveoli. Remarkably, the levels of proinflammatory cytokines (IL-1 $\alpha$ and TNF $\alpha$ ) were found to be decreased significantly in the thalidomide-treated group but the levels of IL-10 were found to be significantly elevated.

Conclusion: Thalidomide proved a promising immunomodulatory agent with a potential to modulate aggravated innate immune response observed during acute lung inflammation associated with pneumonia or sepsis caused by Gram-negative bacterial infection.
P4.

Immunoglobulin therapy of abdominal sepsis in emergency surgery

O Butyrsky*, V Starosek

Crimean Medical University, Simferopol, Ukraine

Critical Care 2012, 16(Suppl 3):P4

Background: The literature data demonstrate that the initial stage of abdominal sepsis (AS) is characterized with typical immune shifts as white blood cell (WBC) activation, decrease of T-lymphocyte and B-lymphocyte function followed by dysimmunoglobulinemia. As AS progresses immunity deficiency acquires severe combined character. Impact of immunity deficiency on a course is not described.

Methods: We investigated 33 patients (male:female $=25: 8$ ) with abdominal sepsis (total peritonitis following appendicitis, perforated duodenal ulcer, pancreonecrosis). Anti-endotoxin (AE) antibodies (antiLPS-IgA, anti-LPS-IgM, anti-LPS-IgG) were determined by original modification of hard-phase immunoenzyme analysis. Escherichia coli K30 LPS was used as antigen for AE antibody detection. All data were compared with healthy donors (99 patients). As a method of immunity correction we selected Sandoglobulin (Novartis, Switzerland), introduced as $3 \mathrm{ml}$ once intravenously.

Results: According to results of immunity parameter investigation on the day of admission we shared all patients into two groups: with high initial immunity ( $n=6$, no evidence from normal parameters) and low initial immunity ( $n=27$, evident decrease of AE immunity parameters). All patients needed comprehensive medication support. The patients with high immunity did not need any immunotherapy. But the patients with low initial immunity required immune therapy to avoid severe course of peritonitis and unfavorable outcomes. Single introduction of Sandoglobulin on the fifth day after surgery was accompanied with increase of immunity indices (Table 1). Simultaneously with an increase of anti-LPS-immunoglobulin titer one noticed sharp positive clinical changes (improvement of patients' self-feeling, body temperature decrease, decrease/normalization of WBC number, neutrophil shift). We have the following data about routine laboratory parameters in abdominal sepsis patients with Sandoglobulin introduction: WBC, $\times 10^{9} /$ l, $16.5 \pm 0.9$ before introducing and $9.8 \pm 1.2$ in 2 days after introducing; stab neutrophils, $\%, 18.3 \pm 1.6$ and $10.8 \pm 1.4$ appropriately juvenile neutrophils, \%, $4.1 \pm 0.8$ and $0.3 \pm 0.1$ appropriately (all changes are evidently proved, $P<0.001)$. Successful surgical treatment and immunotherapy of abdominal sepsis are accompanied by activation of $A E$ immunity, all-class anti-LPS-immunoglobulin concentration growth that blocks mechanisms of further inflammation progress and result in rapid patients' recovery.

Conclusion: Peritonitis patients with initial low immunity need passive nonspecific immunotherapy that stimulates organism protective functions and promotes rapid recovery.

Table 1(abstract P4) Dynamics of AE antibodies in abdominal sepsis patients with Sandoglobulin introduction

\begin{tabular}{lll}
\hline & Patients with low immunity level $(\boldsymbol{n}=\mathbf{2 7})$ & \\
\cline { 2 - 3 } & Before introduction (optic units) & 2 days after introduction (optic units) \\
\hline Anti-LPS-IgA & $0.154 \pm 0.015$ & $0.342 \pm 0.02^{*}$ \\
Anti-LPS-IgM & $0.213 \pm 0.01$ & $0.284 \pm 0.02^{*}$ \\
Anti-LPS-lgG & $0.083 \pm 0.007$ & $0.186 \pm 0.04^{*}$ \\
\hline
\end{tabular}

${ }^{*} P<0.001$. 
P5

Procalcitonin level as a marker of severe sepsis and septic shock patients who required polymyxin-B immobilized fiber with direct hemoperfusion

T Ikeda*, K Ikeda, S Suda

Tokyo Medical University Hachioji Medical Center, Tokyo, Japan

Critical Care 2012, 16(Suppl 3):P5

Background: When septic patients progress to endotoxin shock, the mortality rate becomes high and is within 30 to $80 \%$ in those with multiple organ failure.

Methods: We evaluated the levels of procalcitonin (PCT) and markers of sepsis (IL-6, IL-8, IL-1ra and PAI-1) in 159 patients who had severe sepsis and septic shock. Before starting the polymyxin-B immobilized fiber with direct hemoperfusion (PMX-DHP) treatment, patients were divided into three groups according to their PCT levels ( $L$ group: $0.5<P C T \leqq 2.0 \mathrm{ng} / \mathrm{ml}$, $M$ group: $2.0<P C T \leqq 10.0 \mathrm{~g} / \mathrm{ml}, \mathrm{H}$ group: $\mathrm{PCT}>10.0 \mathrm{ng} / \mathrm{ml}$ ).

Results: Sixty-two percent of the patients showed high PCT levels $(>10.0 \mathrm{ng} / \mathrm{ml})$. The APACHE II score and Sequential Organ Failure Assessment score tended to be high in the $\mathrm{H}$ group, but there was no significant difference among the groups. The survival rate declined with high PCT levels. The levels of inflammatory (IL-6 and IL-8) and antiinflammatory (IL-1 ra) cytokines tended to be high in the $\mathrm{H}$ group, but there was no statistically significant difference among the groups. On the other hand, the PAI-1 level was significantly increased in the $\mathrm{H}$ group (498 $\pm 499 \mathrm{ng} / \mathrm{ml})$ compared with the $L$ group $(157 \pm 103 \mathrm{ng} / \mathrm{ml}$ ) and M group (311 $\pm 319 \mathrm{ng} / \mathrm{ml})$.

Conclusion: Sixty-two per cent of patients who required PMX-DHP treatment had high PCT levels. PCT might be a mediator of sepsis and sepsis markers. PCT can potentially affect sepsis-related markers.

P6

Diagnostic accuracy of procalcitonin in proven and clinically suspected systemic infection

S Das, D Anand, S Ray, S Bhargava, A Manocha, M Kankra, LM Srivastava

Sir Ganga Ram Hospital, New Delhi, India

Critical Care 2012, 16(Suppl 3):P6

Background: Sepsis is a common cause of morbidity and mortality in critically ill patients. Microbiological culture is the gold standard for diagnosis of sepsis but unfortunately culture results are positive in only 30 to $50 \%$ patients. Sepsis is also difficult to distinguish from systemic inflammatory response syndrome (SIRS) because of similar clinical presentations. Procalcitonin (PCT) and IL- 6 are known biochemical markers for diagnosis and prognosis of sepsis. The aim of this study was to evaluate the diagnostic role of PCT and IL- 6 in differentiating sepsis (both culture positive and culture negative) from SIRS.

Methods: The study comprised three patient groups, age $>18$ years: group $1(n=41)$, proven infection; group $2(n=29)$, clinically suspected infection but negative culture; group $3(n=29)$, patients with SIRS. Blood was collected at the time of admission for microbiological culture and estimation of PCT (TRACE, Kryptor) and IL-6 (CLIA, Access).

Results: The median PCT level was significantly higher $(P<0.001)$ in both groups 1 and 2 as compared with group 3, whereas the median IL-6 level was significantly high $(P<0.001)$ only in group 1 as compared with group 3. Receiver operating characteristic (ROC) curve analysis between groups 1 and 3 for prediction of systemic infection demonstrates that both PCT and IL- 6 have a significant area under the curve (AUC) of 0.923 $(P<0.001)$ and $0.824(P<0.001)$ respectively. The best cutoff point of PCT was at $1.48 \mathrm{ng} / \mathrm{ml}$ with $85 \%$ sensitivity, $83 \%$ specificity, $88 \%$ positive predictive value (PPV) and $80 \%$ negative predictive value (NPV). However, the best cutoff point for IL-6 was very high at $219 \mathrm{pg} / \mathrm{ml}$, with $78 \%$ sensitivity, $76 \%$ specificity, $82 \%$ PPV and $71 \%$ NPV. Similarly, ROC curve analysis between groups 2 and 3 demonstrates that PCT has a significant AUC of $0.848(P=0.001)$, whereas the AUC of IL- 6 is 0.555 , which is not significant $(P=0.47)$. The best cutoff point for PCT was at $1.45 \mathrm{ng} / \mathrm{ml}$ with $83 \%$ sensitivity, $79 \%$ specificity, $80 \%$ PPV and $82 \%$ NPV, whereas the best cutoff point of IL- 6 was $98.25 \mathrm{pg} / \mathrm{ml}$, with only $59 \%$ sensitivity, $55 \%$ specificity, 57\% PPV and 57\% NPV.
Conclusion: In differentiating SIRS from sepsis, IL- 6 does not have a diagnostic role in culture negative sepsis patients, whereas PCT showed a better accuracy in differentiating SIRS from both proven and suspected sepsis. Hence, inclusion of PCT in the initial diagnostic strategy may aid in early and appropriate therapeutic intervention in culture-negative sepsis patients.

P7

Evaluation of a soluble CD14 subtype in patients with surgical sepsis

Y Fukui

Kochi Health Sciences Center, Kochi, Japan

Critical Care 2012, 16(Suppl 3):P7

Background: Surgical sepsis remains a cause of poor outcome. In order to treat surgical sepsis appropriately, it is important to reach diagnosis of sepsis early during the course and start antibiotic treatment. The gold standard of sepsis diagnosis is blood culture but there are issues with its sensitivity along with contamination. Soluble CD14 subtype (sCD14st) is an $\mathrm{N}$-terminal fragment of CD14, and signal messenger of lipopolysaccharide (LPS). sCD14st is reported to increase in sepsis patients. In this study, we evaluated the specificity, clinical effectiveness of soluble sCD14st in patients with surgical sepsis.

Methods: The study population was 18 operated patients in Kochi Health Sciences Center between April 2010 and March 2011. We investigated sCD14st using the compact automated enzyme immunoanalyzer PATHFAST (Mitsubishi Chemical Medience Co., Japan) and other biomarkers (procalcitonin (PCT), CRP, IL-6) at surgery (day 0) followed by 1, 3, 5, 7 days. APACHE II and Sequential Organ Failure Assessment (SOFA) were used to assess the sepsis severity. Sepsis was diagnosed according to the American College of Chest Physicians/Society of Critical Care Medicine (ACCP/SCCM) criteria.

Results: There were nine patients with acute abdomen, esophageal cancer in five patients, cerebral bleeding in two patients, and a patient with Fournier's gangrene and trauma, respectively. The area under the curves (AUC) of receiver operating characteristic (ROC) curve analysis for septic infection were 0.92 for sCD14st, 0.82 for PCT, 074 for IL-6, and 0.82 for CRP. Two esophageal cancer patients who indicated over $800 \mathrm{pg} / \mathrm{ml}$ in sCD14st had complicated nosocomial pneumonia. sCD14st also positively correlated with SOFA sore $(r=0.41)$ and APACHE II score $(r=0.28)$.

Conclusion: sCD14st was a useful biomarker for the diagnosis of surgical sepsis and reflected clinical severity after operation.

P8

Advance directives and end-of-life decision-making in the ICU: results from an observational study

CS Hartog ${ }^{1,2^{*}}$, I Peschel ${ }^{1}$, D Schwarzkopf ${ }^{2}$, B Kabisch ${ }^{1}$, A Günther ${ }^{1}$, R Pfeifer ${ }^{1}$,

H Skupin ${ }^{1,2}$, K Reinhart ${ }^{1,2}$, N Riedemann

'University Hospital Jena, Friedrich-Schiller University, Jena, Germany;

${ }^{2}$ Center for Sepsis Control and Care, Integrated Research and Treatment

Center, University Hospital Jena, Jena, Germany

Critical Care 2012, 16(Suppl 3):P8

Background: Advance directives (AD) are intended to ensure patient autonomy in the event of loss of decision-making capacity. According to German law, ADs are now legally binding. It is unclear whether they are helpful in the ICU [1]. The aim of this study is to compare ICU treatment with patients' written wishes.

Methods: The setting was surgical, neurological and cardiological ICUs of one German university hospital with a total of 72 beds. All patients with AD who died on the ICU between December 2010 and December 2011 were included. ADs were collected from patients' files, and their exact specifications, wishes and preferences were extracted and tabulated. Patient demographics and medical treatment was extracted from an Electronic Patient Data Management System. The time period was 48 hours preceding death. AD were defined as applicable if they described the potentially applicable clinical situation as 'final stage of a fatal disease' or 'inevitable immediate dying process'. In contrast, AD were defined as not applicable if they described other situations, including 'mental deterioration', 'breakdown of vital organs' or '(in)direct brain damage'. 
Results: Sixty-four ICU patients were included. AD were written a median 109 weeks (IQR 26.95, 261.11 weeks) before the ICU stay. Forty-four AD were applicable according to the definition. Of 44 patients with applicable ADs, $50 \%$ were surgical patients, $41 \%$ patients had severe sepsis. Within 48 hours prior to death, $27 \%$ received a DNR/I (do-not-resuscitate/intubate) order or DND (do-not-dialyze) order. Eighteen per cent received a decision to withhold, $43 \%$ a decision to withdraw therapy. Twenty-two patients $(50 \%)$ refused intensive care, 98\% (43/44) specifically refused life-sustaining measures and, in addition, many specifically named measures to be avoided. Patients who refused life-sustaining measures received the following treatments: within 48 hours prior to death, $12 \%$ received CPR and $23 \%$ underwent surgery. Within 24 hours, $77 \%$ were mechanically ventilated and on vasopressors. At the time of death, $72 \%$ were intubated $74 \%$ with $\mathrm{FiO}_{2}>21 \%, 30 \%$ on vasopressors. Of these 43 patients, 21 were surgical and 22 nonsurgical patients. Fourteen out of 43 applicable $A D$ were pre-printed forms, 29 were written individually. No trend was noticeable regarding compliance with single patients' wishes in these subgroups.

Conclusion: $A D$ do not seem to direct end-of-life care in the ICU. Our findings strengthen concerns that $A D$ in their current form may not be suitable for this patient population. Moreover, inability to comply with patients' wishes may add to the emotional burden of ICU staff.

Reference

1. Tillyard AR: Ethics review: 'Living wills' and intensive care - an overview of the American experience. Crit Care 2007, 11:219-224.

P9

Protective effects of FCGR2A polymorphism in invasive pneumococcal diseases

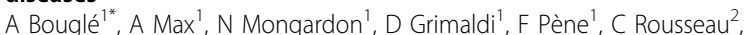
JD Chiche', JP Bedos ${ }^{3}$, E Vicaut ${ }^{4}$,JP Mira

'Hôpital Cochin, Paris, France; 'Institut Cochin, INSERM U1016/CNRS UMR8104, Paris, France; 'Hôpital André Mignot, Le Chesnay, France;

${ }^{4}$ Hôpital Lariboisière, Paris, France

Critical Care 2012, 16(Suppl 3):P9

Background: Streptococcus pneumoniae is a major cause of pneumonia and meningitis. Several genetic polymorphisms have been described to explain differences in susceptibility and severity of encapsulated pathogen-related diseases. Among them, a functional FCGR2A polymorphism leading to amino acid change of histidine $(H)$ to arginine $(R)$ at position 131 appears to be a major candidate in adult invasive pneumococcal diseases (IPD). However, previous reports needed confirmation in a large well-defined population. Methods: A prospective genetic association study in a 24-bed medical ICU of a tertiary teaching hospital over 7 years. Patients were retrospectively selected from two prospective cohorts generated between January 2001 and December 2008. All Caucasian subjects with proven IPD and hospitalized in the ICU were included. Cases of IPD were defined by the isolation of S. pneumoniae from a normally sterile site (blood, bronchoalveolar lavage,

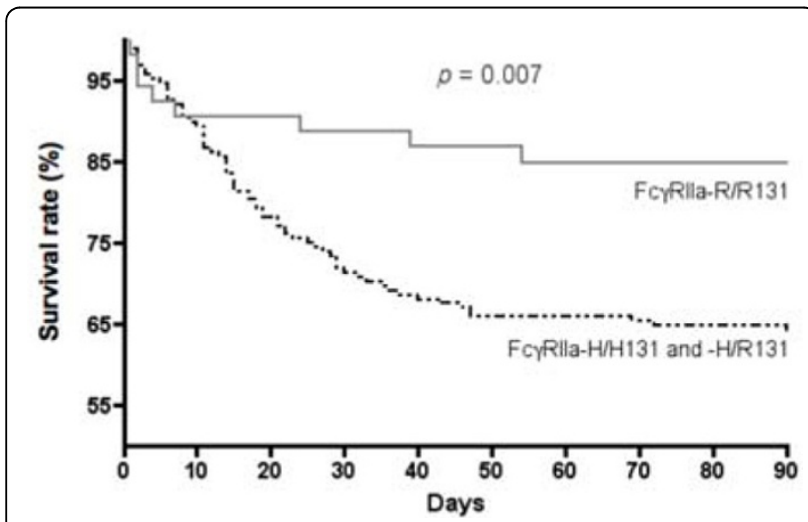

Figure 1(abstract P9) Kaplan-Meier anlaysis of the probability of survival according to FcyRlla polymorphism quantitative tracheal aspiration, cerebrospinal fluid (CSF)). DNA from all Caucasian patients with IPD (pneumonia and/or meningitis) was genotyped for the FcrRlla-R/H131polymorphism.

Results: Two hundred and forty-three patients with proven IPD were enrolled, 202 (82\%) with pneumonia and 55 (22\%) with meningitis. Mean age was 61 years, mean SAPS2 was 50.4, one-half of the patients had bacteremia, $84 \%$ of the cohort was mechanically ventilated and the hospital mortality rate was $31 \%$. In the IPD group, distribution of the Fc $\gamma \mathrm{Rlla}-\mathrm{R} / \mathrm{H} 131$ genotypes (H/H: 25\%; H/R: 53\%; RR: 22\%) was comparable with the distribution in the Caucasian control group. Comparison of the FcyRlla-R/R131 and the (FcyRlla$\mathrm{R} / \mathrm{H} 131+\mathrm{Fc \gamma Rlla}-\mathrm{H} / \mathrm{H} 131$ ) groups did not demonstrate any difference for age, SAPS2, origin of sepsis and other co-morbid conditions. However, the variant FcyRlla-R/R131 genotype was independently associated with decreased hospital mortality ( $\mathrm{OR}=0.251, \mathrm{Cl} 0.098$ to $0.645 ; P=0.004)$. See Figure 1 .

Conclusion: In a well-defined population of IPD patients, the frequency of the variant FcyRlla-R131 does not differ from other critically ill patients. However, the FcyRlla-R/R131 genotype was independently associated with increased survival regardless of the site of infection.

P10

Examination of blood filtration membrane removal ability of HMGB1

H Imahase*, Y Sakamoto, M Kusunose, H Koami, Y Nishimura, A Goto,

T Yamashita, A Nakashima, T Iwamura, N Kutsukata

Saga University, Saga City, Japan

Critical Care 2012, 16(Suppl 3):P10

Background: When the invasion of sepsis or trauma is applied to the organism, systemic inflammation occurs. In the cascade from invasion to inflammation, as one of the chemical mediators of systemic inflammation, the relationship of HMGB1 to the onset of AKI and ARDS has been pointed out. In this study we investigated the ability to remove HMGB1 by the hemofiltration membrane in vitro.

Methods: We dissolved HMGB1 in bovine serum, using a blood filtration membrane filter made of two types of membrane: heparin graft AN69ST (oXiris) and a membrane filter made of polyarylether sulfone (HF set). Filtration experiments were performed in vitro. The HMGB1 concentrations of the serum of inlet and outlet of the filter and of the filtrate were measured over time up to 120 minutes. Using an electron microscope, HMGB1 adsorption on the membrane was taken.

Results: The HMGB1 concentration $(\mathrm{ng} / \mathrm{ml})$ immediately after and 15 minutes after the start was oXiris: $15.4 \pm 1.9$ and $8.6 \pm 1.2$, HF set: $16.0 \pm 0.8$ and $16.5 \pm 1.1$ (mean \pm SD). The concentration was significantly decreased in 15 minutes immediately after the start of the oXiris set. The blood clearance ( $\mathrm{ml} /$ minute) immediately after the start was oXiris: $44.6 \pm 2.51$, HF set: $7.77 \pm 2.35$. The filtrate clearance was oXiris: $0.13 \pm 0.23$, HF set: $0.43 \pm 0.21$. In the electron microscope, HMGB1 was adsorbed on the membrane.

Conclusion: oXiris removed HMGB1 in a short time; its mechanism has been shown to be adsorption. HMGB1 can be removed from the blood, and there is a possibility to control the excessive inflammatory response during severe invasion to an organism in vivo.

P11

Decreased expression of HLA-DR antigen-associated invariant chain mRNA predicts mortality after septic shock

M-A Cazalis ${ }^{*}$, L Cavé $^{1}$, J Demaret ${ }^{2}, V$ Barbalat ${ }^{1}$, E Cerrato $^{1}$, A Lepape $^{1,3}$ A Pachot ${ }^{1}, G$ Monneret ${ }^{1,2}$, F Venet $^{1,2}$

'Joint Unit 'Sepsis', Hospices Civils de Lyon - bioMérieux, Hôpital Edouard Herriot, Lyon, France; ${ }^{2}$ Immunology Laboratory, Hôpital Edouard Herriot, Lyon, France; ${ }^{3}$ Intensive Care Units, Centre Hospitalier Lyon-Sud, Pierre Bénite, France

Critical Care 2012, 16(Suppl 3):P11

Background: Septic syndromes remain the leading cause of mortality in ICUs. Patients rapidly develop immune dysfunctions of which intensity and duration have been linked with increased risk of secondary ICU-acquired infections and death. A decreased expression on circulating monocytes of human leukocyte-antigen DR (mHLA-DR) measured by flow cytometry has been shown to be a good marker of sepsis-induced immune dysfunctions [1] and to correlate with increased risk of death and ICU-acquired 


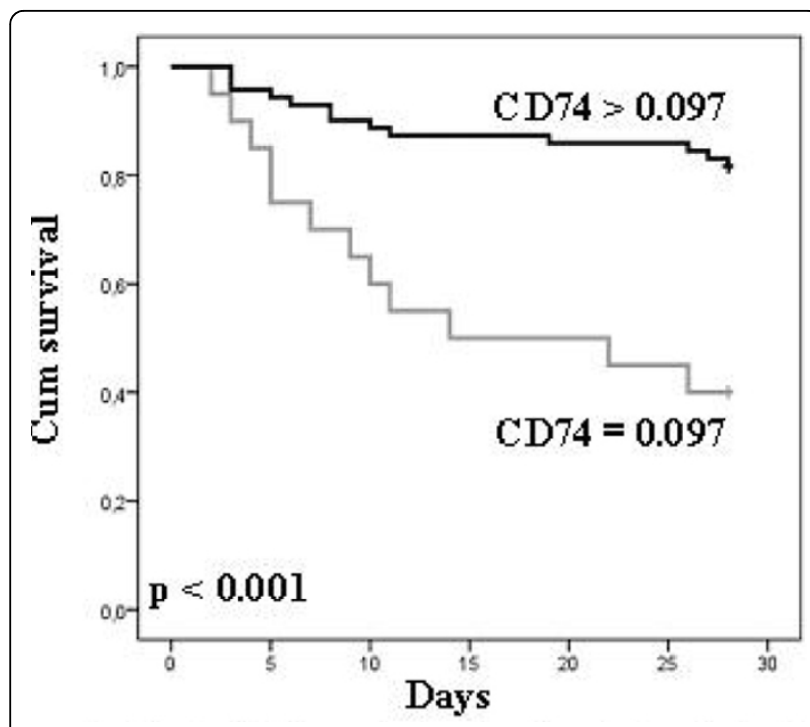

Figure 1(abstract P11) Kaplan-Meier analysis of septic shock patients' 28-day survival after stratification on CD74 mRNA

expression. The threshold was chosen based on the Youden index

calculated on the receiver operating characteristic curve. There is a significant difference between the two curves (log-rank test, $P<0.001$; hazard ratio $=7.065,95 \% \mathrm{Cl}=2.56$ to 19.48$)$.

infections [2,3]. However, pre-analytical and analytical issues inherent to mHLA-DR measurement by flow cytometry limit the use of this marker in large multicentric clinical studies and on a routine basis. We investigated whether the whole blood mRNA expression of genes related to major histocompatibility class II (MHC class II) antigens could correlate with mHLA-DR protein expression measured by flow cytometry and predict mortality in septic shock patients.

Methods: Ninety-three septic shock patients were included. PAXgene ${ }^{\otimes}$ tubes were collected at days 3 to 4 after the onset of shock. The mRNA expression of five MHC class II-related genes (that is, CD74, HLA-DRA, HLA-DMB, HLA-DMA and CIITA) was measured by qRT-PCR. In parallel, ethylene diamine tetraacetic acid-anticoagulated blood samples were collected to measure mHLA-DR expression by flow cytometry.

Results: A significant correlation $(r>0.8)$ was found between the mRNA expression of MHC class II related genes. As observed for mHLA-DR, the mRNA levels were significantly decreased in nonsurvivors as compared with survivors. The best predictive value was obtained for the invariant chain (CD74) mRNA level $(P=0.003)$. This was confirmed by the receiver operating characteristic curve analysis ( $\mathrm{AUC}=0.7,95 \% \mathrm{Cl}=0.574$ to $0.822 ; P=0.003$ ). Importantly, this remained significant after multivariate analysis including usual confounders (severity scores) and mHLA-DR values $(P=0.016)$. Survival curves showed that the decreased CD74 mRNA level was associated with increased mortality after septic shock (Figure 1).

Conclusion: Decreased invariant chain mRNA expression significantly predicts 28-day mortality in septic shock patients. After validation in a larger multicentric study, this biomarker could become a robust predictor of mortality in septic patients. This is all the more because the availability in routine laboratories of molecular biology platforms will enable standardized and routine use of such biomarkers.

\section{References}

1. Monneret G, Venet F, Pachot A, Lepape A: Monitoring immune dysfunctions in the septic patient: a new skin for the old ceremony. Mol Med 2008, 14:64-78.

2. Monneret G, Lepape A, Voirin N, Bohé J, Venet F, Debard AL, Thizy H, Bienvenu J, Gueyffier F, Vanhems P: Persisting low monocyte human leukocyte antigen-DR expression predicts mortality in septic shock. Intensive Care Med 2006, 32:1175-1183.

3. Landelle $C$, Lepape $A$, Voirin $N$, Tognet $E$, Venet $F$, Bohé J, Vanhems $P$, Monneret G: Low monocyte human leukocyte antigen-DR is independently associated with nosocomial infections after septic shock. Intensive Care Med 2010, 36:1859-1866.

P12

Kaiser Permanente Northern California sepsis mortality reduction initiative

B Crawford", M Skeath, A Whippy

Kaiser Permanente, Oakland, CA, USA

Critical Care 2012, 16(Suppl 3):P12

Background: In 2008, Kaiser Permanente Northern California (KPNC), which provides care to 3.3 million members in 21 hospitals, implemented an initiative to improve sepsis care, a critical step to reduce hospital mortality. The goals of the program were threefold: improve identification of sepsis patients, appropriately stratify risk, and reliably provide treatment, focusing on spread and sustainability across all medical centers.

Methods: In spring 2008, all hospitals reviewed the last 50 deaths and sepsis was identified as a significant improvement opportunity. In May 2008, two hospitals began rapid cycle pilot testing, resulting in the development of a playbook containing treatment algorithms, standardized order sets and flow charts, and chart abstraction tools. These tools, along with expectations for implementation, were shared with leaders and champions from all 21 hospitals at the November 2008 Sepsis Summit. The summit closed with a young mother sharing the story of how her life was saved as a result of the work at the pilot hospital. Subsequently, all hospitals convened multidisciplinary sepsis teams and began training and tool adoption, focusing immediately on improving sepsis identification. Regional mentors and medical center improvement advisors supported team-building and rapid implementation; timely and actionable data allowed ongoing identification of improvement opportunities. Identification and performance monitoring were supported by the development of a web-based tool that pulled information directly from the electronic medical record.

Results: The number of sepsis diagnoses per 1,000 admissions increased from a baseline of 35.7 (March 2008) to 98.3 (December 2010). For septic shock patients, bundle performance increased from 7.3\% (Q3 2009) to $55.1 \%$ (December 2010), and EGDT population mortality decreased from 29.7\% (July to August 2009) to $20.2 \%$ (Q4 2010). Overall sepsis mortality decreased from a baseline of $24.6 \%$ (March 2008) to $11.5 \%$ (December 2010); mortality rates continued to drop to below $9 \%$ in May 2012. This was associated with a $14 \%$ overall drop in raw hospital mortality. Subsequent performance improvement programs encompass care of the intermediate lactate population, pediatric patients and surgical patients. See Figure 1 and Table 1.

Conclusion: The KPNC program is unique in its rapid rate of improvement in sepsis measures, adoption of a single standard of care across an entire 21-hospital system, sustainability well beyond the rapid adoption period, and the quantification of mortality risk beyond the shock population to the intermediate sepsis population. These results demonstrate that a strong performance improvement engine can drive large-scale, sustained improvements in care within a short duration.

P13

Dynamics of lymphocyte subpopulations during Legionnaires' disease CPC de Jager ${ }^{\text {** }}$, EFA Gemen ${ }^{1}$, J de Jongh-Leuvenink', IBB Walsh', RJF Laheij ${ }^{1}$ $T$ van der Poll', PC Wever'

${ }^{1}$ Jeroen Bosch Hospital, ś-Hertogenbosch, the Netherlands; ${ }^{2}$ Academic

Medical Center, Center of Infection and Immunity Amsterdam and Center of Experimental and Molecular Medicine, Amsterdam, the Netherlands Critical Care 2012, 16(Suppl 3):P13

Background: Absolute lymphocytopenia (lymphocyte count $<1.0 \times 10^{9} /$ l) is recognized as an important hallmark of the immune response to severe infection and observed in patients with Legionnaires' disease (LD). Furthermore, LD is characterized by accumulation of activated T cells in the lungs. To explore the immune response in patients with LD, we studied the dynamics of peripheral blood lymphocyte subpopulations in the acute and subacute phase of the disease.

Methods: EDTA-anticoagulated blood was obtained from eight LD patients on the day the diagnosis was made (acute phase) through detection of Legionella pneumophila serogroup 1 antigen in urine. A second blood 


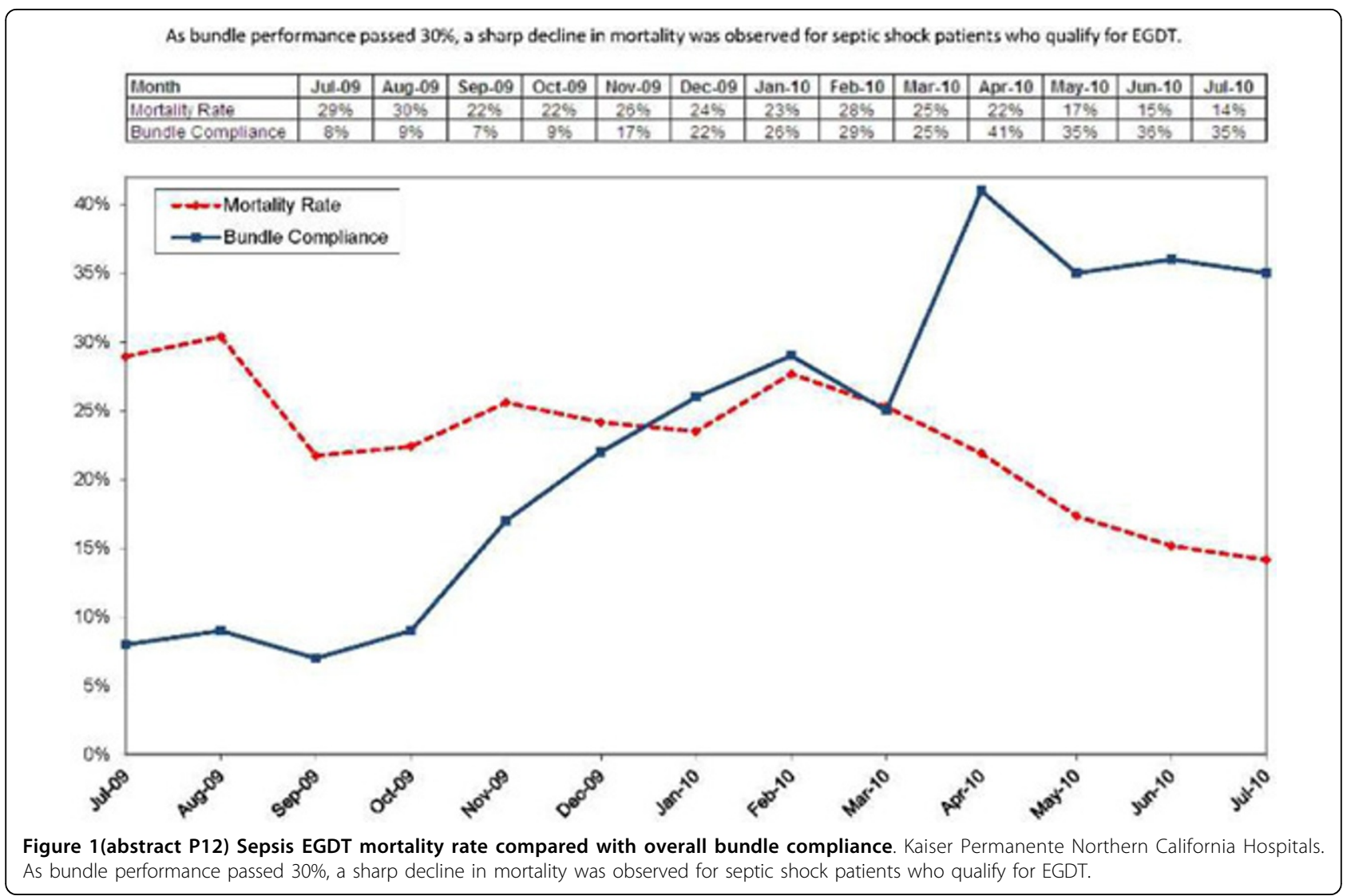

Table 1(abstract P12) Key results of the KPNC sepsis mortality reduction initiative (aggregate data for all 21 hospitals)

\begin{tabular}{|c|c|c|c|}
\hline Measure & Baseline & $\begin{array}{l}\text { Rapid adoption } \\
\text { (December 2010) }^{\text {a }}\end{array}$ & $\begin{array}{l}\text { Sustainability } \\
\text { (May 2012) }^{\mathrm{a}}\end{array}$ \\
\hline Sepsis diagnoses per 1,000 admits & 35.7 (2006 to early 2008) & 98.3 & 137.9 \\
\hline $\begin{array}{l}\text { Admitted patients with blood cultures in ED } \\
\text { have lactate test in ED }\end{array}$ & 27\% (early 2008) & $96.5 \%$ & $95.6 \%$ \\
\hline$A B X w / i n 60$ minutes of $d x$ of shock & 69.5\% (Q3 2009) & $90.4 \%$ & $91.8 \%$ \\
\hline $\begin{array}{l}\mathrm{CL} \text { w/in } 2 \text { hours of } \mathrm{dx} \text { (first CVP of ScvO2 in } \\
2 \text { hours) }\end{array}$ & 41.5\% (Q3 2009) & $78.6 \%$ & $89.6 \%$ \\
\hline Mean BP (MAP) at target & 52\% (Q3 2009) & $90.4 \%$ & $93.8 \%$ \\
\hline CVP at target & 41.5\% (Q3 2009) & $83.8 \%$ & $92.8 \%$ \\
\hline $\mathrm{ScvO}_{2}$ at target & 30.8\% (Q3 2009) & $74.3 \%$ & $81.4 \%$ \\
\hline Lactate lower within 6 hours for EGDT & 52\% (Q3 2009) & $91.2 \%$ & $95.9 \%$ \\
\hline EGDT bundle & 7.3\% (Q3 2009) & $55.1 \%$ & $70.1 \%$ \\
\hline Sepsis raw mortality & 24.6\% (2006 to early 2008) & $11.5 \%$ & $8.7 \%$ \\
\hline Sepsis observed/expected (O/E) mortality & 1.07 (rolling year ending Q1 2008) & 0.82 & 0.56 \\
\hline Sepsis O/E LOS & 1.07 (rolling year ending Q1 2008) & 0.89 & 0.75 \\
\hline $\begin{array}{l}\text { EGDT population mortality (only patients } \\
\text { with refractory shock or lactate } \geq 4 \text { ) }\end{array}$ & $\begin{array}{l}29.7 \% \text { ( } 239 \text { cases, July to } \\
\text { August 2009) }\end{array}$ & $\begin{array}{l}\text { 20.2\% (391 cases, Q4 } \\
\text { 2010) }\end{array}$ & $\begin{array}{l}\text { 18.6\% (323 cases, March-May } \\
\text { 2012) }\end{array}$ \\
\hline $\begin{array}{l}\text { Raw all cause adult non-OB KPNC hospital } \\
\text { mortality }\end{array}$ & $3.63 \%$ (2006 to 2007 ) & $3.11 \%(2010)$ & 3.07\% (year ending Q2 2012) \\
\hline HSMR-Medicate only & 0.92 (rolling year ending Q2 2008) & 0.60 (YE 2010) & 0.52 (YE 2011) \\
\hline Balancing measures: EGDT associated harm & July to December 2009 & July to December 2010 & December 2011 to May 2012 \\
\hline BSI & 0 & 0 & 0 \\
\hline Retained guidewires & 3 & 0 & 0 \\
\hline Pneumothorax & 1 & 2 & 4 \\
\hline
\end{tabular}

${ }^{a}$ Rapid adoption and sustainability data reflect December 2010 and May 2012, respectively, unless otherwise noted. 


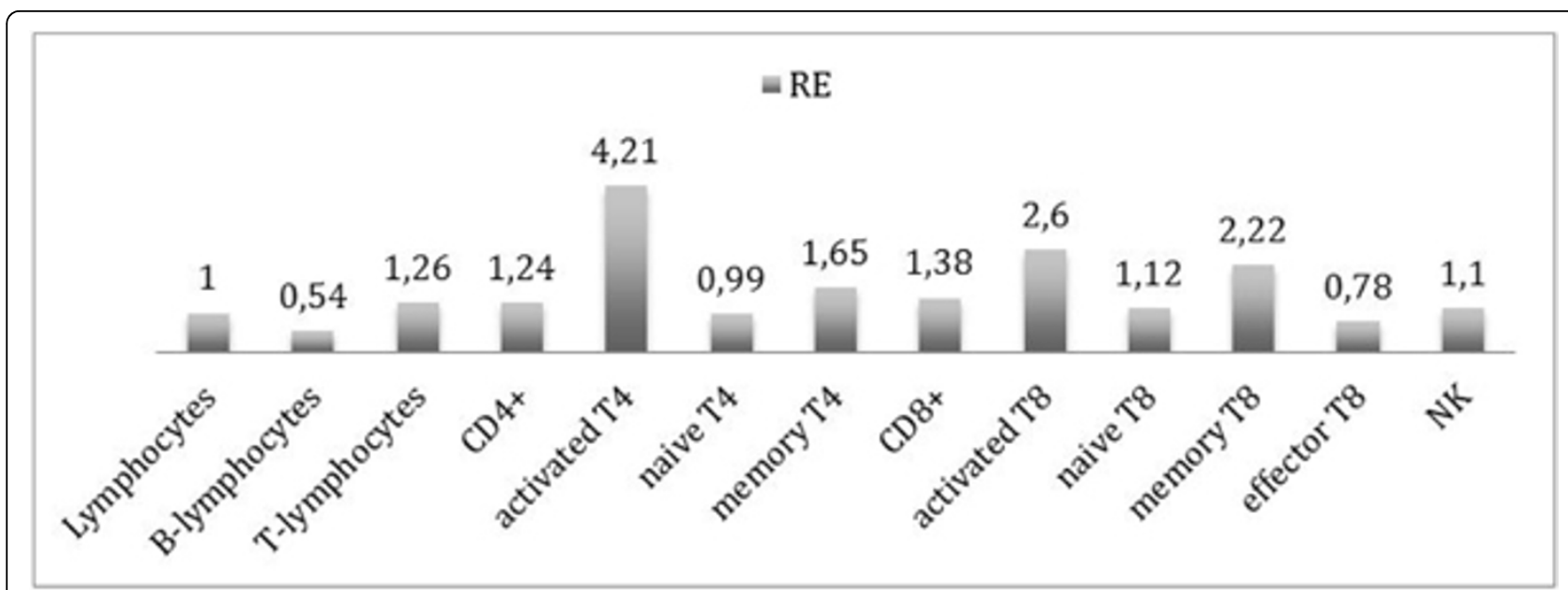

Figure 1(abstract P13) Relative expansion (mean values) of lymphocyte subsets compared with the absolute lymphocyte count in the subacute phase compared with the acute phase of Legionnaires' disease.

sample was obtained in the subacute phase. Multiparametric flow cytometry was used to calculate absolute lymphocyte counts and B-cell, T-cell, NK-cell, $\mathrm{CD}^{+}$and $\mathrm{CD} 8^{+} \mathrm{T}$-cell counts. Expression of activation markers was analyzed on $\mathrm{CD}^{+}$and $\mathrm{CD}^{+}{ }^{+}$cells. C-reactive protein (CRP) levels were used to monitor treatment response.

Results: The absolute lymphocyte count $\left(\times 10^{9} / \mathrm{l}\right.$, mean $\left.\pm \mathrm{SD}\right)$ significantly increased from $0.8 \pm 0.4$ to $1.7 \pm 0.9$ in the subacute phase. B-cell counts showed no significant change, while the T-cell count $\left(\times 10^{6} / \mathrm{l}\right)$ significantly increased in the subacute phase $(481 \pm 283$ vs. 1,290 \pm 738$)$ as a result of significant increases in both $\mathrm{CD} 4^{+}$and $\mathrm{CD} 8^{+} \mathrm{T}$-cell counts $(345 \pm 168$ vs. $898 \pm 390$ and $124 \pm 104$ vs. $333 \pm 265)$. In the $\mathrm{CD}^{+}$and $\mathrm{CD} 8^{+} \mathrm{T}$-cell populations, significant increases were observed in the subacute phase in absolute counts of activated CD38 ${ }^{+} \mathrm{HLA}^{-D R A^{+}}$cells $(11 \pm 7$ vs. $81 \pm 60$ and $14 \pm 13$ vs. $68 \pm 51)$ and CD45RA memory cells ( $141 \pm 68$ vs. $478 \pm 185$ and $28 \pm 21$ vs. $111 \pm 44$ ). Figure 1 shows the relative expansion (relative decrease/increase of a lymphocyte subset related to the relative increase of the absolute lymphocyte count) of the different lymphocyte subsets. The CRP level (mg/l) decreased from $359 \pm 72$ to $33 \pm 17$ in the subacute phase.

Conclusion: The acute phase of LD is characterized by absolute lymphocytopenia which recovers in the subacute phase with an increase in absolute T-cell count and emergence of activated and memory-type $\mathrm{CD}^{+}$and $\mathrm{CD}^{+}{ }^{+}$cells. This confirms a role for T-cell activation in the immune response to LD.

P14

Receptor for advanced glycation endproducts controls deleterious lung inflammation in severe Pseudomonas aeruginosa pneumonia in immunosuppressed mice

C Ravinet-Trillou ${ }^{1 *}$, F Soubrier ${ }^{2}$, A Caron ${ }^{2}$, S Llopart ${ }^{2}$, M Fontanie ${ }^{1}$,

F Lantreibeca ${ }^{2}$, PE Rouby' ${ }^{1}$ C Menet', L Leotard', C Serradeil-Le Gal

${ }^{1}$ Sanofi, TSU ID, Toulouse, France; '2 Sanofi, SCP Biologics, Toulouse, France

Critical Care 2012, 16(Suppl 3):P14

Background: The receptor for advanced glycation endproducts (RAGE/ AGER) plays a key role in infections by sustaining tissue-damaging inflammation. This multiligand/multisignal pattern recognition receptor, overexpressed in lung and on innate immune cells, was shown to contribute to the pathogenesis of pneumococcal pneumonia and thus may be an attractive therapeutic target to treat severe pneumonia, one of the most common causes of death in western countries. Here, we characterized a new anti-human RAGE antibody (SAR) and its in vivo effect in a model of severe Gram-negative bacterial pneumonia in immunosuppressed mice.

Methods: The SAR antibody was previously selected for binding affinity for the human sRAGE (Biacore) and for RAGE ligand binding inhibition
(S100B, HMGB1, S100A6) by ELISA. A similar $\mathrm{IC}_{50}$ was found for SAR and anti-RAGE XT-M4 antibody (Pfizer/Wyeth) with HMGB1. Since SAR did not cross-react with mouse RAGE, transgenic C57Bl6 mice expressing human RAGE were engineered for in vivo testing. Mice, receiving at day 4 and day 1 cyclophosphamide for immunosuppression, were challenged with intranasal $10^{7}$ or $10^{8}$ colony forming units (CFU) Pseudomonas aeruginosa (PA01 strain) and treated 4 hours after with ciprofloxacin alone or in the presence of the selected RAGE antibody: survival, CFU and bronchoalveolar lavage (BAL) cytokines were measured. The anti-RAGE XT-M4 antibody was used as a reference.

Results: In human RAGE KO/KI humanized mice, hRAGE expression was validated at the mRNA (RT PCR) and protein (IHC) levels. In the pneumonia model, higher loads of $P$. aeruginosa was used in transgenic versus wild-type mice to achieve more than $80 \%$ of mortality 24 hours after infection $\left(10^{8}\right.$ CFU). XT-M4 antibody was injected at $15 \mathrm{mg} / \mathrm{kg}$ intravenously 4 hours after infection (alone or with ciprofloxacin, 0.1 and $0.3 \mathrm{mg} / \mathrm{kg}$, intraperitoneal given 4 hours and 24 hours after infection). The association of XTM4 and ciprofloxacin significantly increased survival compared with the antibiotic alone (ciprofloxacin $0.1 / \mathrm{XT}-\mathrm{M} 4+54 \%, P<0.066$, ciprofloxacin $0.3 / \mathrm{XT}-\mathrm{M} 4+78 \%, P<0.01)$. The SAR antibody $(7.5$ and $15 \mathrm{mg} / \mathrm{kg})$ also induced significant improvement in delaying mortality in transgenic mice when given in combination with ciprofloxacin versus antibiotic alone. Moreover, $30 \mathrm{mg} / \mathrm{kg}$ SAR with ciprofloxacin $(10 \mathrm{mg} / \mathrm{kg}$ ) reduced (but not significantly) BAL cytokine release (TNF $\alpha, K C$, IL-12) 18 hours after infection. Conclusion: A survival benefit and partial control of inflammation was observed with anti-RAGE antibodies in combination with antimicrobial agent in a model of Gram-negative ( $P$. aeruginosa) pneumonia in immunosuppressed mice. Thus, together with existing literature reports, these data are in favor of the contribution of RAGE to the pathogenesis of severe bacterial pneumonia.

P15

Low-tidal volume ventilation as compared with conventional tidal volume ventilation in patients of sepsis: a randomized controlled trial A Waheed ${ }^{1 *}$, S Bhat $^{2}$, V Nabil' $^{1}$ S Gurcu', A Maqbool', S Patigaroo',

N Mehfooz ${ }^{3}$

${ }^{1}$ Sheri Kashmir Institute of Medical Sciences, Srinagar, India;

${ }^{2}$ Government Medical College, Srinagar, India; ${ }^{3}$ Government Medical College, Chest Diseases Hospital, Srinagar, India

Critical Care 2012, 16(Suppl 3):P15

Background: In a surgical ICU, low-tidal volume ventilation was compared with conventional tidal volume ventilation. Low-tidal volume ventilation is preferentially used to provide mechanical ventilation in support of patients with acute lung injury, acute respiratory distress syndrome, and inhalation injury. However, few prospective studies have compared low-tidal volume 
ventilation with normal tidal volume ventilation in sepsis. The purpose of this study was to prospectively compare the two ventilator modalities in an ICU setting in patients of sepsis.

Methods: A single-center, prospective, randomized controlled trial, comparing conventional tidal volume $(12 \mathrm{ml} / \mathrm{kg})$ ventilation with low-tidal volume ventilation $(6 \mathrm{ml} / \mathrm{kg})$ in patients with sepsis. In an ICU at a tertiary care teaching hospital, adult patients $\geq 18$ years of age with documented sepsis requiring prolonged ( $>24$ hours) mechanical ventilation were admitted to the ICU. Subjects were randomly assigned to receive mechanical ventilation either by conventional tidal volume ventilation ( $n=$ $40)$ or a low-tidal volume ventilation-based strategy $(n=40)$. The first primary outcome was death before a patient was shifted out of the ICU or breathing without assistance. The second primary outcome was the number of days without ventilator use from day 1 to day 28.

Results: At baseline, both the traditional ventilation group and the low-tidal volume ventilation group had similar demographics to include median age (interquartile range) ( 35 years ( 18 to 65 ) vs. 33 years (18 to 65 ), $P=$ non significant), weight (64 (50 to 82 ) versus 62 (53 to 70$)$ ). The primary outcome was ventilator-free days in the first 28 days after randomization. Our study revealed no significant difference between the low-tidal volume ventilation and the traditional strategy ventilation groups in mean $( \pm S D)$ ventilator-free days ( $12 \pm 9$ vs. $11 \pm 8, P=$ non significant) respectively), although the number of days without ventilator use during the first 28 days after randomization was greater in the low-tidal volume group. Mortality was lower in the group treated with lower tidal volumes than in the group treated with traditional tidal volumes $(27.0 \%$ vs. $30.8 \%, P=$ non significant); again the difference was not statistically significant.

Conclusion: Our study revealed that in patients with sepsis, mechanical ventilation with a lower tidal volume may be beneficial in decreasing mortality and increasing the number of ventilator-free days although the difference was found to be statistically insignificant.

P16

Impact of daily auditing and weekly feedback on process of care and patient outcome in resuscitation of severe sepsis and septic shock

B Afessa*, CB Bowron, RD Danielson, K Ramar

Mayo Clinic, Rochester, MN, USA

Critical Care 2012, 16(Suppl 3):P16

Background: Rivers' randomized clinical trial showed that early goaldirected therapy improves patient outcome in severe sepsis and septic shock [1]. The findings were confirmed by multicenter international prospective observational studies $[2,3]$. In our institution, we implemented a Quality Improvement Project based on daily auditing of patients admitted to a medical intensive care unit (MICU) for severe sepsis/septic shock followed by weekly feedback on compliance of the Institute for Healthcare Improvement (IHI) resuscitation bundle, and Sepsis Response Team (SRT) activation [4]. Once the Quality Improvement Project was completed, the daily audit and weekly feedback were stopped. This study aims to assess the impact of this change on the process of care and hospital mortality.

Methods: The study was conducted in a 24-bed adult MICU of an academic medical center. Only the first admission of each patient was included. Patients who did not authorize their medical records to be reviewed for research were excluded. During the first period of the study,
2009, daily auditing, weekly feedback to healthcare providers and SRT were implemented. During the second study period, 2010, the daily auditing and weekly feedback were stopped and the SRT activation was continued. Baseline data collected during the two periods of the study included gender, age, and resuscitation preference in case of cardiac arrest. Process of care was measured using the seven elements of the $\mathrm{IHI}$ resuscitation bundle. Patient outcome was measured by hospital mortality. Statistical comparisons between the two groups were made using Student's $t$ and chi-square tests. Two-tailed $P<0.05$ was considered statistically significant.

Results: A total of 777 patients were included in the study: 378 had auditing and feedback and 399 did not. The mean (SD) age of the feedback group was $65.4(16.77)$ years compared with $66.3(16.67)$ years for the nonfeedback group $(P=0.459)$. Female gender accounted for $147(38.9 \%)$ of the feedback group compared with $206(51.6 \%)$ of the nonfeedback group $(P<0.001)$. At MICU admission $315(83.3 \%)$ of patients in the feedback group preferred full resuscitation in case of cardiac arrest compared with $317(79.4 \%)$ in the nonfeedback group $(P=0.165)$. Compliance with all elements of the bundle declined from $50.8 \%$ during feedback to $29.8 \%$ during nonfeedback (Table 1). However, the hospital mortality rate did not increase.

Conclusion: Continuous auditing and feedback to healthcare providers is an important component of a Quality Improvement Project to maintain compliance with the severe sepsis resuscitation bundle. However, it is not associated with hospital mortality.

\section{References}

1. Rivers E, Nguyen B, Havstad S, Ressler J, Muzzin A, Knoblich B, et al: Early goal-directed therapy in the treatment of severe sepsis and septic shock. N Engl J Med 2001, 345:1368-1377.

2. Ferrer R, Artigas A, Levy MM, Blanco J, Gonzalez-Diaz G, GarnachoMontero J, et al: Improvement in process of care and outcome after a multicenter severe sepsis educational program in Spain. JAMA 2008, 299:2294-2303.

3. Levy MM, Dellinger RP, Townsend SR, Linde-Zwirble WT, Marshall JC, Bion J, et al: The Surviving Sepsis Campaign: results of an international guideline-based performance improvement program targeting severe sepsis. Crit Care Med 2010, 38:367-374

4. Schramm GE, Kashyap R, Mullon JJ, Gajic O, Afessa B: Septic shock: a multidisciplinary response team and weekly feedback to clinicians improve the process of care and mortality. Crit Care Med 2011, 39:252-258.

P17

Candida score: a predictor of mortality in patients with candidemia

D Juneja*, P Nasa, O Singh, Y Javeri, S Garg

Max Super Speciality Hospital Saket, New Delhi, India

Critical Care 2012, 16(Suppl 3):P17

Background: Candidemia is becoming increasingly widespread, especially in the ICU setup. The Candida score has been developed and used for identifying patients at risk for developing candida infections. However, its usefulness in predicting outcome of patients with candidemia has not been evaluated. We aimed to determine the risk factors for mortality in patients with candidemia admitted to an Indian medical ICU.

Table 1(abstract P16) Process of care and patient outcome differences between the two groups

\begin{tabular}{llll}
\hline Variable & Feedback $(\boldsymbol{n}=\mathbf{3 7 8})$ & No feedback $(\boldsymbol{n}=\mathbf{3 9 9})$ & $\boldsymbol{P}$ value \\
\hline Lactate measured & $369(97.6 \%)$ & $376(94.2 \%)$ & 0.018 \\
Blood culture & $364(96.3 \%)$ & $370(92.7 \%)$ & 0.030 \\
Antibiotic & $344(91.0 \%)$ & $342(85.7 \%)$ & 0.022 \\
Fluid achieved CVP & $281(74.3 \%)$ & $238(59.6 \%)$ & $<0.001$ \\
Vasopressor & $331(87.6 \%)$ & $330(82.7 \%)$ & 0.057 \\
RBC transfusion & $331(87.6 \%)$ & $336(84.2 \%)$ & 0.180 \\
Inotrope & $220(58.2 \%)$ & $146(36.6 \%)$ & $<0.001$ \\
Compliance with all & $192(50.8 \%)$ & $119(29.8 \%)$ & $<0.001$ \\
Hospital death & $92(24.3 \%)$ & $73(18.3 \%)$ & 0.040
\end{tabular}


Table 1(abstract P17) Comparison patient parameters among survivors and nonsurvivors

\begin{tabular}{lllll}
\hline Parameter of interest & Overall $(\boldsymbol{n}=\mathbf{5 6})$ & Survivors $(\boldsymbol{n}=\mathbf{3 7})$ & Nonsurvivors $(\boldsymbol{n}=\mathbf{1 9})$ & $\boldsymbol{P}$ value \\
\hline Age, mean & $63.07 \pm 20.7$ & $59.11 \pm 21.9$ & $70.79 \pm 16.1$ & $0.045^{*}$ \\
Sex, males (\%) & $41(73.2 \%)$ & $27(73 \%)$ & $14(73.7 \%)$ & 0.955 \\
APACHE II score & $19.38 \pm 7.7$ & $17.32 \pm 7.6$ & $23.37 \pm 6.2$ & $0.004^{*}$ \\
Predicted death rate (\%) & $36.87 \pm 22.6$ & $31.05 \pm 21.7$ & $48.19 \pm 20.2$ & $0.006^{*}$ \\
Nonalbicans infection & $44(78.6 \%)$ & $31(83.8 \%)$ & $13(68.4 \%)$ & 0.302 \\
Co-existing candiduria & $15(26.8 \%)$ & $10(27 \%)$ & $5(26.3 \%)$ & 0.955 \\
Underlying co-morbidities & $41(73.2 \%)$ & $24(64.9 \%)$ & $18(94.7 \%)$ & $0.021^{*}$ \\
Previous antimicrobials & $49(87.5 \%)$ & $33(89.2 \%)$ & $16(84.2 \%)$ & 0.679 \\
Previous antifungals & $8(14.3 \%)$ & $1(0.3 \%)$ & $7(36.8 \%)$ & $0.001^{*}$ \\
Days in ICU before positive culture & $12.88 \pm 14.4$ & $13.05 \pm 14.6$ & $12.53 \pm 14.3$ & 0.898 \\
Recent hospitalization & $17(30.4 \%)$ & $11(29.7 \%)$ & $6(31.6 \%)$ & 0.887 \\
Total parenteral nutrition & $18(32.1 \%)$ & $15(40.5 \%)$ & $3(15.8 \%)$ & 0.076 \\
Candida score ( $\geq 3)$ & $29(51.8 \%)$ & $15(40.5 \%)$ & $14(73.7 \%)$ & $0.025^{*}$ \\
Presence of CVC & $49(87.5 \%)$ & $31(83.8 \%)$ & $18(94.7 \%)$ & 0.403 \\
Mechanical ventilation & $36(64.3 \%)$ & $19(51.4 \%)$ & $17(89.5 \%)$ & $0.007^{*}$ \\
Vasopressor support & $30(53.6 \%)$ & $13(35.1 \%)$ & $17(89.5 \%)$ & $0.000^{*}$ \\
Renal replacement therapy & $23(41.1 \%)$ & $9(24.3 \%)$ & $14(73.7 \%)$ & $0.001^{*}$ \\
Length of ICU stay (days) & $22.86 \pm 28$ & $22.35 \pm 31.4$ & $23.84 \pm 20.6$ & 0.853 \\
Length of hospital stay (days) & $30.09 \pm 30.2$ & $33.19 \pm 33.8$ & $24.05 \pm 21.1$ & 0.288 \\
\hline
\end{tabular}

*Statistically significant.

Methods: An 18-month retrospective cohort analysis including 56 patients with candidemia was conducted. Baseline patient characteristics, ICU course and outcome were noted in a predesigned pro forma. The Candida score was calculated as previously described [1]. The primary outcome measure was ICU mortality.

Results: Out of 3,142 ICU admissions, the incidence of candidemia was $17.8 / 1,000$ admissions. Fifteen patients had co-existing candiduria with the same species. The mean interval between ICU admission and candidemia was $12.9 \pm 14.4$ days. Candida albicans was isolated in only $21.4 \%$ of blood Candida-positive cultures. Among the nonalbicans species $C$. tropicalis was the commonest species isolated from $28.6 \%$ isolates, followed by C. glabrata that was isolated from seven $(12.5 \%)$ patients. In $8.9 \%$ of candidemia patients, the only measure taken was removal of the indwelling catheter, but the rest of the patients required antifungal drugs. Among the patients with candidemia, 53.6\% required vasopressor support, $41.1 \%$ required renal replacement therapy and $64.3 \%$ required mechanical ventilation during their ICU stay. The mean length of ICU stay was $22.9 \pm$ 28 days and the mean hospital stay was $30.1 \pm 30.2$ days. Crude ICU mortality was $33.93 \%$. There was no statistically significant difference between mortality rates of patients with $C$. albicans and nonalbicans candidemia $(P=0.732)$. Patient parameters such as age, admission APACHE II score, candida score, previous antifungals and underlying comorbidities, which were statistically significant in differentiating survivors and nonsurvivors in the univariate analysis (Table 1), were included in the multivariate analysis. Only two factors, previous antifungals $(P=0.004$, $\mathrm{OR}=101.4,95 \% \mathrm{Cl}=4.52$ to 227.7$)$ and Candida score $>3(P=0.028, \mathrm{OR}=$ $13.2,95 \% \mathrm{Cl}=1.3$ to 125 ) were found to be independently predicting mortality.

Conclusion: Candida infection is generally late-onset in ICU patients and is associated with a prolonged ICU and hospital stay, and a high mortality. Candida nonalbicans infection was much more common in our cohort of ICU patients but there was no difference in mortality among patients with $C$. albicans and nonalbicans infection. Patients who develop candidemia, despite being on antifungals, were at a higher risk of dying and a simple bedside candida score may be useful in predicting mortality of ICU patients with candidemia.

Reference

1. León C, Ruiz-Santana S, Saavedra P, Almirante B, Nolla-Salas J, AlvarezLerma F, Garnacho-Montero J, León MA, EPCAN Study Group: A bedside scoring system (Candida score) for early antifungal treatment in nonneutropenic critically ill patients with Candida colonization. Crit Care Med 2006, 34:730-737.

P18

Incidence and prognostic implications of acute kidney injury based on the RIFLE criteria at the time of admission to an Indian ICU

D Juneja,, P Nasa, O Singh, Y Javeri, S Garg

Max Super Speciality Hospital Saket, New Delhi, India

Critical Care 2012, 16(Suppl 3):P18

Background: Acute kidney injury (AKI) is an important predictor of outcome in patients admitted to ICUs. The Acute Dialysis Quality Initiative (ADQI) Group has defined and stratified AKI according to the Risk, Injury, Failure, Loss and End-stage renal disease (RIFLE) criteria [1]. We aimed to assess the ability of the RIFLE criteria to predict mortality in critically ill patients admitted to a medical ICU.

Methods: A retrospective cohort study in an eight-bed medical ICU of a tertiary care hospital over a period of 16 months. Data regarding patient demographics and ICU course including need for organ support and length of stay were recorded. We classified each patient according to their RIFLE class using admission creatinine values (no AKI: $<1.5 \times$ baseline, Risk: $\geq 1.5 \times$ baseline, Injury: $\geq 2 \times$ baseline or Failure: $\geq 3 \times$ baseline), as previously described [1]. Qualitative data were analyzed using the chisquared or Fisher exact test as appropriate and quantitative data were analyzed using Student's $t$ test. Inter-group and intra-group comparison for quantitative data was done by one-way ANOVA. The primary outcome measure was the ICU mortality, which was compared in five groups of patients: no $A K I$, risk $(R)$, injury (I), failure $(F)$, and loss or end-stage (L/E).

Results: Data from 722 patients were included, no AKI: 362 (50.1\%), risk: $168(22.9 \%)$, injury: 71 (9.8\%), failure: $80(11.1 \%)$ and loss or end-stage: 44 (6.1\%). Patients were evenly matched with regards to age and sex. The ICU mortality was: no AKI $(7.5 \%)$, risk (15.8\%), injury $(25.4 \%)$, failure (38.8\%) and L/E (20.5\%). The need for renal support also varied according to RIFLE criteria: no AKI (1.1\%), risk (4.2\%), injury (26.8\%), failure $(72.5 \%)$ and L/E (77.3\%) (Table 1).

Conclusion: The RIFLE classification is a simple tool, which can be used to assess and classify AKI on admission to ICU. Moreover, it can reliably be used to predict and prognosticate outcome and need for renal support in ICU patients. 
Table 1(abstract P18) Comparison among patients according to RIFLE criteria

\begin{tabular}{llllll}
\hline Parameter of interest & $\begin{array}{l}\text { No acute kidney injury } \\
(\boldsymbol{n}=\mathbf{3 6 2})\end{array}$ & $\begin{array}{l}\text { Risk class } \\
(\boldsymbol{n}=\mathbf{1 6 5})\end{array}$ & $\begin{array}{l}\text { Injury class } \\
(\boldsymbol{n}=\mathbf{7 1})\end{array}$ & $\begin{array}{l}\text { Failure class } \\
(\boldsymbol{n}=\mathbf{8 0})\end{array}$ & $\begin{array}{l}\text { Loss or end-stage renal disease } \\
(\boldsymbol{n}=\mathbf{4 4})\end{array}$ \\
\hline Mean age (years) \pm SD & $57.89 \pm 18.2$ & $59.68 \pm 18.4$ & $57.14 \pm 16.7$ & $56.51 \pm 21.3$ & $63.09 \pm 14.7$ \\
Sex, males (\%) & $213(58.8 \%)$ & $96(58.2 \%)$ & $41(57.7 \%)$ & $41(51.3 \%)$ & $27(61.4 \%)$ \\
$\begin{array}{l}\text { Mean APACHE II score } \pm \\
\text { SD }\end{array}$ & $10.68 \pm 6.9$ & $14.64 \pm 7.6$ & $19.7 \pm 8.2$ & $24.34 \pm 8.1$ & $16.91 \pm 8$ \\
$\begin{array}{l}\text { Mean PDR } \pm \text { SD } \\
\text { Mean SOFA score } \pm \text { SD }\end{array}$ & $16.09 \pm 15.8$ & $24.33 \pm 19.2$ & $37.13 \pm 23.7$ & $49.45 \pm 23.4$ & $30.66 \pm 21$ \\
$\begin{array}{l}\text { Need for inotropic } \\
\text { support }\end{array}$ & $56(15.5 \%)$ & $5.27 \pm 3.3$ & $7.15 \pm 3.7$ & $8.95 \pm 3.4$ & $5.39 \pm 3.7$ \\
$\begin{array}{l}\text { Need for renal support } \\
\text { Need for mechanical }\end{array}$ & $4(1.1 \%)$ & $41(24.8 \%)$ & $28(39.4 \%)$ & $40(50 \%)$ & $14(31.8 \%)$ \\
ventilation & $66(18.2 \%)$ & $7(4.2 \%)$ & $19(26.8 \%)$ & $58(72.5 \%)$ & $34(77.3 \%)$ \\
$\begin{array}{l}\text { Length of ICU stay, days } \\
\text { Length of hospital stay, } \\
\text { days }\end{array}$ & $4.53 \pm 5.5 \pm 7.1$ & $43(26.1 \%)$ & $28(39.4 \%)$ & $32(40 \%)$ & $15(34.1 \%)$ \\
ICU mortality & $27(7.5 \%)$ & $5.25 \pm 5.1$ & $4.46 \pm 4.8$ & $6.44 \pm 6.5$ & $5.68 \pm 6.2$ \\
\hline
\end{tabular}

\section{Reference}

1. Bellomo R, Ronco C, Kellum JA, Mehta RL, Palevsky P, ADQI Workgroup: Acute renal failure: definition, outcome measures, animal models, fluid therapy and information technology needs: the Second International Consensus Conference of the Acute Dialysis Quality Initiative (ADQI) Group. Crit Care 2004, 8:R204-R212.

P19

Candiduria in ICUs: incidence, course and outcome

D Juneja*, P Nasa, O Singh, Y Javeri, S Garg

Max Super Speciality Hospital Saket, New Delhi, India

Critical Care 2012, 16(Suppl 3):P19

Background: There is a high incidence of candiduria especially in ICU patients. Candiduria may be a sign of a disseminated candida infection and a marker for increased ICU mortality. However, there is always a

\section{Table 1(abstract P19) Patient demographics, ICU course} and outcome

\begin{tabular}{ll}
\hline Parameter of interest & Frequency \\
\hline Age (years) ( \pm SD) & $59.81( \pm 19.5)$ \\
Sex, males (\%) & $54(58.1 \%)$ \\
Days in ICU before positive culture ( \pm SD) & $10.11( \pm 9.8)$ \\
Presence of urinary catheter (\%) & $75(80.6 \%)$ \\
Days since urinary catheter was present $( \pm$ SD) & $5.89( \pm-3)$ \\
Admission APACHE II score & $17.13( \pm 7.8)$ \\
Predicted death rate (\%) & $30.36( \pm 21.1)$ \\
Previous antimicrobials (\%) & $80(86 \%)$ \\
Recent hospitalization (\%) & $7(7.5 \%)$ \\
Neutropenia (\%) & $3(3.2 \%)$ \\
Nonalbicans infection (\%) & $62(66.7 \%)$ \\
Blood culture positivity (\%) & $18(19.4 \%)$ \\
Need for vasopressor support & $52(55.9 \%)$ \\
Need for renal replacement therapy & $25(26.9 \%)$ \\
Need for mechanical ventilation & $60(64.5 \%)$ \\
ICU length of stay (days) & $21.34( \pm 23.3)$ \\
Hospital length of stay (days) & $27.38( \pm 25.2)$ \\
ICU mortality (\%) & $27(29 \%)$ \\
\hline
\end{tabular}

dilemma regarding which patient to treat, as in a majority of patients it may only be a colonizer. Moreover, the choice of antifungal drug is also controversial due to low urinary concentration of many antifungal drugs. Hence, it becomes imperative to have knowledge of locally prevalent species to guide treatment protocols. The aim of this study was to assess the incidence of candiduria among patients admitted to a medical ICU of an Indian hospital, to perform microbiological characterization and to study their ICU course and outcome.

Methods: Data from 93 consecutive ICU patients with candiduria, admitted during an 18-month period, were obtained retrospectively. Data regarding patient demographics, ICU course and outcome were entered in a pro forma.

Results: Out of 3,142 ICU admissions, the incidence of candidemia was $29.6 / 1,000$ admissions. A high proportion of patients $(80.6 \%)$ had an indwelling urinary catheter with the mean duration of catheter days being $5.89 \pm 3$ days. Other associated risk factors such as diabetes mellitus and antibiotic usage were seen in $74.2 \%$ and $86 \%$, respectively. Concomitant candidemia was seen in $19.4 \%$ of cases. Nonalbicans Candida spp. (66.7\%) emerged as the predominant pathogen causing candiduria. ICU mortality was $29 \%$ (Table 1).

Conclusion: There is a high incidence of candiduria in ICU patients, especially among those with indwelling catheters and those on antibiotic therapy. Moreover, in our cohort, an increased proportion of patients with candiduria had noncandida infection, emphasizing the need to have localized regional surveillance studies to identify the locally prevalent candida species and devise antifungal therapy protocols.

P20

Erythropoietin enhances the effects of transplanted mesenchymal stem cells in an experimental model of endotoxemia

A Sorokina ${ }^{1 *}$, A Averyanov$^{1}$, F Zabozlaev ${ }^{1}$, A Konoplyannikov², D Akulshin",

O Kuzovlev ${ }^{1}$

${ }^{1}$ Federal Research Clinical Center FMBA of Russia, Moscow, Russia;

${ }^{2}$ Radiologic Research Center, Obninsk, Russia

Critical Care 2012, 16(Suppl 3):P20

Background: Mesenchymal stem cells (MSCs) are able to reduce systemic inflammatory response in an experimental sepsis. One of the limiting factors of MSC therapy is a high degree of apoptosis of transplanted cells. On the basis of recently detected receptors for erythropoietin (EPO) on the surface of MSCs we hypothesized that introduction of EPO together with MSCs may increase survival of grafted MSCs and improve the clinical efficacy of cell transplantation.

Methods: Fifty Wistar male rats were randomized into five groups with 10 animals in each: Group 1 were healthy controls, Groups 2 to 5 were intraperitoneally introduced bacterial LPS $20 \mathrm{mg} / \mathrm{kg}$. Two hours later LPS 


\section{Table 1(abstract P20) Morphometric characteristics of the examined tissues in the experimental groups}

\begin{tabular}{llllll}
\hline & Controls & LPS & LPS + MSCs & LPS + EPO & LPS + EPO +MSCs \\
\hline Alveolar septal thickening $(\mu \mathrm{M})$ & $10.32 \pm 2.05$ & $31.25 \pm 2.9$ & $15.3 \pm 2.29^{*}$ & $28.6 \pm 4.1$ & $10.82 \pm 1.08^{* *}$ \\
Kidney tubular dystrophy (point) & 0 & $2.54 \pm 0.31$ & $2.22 \pm 0.29$ & $1.90 \pm 0.12^{*}$ & $0.74 \pm 0.19^{* *}$ \\
T-lymphocyte volume in thymus cortex (\%) & $86.24 \pm 2.88$ & $34.59 \pm 1.34$ & $49.37 \pm 2.16$ & $38.21 \pm 1.73$ & $81.48 \pm 2.63^{* *}$ \\
Spleen white pulp volume (\%) & $23.95 \pm 2.8$ & $11.15 \pm 3.8$ & $19.9 \pm 2.92$ & $22.8 \pm 0.98$ & $27.15 \pm 2.89^{*}$ \\
\hline
\end{tabular}

${ }^{*} P<0.05,{ }^{* *} P<0.01$ compared with LPS group.

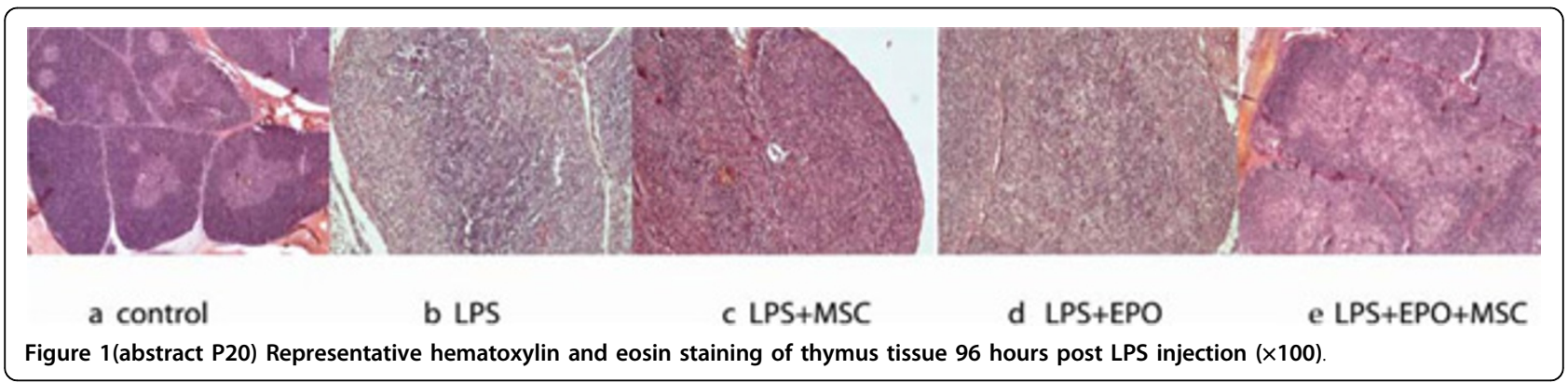

injection animals received the following intravenous treatment: $4 \times 10^{5}$ allogeneic MSCs (Group 3), $8.5 \mu \mathrm{g}$ recombinant EPO- $\beta$ (Group 4), MSCs and EPO in the same doses (Group 5). Surviving animals were euthanized on the fourth day. The morphological study, white blood cell count (WBC) and serum level of IL- $1 \beta, \mathrm{IL}-2, \mathrm{IL}-6$, and TNF $\alpha$ measurement were performed.

Results: The highest WBC was found in the group of combined treatment $E P O+M S C$. The serum IL- $1 \beta$ level in Groups 2 and 4 was significantly higher than in healthy and treated with MSCs and EPO + MSCs animals. The main results of the morphologic analysis of different tissues in the groups are presented in Table 1. The most significant differences in the LPS + EPO group were found in the lymphoid tissue - considerable hyperplasia of spleen white pulp and thymus cortex (Figure 1).

Conclusion: Combined treatment with EPO and MSCs can reduce acute lung injury and kidney damage, cause hyperplasia of lymphoid tissue and enhance the immune response more than separate treatment in an experimental model of endotoxemia in rats.

P21

Abstract withdrawn

Critical Care 2012, 16(Suppl 3):P21

\section{P22}

Abstract withdrawn

Critical Care 2012, 16(Suppl 3):P22

\section{P23}

Development and validation of a bedside prediction score for nosocomial sepsis in the pediatric ICU: a prospective observational cohort study

LG Saptharishi*, M Jayashree, S Singhi

PGIMER, Chandigarh, India

Critical Care 2012, 16(Suppl 3):P23

Background: Diagnosis of nosocomial sepsis (NS) is a challenge in every pediatric ICU $[1,2]$. There are very few studies on NS prediction in children, and those existing [3-8] have studied risk factors with emphasis on admission variables. In contrast, our study attempts to simplify the decisionmaking process using a dynamic scoring system based on objective criteria. Methods: This was a prospective study where 428 consecutive admissions, aged 1 month to 12 years, between January and October 2011 with PICU stay $>48$ hours, were enrolled and followed-up during their ICU stay and 72 hours thereafter. Occurrence of culture-positive nosocomial infections and relevant details were recorded. Patients with and without nosocomial sepsis were compared by chi-square test or Fisher's exact test for categorical and unpaired $t$ test or Kruskal-Wallis for continuous variables. Significant predictors of NS $(P<0.05$ on univariate analysis) were included in the binary backward stepwise logistic regression. The resultant derivation model's discrimination and calibration were assessed using the receiver operator characteristic (ROC) curve and Hosmer-Lemeshow test, respectively. The final model was transformed into a score, based on the regression coefficients. For internal validation, bootstrapping and shrinkage coefficients were used. Results: Of the 428 enrolled, 17 were excluded (malignancies (14 cases), burns (one case), polytrauma (one case) and missing data (one case)). A total of 151 episodes (23.1\%; 95 out of 411 children) of culture-positive NS were seen giving an incidence rate of 4.5 per 100 patient-days. Age, PRISM III score, device utilization, albumin, immunomodulator and prior antibiotic use, and intubation were significant independent predictors on multivariate analysis (Table 1). This model had an AUC-ROC of 0.87 (Figure 1). Also, the Hosmer-Lemeshow chi-square was $5.06(P=0.75)$ indicating good fit of the model. Based on the regression coefficients, a pediatric nosocomial sepsis prediction score (Pe-NoSeP) was developed. Probability cutoffs versus sensitivity and specificity plotting showed a cutoff of 0.38 corresponding to a score of 15 . The sensitivity, specificity, positive predictive value, negative predictive value and positive likelihood ratio at this cutoff were $79.1 \%$, $79.1 \%, 61.6 \%, 89.9 \%$ and 3.76 , respectively. The accuracy of the model was $79.3 \%$ and reduced classification errors from $29.8 \%$ to $20.7 \%$. All seven predictors retained their statistical significance after bootstrapping, confirming the validity of the score.

Conclusion: The Pe-NoSeP score is a simple, easy-to-use bedside prediction model, which estimates the probability of NS in a child on a particular day and assists clinical decision-making. This tool may have diagnostic, therapeutic and preventive utilities based on its application.

\section{References}

1. Fischer JE: Physician's ability to diagnose sepsis in newborns and critically ill children. Pediatr Crit Care Med 2005, 6(Suppl):S120-S125.

2. Pauker SG, Kopelman Rl: Interpreting hoof-beats: can Bayes help clear the haze? N Engl J Med 1992, 327:1009-1013.

3. De Mello MJG, de Albuquerque PM, Lacerda HR, Barbosa MTS, Ximenes RA: Risk factors for healthcare-associated infection in a pediatric intensive care unit. Pediatr Crit Care Med 2010, 11:246-252. 
Table 1 (abstract P23) Multivariate logistic regression analysis: independent predictors and the Pediatric Nosocomial Sepsis Prediction Score (Pe-NoSeP)

\begin{tabular}{|c|c|c|c|c|c|c|c|}
\hline Serial no. & Variable & B Coeff. & Odds ratio (OR) & OR $95 \% \mathrm{Cl}$ & $P$ value & Category & Score \\
\hline \multirow[t]{5}{*}{1} & PRISM III score & 0.06 & 1.06 & 1.02 to 1.10 & 0.003 & 5 to 15 & 0 \\
\hline & & & & & & 16 to 26 & 2 \\
\hline & & & & & & 27 to 37 & 4 \\
\hline & & & & & & 38 to 48 & 7 \\
\hline & & & & & & 49 to 59 & 9 \\
\hline \multirow[t]{2}{*}{2} & Indwelling catheter use & 1.67 & 5.31 & 2.31 to 12.23 & $<0.001$ & No & 0 \\
\hline & & & & & & Yes & 6 \\
\hline \multirow[t]{2}{*}{3} & Albumin transfusion & 1.35 & 3.87 & 1.58 to 9.50 & 0.003 & No & 0 \\
\hline & & & & & & Yes & 5 \\
\hline \multirow[t]{2}{*}{4} & Age ( $\leq 5$ years/ $>5$ years) & 0.90 & 2.45 & 1.22 to 4.93 & 0.010 & $>5$ years & 0 \\
\hline & & & & & & $\leq 5$ years & 3 \\
\hline \multirow[t]{2}{*}{5} & Immunomodulator use & 1.30 & 3.66 & 1.23 to 10.94 & 0.020 & No & 0 \\
\hline & & & & & & Yes & 5 \\
\hline \multirow[t]{2}{*}{6} & Intubation & 1.70 & 5.48 & 2.53 to 11.88 & $<0.001$ & No & 0 \\
\hline & & & & & & Yes & 6 \\
\hline \multirow[t]{2}{*}{7} & Prior antibiotic use $(<4 / \geq 4)$ & 0.58 & 1.79 & 1.03 to 3.11 & 0.040 & $<4$ & 0 \\
\hline & & & & & & $\geq 4$ & 2 \\
\hline
\end{tabular}

4. Singh-Naz N, Spraque BM, Patel KM, Pollack MM: Risk factors for nosocomial infection in critically ill children: a prospective cohort study. Crit Care Med 1996, 24:875-878.

5. $\quad$ Singh-Naz N, Sprague BM, Patel KM, Pollack MM: Risk assessment and standardized nosocomial infection rate in critically ill children. Crit Care Med 2000, 28:2069-2075.
6. Morrow LE, Kollef MH: Recognition and prevention of nosocomial pneumonia in the intensive care unit and infection control in mechanical ventilation. Crit Care Med 2010, 38(8 Suppl):S352-S356.

7. Bota DP, Melot C, Ferreira FL, Vincent JL: Infection Probability Score (IPS): a method to help assess the probability of infection in critically ill patients. Crit Care Med 2003, 31:2579-2584.

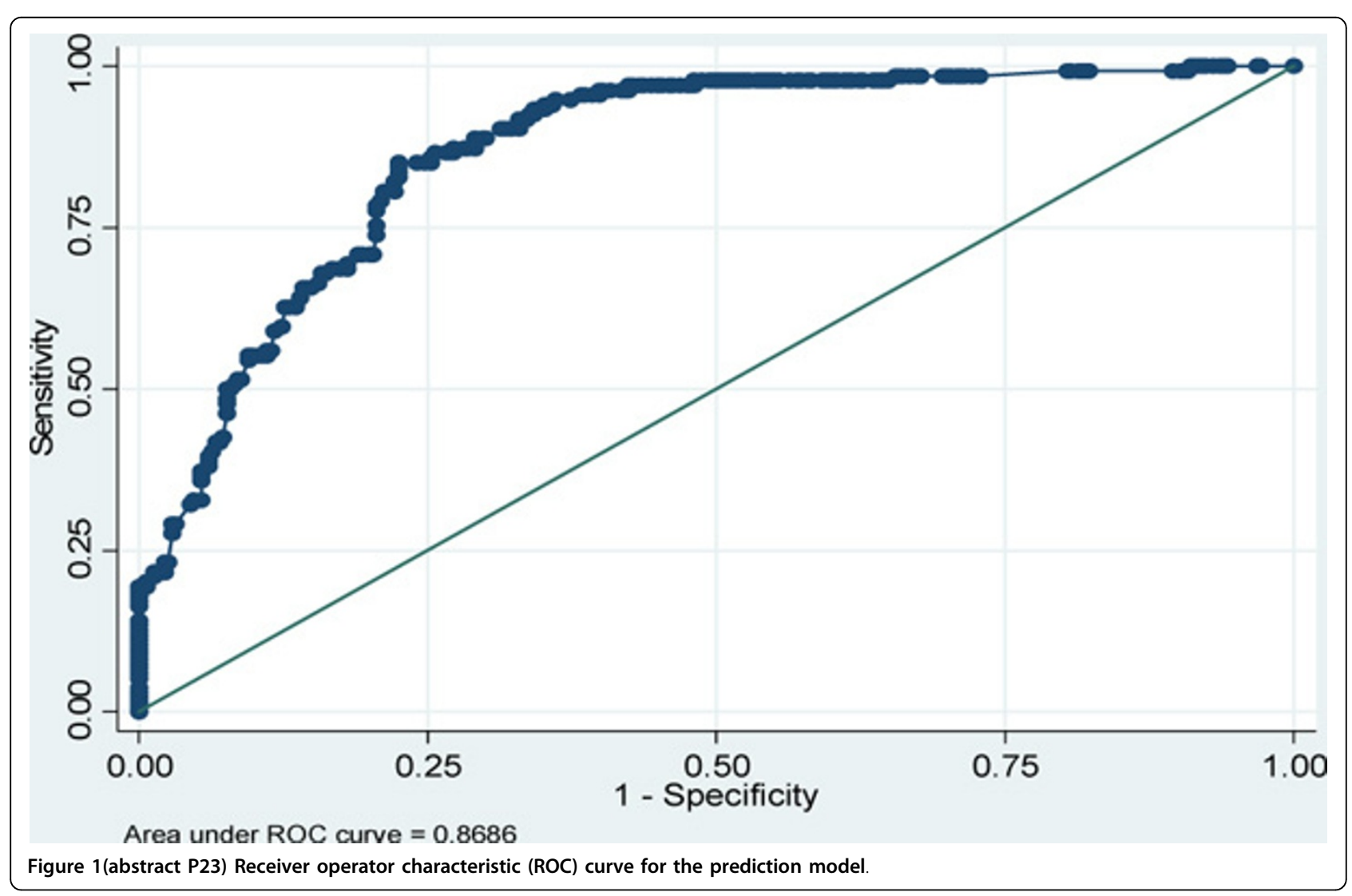


8. Arantes A, Carvalho ES, Medeiros EAS, Farhat CK, Mantese OC: Pediatric risk of mortality and hospital infection. Infect Control Hosp Epidemiol 2004, 25:783-785.

P24

Effects of statins on mitochondrial respiration and outcome during experimental sepsis

J Morel $^{1 *}$, I Hargreaves ${ }^{2}$, B Bollen-Pinto ${ }^{3}$, D Brealey ${ }^{3}$, J Backman ${ }^{4}$, A Dyson $^{3}$, M Singer ${ }^{3}$

${ }^{1} \mathrm{CHU}$ Saint Etienne, Saint Etienne, France; ${ }^{2}$ Neurometabolic Unit, National Hospital for Neurology and Neurosurgery Queen Square, London, UK;

${ }^{3}$ Bloomsbury Institute of Intensive Care Medicine, University College of London, UK; ${ }^{4}$ University of Helsinki and HUSLAB Helsinki University Central

Hospital, Helsinki, Finland

Critical Care 2012, 16(Suppl 3):P24

Background: Statins target several mechanisms involved in the pathophysiology of sepsis, leading to their consideration as an adjuvant therapy. Ubiquinone is an important mitochondrial antioxidant and constituent of the electron transport chain. Ubiquinone production is inhibited by statins, whereas sepsis itself also affects mitochondrial activity. The impact of statins on mitochondrial function in sepsis has not been previously explored.

Methods: Sepsis was induced in instrumented, awake, male Wistar rats by i.p. injection of faecal slurry. Fluid resuscitation was provided by continuous intravenous infusion. Simvastatin $20 \mathrm{mg} / \mathrm{kg}$ bd was administrated by gavage commencing either 3 days pre-sepsis (pre-treatment) or from 6 hours postsepsis (post-treatment). A control group received only vehicle but no active drug (vehicle). Survival was assessed at 72 hours (16 per group). In a second set of experiments, rats were sacrificed at 24 hours post-sepsis (seven per group) for measurement of: plasma biochemistry and simvastatin acid; heart and muscle ubiquinone levels by HPLC; and oxygen consumption on permeabilized soleus muscle fibers in a Clark electrode chamber. A fourth group of naive animals was also used as healthy controls. Statistics were performed using the Wilcoxon test and repeated-measures ANOVA and post hoc Bonferroni test.

Results: Survival at 72 hours (Figure 1) was $43.7 \%, 25 \%$ and $12.5 \%$ for pre-treatment, vehicle, and post-treatment groups, respectively $(P<0.05)$. At 24 hours post sepsis, ubiquinone was significantly decreased only in hearts taken from statin pre-treated animals (706 \pm 222 vs. 1,217 \pm 269 $\mathrm{pmol} / \mathrm{mg}$ protein for vehicle hearts). Plasma simvastatin acid levels were significantly increased in statin pre-treated animals $(41 \pm 3 \mathrm{ng} / \mathrm{ml}$ ) compared with naïve animals receiving the same dose $(1 \pm 0.4 \mathrm{ng} / \mathrm{ml}$ ). Compared with vehicle-treated animals, statin pre-treatment resulted in significant decreases in urea and creatinine, and a trend towards improvement for liver enzymes and creatine kinase. Plasma lipid levels were not significantly affected by statins given either before or after the onset of sepsis. Mean values of muscle oxygen consumption were $11.9 \pm 5.3$, $14.4 \pm 2.9,12.1 \pm 1.5$ and $5.9 \pm 2.4 \mathrm{pmol} / \mathrm{ml} / \mathrm{second} / \mathrm{mg}$ for naïve, sepsis + post-treatment, sepsis + pre-treatment and sepsis + vehicle groups,

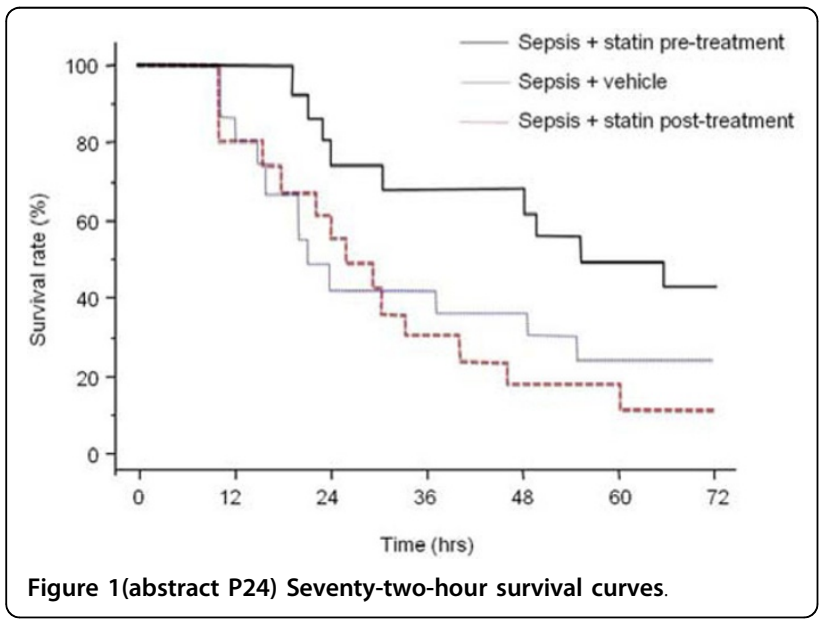

respectively. The significant reduction in muscle oxygen consumption seen in the sepsis + vehicle group was prevented in both groups receiving simvastatin.

Conclusion: This study confirms the beneficial effect of statins when given before the onset of sepsis, and this appear to be independent of its lipidlowering property. This beneficial effect is likely to be multifactorial but could be attributed in part to a protective effect on mitochondrial respiration.

P25

Abstract withdrawn

Critical Care 2012, 16(Suppl 3):P25

\section{Abstract withdrawn:}

\section{P26}

Effect of phenolic acids originating from microbes on mitochondria and neutrophils

NV Beloborodova ${ }^{1 *}$, AY Olenin ${ }^{2}$, NI Fedotcheva ${ }^{3}$, V Shubina $^{3}$, W Teplova ${ }^{3}$ 'Negovsky Research Institute of General Reanimatology, Russian Academy of Medical Sciences, Moscow, Russia; ${ }^{2}$ Lomonosov Moscow State University, Moscow, Russia; ${ }^{3}$ Institute of Theoretical and Experimental Biophysics, Russian Academy of Sciences, Pushchino, Moscow Region, Russia Critical Care 2012, 16(Suppl 3):P26

Background: Several low-molecular-weight phenolic acids are present in the blood of septic patients at high levels [1,2]. The microbial origin of the most of phenylcarboxylic acids in the human body was shown previously [3], but the pathophysiological role of phenolic acids is not clear. It was shown that microbial phenolic acids produce either the antioxidant or the pro-oxidant action on mitochondria depending on the chemical structure [4]. In this work the influence of phenolic acids on reactive oxygen species (ROS) production in mitochondria and neutrophils was investigated.

Methods: Mitochondria were isolated from the liver of Wistar rats. The difference of electric potentials on the inner mitochondrial membrane $\left(\Delta \psi_{M}\right)$ was determined from the distribution of the lipophilic cation of tetraphenylphosphonium whose concentration in external medium $\left[\mathrm{TPP}^{+}\right]_{\text {out }}$ was registered using a $\mathrm{TPP}^{+}$-selective electrode. The rate of oxygen consumption by mitochondria was determined polarographically. The production of ROS by mitochondria was determined by measuring the chemiluminescence of the Cypridina luciferin. The generation of superoxide anion was induced by $25 \mu \mathrm{M}$ menadione. The production of ROS by neutrophils was examined by the method of luminol-dependent chemiluminescence. All reagents used were from Sigma (USA). Statistical processing of the data was carried out using the program MS Excel 2003. The differences were considered significant at $P \leq 0.05$.

Results: ROS production by mitochondria was higher in the presence of cinnamic acid, benzoic acid, 3-phenylpropionic acid and phenylacetic acid than in the presence of menadione alone. The most effective activators of ROS production in mitochondria were those phenolic acids whose effect is mediated via the interaction with thiol $(-\mathrm{SH})$ groups. ROS production by mitochondria was lower in the presence of phenyllactate, $p$-hydroxyphenylacetate or $p$-hydroxyphenyllactate compared with the control. All phenolic acids inhibited ROS formation in neutrophils to different degrees. The inhibition was related to the scavenging of the superoxide anion.

Conclusion: Microbial metabolites from both the normal gut microbiota and infection sources enter the circulation and can enhance or reduce the inflammatory response. In healthy people phenyllactate and $p$ hydroxyphenyllactate, which decrease ROS production in both in mitochondria and neutrophils, can have the protecting effect on organs and tissues. They can play a role of natural antioxidants. But in septic patients deficit phenolic acids producing the pro-oxidant effects on mitochondria (for example, PPA and PAA) and high levels of other phenols can led to mitochondrial dysfunction and multiorgan failure. Acknowledgements: This work was supported by the Russian Ministry of Education and Science (Project No. 16.512.11.2227). 
References

1. Beloborodova NV, Osipov GA: Small molecules originating from microbes (SMOM) and their role in microbes-host relationship. Microbial Ecol Health Dis 2000, 12:12-21

2. Khodakova AS, Beloborodova NV: Microbial metabolites in the blood of patients with sepsis. Crit Care 2007, 11:P5.

3. Beloborodova NV, Khodakova AS, Bairamov IT, Olenin AY: Microbial origin of phenylcarboxylic acids in the human body. Biochemistry (Moscow) 2009, 74:1350-1355.

4. Fedotcheva NI, Kazakov RE, Kondrashova MN, Beloborodova NV: Toxic effects of microbial phenolic acids on the functions of mitochondria. Toxicol Lett 2008, 180:182-188.

P27

Immunological modulation of estrogen during sepsis

AM Stabile*, ME Batalhão, EC Carnio

University of Sao Paulo, College of Nursing at Ribeirão Preto, Ribeirão Preto, Brazil

Critical Care 2012, 16(Suppl 3):P27

Background: Sepsis and its common complication septic shock, are generally induced by the action of lipopolysaccharide (LPS) and is characterized by peripheral arteriolar vasodilatation which results in hypotension and inadequate tissue perfusion. Nitric oxide (NO) is a free radical gas, produced by the immune system in response to an immunological stimulus and is related to the pathogenesis of sepsis due to its vasodilator and cytotoxic actions. One significant finding in clinics is that man and woman respond differently to sepsis, with better prognosis related to women [1].

Objective: This study was designed to investigate the role of estrogen on immune response in an experimental model of septic shock.

Methods: In the first set of experiments male and female (ovariectomized and sham surgery) rats were injected intraperitoneally (IP) for three consecutive days with estradiol cypionate (ECP), $40 \mu \mathrm{g} / \mathrm{kg}$ or vehicle. In the third day, after ECP injection, rats receive IP injection of $10 \mathrm{mg} / \mathrm{kg}$ of bacterial LPS or saline solution. Plasma was collected 4 and 6 hours after LPS. In the second set of experiments macrophage culture was performed from peritoneal wash of male and female rats. Culture was stimulated with $\beta$-estradiol $\left(10^{-9} \mathrm{M}\right)$ and LPS $1 \mu \mathrm{g} / \mathrm{ml}$ and medium collected 12 and 24 hours after stimulation for NO measure.

Results: In vivo experiments showed that administration of LPS increased NO plasma concentration in males and females. ECP pre-treatment decreased NO concentration in sham females in the two studied periods (4 and 6 hours); conversely, increased nitrate levels in ovariectomized and had no effect in males. Results of in vitro experiments showed that macrophages from males produced more NO than the ones from female, either in basal conditions or after LPS stimulation; however, treatment of the culture with $\beta$-estradiol, significantly increased the NO production in macrophages from male but had no effect in female rats.

Conclusion: Our results indicate that estradiol may have pro or antiinflammatory actions depending on the gender; however, estradiol in female seems to be protective, since it decreased NO plasma concentration. These results may explain in part the better outcomes of woman during sepsis.

\section{Acknowledgements: FAPESP}

\section{Reference}

1. Martin GS, Mannino DM, Eaton S, Moss M: The epidemiology of sepsis in the United States from 1979 through 2000. N Engl J Med 2003, 348:1546-1554.

\section{P28}

Use of Centre for Disease Control criteria to classify infections in critically ill patients: results from an interobserver agreement study PMC Klein Klouwenberg ${ }^{1 *}$, DSY Ong ${ }^{1}$, LD Bos ${ }^{2}$, FM de Beer ${ }^{2}$, MA Huson ${ }^{2}$, M Straat ${ }^{2}$, LA van Vught ${ }^{2}$, L Wieske ${ }^{2}, \mathrm{~J} \mathrm{Horn}^{2}$, MJ Schultz ${ }^{2}, \mathrm{~T}$ van der Poll ${ }^{2}$, MJM Bonten ${ }^{1}$, OL Cremer $^{1}$

'University Medical Centre, Utrecht, the Netherlands; ${ }^{2}$ Academic Medical Centre, Amsterdam, the Netherlands

Critical Care 2012, 16(Suppl 3):P28

\section{Table 1(abstract P28) Agreement across the various} sources of infection

\begin{tabular}{ll}
\hline Source of infection & Agreement/total number \\
\hline Lower respiratory tract & $86 / 90(96 \%)$ \\
Community-acquired pneumonia & $28 / 32(88 \%)$ \\
Hospital-acquired pneumonia & $32 / 39(82 \%)$ \\
Ventilator-acquired pneumonia & $12 / 17(71 \%)$ \\
Abdominal & $34 / 36(91 \%)$ \\
Secondary peritonitis & $21 / 24(88 \%)$ \\
Primary peritonitis & $2 / 2(100 \%)$ \\
Intra-abdominal abscess & $3 / 5(70 \%)$ \\
Bloodstream & $30 / 34(94 \%)$ \\
Line infection & $22 / 26(85 \%)$ \\
Primary bloodstream infection & $4 / 5(80 \%)$ \\
Central nervous system & $10 / 11(91 \%)$ \\
Secondary meningitis & $5 / 7(79 \%)$ \\
Primary meningitis & $4 / 4(100 \%)$ \\
\hline
\end{tabular}

Background: Correct classification of the source of infection is important in observational and interventional studies of sepsis. The Centre for Disease Control (CDC) criteria are most commonly used for this purpose, but the robustness of these definitions in critically ill patients is not known. We determined the interobserver agreement for classifying infections in the ICU.

Methods: Data were collected as part of a prospective cohort of 1,214 critically ill patients admitted to two hospitals in the Netherlands between January 2011 and June 2011. Eight observers assessed a random sample of 168 out of 554 patients who had experienced at least one infectious episode in the ICU. Each patient was assessed by two randomly selected observers who independently scored the source of infection (by affected organ system or site), the plausibility of infection (rated as none, possible, probable, or definite), and the most likely causative pathogen. Assessments were based on a post hoc review of all available clinical, radiological and microbiological evidence. The observed diagnostic agreement for source of infection was classified as partial (that is, matching on organ system or site) or complete (that is, matching on specific diagnostic terms), for plausibility as partial (two-point scale) or complete (four-point scale), and for causative pathogens as an approximate or exact pathogen match. Interobserver agreement was expressed as percentage agreement and as a kappa statistic. Results: A total of 206 infectious episodes were observed in 168 patients. Agreement regarding the source of infection was $89 \%(183 / 206)$ and $69 \%$ $(142 / 206)$ for partial and complete diagnostic agreement, respectively (Table 1). This resulted in an overall kappa of $0.85(95 \% \mathrm{Cl}=0.79$ to 0.90$)$. Agreement varied from 70 to $100 \%$ within major diagnostic subgroups. In the subgroup of 142 episodes with full diagnostic agreement on source of infection, the interobserver agreement for plausibility of infection was $83 \%$ and $65 \%$ on a two-point and four-point scale, respectively. For causative pathogen, agreement was $78 \%$ and $70 \%$ for an approximate and exact pathogen match, respectively.

Conclusion: In this study, overall interobserver agreement of classifying infections using CDC criteria was excellent, whereas an exact match on all aspects of the diagnosis between independent observers was limited for some infections.

P29

Patients with sepsis exhibit mitochondrial biogenesis in peripheral blood immune cells

F Sjövall, S Morota, MJ Hansson, E Elmér

Lund University, Lund, Sweden

Critical Care 2012, 16(Suppl 3):P29

Background: Sepsis is one of the leading causes of admission to the ICU. After the initial proinflammatory response a gradual change towards a more anti-inflammatory pattern can be seen. In this latter stage, immune 
cell function has been suggested to be downregulated leading to an immunoparalysis, lending the patient more vulnerable to deleterious secondary infections. Mitochondrial dysfunction has been suggested to play a role in this immunoparalytic state and we therefore investigated mitochondrial respiratory function in peripheral blood immune cells (PBICs) in patients with sepsis and its evolvement over time.

Methods: Twenty patients with severe sepsis or septic shock were included. PBICs were isolated from freshly drawn blood via density gradient centrifugation and analyzed three times during the first week after admission to the ICU (within 48 hours, days 3 to 4 and days 6 to 7). Mitochondrial respiration was examined with high-resolution respirometry in intact and permeabilized cells in order to evaluate both whole cell respiration as well as contribution of individual complexes. Mitochondrial DNA (mtDNA), cytochrome $c$ (Cyt $c)$ and citrate synthase (CS) were measured as indicators of change in cellular mitochondrial content.

Results: In intact PBICs from septic patients, with endogenous substrates, there was a gradual increase in cellular respiration that was $73 \%$ higher after 1 week compared with controls $(P=0.003)$. In permeabilized cells, complex I, II and IV displayed increased respiration compared with controls already at days 1 to $2(37 \%, 30 \%$ and $73 \%$ respectively, $P<0.001)$ and continued to increase to days 6 to 7 (68\%, 68\% and $108 \%$ respectively). The rise in mitochondrial respiration was paralleled by higher levels of CS activity and increased mtDNA and Cyt $c$ content in cells from septic patients $(90 \%, 143 \%$ and $231 \%$ for the respective parameter at days 6 to 7 compared with controls, $P<0.0001)$. Mortality for the septic patients was $25 \%$ by day 7 . There was no difference in respiratory capacity between survivors and nonsurvivors at any of the time points measured.

Conclusion: In PBICs from patients with severe sepsis or septic shock there is a gradual increase in mitochondrial respiratory capacity. This increase is probably due to mitochondrial biogenesis as indicated by increases in mitochondria-specific markers. Nonsurvivors displayed the same increase in respiration as survivors arguing against mitochondrial respiratory dysfunction and defect mitochondrial biogenesis, in PBICs, as a mediator of increased mortality in the septic condition.

\section{P30}

AMP-activated protein kinase preserves endothelial tight junctions in the coronary microcirculation during sepsis

D Castanares-Zapatero ${ }^{1 *}$, C Bouleti ${ }^{2}$, T Mathivet ${ }^{2}$, B Gerber' $^{1}$, C Oury $^{3}$,

L Bertrand ${ }^{1}$, JL Vanoverschelde ${ }^{1}$, PF Laterre ${ }^{4}$, S Horman ${ }^{1}$, C Beauloye

${ }^{1}$ Université catholique de Louvain, Institut de recherche expérimentale et clinique (pôle cardiovasculaire), Brussels, Belgium; ${ }^{2}$ Collège de France, Centre Interdisciplinaire de Recherche en Biologie INSERM 1050, Paris, France; ${ }^{3}$ Université de Liège, Groupe Interdisciplinaire de Génoprotéomique Appliquée, Liège, Belgium; ${ }^{4}$ Université catholique de Louvain, Cliniques universitaires Saint Luc Intensive Care Unit, Brussels, Belgium Critical Care 2012, 16(Suppl 3):P30

Background: A compromising endothelial cell (EC) monolayer affects vascular permeability and leads to fluid extravasation. Tight junctions and more particularly Zonula occludens 1 (ZO-1) play a major role in maintaining vascular barrier integrity and are regulated by cytoskeletal proteins. During sepsis, endothelial barrier disruption occurs in most organs and contributes to organ dysfunction. We and others have demonstrated that the $\alpha_{1}$ isoform of AMP-activated protein kinase ( $\alpha_{1}$ AMPK) controls cytoskeleton organisation in various cell types including endothelial cells. Therefore, we hypothesised that $\alpha_{1}$ AMPK preserves tight junction organisation and vascular permeability during sepsis in the coronary microcirculation.

Methods: In vitro, tight junction organisation (ZO-1 staining), cytoskeleton organisation (phalloïdin staining) and vascular permeability were measured in endothelial cells in culture (HCAECs). Endothelial cells were pretreated with $1 \mathrm{mM}$ AICA riboside (AICAr) before LPS challenge (50 $\mu \mathrm{g} / \mathrm{ml}$ O55:B5). In vivo, wild-type $\left(\alpha_{1} \mathrm{AMPK}^{+/+}\right)$mice were treated with LPS (O55:B5,10 mg/kg) and compared with $\alpha_{1}$ AMPK knockout animals $\left(\alpha_{1} \mathrm{AMPK}^{-1}\right)$. ZO-1 localisation was determined on frozen heart sections. Vascular permeability was evaluated using Evans Blue dye leakage. In addition, myocardial wall oedema was assessed by magnetic resonance imaging (MRI).

Results: In vitro, LPS-challenged cells displayed a significant disruption of the ZO-1 linear configuration after 24 hours and exhibited a decrease in peripheral actin filaments. Gap areas appeared in the cellular monolayer exposed to LPS, unlike untreated cells for which the monolayer remained unaltered. Consequently, LPS treatment gradually increased endothelial cells monolayer permeability in a time-dependent manner. AMPK activation by AICAr preserved the linear pattern of ZO-1 and prevented gap areas in the monolayer. More interestingly, AMPK activation reduced LPS-induced hyperpermeability. In vivo, according to the in vitro experiments, a dramatic decrease in ZO-1 staining was observed in $\alpha_{1} \mathrm{AMPK}^{-/-}$hearts compared with $\alpha_{1} \mathrm{AMPK}^{+/+}$, after LPS challenge. This resulted in an increased Evans Blue extravasation and, more importantly, in an exaggerated myocardial wall oedema observed by MRI. Finally, treating C57BI6 mice with AICAr reduced cardiac vascular hyperpermeability in our model of sepsis.

Conclusion: The AMPK signalling pathway protects the endothelial barrier during sepsis by preserving tight junction organisation. Since AMPK counteracts the molecular events involved in the LPS-induced barrier disruption in coronary microcirculation, it could consequently represent a new therapeutic molecule during sepsis.

\section{P31}

Weibel-Palade body exocytosis as a therapeutic target to improve hemodynamics in Gram-positive sepsis

JY Lee ${ }^{*}$, HM Linge ${ }^{2}$, K Ochani ${ }^{2}$, YZ Zhang ${ }^{2}$, EJ Miller ${ }^{3}$

${ }^{1}$ The Elmezzi Graduate School of Molecular Medicine, Manhasset, NY, USA;

${ }^{2}$ The Feinstein Institute for Medical Research, Heart and Lung Research

Center, Manhasset, NY, USA; ${ }^{3}$ Hofstra University School of Medicine, Heart and Lung Research Center, Manhasset, NY, USA

Critical Care 2012, 16(Suppl 3):P31

Background: Gram-positive bacterial lung infection is commonly associated with sepsis, and particularly important in older individuals. Systemic vascular dysfunction associated with sepsis is characterized by vascular permeability that can lead to tissue hypoxia. Our studies focus on angiopoietin-2 (Ang-2), whose increased plasma concentration is associated with severity of lung injury and with mortality. Ang-2 is stored in the Weibel-Palade body (WPB), an endothelial-specific secretory organelle. Here, we examine the release of Ang-2 from primary human pulmonary microvascular endothelial cells (HPMECS) in vitro stimulated with Gram-positive bacterial cell wall components. We then used our model of pulmonary infection to investigate the use of an inhibitor of WPB exocytosis to prevent systemic inflammation and hemodynamic instability.

Methods: HPMECs were treated with lipoteichoic acid (LTA; 50 to $100 \mu \mathrm{g} / \mathrm{ml}$ ) and peptidoglycan (PGN; 167 to $333 \mu \mathrm{g} / \mathrm{ml}$ ) in the presence or absence of TAT-NSF700 fusion peptide $(10 \mu \mathrm{M})$ to inhibit WPB exocytosis. Ang-2 in culture medium was measured by ELISA, and WPB exocytosis assessed using immunofluorescent (IF) staining of Ang-2. HPMEC monolayer permeability was measured using FITC-dextran. Male C57/BI6 mice, 8 to 10 weeks old $(n=6 /$ group $)$, were pretreated with TAT-NSF700 $(0.5 \mathrm{mg} / \mathrm{kg})$ or saline intraperitoneally. Thirty minutes later, LTA $(150 \mu \mathrm{g})$ and PGN $(500 \mu \mathrm{g})$ or saline alone were instilled intratracheally. Pulse oximetry was assessed in awake mice prior to and 6 hours post instillation. The mice were then euthanized by exsanguination under anesthesia and bronchoalveolar lavage (BAL) was performed. BAL fluid total and differential counts were evaluated and protein and cytokine concentrations in plasma and BAL were assessed using commercial assays.

Results: LTA-PGN increased Ang-2 levels dose dependently (up to ninefold, $P=0.003$ ) in HPMEC culture medium within 30 minutes, which was blocked by TAT-NSF700 pretreatment. IF staining showed aggregation and localization of Ang-2 in the cytoplasm suggesting WPB exocytosis within 30 minutes. LTA-PGN also induced HPMEC permeability. LTA-PGN induced both local and systemic inflammation resulting in decreased heart and breath rates and oxygen saturation ( $94 \%$ vs. $97 \%, P=0.038)$ at 6 hours. These physiological changes were prevented in mice pretreated with TAT-NSF700.

Conclusion: LTA-PGN induced rapid Ang-2 secretion via WPB exocytosis and this release is associated with significant changes in permeability. In vivo, LTA-PGN induces significant changes in heart and breath rates and oxygen saturation that was prevented by inhibition of WPB exocytosis. Thus, we have defined WPB, a storage organelle for multiple proinflammatory mediators, as a potential target to control overwhelming inflammation in Gram-positive sepsis and improve tissue oxygenation and hemodynamics. 
P32

Effectiveness of nebulized amphotericin B to eradicate Candida colonization from the lower respiratory tracts of ICU patients

DSY Ong *, PMC Klein Klouwenberg, MJM Bonten, OL Cremer

University Medical Centre Utrecht, the Netherlands

Critical Care 2012, 16(Suppl 3):P32

Background: Candida species are opportunistic pathogens that are ordinarily found in the human gastrointestinal tract. In critically ill patients receiving mechanical ventilation, colonization of the lower respiratory tract (LRT) with Candida may occur in 25 to $55 \%$ of patients. Nebulized amphotericin B (NAB) is commonly used to eradicate Candida from the LRT, for example as part of selective decontamination of the digestive tract protocols. However, the clinical effectiveness of this approach is unknown. Our aim was to determine the time to eradication of Candida from the LRT in mechanically ventilated ICU patients receiving and not receiving inhalation therapy with $\mathrm{NAB}$.

Methods: We included patients admitted to the ICU of the University Medical Center Utrecht from November 2007 until February 2012. We excluded patients with a length of stay $<72$ hours and patients receiving systemic antifungal treatment. Microbiological screening for Candida colonization was performed on admission and twice weekly, and samples were processed according to a standardized protocol. Samples obtained in the first 72 hours of ICU admission were discarded since positive samples obtained on admission were not an indication to start amphotericin B. Colonization was defined as the presence of Candida in two or more consecutive samples obtained on different days. Decolonization was defined as the absence of Candida in two consecutive samples, or as the absence of Candida in the last available sample before extubation or discharge. Only the first episode of Candida colonization per admission was used for analysis. Hazard ratios of eradication of Candida colonization in treated patients compared with nontreated patients were determined using Cox regression analysis.

Results: Out of a total of 2,948 patients who were admitted for at least 72 hours, 288 were colonized with Candida. Concurrent systemic antifungal treatment during the colonization period was administered to 27 of these patients, who subsequently were excluded, leaving 261 patients for analysis. Decolonization occurred in 36 of 48 (75\%) and 112 of $213(53 \%)$ patients who received or did not receive $N A B$, respectively ( $\mathrm{HR} 1.51 ; 95 \% \mathrm{Cl}=1.03$ to 2.20 ). Median time to decolonization was 6.0 (IQR 5.6 to 6.4) and 9.0 days (IQR 8.1 to 10.9) in patients receiving and not receiving $N A B$, respectively (log-rank test, $P=0.03$ ) (Figure 1). Adjustment for age, sex and time from ICU admission to acquirement of Candida did not alter the results.

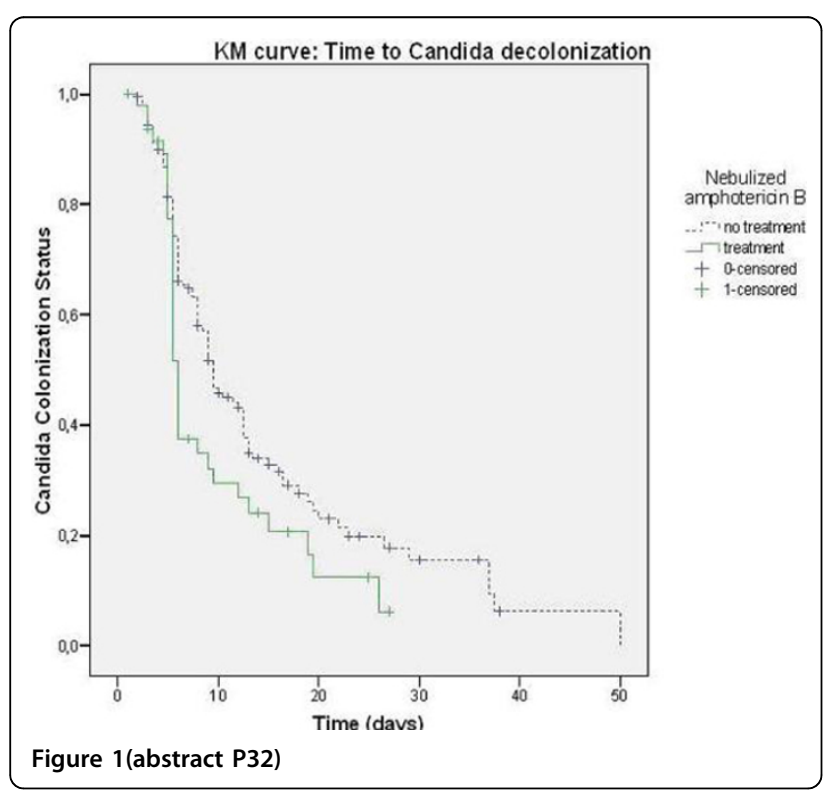

Conclusion: Inhaled amphotericin B treatment in mechanically ventilated patients with acquired Candida colonization of the lower respiratory tract significantly increases the rate of decolonization.

P33

Clinical and diagnostic significance of apoptosis in the development of neutropenia and bacterial complications in newborns with respiratory distress syndrome

M Puchtinskaya

Research Institute of Obstetrics and Pediatrics, Rostov-on-Don, Russia Critical Care 2012, 16(Suppl 3):P33

Background: The development of neutropenia with neonates with RDS on mechanical ventilation is a sign of the realization of bacterial complications $[1,2]$. The purpose of this study was the optimization of prevention of neonatal sepsis.

Methods: With permission of the Ethics Committee 64 full-term newborns with RDS on mechanical ventilation, without clinical signs of infection, were retrospectively divided into two groups: with a decrease in absolute neutrophil count $\left(\mathrm{M}<2,000\right.$ cells $/ \mathrm{mm}^{3}$ ) after 3 to 5 days of hospitalization $(\mathrm{I}, n=30)$ and non-neutropenic (II, $n=34)$. The survey was conducted at admission and 3 to 5 days. They were studied on the content of lymphocytes in the early (AnnexinV-FITC ${ }^{+} \mathrm{PI}^{-}$) and late (AnnexinV-FITC ${ }^{+} \mathrm{PI}^{+}$) apoptosis by flow cytometry (AnnexinV ${ }^{+}$-labeled FITK and propidium iodide $\left(\mathrm{PI}^{+}\right)$-labeled $\mathrm{PE}$; Saltag, USA), taking into account results on the cytometer (Beckman Coulter Epics XL, USA); the plasma level of granulocyte colony-stimulating factor (GCSF), fibroblast growth factor (FGF), anti-apoptosis soluble sFas-ligand (sFas-L) by ELISA (Victor, Finland; test system Cytimmune Sciences Inc., USA and Bender MedSystems GmbH, Austria). Points of cutoff were determined by ROC analysis. The statistical power of the study is $80 \%(\alpha \leq 0.05)$.

Results: At admission, it was revealed that the patients in group I have a high content of lymphocytes in the early and late apoptosis, and low levels of GCSF, FGF, sFas-L in reference to group II ( $\alpha \leq 0.05)$. In group I, 27 patients at 3 to 5 days developed neutropenia $\left(M<2,000\right.$ cells $/ \mathrm{mm}^{3}$ ) and a reduction in plasma levels of GCSF, FGF, sFas-L and an increase in lymphocyte apoptosis $(\alpha \leq 0.05)$. Sepsis developed in 22 children of group I and in only five patients of group II $(\alpha \leq 0.05)$. The values of cutoff (sensitivity, specificity, accuracy) for prediction of neutropenia in neonates with RDS at admission to the ICU were: for GCSF - 1,556 pg/ml $(85.1 \%$, $75.6 \%, 79.6 \%$, respectively); for sFAS-L - 5,870 pg/ml $(69.56 \%, 74.4 \%, 71.9 \%)$; for FGF - $25.7 \mathrm{pg} / \mathrm{ml}(68 \%, 82 \%, 74.4 \%)$; for early apoptosis - 9.59\% (82\%, $93 \%, 74 \%)$; and for late apoptosis - $0.56 \%$ (94.1\%, 98\%, 85.3\%).

Conclusion: Reduction of proliferative factors (GCSF, FGF, sFas-L) and activation of apoptotic lymphocytes are markers of neutropenia and are associated with the high incidence of sepsis in infants with RDS.

\section{References}

1. Finney SJ, Evans TW: Induction of apoptosis in sepsis: cell suicide may be beneficial. Crit Care Med 2002, 30:261-262.

2. Puchtinskaya MG, Estrin W: The criteria for appointment of a hour-GCSF infants with RDS. Russian J Perinatol Pediatr 2010, 6:34-38.

P34

Haemodynamic and renal effects of clonidine in an ovine model of severe sepsis and septic acute kidney injury

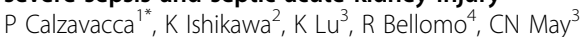

${ }^{1}$ Ospedale Uboldo, Cernusco sul Naviglio, Italy; ${ }^{2}$ Iwate Medical University,

Morioka, Japan; ${ }^{3}$ Melbourne university, Florey Neuroscience Institutes,

Parkville, Australia; ${ }^{4}$ Austin Hospital, Heidelberg, Australia

Critical Care 2012, 16(Suppl 3):P34

Background: In sepsis, the sympathetic nerve activity (SNA) is differentially increased to individual organs [1]. It has been suggested that inhibition of central sympathetic outflow with clonidine would improve outcome in sepsis [2] but the cardiovascular and renal effects of clonidine in sepsis are unknown. Accordingly, we sought to assess the effect of the central $\alpha_{2^{-}}$ agonist clonidine on renal function in an ovine model of severe sepsis.

Methods: Animals had renal and cardiac flow probes implanted to continuously measure the cardiac index $(\mathrm{Cl})$ and renal blood flow (RBF). Mean arterial pressure (MAP) was continuously monitored and hourly 


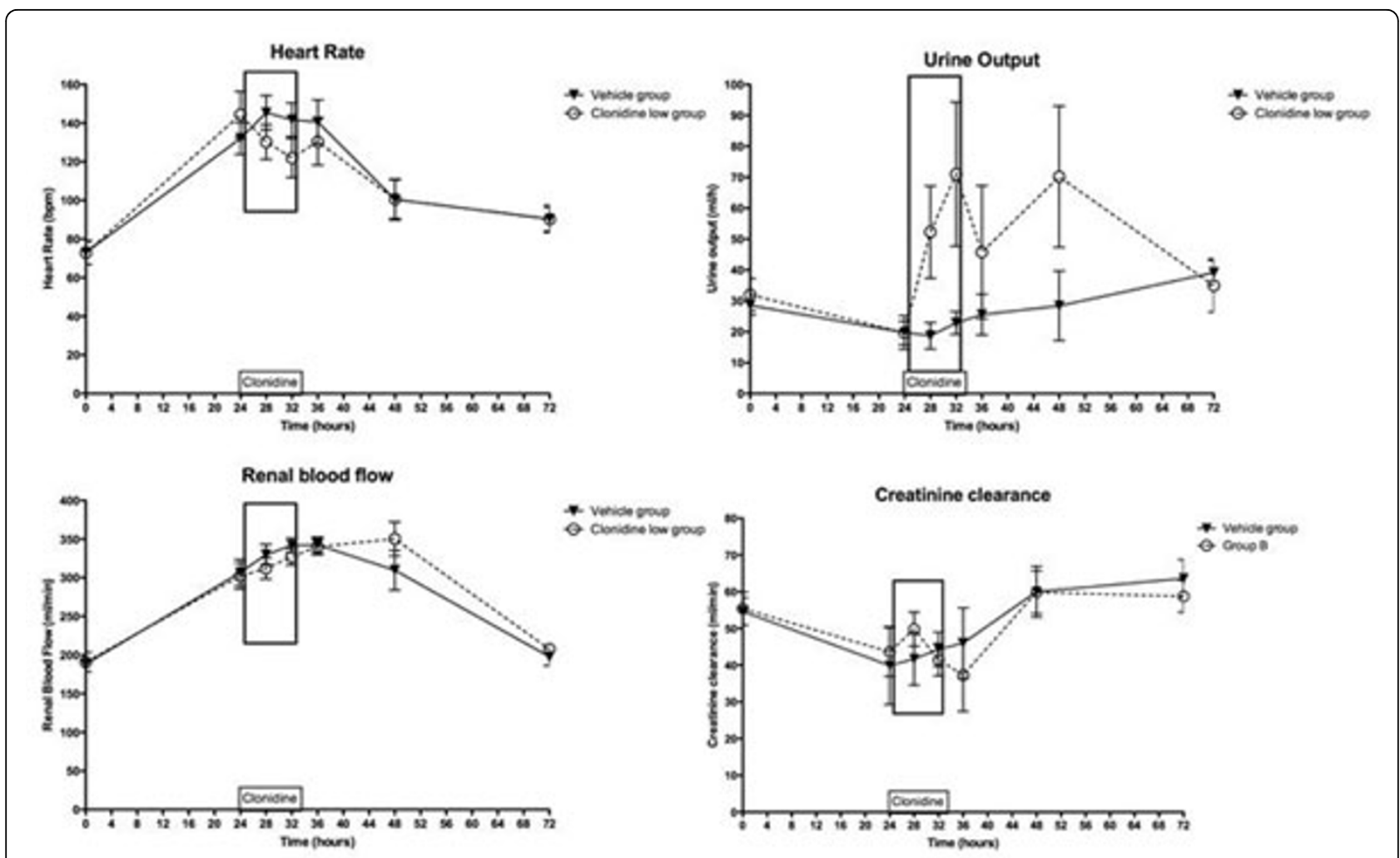

Figure 1(abstract P34) Haemodynamic and renal effects of clonidine in sepsis. Data are mean \pm standard error. Rectangle: 8 hours of clonidine infusion.

urine collection was performed. After the first 24 hours of sepsis every animal was randomly and blindly allocated to receive vehicle or clonidine $0.25 \mu \mathrm{g} / \mathrm{kg} / \mathrm{hour}$ for 8 hours. Animals were followed for further 40 hours during recovery and, at least 2 weeks later, crossed over to the other arm of the study. Two-way repeated-measures ANOVA was performed and $P<0.05$ was considered significant. Data are mean \pm standard error of the average of 4 hours preceding each time point.

Results: Eight animals per group were studied. Two animals per group died during the first experiment, leaving six animals for crossover. Values presented refer to all eight animals. Animals in both groups had similar baseline values and developed a similar hyperdynamic state with $50 \%$ increase in $\mathrm{Cl}, 15 \mathrm{mmHg}$ drop in MAP and threefold increase in arterial lactate. Clonidine (Figure 1) reduced the HR by 23(7) bpm $(P=0.013)$ but did not affect MAP, RBF and lactate levels. Clonidine increased urine output from 23(4) to $71(23) \mathrm{ml} /$ hour $(P=0.004)$, but did not improve creatinine clearance (from $44(6)$ to $42(4) \mathrm{ml} /$ minute).

Conclusion: In experimental hyperdynamic sepsis, treatment with clonidine appears safe, but it does not improve the glomerular filtration rate. References

1. Ramchandra R, Wan L, Hood SG, Frithiof R, Bellomo R, May CN: Septic shock induces distinct changes in sympathetic nerve activity to the heart and kidney in conscious sheep. Am J Physiol Regul Integr Comp Physiol 2009, 297:R1247-R1253.

2. Pichot C, Geloen A, Ghignone M, Quintin L: Alpha-2 agonists to reduce vasopressor requirements in septic shock? Medical Hypotheses 2010, 75:652-656.

P35

Regional perfusion and oxygenation of the kidney in an ovine model of severe sepsis with hypotension and kidney injury

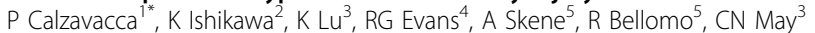

${ }^{1}$ Ospedale Uboldo, Cernusco sul Naviglio, Italy; ${ }^{2}$ Iwate Medical University,

Morioka, Japan; ${ }^{3}$ Melbourne University, Florey Neuroscience Institutes,
Parkville, Australia; ${ }^{4}$ Monash University, Monash, Australia; ${ }^{5}$ Austin Hospital, Heidelberg, Australia

Critical Care 2012, 16(Suppl 3):P35

Background: The pathophysiology of septic acute kidney injury (AKI) is poorly understood. Renal medullary hypoxia has been proposed as a cause of AKl, but the changes in intrarenal oxygenation in hyperdynamic sepsis are unknown. Accordingly, we sought to determine the changes in regional renal perfusion and tissue oxygenation in the cortex and in the medulla in an ovine model of severe sepsis.

Methods: Mean arterial pressure (MAP), cardiac index $(\mathrm{Cl})$ and renal blood flow (RBF) were continuously monitored in conscious sheep. Fibre optic probes (Oxford Optronix) were used to measure tissue perfusion and oxygen partial pressure at 30-second intervals. Arterial and renal venous blood samples were collected for oximetry. After 24 hour of baseline data collection, sepsis was induced with live Escherichia coli infusion for 24 hours. Gentamycin was given to terminate sepsis. The animals were followed for a further 24 hours of recovery. Histology was performed to confirm the position of the catheter tips and to assess tissue viability around the probes. Data are mean ( \pm standard error) of the average of the periods. One-way repeated-measures ANOVA was used and $P<0.05$ considered significant.

Results: Eight animals were studied. All animals developed a hyperdynamic state with a doubling in heart rate and $\mathrm{Cl}$, and a $50 \%$ increase in RBF. MAP decreased by $15 \mathrm{mmHg}$, with a fourfold increase in arterial lactate. Urine output halved, serum creatinine doubled and creatinine clearance decreased by one-third. Two animals died 28 hours after induction of sepsis. Baseline cortical and medullar $\mathrm{pO}_{2}$ were $29.6( \pm 4.3)$ and $29.1( \pm 4.1) \mathrm{mmHg}$, respectively. During sepsis (see Figure 1), cortical perfusion and oxygenation did not change significantly. In contrast, medullary perfusion showed an early trend towards reduction, while medullary tissue $\mathrm{pO}_{2}$ had decreased by $53 \pm 11 \%$ at 24 hours of sepsis $(P<$ $0.05)$. Calculated total renal oxygen consumption did not change significantly (from 33 to $32 \mathrm{ml} \mathrm{O}_{2}$ /minute). 


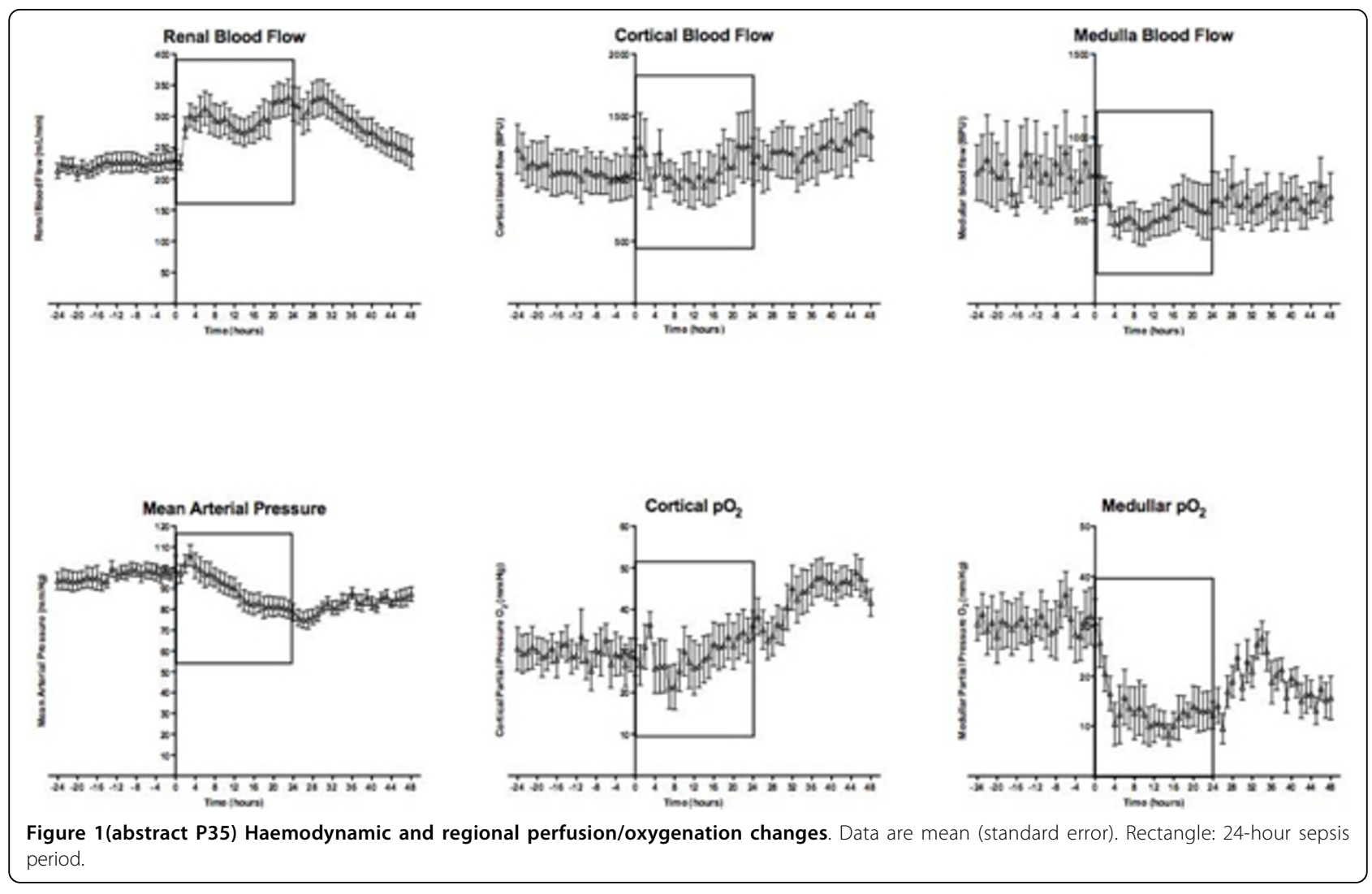

Conclusion: This is the first study to measure intrarenal perfusion and oxygenation in hyperdynamic sepsis. In a conscious large animal model of septic AKI, we showed decreased medullary oxygenation, possibly due to mismatched local perfusion and oxygen consumption.

\section{P36}

Abstract withdrawn

Critical Care 2012, 16(Suppl 3):P36

\section{P37}

Simplified selective decontamination of the digestive tract reduces Gram-negative bloodstream infection and respiratory tract colonization in intensive care

Y Bar-Lavie*, K Hussein, S Hadad, M Barzelay

Rambam Medical Center, Haifa, Israel

Critical Care 2012, 16(Suppl 3):P37

Background: Selective decontamination of the digestive tract (SDD) was shown to reduce acquisition of resistant bacteria and mortality in ICUs. A significant reduction of bacteremia, candidemia, ventilator-associated pneumonia, respiratory tract and rectal colonization have been found in multicenter, randomized studies. SDD was proven to be clinically safe and cost-effective in multiple clinical studies but has not been accepted by the critical care community as a standard of care. Reluctance to use SDD despite proof to the contrary is mostly explained by its perceived potential to raise bacterial resistance to parenteral and enteral antibiotics or rebound infection after their cessation. We studied the effect of a simplified SDD protocol on Gram-negative colonization of the respiratory tract and bloodstream infection.
Methods: During 2011, all adult ICU patients on mechanical ventilation for more than 3 days were included. Enteral medication of neomycin and polymixin-E was given four times daily until gastric tube removal or ICU discharge. Blood, sputum cultures and rectal screening were taken on admission and biweekly until 3 days after ICU discharge. The emergence of multidrug-resistant (MDR) bacteria was investigated and compared with 2010. The in-hospital length of stay and 28-day mortality were compared.

Results: Out of 506 patients, 277 ( $74 \%$ of eligible) received SDD during 2011 , compared with none of 458 patients in 2010. There was a $40 \%$ reduction of positive blood cultures $(P=0.01)$ and a $27.3 \%$ reduction of positive sputum cultures with MDR bacteria $(P=0.04)$. There was a shift from MDR towards sensitive bacteria. This was mostly prominent in the Acinetobacter, Klebsiella and Pseudomonas species. There was no concomitant elevation of other pathogenes as MRSA, VRE, Clostridium or Candida. There was an $18.4 \%$ relative reduction in 28 -day mortality and a 2-day reduction in median hospital length of stay $(P=0.006)$.

Conclusion: The simplified SDD protocol reduced ICU acquisition of Gramnegative resistant infections without a rise in Gram-positives or fungi. The bacterial epidemiology of the unit changed shifting to sensitive bacteria, and the 28-day mortality and hospital length of stay have improved. This study supports the safety and efficacy of this SDD protocol and justifies its further investigation in more ICUs.

\section{P38}

Decreased incidence of SIRS and sepsis by acupuncture in severe multiple traumatic patients via facilitation of vagal activity H Liang*, J Qu

Research Institute of Surgery, Daping Hospital, Daping, Chongqing, China Critical Care 2012, 16(Suppl 3):P38

Background: Acupuncture could increase vagal activity. We hypothesize that acupuncture could activate the cholinergic anti-inflammatory 
pathway, inhibit systemic inflammation, and improve the clinical prognosis in severe multiple trauma patients.

Methods: The design was a prospective, randomized intervention trial. The setting was the Trauma Center, Department of Emergency Medicine, Southwest Hospital, Chongqing, P.R. China. Consecutive multiple trauma patients with an Injury Severity Score (ISS) of more than 16 points meeting the criteria between January 2007 and December 2008 were included. The intervention acupuncture was applied to the ST-36 and PC6 acupoint twice a day until day 7 after injury or sham acupuncture was applied to non-acupoints. One hundred and fifty severe multiple traumatic patients were randomly divided into three groups: acupuncture group (group $A ; n=50$ ), sham acupuncture group (group $B ; n=50$ ), and control group (group $C ; n=50$ ).

Results: Acupuncture at ST-36 and PC-6 acupoints significantly increased the normalized unit of the high-frequency component (HFnu; $P<0.01$ ) and decreased the ratio of low-frequency and high-frequency component (LF/HF; $P<0.05)$, which indicated an increased vagal activity and a tilt of the sympathovagal balance. Serum TNF $\alpha$ and IL- 6 concentrations significantly decreased after stimulation at ST-36 and PC- 6 acupoints $(P<$ 0.01 and $P<0.05$, respectively), but remained unchanged following stimulation at the non-acupoint area. During the whole study period, much lower concentrations of TNF $\alpha$ and IL- 6 were detected in severe traumatic patients with acupuncture intervention in comparison with those in sham acupuncture and control patients $(P<0.05)$. Most importantly, we observed that the incidences of SIRS, and some clinical complications, such as ARDS, sepsis or MOF, and mortality in the acupuncture group were much lower in comparison with the other two groups.

Conclusion: Acupuncture has beneficial effects on modulation of inflammatory response and will improve the clinical outcomes in severe multiple traumatic patients. We suggest that larger cohort studies or multiple center studies are needed to validate whether acupuncture is another effective stimulation pattern to activate the cholinergic antiinflammatory pathway. The detailed mechanisms are worth further investigation.

\section{P39}

A study of Candida biofilms in intensive care patients

R Kaur*, R Goyal, M Singh, P Bhalla, R Kumar

Maulana Azad Medical College, New Delhi, India

Critical Care 2012, 16(Suppl 3):P39

Background: Advances in modern medicine and patient management have seen increased use of prosthetic materials, leading to biofilm formation, and the constituent organisms are different in their growth rates and susceptibility patterns and cause many complications. Candida species are increasingly seen to be important nosocomial pathogens and are major causes of morbidity and mortality in immunocompromised patients of intensive care and postoperative wards. Hence our aims were to isolate and identify specific Candida species associated with the luminal biofilm in endotracheal tubes/i.v. catheters in ICU patients and to study the pathogenicity of Candida species by testing for virulence mechanisms including biofilm formation and antifungal susceptibility.

Methods: One hundred and twenty-five medical/postoperative patients admitted to the ICU for more than 48 hours were studied. Tracheal tubes at the time of extubation and intravenous/other catheters removed from patients and blood samples and other relevant samples from potentially infected sites were collected and processed. Direct microscopy done by $\mathrm{KOH}$ mount and Gram staining and cultures were done on blood agar, brain heart infusion agar, and Sabouraud's Dextose agar with antibiotics and were incubated at 25 and $37^{\circ} \mathrm{C}$. Antifungal susceptibility and virulence testing of Candida species was done by phospholipase, proteinase estimation and adherence assay.

Results: The patients ranged from 13 to 90 years with a male predominance seen in most ages. A total of $86.29 \%$ were admitted for less than 1 week followed by $9.68 \%$ for 1 to 2 weeks. The most common organ system involved was the GIT followed by the CNS, respiratory and multiple organ systems. In total, 356 samples including indwelling devices, blood and urine samples were processed. A total of 253 samples were sterile while fungal isolations were obtained in 103 samples with $51.4 \%$ from indwelling devices and 39.8\% from urine samples. Candida tropicalis (40\%) and Candida albicans (39\%) were the commonest followed by Candida glabrata (11\%), Candida parapsilosis (4\%) and Candida krusei, Candida kefyr and Candida sphaerica (each 2\%). Candida biofilm formation was elicited in 53 indwelling devices. A total of $58.25 \%$ Candida species were resistant to fluconazole. Phospholipase and proteinase activities were seen in $73.8 \%$ and $55.3 \%$ Candida isolates with different species showing a wide range of activities, while 71 Candida isolates showed (4+) adherence activity.

Conclusion: Candida species continue to be important fungal pathogens in the formation of biofilms in ICU patients and knowing drug susceptibility and virulence characteristics will help in reducing associated complications, device control strategies and consequently reducing morbidity, mortality and treatment costs.

\section{P40}

A limited set of molecular biomarkers may provide superior diagnostic outcomes to procalcitonin in sepsis

RB Brandon ${ }^{1 *}$, M Thomas $^{1}$, RA Brandon ${ }^{2}$, D Venter ${ }^{3}$, J Presneill ${ }^{3}$, J Lipman ${ }^{4}$, J Morgan ${ }^{3}$, B Venkatesh ${ }^{5}$, J Sackier ${ }^{2}$, A Sutherland

${ }^{1}$ Immunexpress Pty Ltd, Brisbane, Australia; ${ }^{2}$ Immunexpress Inc., Seattle, WA, USA; ${ }^{3}$ Mater Health Services, Brisbane, Australia; ${ }^{4}$ The University of Queensland and Royal Brisbane \& Women's Hospital, Brisbane, Australia;

${ }^{5}$ Princess Alexandra Hospital, Brisbane, Australia

Critical Care 2012, 16(Suppl 3):P40

Background: Differentiating the systemic inflammatory response syndrome (SIRS) from sepsis is very important to clinicians. Procalcitonin (PCT) has been studied extensively as a marker of sepsis; however, its clinical utility remains uncertain [1]. Alternative approaches involving analysis of circulating biomarkers using gene expression (GE) show promise [2]. The primary objective of this study was to compare the diagnostic performance of a GE biomarker set, SeptiCyte ${ }^{\oplus}$ Triage, with PCT in a mixed patient population.

Methods: The dataset was derived from two clinical trials conducted across four tertiary care settings in Australia between 2008 and 2011 (ACTRN12610000465055). Critical care patients ( $n=87$ ) were enrolled if they fulfilled the 1992 Consensus Statement [3] for sepsis, severe sepsis or septic shock. Postsurgical patients were included as an infection-negative systemic inflammatory cohort $(n=31)$, with 71 healthy controls $(\mathrm{HC})$ for comparison. Blood samples were collected within 24 hours of the surgical procedure or upon admission to ICU for sepsis patients. PCT was measured using a commercially available assay kit (Brahms PCT) and GE assessed using Affymetrix GeneChip Human Exon 1.0 ST arrays, where a set of biomarkers were identified a priori. A Support Vector Machine algorithm was used to calibrate the molecular biomarkers with respect to specific clinical groups. Bootstrap and permutation tests were performed on the difference in area under the receiver operating characteristic curves (AUC $\mathrm{ROC})$ for PCT and crossvalidated posterior probabilities.

Results: The SeptiCyte ${ }^{\oplus}$ Triage molecular biomarker set was significantly better than PCT at differentiating non-infectious systemic inflammation from all sepsis groups with an AUC ROC of $99.1 \%$ versus $90.8 \%(95 \% \mathrm{Cl}$ of difference $=4 \%, 16 \%$ ), respectively. The differentiating ability of PCT and the SeptiCyte ${ }^{\oplus}$ Triage biomarker set is demonstrated in Figure 1. The 99.1\% AUC ROC of the biomarker set could be maintained when using a set of less than 10 genes, important for transferring the biomarkers to a rapid assay format. As a quality step, a classifier was developed that differentiated $\mathrm{HC}$ from sepsis with an AUC ROC of $100 \%$.

Conclusion: In comparison with the diagnostic performance of PCT, a limited set $(<10)$ of molecular biomarkers potentially has a significantly better sensitivity and specificity profile for the detection of sepsis within a mixed systemic inflammatory patient group.

References

1. Tang B, Eslick GD, Craig JC, McLean AS: Accuracy of procalcitonin for sepsis diagnosis in critically ill patients: systemic review and metaanalysis. Lancet Infect Dis 2007, 7:210-217.

2. Sutherland A, Thomas M, Brandon RA, Brandon RB, Lipman J, Tang B, McLean A, Pascoe R, Price G, Nguyen T, et al: Development and validation of a novel molecular biomarker diagnostic test for the early detection of sepsis. Crit Care 2011, 15:R149.

3. American College of Chest Physicians/Society of Critical Care Medicine: Crit Care Med 1992, 20:864-874. 


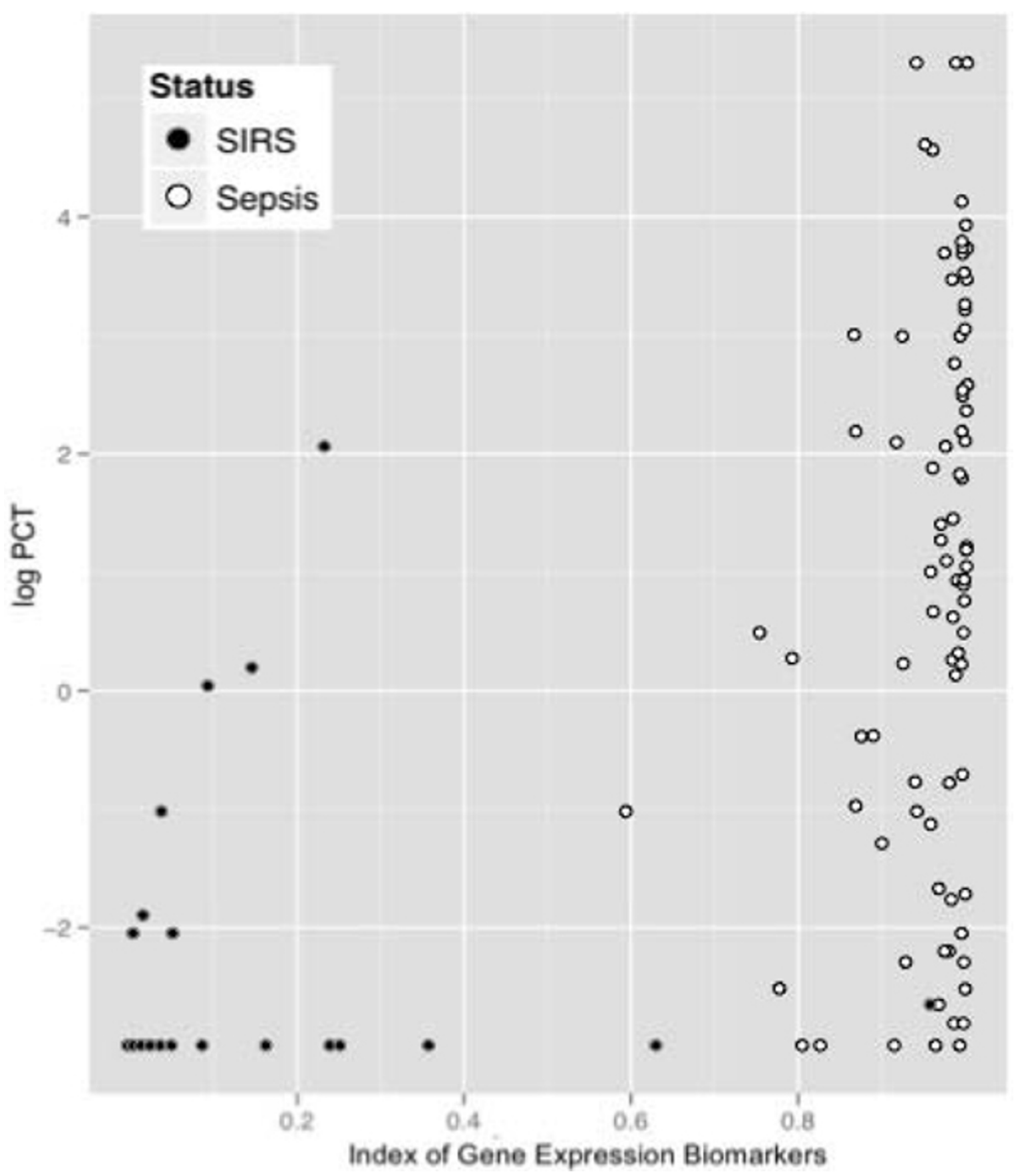

Figure 1(abstract P40) Plot of log PCT (y axis) and index of SeptiCyte ${ }^{\oplus}$ Triage gene expression biomarkers ( $x$ axis) for 31 SIRS (black) and 87 sepsis (white) patients demonstrating the ability of each technology to differentiate these conditions.

P41

Monocytic and neutrophilic CD11b and CD64 in severe sepsis

J Jämsä ${ }^{*}$, V Huotari ${ }^{2}$, ER Savolainen ${ }^{2}$, H Syrjälä², T Ala-Kokko ${ }^{2}$

${ }^{1}$ University of Oulu, Finland; ${ }^{2}$ Oulu University Hospital, Oulu, Finland

Critical Care 2012, 16(Suppl 3):P41

Background: Leukocyte immunophenotyping could improve sepsis diagnostics [1,2]. Our hypothesis was that monocytic and neutrophilic CD11b and CD64 antigen fluorescence intensities differ between severe sepsis, non-inflammatory ICU patients and nonseptic inflammation (offpump coronary artery bypass (OPCABG)).

Methods: Monocytic and neutrophilic CD11b and CD64 expressions were analyzed from 27 patients with severe sepsis, seven OPCABG patients and from eight ICU patients who did not fulfill any SIRS criteria. Blood samples were collected within 48 hours from the beginning of severe sepsis or in non-SIRS patients from ICU admission and two consecutive days (D0, D1, D2). From surgical patients, the first samples were taken on the day of surgery before the skin incision and two consecutive days (D0, D1, D2). In addition 10 healthy individuals served as controls. Samples 


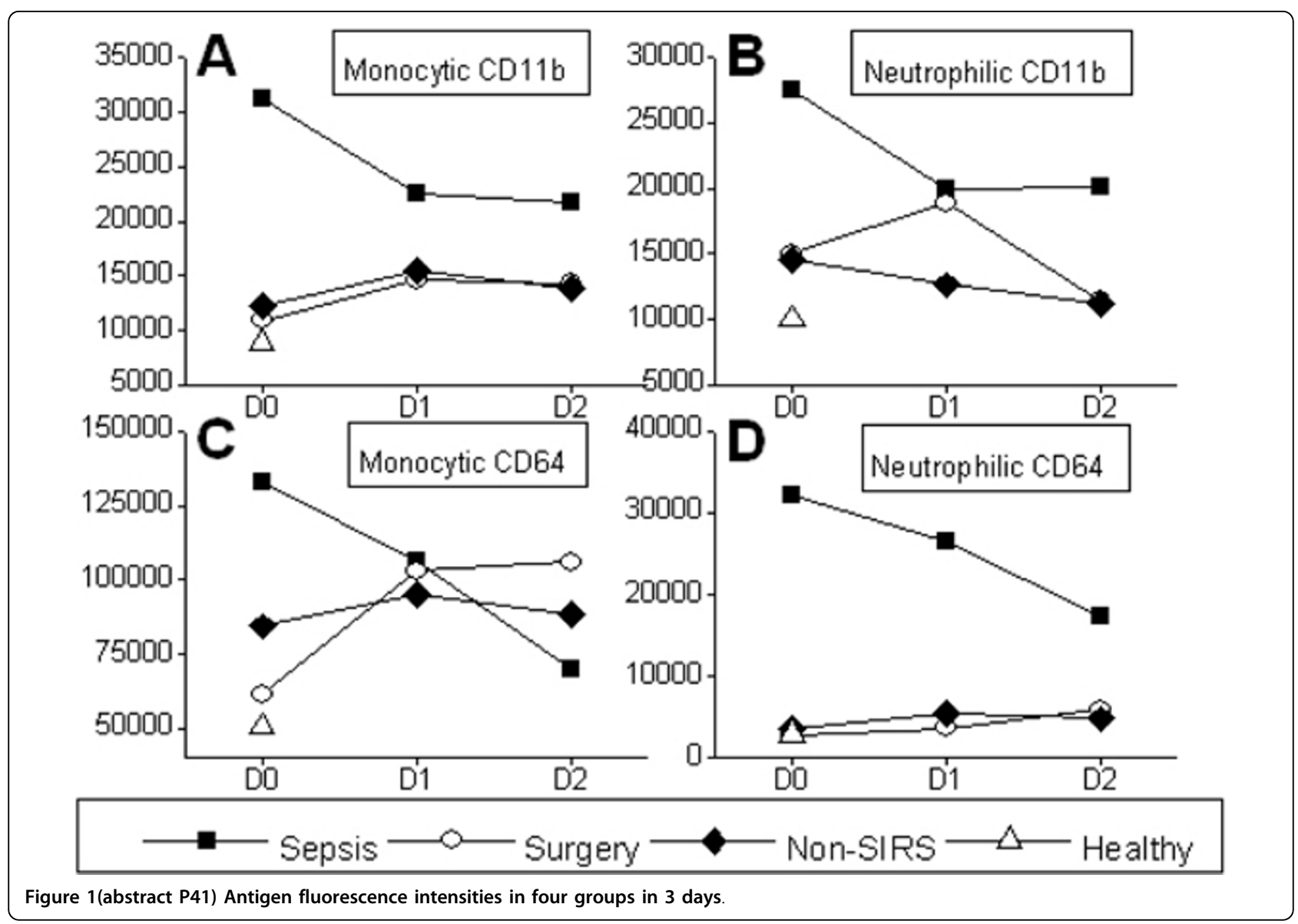

were collected, processed and analyzed using flow cytometry as previously described [3].

Results: The maximum fluorescence intensities of monocytic and neutrophilic CD11b and CD64 were highest in septic patients compared with the other groups $(P<0.05)$ (Figure 1$)$. In severe sepsis, fluorescence intensities decreased over time $(P<0.05)$. In OPCABG the fluorescence intensities of other antigens increased from D0 to D1 except neutrophilic CD11b $(P<0.05)$. The intensities of other antigens except neutrophilic CD64 were lower in the healthy than in all the other groups $(P<0.05)$. Neutrophilic CD64, as well as other antigens, were lower in healthy controls compared with severe sepsis at all time points $(P<0.05)$.

Conclusion: Based on this study, monocytic CD11b and neutrophilic CD64 could be helpful in distinguishing severe sepsis from nonseptic inflammation and healthy controls.

References

1. Hoffmann J: Neutrophil CD64: A diagnostic marker for infection and sepsis. Clin Chem Lab Med 2009, 47:903-916.

2. Nuutila J, Jalava-Karvinen P, Hohenthal U, Laitinen I, Kotilainen P, Rajamaki A, Nikoskelainen J, Lilius E: CRP/CD11b ratio: a novel parameter for detecting gram-positive sepsis. Hum Immunol 2009, 70:237-243.

3. Jamsa J, Huotari V, Savolainen E-R, Syrjala H, Ala-Kokko T: Analysis of the temperature affects on leukocyte surface antigen expression. J Clin Lab Anal 2011, 25:118-125.

P42

Clinical evaluation of the Magicplex Sepsis Real-time Test (Seegene) to detect Candida DNA in pediatric patients

J Serra*, E Rosello, C Figueras, M Pujol, Y Peña, P Céspedes, JL Dapena,

C Díaz-Heredia, MG Codina, A Andreu

Hospital Vall d'hebron, Barcelona, Spain

Critical Care 2012, 16(Suppl 3):P42
Background: Invasive candidiasis is an important cause of morbidity and mortality among hospitalized patients. A rapid diagnosis is extremely important for a successful outcome. Standard diagnostic methods (culture and direct examination) often lack a satisfying sensitivity and require a long growth period. The aim of the study was to assess the performance of a new real-time PCR multiplex technique to rapidly detect Candida DNA in clinical samples compared with culture-based methods.

Methods: Between November 2011 and May 2012, 142 blood samples from 71 pediatric critical or hematological patients were studied both by culture and PCR. The Magicplex Sepsis Real-time Test (Magicplex ${ }^{\mathrm{TM}}$ system; Seegene) was used to detect Candida albicans, Candida parapsilosis, Candida glabrata, Candida tropicalis and Candida krusei. Prior to DNA extraction (EZ1 automatic system; Qiagen) at least $1 \mathrm{ml}$ of blood sample was inoculated into a VersaTREK REDOX1 bottle, which was incubated at $37^{\circ} \mathrm{C}$ for $\geq 3$ hours. The PCR technique was performed according to the manufacturer's instructions. Culture-based methods were conduced simultaneously in all specimens using the BacT/ALERT system (BioMérieux). Results: A total of 131 of the 142 samples were both culture and PCR negative. Nine blood samples (belonging to eight patients) were positive by PCR, two of them were also positive by culture and the remaining seven were not; two of the unfitting samples $(28.5 \%)$ belonged to patients with other multiple positive cultures for Candida spp. (urine and/or catheter devices); the others belonged to patients with high clinical suspicion and risk factors for invasive candidiasis. On the other hand, two culture-proven and PCR-negative samples were considered false negative results. Blood culture allowed the diagnosis of four patients (two of them were also positive for $\mathrm{PCR}$ ), while the evaluated $\mathrm{PCR}$ technique permitted the detection of six additional cases (one of them with two positive samples).

Conclusion: In this study the evaluated PCR system provides a rapid technique for Candida detection. It can be considered an effective diagnostic tool for diagnosing invasive candidiasis along with conventional cultures. 
P43

Procalcitonin, IL-10 and SCD25 as diagnostic and prognostic markers in critically ill patients

A Focà ${ }^{1 *}$, MC Liberto ${ }^{1}$, L Rametti ${ }^{1}$, A Giancotti ${ }^{1}$, N Marascio $^{1}$, A Quirino ${ }^{1}$,

S Caroleo ${ }^{2}$, A Renzulli $^{3}$, G Matera $^{1}$

${ }^{1}$ University of Catanzaro Institute of Microbiology, Catanzaro, Italy; ${ }^{2}$ University of Catanzaro Anesthesiology Unit, Catanzaro, Italy; ${ }^{3}$ University of Catanzaro Cardiac Surgery Unit, Catanzaro, Italy

Critical Care 2012, 16(Suppl 3):P43

Background: Diagnostic and prognostic markers are of outmost importance in the critical patient referred to the ICU. The aim of the present study was the evaluation of procalcitonin (PCT), IL-10 and soluble CD25 (sCD25) as potential markers in the diagnosis and prognosis of cohorts of critical patients evaluated following a prospective design in a tertiary-center university hospital.

Methods: We studied 52 consecutive SIRS patients with suspected sepsis that were firstly stratified based on culture results (culture-positive and culture-negative), then subsequently divided into survivors and nonsurvivors. Venous blood samples were obtained from each patient within 6 hours of hospital/ICU admission (T-0) and at the end of first week of hospital/ICU stay (T-1). Serum samples were collected and used for simultaneous determination of IL-10 and sCD25, using a cytokine biochip array on the Evidence Investigator analyser (Randox Laboratories Ltd, Crumlin, UK), while PCT was assayed by an enzyme-linked fluorescent assay (VIDAS BRAHMS PCT; bioMerieux, France). Statistically significant differences between groups were established by the Mann-Whitney $U$ test. The receiver-operating characteristic (ROC) curve was used to evaluate the diagnostic accuracy (defined by the area under the ROC curve (AUROCC)) of the analyzed cytokines and to determine the sensitivity and specificity a selected cutoff values. The statistical analyses were performed using SPSS 14.0 software (SPSS, Chicago, IL, USA).

Results: PCT, IL-10 and SCD25 were significantly $(P<0.05)$ increased in infectious versus non-infectious SIRS patients at hospital admission (T-0) and after 1 week of hospital stay (T-1). Diagnostic accuracy of PCT, IL-10 and SCD25 was evaluated by AUROCC and exhibited a significance index of $0.0001,0.0021$ and 0.0095 respectively at T-0, while at $\mathrm{T}-1$ the $P$ values were $0.0011,0.0016$ and 0.0201 respectively. Regarding prognostic markers, PCT showed a prognostic significance only at T-1 $(P=0.04)$. However when IL-10 and SCD25 values from survivors were compared with those from nonsurvivors, significant differences were found for the former and the latter marker with $P=0.0014$ and $P=0.014$ respectively at $T-0$, as well as with $P=0.0002$ and $P=0.014$ respectively at T-1.

Conclusion: In our study, well-known PCT and IL-10 markers and novel sCD25 significantly contributed to diagnosis and prognosis of SIRS and septic patients.

\section{P44}

Ninjurin 1 contributes to TLR-induced inflammation in endothelial cells C Jennewein*, K Zacharowski

Hospital of the Goethe-University Frankfurt, Clinic of Anaesthesiology,

Intensive Care and Pain Therapy, Frankfurt, Germany

Critical Care 2012, 16(Suppl 3):P44

Background: Nerve injury induced protein 1 (Ninjurin 1 (Ninj1)) was first identified in Schwann cells and neurons contributing to cell adhesion and nerve regeneration. Recently, the role of Ninj1 has been linked to inflammatory processes in the central nervous system where functional repression reduced leukocyte infiltration and clinical disease activity during experimental autoimmune encephalomyelitis in mice [1]. But Ninj1 is also expressed outside the nervous system in various organs such as the liver and kidney as well as on leukocytes $[2,3]$. Therefore, we hypothesized that Ninj 1 contributes to inflammation in general; that is, also outside the nervous system, with special interest in the pathogenesis of sepsis.

Methods: Ninj1 was repressed by transfecting HMEC-1 cells, a human dermal microvascular endothelial cell line with siRNA targeting Ninj1 (siNinj1) or a negative control (siC). Subsequently, cells were stimulated with $100 \mathrm{ng} / \mathrm{ml}$ LPS (TLR4 agonist), $3 \mu \mathrm{g} / \mathrm{ml}$ LTA (TLR2 agonist) or $100 \mathrm{n} / \mathrm{ml}$ poly(l: C) (TLR3 agonist) for 3 hours. The inflammatory response was analyzed by real-time PCR. In addition, transmigration of neutrophils across a HMEC-1 monolayer was measured using transwell plates (pore size $3 \mu \mathrm{m}$ ).

Results: Repression of Ninj1 by siRNA reduced Ninj1 mRNA expression in HMEC about 90\% (Figure 1A). Reduced Ninj1 expression decreased neutrophil migration to $62.5 \%$ (Figure $1 \mathrm{~B}$ ) and TLR signaling. In detail knockdown of Ninj1 significantly reduced TLR-2 and TLR-4 triggered expression of ICAM-1 and IL-6 (Figure 1C,D) while poly(I:C)-induced

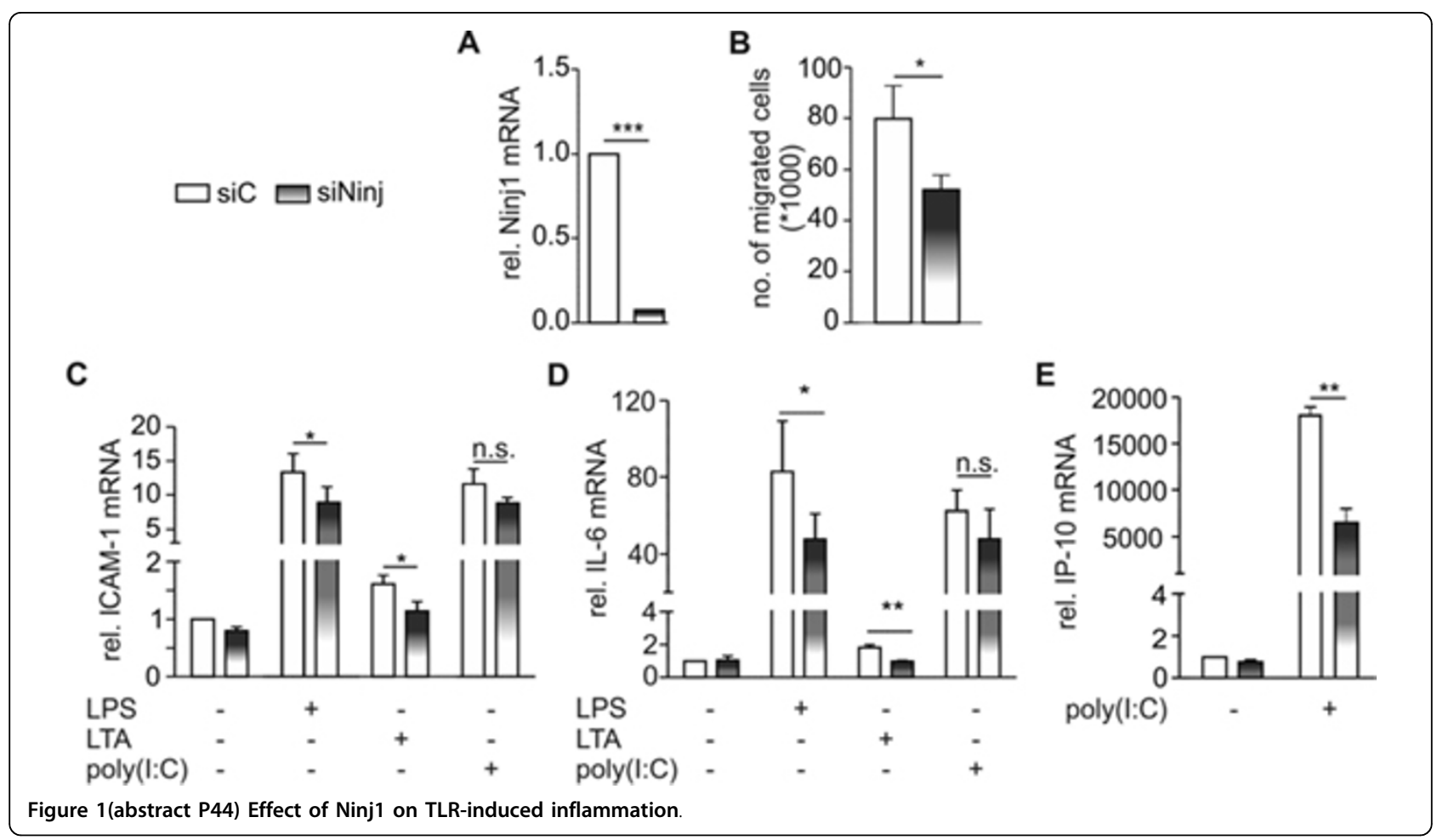


expression was only slightly reduced. To analyze a more specific TLR-3 target, we measured IP-10 mRNA expression, which was also significantly reduced in siNinj1-transfected cells (Figure 1E).

Conclusion: Our in vitro data strongly indicated that Ninj1 is involved in regulation of TLR signaling and therewith contributes to inflammation. In vivo experiments will clarify its impact on systemic inflammation. References

1. Ifergan I, Kebir H, Terouz S, Alvarez Jl, Lecuyer MA, Gendron S, Bourbonniere L, Dunay IR, Bouthillier A, Moumdjian R, et al: Role of ninjurin-1 in the migration of myeloid cells to central nervous system inflammatory lesions. Ann Neurol 2011, 70:751-763.

2. Araki T, Zimonjic DB, Popescu NC, Milbrandt J: Mechanism of homophilic binding mediated by ninjurin, a novel widely expressed adhesion molecule. J Biol Chem 1997, 272:21373-21380.

3. Araki T, Milbrandt J: Ninjurin, a novel adhesion molecule, is induced by nerve injury and promotes axonal growth. Neuron 1996, 17:353-361.

\section{P45}

Effect of Calotropis procera latex extracts on the hypothalamic TNF $\alpha$ and $\mathrm{PGE}_{2}$ levels in the rat model of yeast-induced pyrexia

B Guruprasad*, P Chaudhary, VL Kumar

All India Institute of Medical Sciences, New Delhi, India

Critical Care 2012, 16(Suppl 3):P45

Background: Sepsis, a common cause of morbidity and mortality in critically ill patients, is associated with systemic inflammatory response syndrome due to upregulation of cyclooxygenase-2 and increase in the levels of $P G_{2}$. It is also associated with increase in the levels of proinflammatory cytokines like TNF $\alpha$ and IL- $1 \beta$. Calotropis procera is a plant that grows in the wild producing latex. The aqueous and methanolic extracts of dried latex of this plant (AqDL and $\mathrm{MeDL}$ ) and proteins isolated from the fresh latex (LP) have shown anti-inflammatory and anti-arthritic properties. AqDL and MeDL are orally effective, LP is effective parenterally. The current study was designed to evaluate the efficacy of these extracts against yeast-induced pyrexia and the levels of TNF $\alpha$ and PGE 2 in the hypothalamus of rats.

Methods: Pyrexia was induced in rats by subcutaneous injection of yeast in the nape of the neck and the rectal temperature was measured at 0 hours (basal temperature), 3 hours and 6 hours. Rats were divided into groups $(n=6)$ and were treated with AqDL and MeDL given orally and LP given intravenously at 6 hours. Group I: NC (normal control); Group II: YC (yeast control); Group III: AqDL ( $200 \mathrm{mg} / \mathrm{kg})$ ) Group IV: AqDL (400 mg/kg); Group V: MeDL (100 mg/kg); Group VI: MeDL (250 mg/kg); Group VII: LP (5 mg/kg); Group VIII: LP (25 mg/kg); Group IX: paracetamol (PCM $100 \mathrm{mg} / \mathrm{kg}$ ). Rectal temperature was measured hourly until 9 hours. The levels of TNF $\alpha$ and $\mathrm{PGE}_{2}$ were measured in the excised hypothalamus region of the brain using ELISA kits.

Results: Subcutaneous injection of yeast produced a marked increase in rectal temperature of rats with a maximum effect at 6 hours $\left(101.17^{\circ} \mathrm{C}\right)$. Like paracetamol, treatment of rats with $\mathrm{AqDL}$ and MeDL produced a significant decrease in body temperature from $101.17^{\circ} \mathrm{C}$ at 6 hours to $97.9^{\circ} \mathrm{C}$ and $98.2^{\circ} \mathrm{C}$ at 9 hours respectively at higher doses and their effect was dose dependent while LP was found to be ineffective. The present study shows that treatment with yeast increased the tissue levels of TNF $\alpha(23.78 \mathrm{pg} / \mathrm{mg})$ and $\mathrm{PGE}_{2}(66.48 \mathrm{pg} / \mathrm{mg}$ ) as compared with the NC group (16.31 and 41.35 respectively). All of the fractions lowered the hypothalamic TNF $\alpha$ level while a marked reduction in $\mathrm{PGE}_{2}$ levels was observed with orally effective fractions, namely AqDL and MeDL.

Conclusion: Our results demonstrate that the orally administered fractions of latex of $C$. procera are effective in attenuating yeast-induced pyrexia and this effect is mediated through reduction in the levels of $\mathrm{PGE}_{2}$.

\section{P46}

Regulation of sepsis-induced IFN $\gamma$ upon natural killer cell or natural killer T cell depletion in vivo

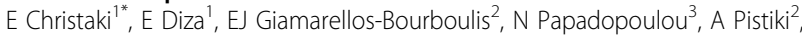
D Droggiti', A Machova ${ }^{3}$, M Georgitsi $i^{2}$, L Lambrelli', G Karkavelas', A lliadis', N Malisiovas' ${ }^{1}$, P Nikolaidis' ${ }^{1}$ SM Opal ${ }^{5}$

${ }^{1}$ Aristotle University of Thessaloniki Medical School, Thessaloniki, Greece;

${ }^{2}$ University of Athens Medical School, Athens, Greece; ${ }^{3}$ University of Cologne,
Institute for Medical Microbiology Immunology and Hygiene, Cologne, Germany; ${ }^{4}$ University of Macedonia, Thessaloniki, Greece; ${ }^{5}$ Alpert School of Medicine of Brown University, Providence, RI, USA

Critical Care 2012, 16(Suppl 3):P46

Background: Natural killer (NK) and natural killer T (NKT) cells play a key role in bacterial infection and sepsis since they contribute to the bridging of innate and acquired immune responses. We have previously shown that in vivo depletion of these cell populations in a murine pneumococcal pneumonia sepsis model affected mortality.

Methods: Four groups of C57BL/6 mice ( $n=5$ to 15 mice/group) were infected intratracheally with $5 \times 10^{5}$ CFU Streptococcus pneumoniae. Twenty-four hours prior to bacterial inoculation, NK cell depletion was achieved by intravenous (i.v.) administration of anti-asialoGM1 rabbit polyclonal antibody in one group (NK ${ }^{\mathrm{DEPL}}$ ), or anti-CD1d monoclonal antibody, clone 1B1 was given for NKT cell depletion in a second group $\left(\mathrm{NKT}^{\mathrm{DEPL}}\right)$. The control group received equal volume of isotype antibody control i.v. (C) and a fourth group received sham intratracheal installation of normal saline (S). All animals were euthanized 48 hours post infection. Serum and tissue samples were analyzed for bacterial colony counts, cytokine levels, splenocyte apoptosis rates and cell population analysis by flow cytometry. In parallel, specific miRNA expression analyses in splenocytes and lung histologic examination were also performed. Comparisons of numeric data between groups were made using the oneway ANOVA test for multiple groups.

Results: We found that upon NK cell depletion there was a significant increase in the spleen NKT $\left(\mathrm{CD}^{+} / \mathrm{CD} 1 \mathrm{~d}^{+}\right)$cell population compared with $\mathrm{NKT}^{\mathrm{DEPL}}, C$ and $S(P=0.014, P=0.021$ and $P=0.033$, respectively). Interestingly, upon NKT cell depletion, spleen NK $\left(\mathrm{CD}^{-} / \mathrm{NK} 1.1^{+}\right)$cells increased significantly compared with NK ${ }^{\mathrm{DEPL}}, C$ and $\mathrm{S}(P<0.0001, P<$ 0.0001 and $P=0.001$, respectively). NKT depletion led to decreased lymphocyte apoptosis compared with $C(P=0.035)$, higher bacterial load in the lung compared with $C$ and $\operatorname{NK}^{\mathrm{DEPL}}(P=0.014$ and $P=0.022$ respectively) and in the liver compared with $C(P=0.012)$. In addition, serum levels of IFN $\gamma$ were significantly increased and splenocytes from NKT depleted animals, incubated ex vivo in the presence or absence of IL-2, produced more IFN $\gamma$ in comparison with all other groups. Furthermore, splenocyte miRNA analysis showed that miR-200c and miR-29a were downregulated, while miR-125a-5p was upregulated, in the NKT depleted animals compared with all other groups.

Conclusion: For the first time we have shown that NKT cell depletion resulted in an increase in spleen NK $\left(\mathrm{CD}^{-} / \mathrm{NK}^{-1} 1^{+}\right)$cells and a higher IFN $\gamma$ production, which were associated with specific changes in splenocyte miRNA expression.

\section{P47}

Pattern recognition receptors as key players in adrenal gland dysfunction during sepsis

THN Tran ${ }^{1 *}$, W Kanczkowski ${ }^{2}$, V Hoesker ${ }^{1}$, SR Bornstein ${ }^{2}$, K Zacharowski $^{1}$

'Hospital of the Goethe-University Frankfurt, Clinic of Anaesthesiology Intensive Care Medicine and Pain Therapy, Frankfurt am Main, Germany;

${ }^{2}$ Technical University Dresden, Germany

Critical Care 2012, 16(Suppl 3):P47

Background: Undergoing systemic inflammation, the innate immune system releases excessive proinflammatory mediators, which finally can lead to organ failure. Pattern recognition receptors (PRRs), such as Toll-like receptors (TLRs) and NOD-like receptors (NLRs), form the interface between bacterial and viral toxins and innate immunity. During sepsis, patients with diagnosed adrenal gland insufficiency are at high risk of developing a multiorgan dysfunction syndrome, which dramatically increases the risk of mortality. To date, little is known about the mechanisms leading to adrenal dysfunction under septic conditions. Here, we investigated the sepsisrelated activation of the PRRs, cell inflammation, and apoptosis within adrenal glands.

Methods: Two sepsis models were performed: the polymicrobial sepsis model (caecal ligation and puncture (CLP)) and the LTA-induced intoxication model. All experiments received institutional approval by the Regierungspräsidium Darmstadt. CLP was performed as previously described [1], wherein one-third of the caecum was ligated and punctured with a 20-gauge needle. For LTA-induced systemic inflammation, TLR2 
knockout $\left(\right.$ TLR2 $\left.^{-1}\right)$ and WT mice were injected intraperitoneally with pure LTA (pLTA; $1 \mathrm{mg} / \mathrm{kg}$ ) or PBS for 2 hours. To detect potential direct adrenal dysfunction, mice were additionally injected with adrenocorticotropic hormone (ACTH; $100 \mu \mathrm{g} / \mathrm{kg}) 1$ hour after pLTA or PBS. Adrenals and plasma samples were taken. Gene expressions in the adrenals (rt-PCR), cytokine release (multiplex assay), and the apoptosis rate (TUNEL assay) within the adrenals were determined.

Results: In both models, adrenals showed increased mRNA expression of TLR2 and TLR4, various NLRs, cytokines as well as inflammasome components, NADPH oxidase subunits, and nitric oxide synthases (data not shown). In WT mice, ACTH alone had no effect on inflammation, while PLTA or pLTA/ACTH administration showed increased levels of the cytokines IL-1 $\beta$, IL-6, and TNF $\alpha$. TLR2 ${ }^{-1-}$ mice indicated no response as expected (Figure 1, left). Interestingly, surviving CLP mice showed no inflammatory adrenal response, whereas nonsurvivors had elevated cytokine levels (Figure 1, right). Additionally, we identified a marked increase in apoptosis of both chromaffin and steroid-producing cells in adrenal glands obtained from mice with sepsis as compared with their controls (Figure 2).

Conclusion: Taken together, sepsis-induced activation of the PRRs may contribute to adrenal impairment by enhancing tissue inflammation, oxidative stress and culminate in cellular apoptosis, while mortality seems to be associated with adrenal inflammation.

\section{Reference}

1. Rittirsch D, Huber-Lang MS, Flierl MA, Ward PA: Immunodesign of experimental sepsis by cecal ligation and puncture. Nat Protoc 2009, 4:31-36.

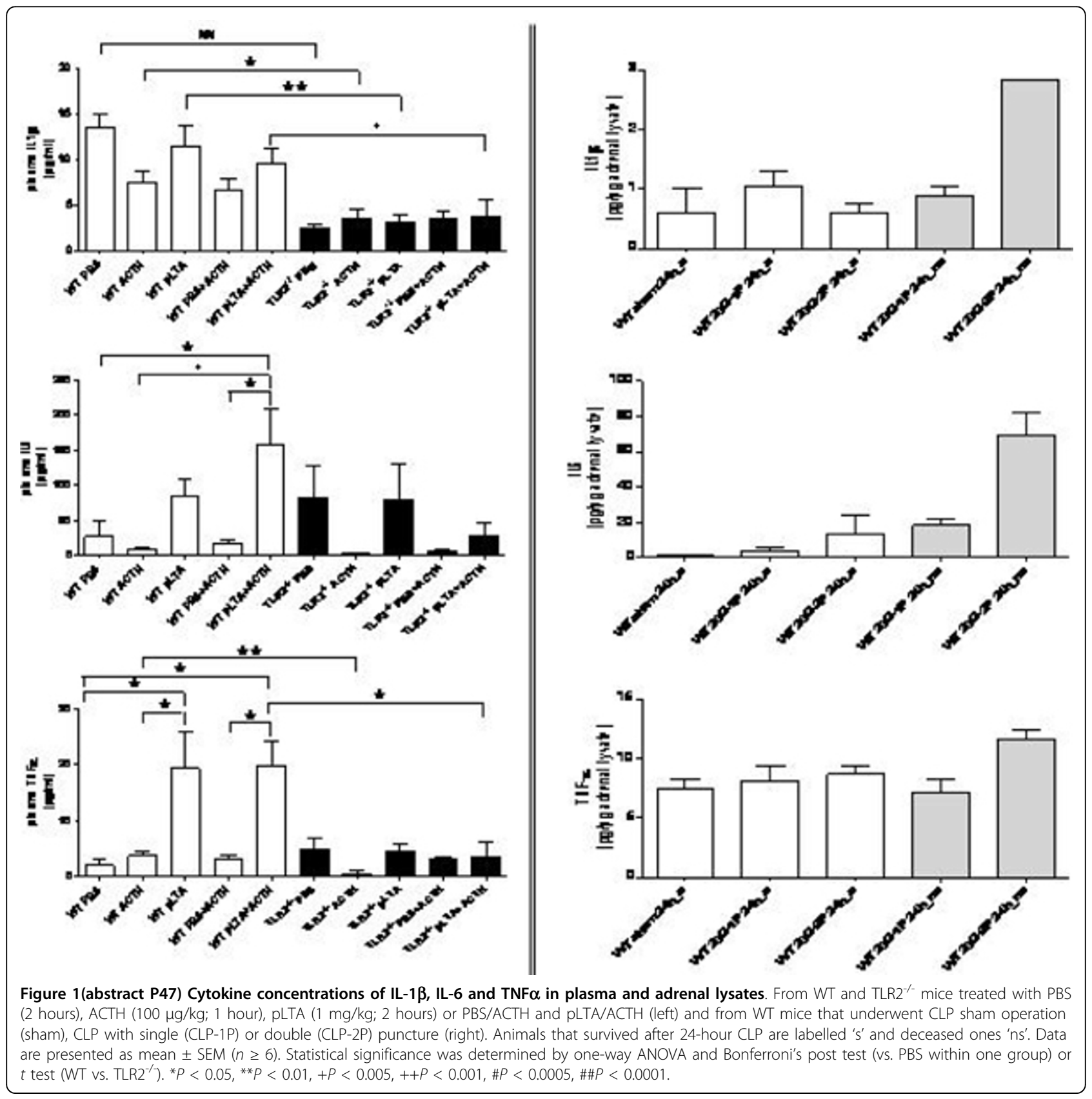




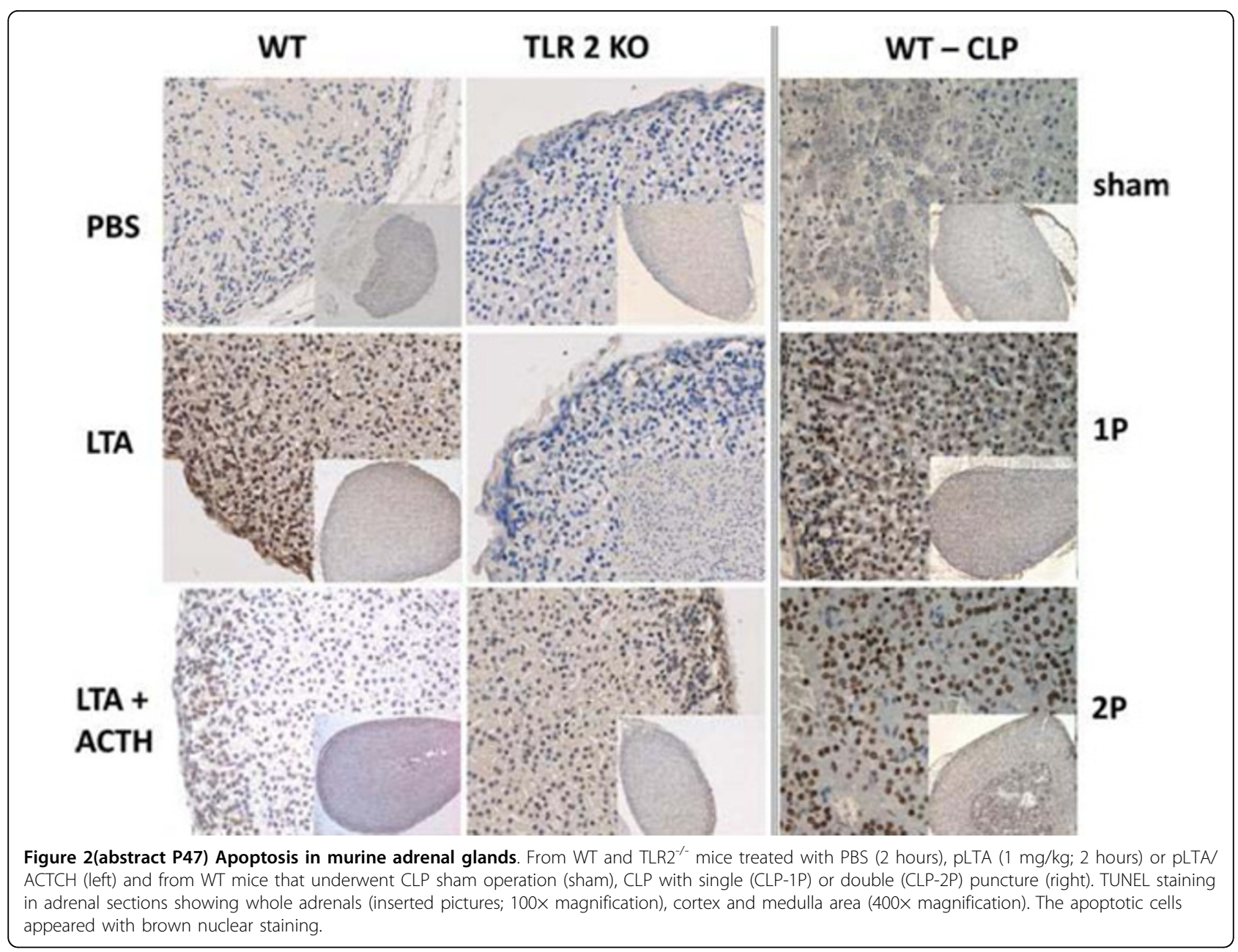

\section{P48}

Effects of a TREM-like transcript-1 derived peptide during septic shock in pigs

S Gibot ${ }^{1 *}$, A Boufenzer $^{2}$, Y Bouazza ${ }^{2}$, F Groubatch ${ }^{3}$, C Alauzet $^{4}$, D Barraud ${ }^{1}$, PE Bollaert ${ }^{1}, \mathrm{P}$ Leroy $^{5}, \mathrm{~N} \operatorname{Tran}^{3}, \mathrm{M}^{2}$ Derive $^{2}$

${ }^{1} \mathrm{CHU}$ Nancy, Nancy, France; ${ }^{2}$ Nancy University, Groupe CHOC - Inserm U961, Nancy, France; ${ }^{3}$ Nancy University, School of Surgery - U961, Nancy, France;

${ }^{4}$ Nancy University, EA4369, Nancy, France; ${ }^{5}$ Nancy University, EA3452

CITHEFOR, Nancy, France

Critical Care 2012, 16(Suppl 3):P48

Background: Triggering receptor expressed on myeloid cells-1 (TREM-1) is expressed on innate immune cells and plays a crucial role during the onset of sepsis by amplifying the host immune response. TREM-like transcript-1 (TLT-1) belongs to the TREM family and is selectively expressed on activated platelets. We recently showed that TLT-1 and a TLT-1-derived peptide (LR12) exhibit anti-inflammatory properties by dampening TREM-1 signalling and thus behave as naturally occurring TREM-1 inhibitors [1]. We also, however, demonstrated that the same peptide modulates in vivo the inflammatory cascade triggered by infection, thus inhibiting hyper-responsiveness, organ damage and death during sepsis in mice. As mouse models of septic shock are far from recapitulating the human physiology, we investigated the effects of LR12 during peritonitis in adult mini-pigs. Here we show that sepsis-induced cardiovascular dysfunction and organ failure was prevented by LR12 administration. The objective was to determine the effects of a TLT-1 derived peptide (LR12) administration during septic shock in pigs (13 adult male mini-pigs).

Methods: Two hours after induction of a fecal peritonitis, anesthetized and mechanically ventilated mini-pigs were randomized to receive LR12 $(n=6)$ or its vehicle alone (normal saline, $n=5)$. Two animals were operated and instrumented without the induction of peritonitis and served as controls (sham). Resuscitation was achieved using hydroxyethyl starch (up to $20 \mathrm{ml} / \mathrm{kg}$ ) and norepinephrine infusion (up to $10 \mu \mathrm{g} / \mathrm{kg}$ / minute).

Results: Hemodynamic parameters were continuously recorded. Gas exchange, acid-base status, organ function, and cytokines were measured at regular intervals until 24 hours after the onset of peritonitis when animals were sacrificed under anesthesia. Peritonitis induced profound hypotension, myocardial dysfunction, lactic acidosis, coagulation abnormalities, and multiple organ failure. These disorders were largely attenuated by LR12. In particular, cardiovascular failure was prevented as attested by better mean arterial pressure, cardiac index, cardiac power index, and $\mathrm{SvO}_{2}$, despite lower norepinephrine requirements (Figure 1). Finally, 24 -hour mortality rates were respectively $60 \%$ and $0 \%$ for control and LR12 groups.

Conclusion: LR12, a TLT-1 derived peptide, exhibits salutary properties during septic shock in adult mini-pigs.

\section{Reference}

1. Derive M, Bouazza Y, Sennoun N, Marchionni S, Quigley L, Washington V, Massin F, Max JP, Ford J, Alauzet C, Levy B, McVicar DW, Gibot S: Soluble TREM-like transcript-1 regulates leukocyte activation and controls microbial sepsis. J Immunol 2012, 188:5585-5592. 

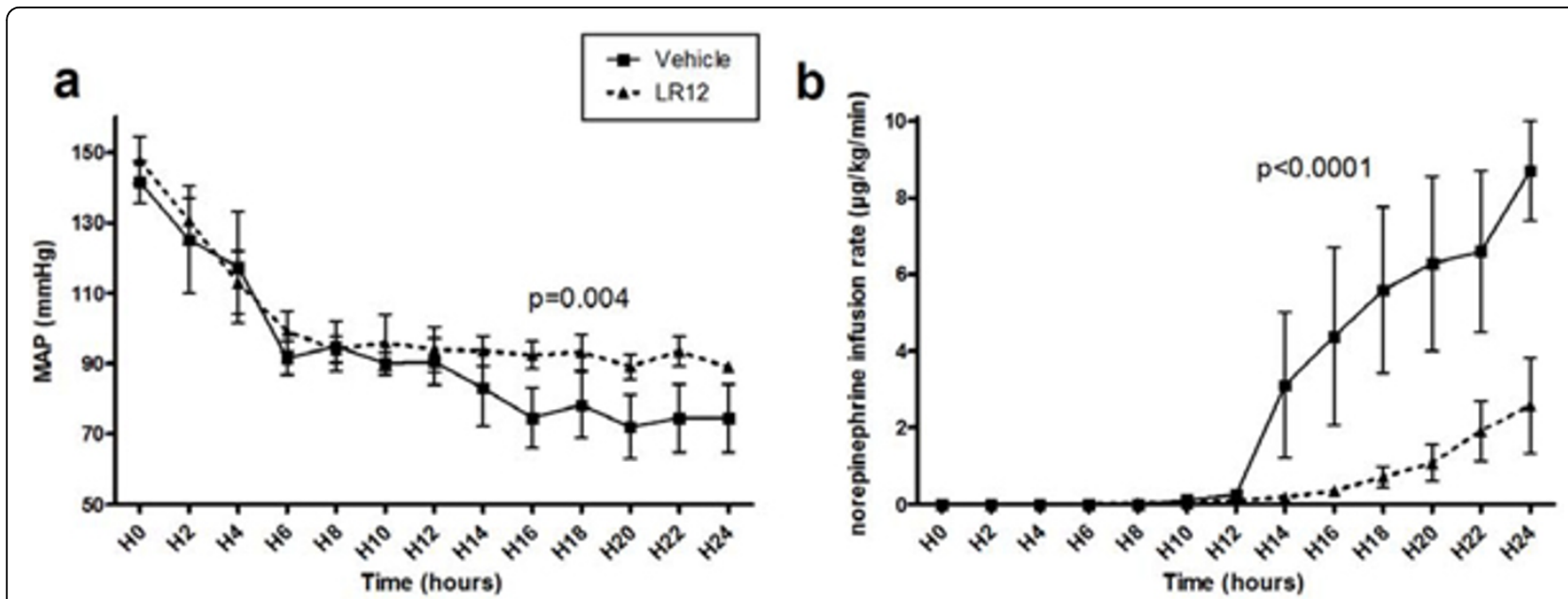

Figure 1(abstract P48) LR12 protects from sepsis-induced hypotension. Evolution of mean arterial pressure (a) and norepinephrine requirements (b) during the 24-hour study period. MAP was constantly higher and norepinephrine dose lower in LR12-treated animals than controls.

P49

Role of TREM-1 in endothelial dysfunction during experimental sepsis

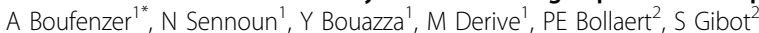

'Groupe CHOC - Inserm U961, Nancy University, Nancy, France; ${ }^{2} \mathrm{CHU}$ Nancy, France

Critical Care 2012, 16(Suppl 3):P49

Background: Triggering receptor expressed on myeloid cells-1 (TREM-1) is a receptor of the immunoglobulin superfamily expressed on the surface of neutrophils and monocytes/macrophages. It plays an important role during sepsis by amplifying the inflammatory response. Modulation of TREM-1 through the administration of a short synthetic peptide (LR12) increases survival during experimental sepsis. This study aimed to explore the mechanisms by which LR12 prevented sepsis-induced cardiovascular dysfunction.

Methods: We studied the effect of TREM-1 modulation by a synthetic peptide LR12 $(3 \mathrm{mg} / \mathrm{kg})$ on MAP and blood lactate during experimental sepsis (CLP). Aortic and mesenteric arterial vessels of these animals were collected to study the vasoreactivity to phenylephrine (Phe) and acetylcholine (Ach) ex vivo (Myograph). Alternatively, vasoreactivity was studied in the vessels of healthy animals (with and without endothelial lawyer) stimulated with LPS or with a specific agonist of TREM-1 ( $\alpha$ TREM$1,10 \mu \mathrm{g} / \mathrm{ml})$, with or without LR12 $(20 \mathrm{mg} / \mathrm{ml})$. The effect of LR12 on arterial vessels was also studied through western blotting (eNOS, iNOS, Akt, COX-1, COX-2) and qRT-PCR. Mouse lung microvascular endothelial cells (LUMECs; $\mathrm{CD} 146^{+}$) were analyzed by flow cytometry, qRT-PCR, and ELISA to decipher the effect of LR12 on LPS-induced endothelial activation.

Results: CLP induced MAP decrease and lactate elevation were prevented by the administration of LR12. Arterial vessels from septic animals treated with LR12 showed better response to Phe and Ach compared with controls. The reactivity of aortic and mesenteric vessels (contraction and relaxation) stimulated in vitro with LPS or by $\alpha$ TREM-1 was altered: this phenomenon was reversed by LR12. LR12 restored the phosphorylation of Akt and eNOS while it reduced the activation of inducible pathways (iNOS, COX-2). FACS analysis showed that TREM-1 is constitutively expressed by LUMECs (CD146 ${ }^{+} / \mathrm{NEGFR2}^{+}$). In vivo, the expression of Trem-1 was increased in septic animals and was inducible in vitro upon stimulation with LPS. Trem-1, Tnf- $\alpha$ and II- 6 expression was upregulated by LPS; once again LR12 attenuated this upregulation. Finally, the production of several cytokines by LPS-stimulated LUMECs was decreased by LR12.

Conclusion: Here we show that TREM-1 is expressed on endothelial cells from the aorta, mesenteric artery, and microvascular endothelial cells, and that TREM-1 might be directly involved in endothelial dysfunction during experimental sepsis.
P50

LPS-induced Pellino3 degradation is mediated by p62-dependent autophagy

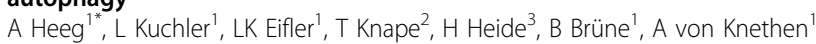
'Institute of Biochemistry I - Pathobiochemistry, Goethe-University Frankfurt,

Germany; ${ }^{2}$ Fraunhofer IME Project Group - Translational Medicine and Pharmacology, Frankfurt, Germany; ${ }^{3}$ Molecular Bioenergetics - Centre of Biological Chemistry, Goethe-University Frankfurt, Germany Critical Care 2012, 16(Suppl 3):P50

Background: In macrophages Toll-like receptor 4 (TLR4) is activated in response to lipopolysaccharide (LPS) and induces proinflammatory cytokine expression [1]. Therefore, mechanisms terminating proinflammatory gene expression are important. Autophagy plays a central role in controlling innate immune responses by lysosomal degradation of signaling proteins, thus contributing to the resolution of inflammation [2]. Autophagic proteins like p62 directly interact with molecules involved in the TLR4-signaling pathway, but a correlation with the IRAK E3 ligase and scaffold protein Pellino3 remains obscure $[3,4]$. Hence, we are interested in elucidating the function of Pellino3 to prove our hypothesis that it is a key regulator in the TLR4-signaling cascade [5].

Methods: We used the cecal ligation and puncture (CLP) mouse model causing polymicrobial sepsis to analyze Pellino3 protein and mRNA expression. Furthermore, we induced endotoxemia in RAW264.7 mouse macrophages by LPS treatment to verify in vivo experiments. Lentiviral Pellino3 knockdown in RAW264.7 macrophages was used for cytokine measurements at mRNA level. To analyze potential Pellino3 binding partners in TLR4-signaling by mass spectrometry (MS), we overexpressed FLAG-tagged Pellino3 in RAW264.7 macrophages, treated cells for 3, 6 and 24 hours with LPS and immunoprecipitated Pellino3 via its FLAG-tag. To consider Pellino3 degradation as a result of p62-mediated autophagy, we transiently knocked down p62 by siRNA in RAW264.7 macrophages and also pharmacologically blocked LPS-induced autophagy by Bafilomycin A1.

Results: We demonstrated Pellino3 protein degradation in primary CD11 ${ }^{+}$splenocytes after 24 hours following CLP operation and confirmed this in RAW264.7 macrophages after 24-hour LPS stimulation. Knockdown of Pellino3 attenuates proinflammatory cytokines, for example IL-6 mRNA, after 6 hours of LPS. Furthermore, we found by MS and verifying immunoprecipitation experiments that p62 is a Pellino3 binding partner, thus targeting Pellino3 for degradation. In line, both p62 knockdown and Bafilomycin A1 treatment prevent Pellino3 degradation, supporting an autophagic mechanism.

Conclusion: Our observations highlight a regulatory role of Pellino3 on TLR4 signaling. Thus, antagonism of Pellino3 in the hyperinflammatory 
phase of sepsis may counteract the cytokine storm. Furthermore, stabilization of Pellino3 by inhibition of autophagy in the hypoinflammatory phase of sepsis may improve immunity. In consideration of these two conflictive sepsis phases, modulation of Pellino3 may provide a new strategy for the development of a therapy approach in sepsis.

Acknowledgements: This research was supported by a grant from the Deutsche Forschungsgemeinschaft (KN493/9-1 and SFB TP3).

\section{References}

1. Zhu J, Mohan C: Toll-like receptor signaling pathways - therapeutic opportunities. Mediators Inflamm 2010, 2010:781235.

2. $\mathrm{XU}$ Y, Liu XD, Gong X, Eissa NT: Signaling pathway of autophagy associated with innate immunity. Autophagy 2008, 4:110-112.

3. Levine B, Mizushima N, Virgin HW: Autophagy in immunity and inflammation. Nature 2011, 469:323-335.

4. Schauvliege R, Janssens $S$, Beyaert R: Pellino proteins are more than scaffold proteins in TLR/LL-1R signalling: a role as novel RING E3-ubiquitin-ligases. FEBS Lett 2006, 580:4697-4702.

5. Hennessy EJ, Parker AE, O'Neill LA: Targeting Toll-like receptors: emerging therapeutics? Nat Rev Drug Discov 2010, 9:293-307.

\section{P51}

Attenuated NOX2 expression impairs ROS production during the hypoinflammatory phase of sepsis

L Kuchler $^{1 *}, V_{\text {M Morbitzer }}{ }^{1}$, A Heeg ${ }^{1}$, LK Eifler ${ }^{1}$, T Knape $^{2}$, B Brüne $^{1}$,

A von Knethen ${ }^{1}$

${ }^{1}$ Institute of Biochemistry I - Pathobiochemistry, Goethe-University Frankfurt, Germany; ${ }^{2}$ Fraunhofer IME Project Group - Translational Medicine and Pharmacology, Frankfurt, Germany

Critical Care 2012, 16(Suppl 3):P51

Background: The multicomponent phagocytic NADPH oxidase produces reactive oxygen species (ROS) after activation by microorganisms or inflammatory mediators [1]. In the hypoinflammatory phase of sepsis, macrophages are alternatively activated by contact with apoptotic cells or their secretion products. This inhibits NADPH oxidase and leads to attenuated ROS production [2] and furthermore contributes among others to a hyporeactive host defense. Due to this immune paralysis, sepsis patients suffer from recurrent and secondary infections [3]. We focused on the catalytic subunit of NADPH oxidase, the transmembrane protein NOX2 [4]. We assume that after induction of sepsis the expression of NOX2 is reduced and hence ROS production is decreased.

Methods: We induced polymicrobial sepsis in mice by cecal ligation and puncture. The ability of peritoneal macrophages (PMs) to produce ROS was determined by FACS via hydroethidine assay. NOX2 expression of PMs was determined by western blot and qPCR. To elucidate the mechanism causing mRNA destabilization, we performed in vitro experiments using J774 macrophages. To obtain an alternatively activated phenotype, macrophages were stimulated with conditioned medium from apoptotic T cells (CM). By luciferase assays we figured out a $3^{\prime} U T R$-dependent regulation of NOX2 mRNA stability. Assuming that a protein is involved in the mRNA degradation, we performed a RNA pulldown with biotinylated NOX2-3'UTR constructs followed by mass spectrometry. We verified the role of SYNCRIP by siRNA approach. Additionally, we overexpressed NOX2 in $J 774$ cells and analyzed the ROS production (w/wo CM treatment) by FACS.

Results: We found an impaired expression of NOX2 at RNA and protein level along with decreased ROS production after induction of sepsis in mice as well as stimulating $\mathrm{J774}$ macrophages with CM of apoptotic T cells. This is due to a time-dependent NOX2 mRNA degradation depending on SYNCRIP, a RNA-binding protein, which stabilizes NOX2 mRNA through binding to its $3^{\prime} U T R$ under normal conditions. In line, knockdown of SYNCRIP also decreases NOX2 mRNA expression. We assume that a CM-dependent modification or degradation of SYNCRIP prevents its stabilizing function. As the overexpression of NOX2 restores ROS production of CM-treated J774 cells, we assume that NOX2 expression is crucial for maintaining NADPH activity during the hypoinflammatory phase of sepsis.

Conclusion: Our data imply a regulatory impact of SYNCRIP on NOX2 stability during the late phase of sepsis. Therefore, further understanding of the regulation of NADPH oxidase could lead to the design of a therapy to reconstitute NADPH oxidase function, finally improving immune function in sepsis patients.
Acknowledgements: This work was supported by a grant from the Deutsche Forschungsgemeinschaft (KN493/9-1 and SFB TP3).

References

1. Bedard K, Krause KH: The NOX family of ROS-generating NADPH oxidases: physiology and pathophysiology. Physiol Rev 2007, 87:245-313.

2. Serikan BF, Gambelli F, Potapovich Al, Babu H, Di Giuseppe M, Ortiz LA, Fabisiak JP, Kagan VE: Apototic cells quench reactive oxygen and nitrogen species and modulate TNF-alpha/TGF-beta1 balance in activated macrophages; involvement of phosphatidylserine-dependent and independent pathways. Cell Death Differ 2005, 12:1141-1144.

3. Monneret G: How to identify systemic sepsis-induced immunoparalysis. Adv Sepsis 2005, 4:42-49.

4. Babior BM, Lambeth JD, Nauseef W: The neutrophil NADPH oxidase. Arch Biochem Biophys 2002, 397:342-344.

P52

Kinetic characterization of selective peroxisome-proliferator-activated receptor gamma modulators in vitro

T Knape ${ }^{1 *}$, LK Eifler ${ }^{2}$, A Heeg $^{2}$, L Kuchler $^{2}$, B Brüne ${ }^{2}$, MJ Parnham ${ }^{1}$

A von Knethen ${ }^{2}$

${ }^{1}$ Fraunhofer IME Project Group - Translational Medicine and Pharmacology,

Frankfurt, Germany; ${ }^{2}$ Institute of Biochemistry I - Pathobiochemistry,

Goethe-University Frankfurt, Germany

Critical Care 2012, 16(Suppl 3):P52

Background: The ligand-activated transcription factor, peroxisomeproliferator-activated receptor gamma (PPAR $)$, has been shown to play an essential role in immunosuppression during sepsis. PPARy is upregulated in T cells of septic patients, sensitizing these cells to PPAR $\gamma$-dependent apoptosis and thus contributing to T-cell depletion $[1,2]$. In the polymicrobial cecum ligation and puncture (CLP) sepsis model in mice, both T-cell-specific gene knockout (Lck-Cre PPAR $\gamma^{\mathrm{fl} / \mathrm{fl}}$ ) and systemic pharmacological PPAR $\gamma$ antagonism by GW9662 improved survival [3]. Because GW9662 was only effective when applied 3 hours after CLP, we were interested to extend this time frame. For this reason we characterized the kinetics of SPPAR $\gamma$ Ms when administered before or in combination with the agonist thiazolidinedione, rosiglitazone.

Methods: A PPAR $\gamma$-dependent transactivation assay was used in HEK293T cells. It is based on the vector pFA-PPAR $\gamma$-LBD-GAL4-DBD encoding the hybrid protein PPAR $\gamma$-LBD-GAL4-DBD and the reporter vector pFR-Luc, carrying a GAL4-responsive element in front of the Firefly luciferase gene. These two vectors were co-transfected, in combination with a control vector encoding Renilla luciferase (pRL-CMV) to normalize Firefly luciferase activity for transfection efficiency. Following transfection, cells were incubated with the SPPAR $\gamma$ Ms F-MOC and MCC-555 and the PPAR $\gamma$ antagonist GW9662 for different times (2 to 48 hours) and at increasing doses $(0.01$ to $10 \mu \mathrm{M})$, with or without rosiglitazone $(0.01$ to $10 \mu \mathrm{M})$. Transactivation was analyzed using a 96-well plate format.

Results: Rosiglitazone transactivated PPAR in a time-dependent and dosedependent manner, the response gradually increasing to a maximum at 48 hours with $10 \mu \mathrm{M}$. Low concentrations $(0.01$ to $0.1 \mu \mathrm{M})$ of SPPAR $\gamma \mathrm{Ms}$ F-MOC and MCC-555 and the PPAR $\gamma$ antagonist GW9662 all exerted doseindependent antagonistic effects at an early incubation time point (2 hours). From 10 hours onwards, MCC-555 and GW9662, given alone, both exerted PPAR $\gamma$ agonistic effects, MCC-555 in parallel to responses to rosiglitazone, but GW9662 with characteristics of partial antagonism. F-MOC showed no dose-dependent effect at any concentration at later time points. Only GW9662 (1 to $10 \mu \mathrm{M}$ ) was able to inhibit rosiglitazone (0.1 to $1 \mu \mathrm{M})$-induced PPAR $\gamma$ transactivation after 10 hours.

Conclusion: Our kinetic analysis reveals clear differences in the modulatory characteristics of PPAR inhibitors, with previously unreported early inhibitory effects and late agonistic or partial agonistic activity. New SPPAR $\gamma$ Ms with extended inhibitory activity may prove useful in the therapy of sepsis.

References

1. Soller M, Tautenhahn A, Brüne B, Zacharowski K, John S, Link H, von Knethen A: Peroxisome-proliferator-activated receptor gamma contributes to T lymphocyte apoptosis during sepsis. J Leukoc Biol 2006, 79:235-243.

2. Soller M, Dröse $S$, Brandt U, Brüne B, von Knethen $A$ : Mechanism of thiazolidinedione-dependent cell death in Jurkat T cells. Mol Pharmacol 2007, 71:1535-1544. 
3. Schmidt MV, Paulus P, Kuhn AM, Weigert A, Morbitzer V, Zacharowski K, Kempf VA, Brüne B, von Knethen A: Peroxisome-proliferator-activated receptory-induced $\mathrm{T}$ cell apoptosis reduces survival during polymicrobial sepsis. Am J Respir Crit Care Med 2011, 84:64-74.

P53

IL- 6 and IFN $\gamma$ play a role in fatal cases of $5 \mathrm{~N} 1$ influenza in children A Alam*, H Jusuf, CB Kartasasmita, D Setiabudi, S Sudarwati, DA Wulandari, AU Suardi, DH Somasetia

Medical Faculty Universitas Padjadjaran, Bandung, Indonesia

Critical Care 2012, 16(Suppl 3):P53

Background: Fatal human critical cases associated with influenza A subtype H5N1 have been documented in Bandung, Indonesia. Of four children, three died. We determined the level of cytokines and chemokines in those patients.

Methods: The Luminex method was used to look for the profile of cytokine and chemokine gene expression induced by $\mathrm{H} 5 \mathrm{~N} 1$ influenza virus from patient's serum.

Results: We found that $\mathrm{H} 5 \mathrm{~N} 1$ influenza virus in the dead children was a more potent inducer of IL-6, the level being higher $(17.00,74.31,85.75)$ than in the one child who survived (4.78). The IFN $\gamma$ level of the fatal causes was also higher $(21.43,31.75,384.38)$ than in the one child who recovered (5.51). This suggested that a cytokine storm may play a role in the pathogenesis of fatal $\mathrm{H} 5 \mathrm{~N} 1$ cases.

Conclusion: The H5N1 influenza A virus is a potent inducer of proinflammatory cytokines and chemokines. This hyperinduction of cytokines may be relevant to mortality of children with H5N1 infection.

\section{P54}

Abstract withdrawn

Critical Care 2012, 16(Suppl 3):P54

P55

Involvement of thrombopoietin in the development of organ injury

in a mouse model of cecal ligation and puncture-induced sepsis

E Greco*, A Cuccurullo, B Laface, D Gallo, O Bosco, P De Giuli, E Lupia,

$G$ Montrucchio

University of Turin, Italy

Critical Care 2012, 16(Suppl 3):P55

Background: Sepsis-induced organ damage is a leading cause of death in critically ill patients. Thrombopoietin (TPO) is a humoral growth factor mainly involved in regulation of platelet number and function. High circulating levels of TPO are detectable in septic adults and children and are related to sepsis severity. We have previously shown a correlation between TPO levels and platelet activation in septic burned patients, where circulating activated platelets may cause microthrombotic events that lead to organ damage. Our aim was to evaluate the contribution of TPO in organ injury in a murine model of polymicrobial sepsis. To this end, we synthesized and used a chimeric fusion protein, named mouse TPO Receptor-Maltose Binding Protein (mTPOR-MBP), able to inhibit TPO biological activity.

Methods: Male C57BL/6 mice were randomized to cecal ligation and puncture-induced sepsis (CLP) or to laparotomy (sham) surgery. CLP mice received $40 \mu \mathrm{g} \mathrm{mTPO-MBP}$ or sterile saline 10 minutes after surgery. Immediately after and 6 hours after surgery all animals received $0.08 \mathrm{mg} / \mathrm{kg}$ buprenorfin in $1.5 \mathrm{ml}$ sterile saline subcutaneously. After 18 hours blood was collected from the cava vein and used for cell count, flow cytometric analysis of leukocyte-platelet interaction and to obtain plasma. Plasma TPO levels were determined by ELISA. The lung and liver were excised and fixed in $4 \%$ paraformaldehyde solution. An expert pathologist blinded to experimental groups quantified organ damage.

Results: Plasma TPO levels were significantly higher in septic mice (nine mice in each group). Leukocyte and platelet counts did not significantly differ in the CLP group treated with mTPOR-MBP compared with the CLP control group. In contrast, the percentage of monocyte-platelet aggregates, a marker of platelet activation, was significantly reduced after treatment with mTPOR-MBP. Moreover, TPO blockade by mTPOR-MBP administration induced a significant reduction of histological alterations in the lung, as evaluated by neutrophil infiltration and thickening of the alveolar-capillary membrane, and liver tissue samples, as evaluated by the number of microabscesses.

Conclusion: Increased circulating levels of TPO during experimental sepsis may have a role in the development of organ damage. Inhibition of TPO biological activity may represent a novel promising therapeutic approach to prevent organ failure in severe sepsis.

P56

Cholecystokinin protects rats against Staphylococcus aureus-induced sepsis

RS Saia*, FMC Zuelli, EC Cárnio

University of Sao Paulo, Ribeirão Preto, São Paulo, Brazil

Critical Care 2012, 16(Suppl 3):P56

Background: Cholecystokinin (CCK) was firstly described as a gastrointestinal hormone, but immune cells express their receptors, suggesting a possible involvement of this peptide in pathophysiological processes. Our aims were to evaluate the role of CCK on resistance against Gram-positive Staphylococcus aureus-induced sepsis, as well as cell influx to infectious focus. Furthermore, since nitric oxide, TNF $\alpha$ and IL-10 play a key role in the innate immune system controlling bacterial infection, we also evaluated the synthesis of these inflammatory mediators during this sepsis model.

Methods: Male Wistar rats (180 to $200 \mathrm{~g}$ ) received an intraperitoneal injection of proglumide $(P)$ (nonselective CCK receptor antagonist; 30 or $50 \mathrm{mg} / \mathrm{kg}) 30$ minutes before bacterial $\mathrm{S}$. aureus inoculum $\left(0.5\right.$ to $1 \times 10^{10}$ CFU/animal). At 4 and 24 hours after sepsis induction, blood and peritoneal lavage fluid (PLF) were collected for microbiological analysis, cytokines and nitrate quantifications and also differential cell counting. Nitrate was detected by chemiluminescence, while TNF $\alpha$ and IL-10 were determined by ELISA sandwich kits.

Results: The pretreatment with $P$ at higher dose $(50 \mathrm{mg} / \mathrm{kg})$ increased bacteremia in comparison with the saline-injected group $(2,052 \pm 810.7$ vs. $154.3 \pm 47.0 \mathrm{CFU} / \mathrm{ml}, P<0.01)$ at 4 hours after sepsis induction. At the same time point, the bacterial counting in PLF increased in a dosedependent manner in the $P$-treated rats $(P<0.05)$. On the other hand, only the higher $\mathrm{P}$ dose elevated significantly the CFU/ml in the PLF at 24 hours $\left(97.75 \pm 12.77 \times 10^{4}\right.$ vs. $\left.35 \pm 10.05 \times 10^{4} \mathrm{CFU} / \mathrm{ml}, P<0.05\right)$. The plasma TNF $\alpha$ and nitrate concentrations were not changed by treatment or time after sepsis induction. However, the administration of CCK receptors antagonist reduced the TNF $\alpha$ production in comparison with the control group in PLF, at both time points. The plasma IL-10 concentration increased at 4 hours in P-treated rats, while at 24 hours it was reduced $(85.83 \pm 48.0$ vs. $1,698 \pm 265.6 \mathrm{pg} / \mathrm{ml}, P<0.001)$. In PLF, the rats pretreated with $P$ reduced the $\mathrm{IL}-10$ measurements $(P<0.05)$ when compared with the control group. In agreement, the macrophage influx to peritoneal infectious focus was compromised by treatment with a high $P$ dose at 24 hours after $S$. aureus-induced sepsis $(3,947.73 \pm 269.99$ vs. $5,629.61 \pm 786.90 \mathrm{cells} / \mu \mathrm{l} ; P<0.05)$. Moreover, the neutrophil count did not change among the experimental groups $(6,860.59 \pm 211.90$ vs. $6,273.54 \pm 798.91$ cells/ $\mu$ l).

Conclusion: These data suggest a protective role for CCK peptide during S. aureus-induced sepsis, modulating the systemic and local inflammatory response, as well as increasing the macrophage influx to the infectious focus.

P57

Polymyxin B-direct hemoperfusion therapy contributes to oxygen delivery in septic patients

A Yaguchi*, M Yokota, T Goto, M Takeda, R Moroi, T Harada, M Namiki

Tokyo Women's Medical University, Tokyo, Japan

Critical Care 2012, 16(Suppl 3):P57

Background: Since 1994, Polymyxin B-direct hemoperfusion (PMX-DHP) (Toraymyxin ${ }^{\oplus}$; Toray Medical Co., Tokyo, Japan) has been approved as 
a therapy for patients with severe sepsis or septic shock due to Gramnegative infection in Japan. However, its efficacy and indication are still controversial issues. Recently, randomized controlled studies are ongoing in other countries. Our hypothesis is that PMX-DHP therapy may improve the hemodynamic status in septic patients.

Methods: From July 1994 to June 2010, all adult patients treated with PMX-DHP and receiving a pulmonary arterial catheter (PAC) in our ICU were included in this retrospective observational study. Patients' clinical, microbiological and PAC data were collected from medical archives. PAC variables were compared between immediately before and immediately after PMX-DHP therapy. Values were expressed as mean \pm SD. Data were analyzed by Wilcoxon signed-ranks test, chi-square test and Fisher's exact probability test. $P<0.05$ was considered statistically significant.

Results: Sixty-three patients (36 men, 27 women; age range 24 to 89 years (mean $63.4 \pm 14.8$ )) were studied. The mortality rate was $30.2 \%$ at 28 days after PMX-DHP. The APACHE II score and SOFA score on the day of PMX-DHP therapy were $20.2 \pm 14.8$ and $7.3 \pm 3.8$, respectively. Mean arterial pressure (MAP; $\mathrm{mmHg}$ ), cardiac index $\left(\mathrm{Cl} ; \mathrm{l} / \mathrm{minute} / \mathrm{m}^{2}\right)$, and oxygen delivery and consumption $\left(\mathrm{DO}_{2}\right.$ and $\mathrm{VO}_{2} ; \mathrm{ml} /$ minute) significantly improved after PMX-DHP therapy $(77.5 \pm 22.5$ vs. $84.1 \pm 23.4, P=0.0013$; $3.9 \pm 1.5$ vs. $4.3 \pm 1.2, P=0.0006 ; 951.6 \pm 403.5$ vs. $1,002.5 \pm 394.0, P=$ $0.013 ; 233.5 \pm 80.6$ vs. $248.0 \pm 84.1, P=0.004$, respectively). The systemic venous resistance index (SVRI) (dyn second $\mathrm{m}^{2} / \mathrm{cm}^{5}$ ) and mixed venous oxygen saturation $\left(\mathrm{SvO}_{2} ; \%\right)$ were not statistically different before and after PMX-DHP therapy $(1,573.6 \pm 614.6$ vs. $1,527.7 \pm 575.5, P=0.069$; $71.9 \pm 9.0$ vs. $74.1 \pm 7.5, P=0.082)$. The inotropic score and $\mathrm{P} / \mathrm{F}$ ratio improved after the therapy $(9.9 \pm 15.9$ vs. $7.5 \pm 12.5, P=0.018 ; 269.8 \pm$ 108.5 vs. $300.6 \pm 133.4, P=0.003$ ).

Conclusion: $\mathrm{MAP}, \mathrm{Cl}, \mathrm{DO}_{2}, \mathrm{VO}_{2}$, inotropic score and $\mathrm{P} / \mathrm{F}$ ratio improved after PMX-DHP therapy, while SVRI and $\mathrm{SVO}_{2}$ did not change. PMX-DHP could contribute to oxygen delivery due to improve hemodynamic status, while decreasing inotropic agents in septic patients.

P58

Sepsis in neonates: experience in a tertiary-care hospital

I Guerrero-Lozano*, A Alonso-Ojembarrena, F Galán-Sanchez, AM García-Tapia, P Marin-Casanova, P García-Martos, M Rodriguez-lglesias

Puerta del Mar University Hospital, Cadiz, Spain

Critical Care 2012, 16(Suppl 3):P58

Background: Sepsis is a common condition in newborns, which has significant morbidity and mortality worldwide. In recent years, multiple factors have led to an increase in its incidence, such as the use of invasive diagnostic procedures, broad-spectrum antimicrobial therapy and an increase of immunocompromised patients. There have also been changes in the profile of the agents causing sepsis. The aims of this study were the determination of the annual incidence of neonatal sepsis (early-onset and late-onset), analyzing their clinical course, significant microorganisms isolated and the profile of antimicrobial resistance.

Methods: One hundred and ninety-eight cases of sepsis were studied, collected over the past five years (2007 to 2011). Early-onset neonatal sepsis was defined as that which appeared before 3 days of life and lateonset neonatal sepsis if it happened later. Blood cultures were incubated in the BACTEC FX System (Becton Dickinson), and identification and antimicrobial susceptibility testing were done by the Wider system (Soria Melguizo). Yeasts were identified by ID32C (bioMerieux).

Results: Of the 198 detected sepsis cases, $173(87.4 \%)$ were late and 25 $(12.6 \%)$ were early. While the annual incidence rate of early-onset sepsis was uniform in each year, the late-onset sepsis has experienced an increase in 2011. The mortality rate was similar throughout the study (10.8\%). Gram-negative bacilli are the most frequently isolated (48\%), with predominance of enterobacteria compared with nonfermenters (84 vs. 11 cases), followed by Gram-positive cocci (36\%) and yeast (16\%). In candidemia most common are nonalbicans species of Candida (27 vs. 5 cases), with Candida parapsilosis more frequently isolated (65.6\%). Klebsiella pneumoniae (33 strains) was the more frequent microorganism, followed by Escherichia coli, Staphylococcus epidermidis, C. parapsilosis and Enterobacter cloacae. With respect to antibiotic resistance, $11.1 \%$ of Staphylococcus aureus were MRSA and 19\% (16 strains) of enterobacteria were ESBL producing. Only one strain of Pseudomonas aeruginosa was resistant to imipenem by metallobetalactamases.
Conclusion: K. pneumoniae was the organism responsible for more episodes of sepsis in neonates. As in other studies in neonatology, this study highlights the prevalence of nonalbicans species in candidemia due to the frequency of $C$. parapsilosis. Late-onset sepsis has increased by Gram-negative bacilli in the last year, probably due to the occurrence of a nosocomial outbreak of ESBL-producing E. cloacae. However, the mortality rate from sepsis has remained stable. When clinically evaluated, cases of sepsis can be detected as a predominance of enterobacteria against $S$. epidermidis, highlighting the importance of careful analysis of clinical outcomes.

\section{P59}

Is urinary kidney injury molecule-1 a good marker for acute kidney injury in septic shock?

J Göschl", L Sudhoff, M Koehler, W Rachinger, J Briegel, I Kaufmann

Ludwig-Maximilians-University of Munich, Germany

Critical Care 2012, 16(Suppl 3):P59

Background: Kidney injury molecule-1 (KIM-1) is a new biomarker promising a better detection and diagnostic quality of acute kidney injury (AKI).

Methods: This clinical prospective study - approved by the local ethics committee - was performed to assess urinary levels of KIM-1 by ELISA technique in 38 patients with septic shock (Table 1). They were prospectively enrolled within 24 hours of onset of signs of infection, if they met the criteria for septic shock as defined by the members of the ACCP/SCCM Consensus Conference Committee. KIM-1 levels were quantified on admission (baseline, day 0 ) and on days 4, 7 and 10 of the ICU stay. The patients were classified by AKIN and RIFLE criteria. Data were analyzed with regard to the prognostic value of survival, need for renal replacement therapy (RRT) and correlation with renal function parameters (plasmatic urea and creatinine, creatinine elimination and clearance, free water clearance, fractional sodium excretion) or hepatic laboratory findings (albumin, total bilirubin, ASAT, ALAT, $\gamma-$ GT). Data are given as mean \pm SEM.

Results: The urinary KIM-1 concentration on admission showed no significant difference with respect to survival of patients. However, the urinary KIM-1 concentration determined on days 4, 7 and 10 was higher in patients surviving septic shock $(P<0.001$ on days 4 and 7$)$. Elimination displayed lower levels in deceased patients $(P<0.05$ on days 0 and 4$)$, whereas urinary output was higher in survivors during the whole ICU stay $(P<0.05$ on day 7). Urinary KIM-1 concentration did not differ between AKIN and RIFLE classification groups. Urinary KIM-1 elimination per 24 hours on days 0,4 and 7 was higher in stage 1 than in stage 2 or 3 of the AKIN classification, respectively (both $P<0.05$ ). If patients were classified by RIFLE criteria, urinary KIM-1 elimination was also higher in the risk group as compared with the injury or failure group without reaching significance. However, the need for RRT was reflected by a higher urinary KIM-1 concentration after admission ( $P<0.05$ on days 4 and 10$)$, a lower KIM-1 elimination and urinary output during the whole ICU stay (KIM-1 elimination:

\section{Table 1(abstract P59) Demographic data}

\begin{tabular}{ll}
\hline Age (years) & $57.2 \pm 2.6$ \\
Gender (male/female) & $27 / 11$ \\
BMI $\left(\mathrm{kg} / \mathrm{m}^{2}\right)$ & $29.7 \pm 1.5$ \\
Length of ICU stay (days) & $19.5 \pm 2.7$ \\
Mortality (\%) & 13.2 (5 out of 38 patients) \\
SAPS score & $63.4 \pm 2.7$ \\
APACHE II score & $28.8 \pm 1.3$ \\
SOFA score & $12.7 \pm 0.5$ \\
MOD score & $10.4 \pm 0.6$ \\
RIFLE (number per group) & no AKI $=28 ;$ risk = 4; injury = 3; \\
AKIN (number per group) & no AKI $=25 ;$ stage 1 = 1; stage 2 = 1; \\
& stage $3=11$ \\
Need for RRT (yes/no) & $16 / 22$ \\
\hline
\end{tabular}



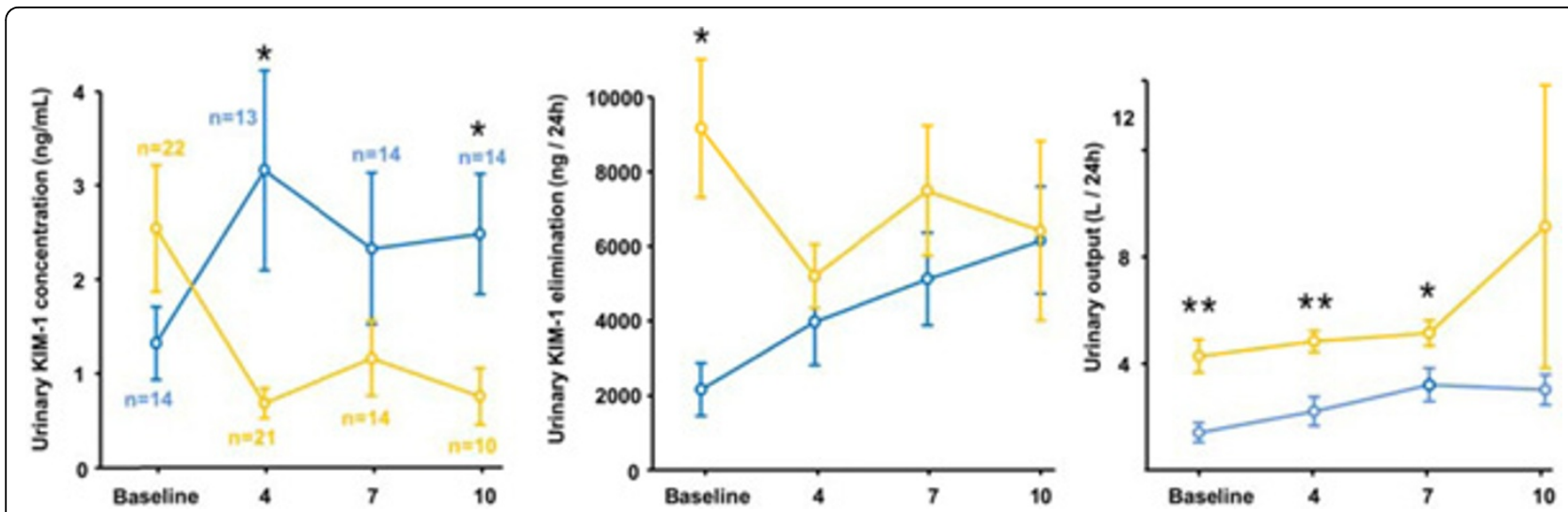

Figure 1(abstract P59) Urinary KIM-1 concentration (A) and elimination (B) in comparison with urinary output (C) per 24 hours in patients with (blue line) and without (yellow line) RRT at baseline and on days $\mathbf{4 , 7}$ and 10. ${ }^{*} P<0.05$, ${ }^{* *} P<0.001$.

$P<0.05$ on day 0 ; urinary output: $P<0.001$ on days 0 and $4, P<0.05$ on day 7 ; Figure $1 \mathrm{~A}, \mathrm{~B}, \mathrm{C})$. Neither urinary KIM-1 concentration or elimination correlated with any renal and hepatic function parameter.

Conclusion: Urinary KIM-1 can be used as a prognostic factor for survival and need for RRT in septic shock patients.

P60

Necrotizing fasciitis: modern clinical view

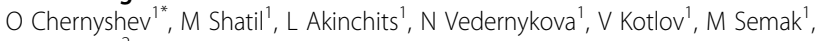
N Bubnova ${ }^{2}$

${ }^{1}$ City Hospital of St. George, Saint-Petersburg, Russia; ${ }^{2}$ Saint-Petersburg State Medical University of I.P. Pavlov, Saint-Petersburg, Russia

Critical Care 2012, 16(Suppl 3):P60

Background: Necrotizing fasciitis (NF) is one of the most severe diseases among surgical infections. There is always severe sepsis, multiple organ dysfunction, septic shock with NF and high mortality rates from $50 \%$ to 80 to $100 \%$. Progressive severe sepsis with a lack of local inflammatory symptoms is being characterized as a modern clinical view of NF.

Methods: A 5-year retrospective analysis of 12,907 admitted patients to the Department of Surgical Infections and Sepsis from 2007 until 2011 years was performed. All patients were admitted by the emergency service. The duration of admitting to the hospital from disease onset was 1 to 7 days, with a mean of 2 to 3 days.

Results: There were 86 patients with NF in 2007 to 2011. All patients had severe sepsis or septic shock with multiple organ dysfunction proved by laboratory tests and were treated in the ICU. The most frequent accompanying immunodeficient disorders are diabetes, oncology, alcohol and drug addiction, and extensive contusion of soft tissues. There were six patients in 2007 with NF (2 discharged/4 died), 13 patients in 2008 (7/6), 18 patients in 2009 (6/12), 16 patients in 2010 (12/4), and 33 patients in $2011(15 / 18)$ correspondingly. Obtained results show a stable increase of patients with NF. The amount of patients with NF by 2011 was extended 5.5 times compared with 2006. The mortality rates fluctuated between 55 and $66 \%$, less mortality rate was seen in 2010 (25\%). On average the mortality rate was $51.5 \%$; this corresponds to the modern literature reviews. The most common organisms isolated were: Enterococcus faecalis (18.5\%), Staphylococcus aureus (15.5\%), Streptococcus (14\%) and anaerobic cultures (6.6\%). All admitted patients had been treated immediately with high doses of intravenous antibiotics and extensive surgical treatment of the infection lesions; following intensive treatment in the ICU was made. Serious attention to immunotherapy was made. Severe reduction of the lymphocytes and hemoglobin seemed clinically significant in prediction of inflammation severity.

Conclusion: Accompanying immunodeficient disorders influence rising NF. Early diagnostics and active treatment make better treatment results and decrease mortality rates. Progressive decrease of lymphocytes and hemoglobin is extremely adverse to predict. Using immunotherapy makes clinical results much better. Regular microbiological monitoring of wound infection should make adequate antibiotic therapy.

P61

Erysipelas: complement system and SIRS

O Chernyshev ${ }^{1 *}$, A Petrov $^{2}$, A Shatil ${ }^{1}, N$ Serebryanaya ${ }^{2}$, N Bubnova $^{3}$

${ }^{1}$ City Hospital of St. George, Saint-Petersburg, Russia; ${ }^{2}$ State Research Institute of Highly Pure Biopreparations, Saint-Petersburg, Russia; ${ }^{3}$ Saint-Petersburg

State Medical University of I.P. Pavlov, Saint-Petersburg, Russia

Critical Care 2012, 16(Suppl 3):P61

Background: Erysipelas in some patients can be complicated with SIRS and sepsis, even with septic shock that may be explained by the hyperactivity of the complement system. The complement system in erysipelas has not been explored thoroughly. Previously a reduction of the general hemolytic activity of different complement components was reported. The study of the complement system is absolutely required to determine the functional state of the immune system and inflammation process in erysipelas.

Methods: Plasma samples from 38 patients with different clinical erysipelas forms (two primary erysipelas, 12 recurrent erysipelas) were obtained in 2010. The erythematous form was diagnosed in 10 patients with primary infection and in four patients with recurrent infection, the erythematous-hemorrhagic form in eight and seven patients, and the bullous-hemorrhagic form in seven and two patients accordingly. Blood samples were taken on day 1 (admission to the hospital) and on days 7 to 10 (on discharge from the hospital). The evaluation of the concentration of the complement components was performed by commercial solid-phase ELISA. The components studied were: C1-inhibitor $(\mathrm{C} 1-\mathrm{INH})(\mu \mathrm{g} / \mathrm{ml})$, C4 $(\mathrm{mg} / \mathrm{ml}), \mathrm{C} 3(\mathrm{mg} / \mathrm{ml}), \mathrm{C} 5(\mu \mathrm{g} / \mathrm{ml})$, anafilatoxins C3a $(\mathrm{ng} / \mathrm{ml})$ and C5a $(\mathrm{ng} /$ $\mathrm{ml})$, and a hydrolysed form of the $\mathrm{C} 3$ component $\mathrm{C}_{3}\left[\mathrm{H}_{2} \mathrm{O}\right](\mathrm{mg} / \mathrm{ml}$ and $\%$ of total (3) that is triggered by the alternative pathway.

Results: There were significantly increased concentrations of anafilatoxins $\mathrm{C} 3 \mathrm{a}$ and $\mathrm{C} 5 \mathrm{a}, \mathrm{C} 1-\mathrm{NNH}$ and $\mathrm{C}_{3}\left[\mathrm{H}_{2} \mathrm{O}\right]$ in erysipelas patients compared with healthy individuals. Correlations were found between serum concentrations of $\mathrm{C} 4$ and $\mathrm{C} 3(r=0.89 ; P<0.01)$ and anafilatoxins $\mathrm{C} 3 \mathrm{a}$ and $\mathrm{C} 5 \mathrm{a}$ ( $r=$ $0.55 ; P<0.01)$. Significant differences were found in different clinical erysipelas forms: patients with primary erysipelas (erythematous form) had significantly higher concentrations of anafilatoxin $C 3$ a than patients with the more severe bullous-hemorrhagic form $(P=0.02)$. These data support the hypothesis that $\mathrm{C} 3 \mathrm{a}$ has regulatory anti-inflammatory features that make it differ from the definitely proinflammatory C5a component. The portion of $\mathrm{C}_{3}\left[\mathrm{H}_{2} \mathrm{O}\right]$ was significantly lower in the group of patients with recurrent erysipelas (erythematous form) than in the combined group of erythematous-hemorrhagic and bullous-hemorrhagic erysipelas forms ( $P=$ 0.04 ). This suggests that spontaneous complement activation via an alternative pathway is less frequent in patients with mild erysipelas forms. After the treatment period we observed a significantly decreased 
percentage of $\mathrm{C}_{3}\left[\mathrm{H}_{2} \mathrm{O}\right]$ in patients with primary (bullous-hemorrhagic form) erysipelas $(P=0.02)$ and in patients with recurrent erysipelas (erythematous-hemorrhagic and bullous-hemorrhagic forms) erysipelas $(P=0.02)$. This proves modern knowledge about decreasing complement activity during reduction of inflammation.

Conclusion: It was shown that the complement system is significantly activated in erysipelas patients due to increased concentrations of $\mathrm{C} 3 \mathrm{a}$ ( $\sim 3$ to 5 times), C5a ( 1.5 times), C1-INH ( 1.5 times) and \% C3[ $\left.\mathrm{H}_{2} \mathrm{O}\right]$ ( $\sim 100$ times) compared with healthy individuals. We suggest that investigation of the complement activity may allow one to improve the estimation of inflammation severity, and predict development of SIRS and effectiveness of therapy.

\section{P62}

Pancreatic stone protein: a new predictor of outcome in patients with peritonitis

R Gukasjan ${ }^{1 *}$, D Raptis ${ }^{2}$, HU Schulz ${ }^{1}$, W Halangk ${ }^{1}$, R Graf $^{2}$

${ }^{1}$ Otto-von-Guericke University, Magdeburg, Germany; ${ }^{2}$ University Hospital Zurich, Switzerland

Critical Care 2012, 16(Suppl 3):P62

Background: Infection and sepsis are serious postoperative complications, which have to be recognized and treated by appropriate therapeutic options in the ICU. Pancreatic stone protein (PSP/reg) is synthesized and secreted by the pancreas, the stomach and the small intestine. In this study we evaluated whether PSP/reg predicts sepsis-related postoperative complications and death in patients with peritonitis.

Methods: A prospective cohort study of postoperative patients admitted to the ICU in an adult surgical teaching hospital in Germany. Ninety-one consecutive postoperative patients with proven diagnosis of secondary peritonitis admitted to the ICU were included in the study. Blood samples were taken within 3 hours from admission to the ICU for analysis of PSP/reg, white blood cell counts (WCC), C-reactive protein (CRP), IL-6, and procalcitonin (PCT). The Mannheim Peritonitis Index and APACHE II clinical scores were also determined. Univariate and multivariate analyses were performed to determine the diagnostic accuracy and independent predictors of death in the ICU.

Results: Univariate analysis demonstrated that PSP/reg has the highest diagnostic accuracy for complications such as organ failure and is the best predictor for death in the ICU. PSP/reg had the highest overall efficacy in predicting death with an odds ratio (OR) of 4.0 versus PCT (OR 3.2), IL-6 (OR 2.8), CRP (OR 1.3), and WCC (OR 1.4). By multivariate analysis, PSP/reg was the only independent predictor of death.

Conclusion: Patients with sepsis-related complications showed elevated serum-PSP/reg levels. PSP/reg demonstrated a high diagnostic accuracy to discriminate the severity of peritonitis and to predict death in the ICU. This test could be used in clinical diagnosis and therapeutic decisionmaking in the ICU.

Acknowledgements: This study was supported by the Gebert Rüf Foundation, Switzerland.

\section{P63}

Audit on patient outcome based on APACHE II scoring in the respiratory ICU of a south Indian university teaching hospital

H Mangu*, M Anakapalli, A Samantaray

Sri Venkateswara Institute of Medical Sciences, Tirupati, India

Critical Care 2012, 16(Suppl 3):P63

Background: The Acute Physiology and Chronic Health Evaluation (APACHE II) score is widely used in the ICU and has been well validated against the other critical illness scoring systems in predicting ICU mortality. The aim of this audit was to determine survival to hospital discharge for patients admitted to our ICU during a period of 6 months.

Methods: We performed a prospective audit of 80 consecutive patients admitted to our 16-bed respiratory intensive care unit (RICU) in a university teaching hospital. Patients were excluded if the observation period was less than 24 hours and age less than 12 years. An audit form was completed by a fellow intensivist who followed up the patients until they were discharged from the RICU. Patient data were stratified according to the outcome (S, survivors; $E$, expired) and compared for different age groups (12 to 29 years, 30 to 60 years, $>60$ years), gender (males vs. females) and diagnostic disease groups. Student's $t$ test, chi-square test/ Fisher's exact test and analysis of variance were used for comparison of data as appropriate and $P<0.05$ was considered significant.

Results: The mean APACHE II score, mean age and duration of ICU stay were $12.4 \pm 10.8,42.1 \pm 20.6$ years and $12.8 \pm 12.2$ days respectively. The patients' number of days of ICU stay was independent of patients' outcome ( $\mathrm{S}$ or $\mathrm{E})(P=0.476)$. Similarly the gender distribution did not affect the mean APACHE II score on admission $(P=0.273)$ and duration of ICU stay $(P=0.166)$. The survival rate among the eight different diagnostic groups was similar $(P=0.064)$. A higher APACHE II score and a higher age was associated with increased mortality $(P<0.001$ and $P=0.001$ respectively).

Conclusion: Our RICU had a mortality of $32 \%$ (26/80 patients) and patients who died belonged to the higher age group and had high mean APACHE II score. Although our audit is small and may not represent the cohort adequately, our mortality rate was similar to that obtained from a large cohort [1]. The higher mortality rate was probably because our centre is a tertiary-care referral hospital and many referred cases reached us late.

Reference

1. Knaus WA, Draper EA, Wagner DP, Zimmerman JE: APACHE II: a severity of disease classification system. Crit Care Med 1985, 13:818-829.

\section{P64}

Internal jugular vein catheterization: a comparative study of apical and paracarotid approaches

H Mangu*, A Samantaray

Sri Venkateswara Institute of Medical Sciences, Tirupati, India

Critical Care 2012, 16(Suppl 3):P64

Introduction: Central venous catheterization (CVC) is a frequently performed procedure in ICUs for both monitoring and definitive central venous access. Although the apical approach is the most preferred technique in our practice, a modified landmark guided technique that uses only the carotid artery pulsation as a landmark (paracarotid approach) to locate the puncture site for internal jugular venous (IJV) catheterization attained a high success rate with few complications. The aim of the study was to compare two approaches used for IJV cannulation: the apical approach and the paracarotid approach. The primary endpoint was the rate of success. The secondary endpoints were the related adverse events and the difficulty factors (number of attempts).

Methods: A prospective, randomized clinical trial in a tertiary-care university teaching hospital. After obtaining approval from the Hospital Ethics Committee, 50 adult patients admitted to our ICU with an absolute indication for CVC were randomized to undergo one of the two techniques. We compared the demographics, success rates, difficulty factors and adverse events.

Results: The first-attempt success rate $(80 \%$ vs. $60 \%, P<0.05)$ and overall success rate $(92 \%$ vs. $88 \%)$ was higher in the paracarotid group, with fewer attempts ( $1.08 \pm 0.5$ vs. $1.16 \pm 0.6)$. There were no pneumothorax (0 vs. $5 \%)$, and less accidental arterial punctures (5\% vs. $18 \%$ ) in the paracarotid group, but the difference was not significant statistically.

Conclusion: This study shows that the paracarotid approach of IJV cannulation is a better technique in providing a higher first-attempt success rate and has fewer complications.

P65

Citrate anticoagulation protocol to treat septic shock patients

with liver dysfunction in CPFA extracorporeal therapy

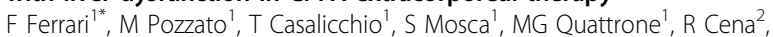

F Quarello', S Livigni ${ }^{1}$

${ }^{1}$ San Giovanni Bosco Hospital Turin, Italy; ${ }^{2}$ Bellco, CRC, Mirandola, Italy Critical Care 2012, 16(Suppl 3):P65

Background: Regional citrate anticoagulation has emerged in critically ill patients to safely treat patients with risk of bleeding. However, liver dysfunction may lead to citrate accumulation and change patients' acidbase status. In 10 years, 100 septic shock patients were treated with CPFA, an extracorporeal therapy that combines unselective plasma adsorption 
Table 1(abstract P65) Protocol scheme for citrate-calcium chloride infusion

\begin{tabular}{llllllllllll}
\hline $\mathrm{kg}$ & 50 & 55 & 60 & 65 & 70 & 75 & 80 & 85 & 90 & 95 & 100 \\
Pre ml/hour & 2,250 & 2,250 & 2,250 & 2,250 & 2,250 & 2,250 & 2,250 & 2,250 & 2,250 & 2,250 & 2,250 \\
Post $\mathrm{ml} /$ hour & 100 & 150 & 200 & 250 & 300 & 350 & 400 & 450 & 500 & 550 & 600 \\
$\mathrm{CaCl} \mathrm{10 \%} \mathrm{ml/hour}$ & 4 & 4 & 4 & 3 & 3 & 3 & 3 & 3 & 2 & 2 & 2 \\
\hline
\end{tabular}
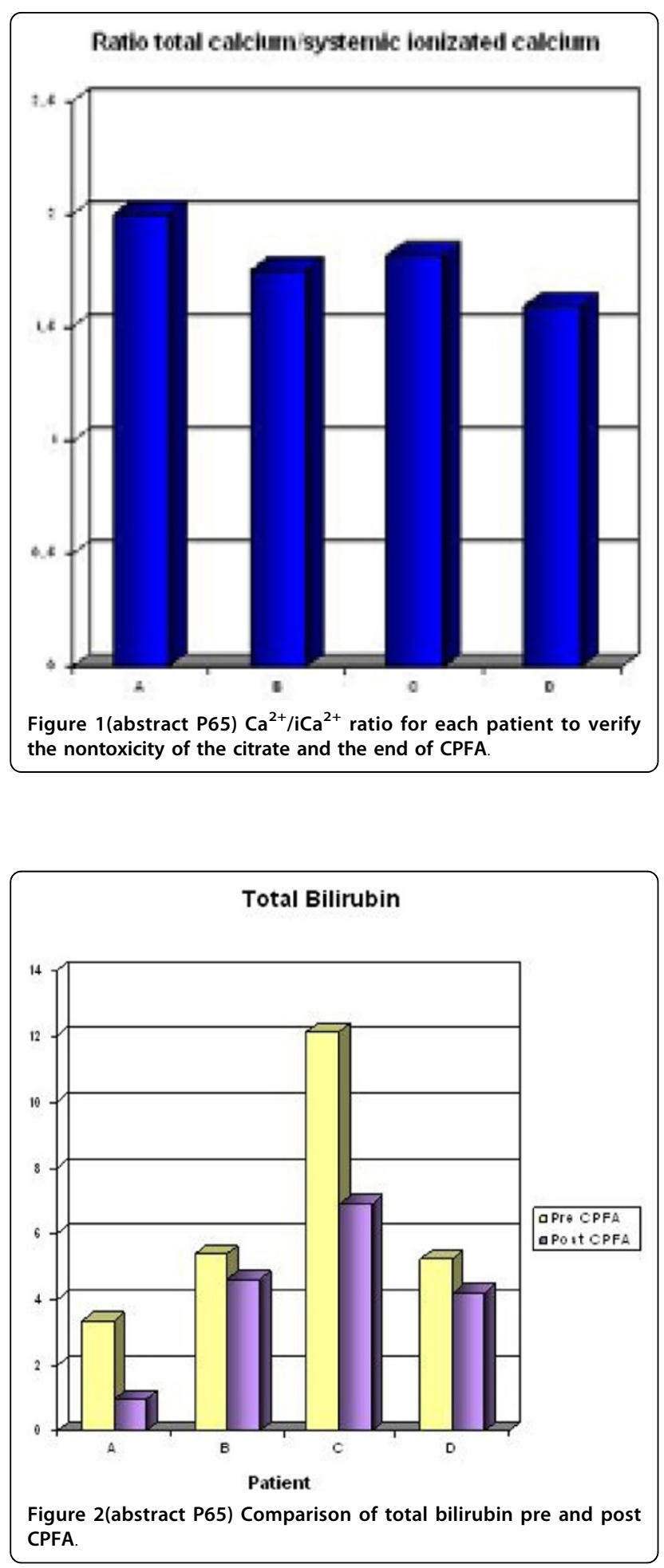

resin (MediaSorb) with continuous hemofiltration. Some patients suffered from liver dysfunction (total bilirubin $\geq 2 \mathrm{mg} / \mathrm{dl}$ ) and we treated them with our citrate protocol. The aim of this study is to evaluated the safety of citrate-like anticoagulation on patients with liver dysfunction.

Methods: Four consecutive mechanically ventilated patients (three male, one female) with septic shock and liver dysfunction were treated. Prescribed CPFA parameters were: $\mathrm{Qb} 150 \mathrm{ml} /$ minute, plasma flow rate (Qp) $30 \mathrm{ml} /$ minute, predilution solution $\left(\mathrm{Na}^{+} 136 \mathrm{mmol} / \mathrm{l}\right.$, citrate $10 \mathrm{mmol} / \mathrm{l}$, citric acid $2 \mathrm{mmol} / \mathrm{l}$ ) infused to keep inlet citratemia at $3 \mathrm{mmol} / \mathrm{l}$, postdilution solution $\left(\mathrm{Na}^{+} 139 \mathrm{mmol} / \mathrm{l}, \mathrm{K}^{+} 1.5 \mathrm{mmol} / \mathrm{l}, \mathrm{Ca}^{2+} 2 \mathrm{mmol} / \mathrm{l}, \mathrm{Mg}^{2+}\right.$ $0.75 \mathrm{mmol} / \mathrm{l}, \mathrm{HCO}_{3}^{-} 35 \mathrm{mmol} / \mathrm{l}$, glucose $\left.5.55 \mathrm{mmol} / \mathrm{l}\right)$ and postdilution $\mathrm{CaCl}_{2}$ at a rate restoring the plasma $\mathrm{Ca}^{2+}$ to $1.1 \mathrm{mmol} / \mathrm{l}$ (Table 1) and adjusted according to patients' need. We evaluated five clinical parameters pre and post CPFA: $\mathrm{pH}$, bicarbonate, lactate, $\mathrm{Ca}^{2+} / \mathrm{iCa}^{2+}$, total bilirubin.

Results: Twenty-two treatments were performed accounting for 163 hours, mean duration $10.2 \pm 2.1$ hours, mean plasma volume of $16.4 \pm 5.4 \mathrm{I}, \mathrm{Ob}$ $150 \mathrm{ml} / \mathrm{minute}, \mathrm{Qp} 28.4 \pm 1.8 \mathrm{ml} / \mathrm{minute}$, and a treated plasma dose $/ \mathrm{kg}$ body weight of $0.8 \pm 0.4 \mathrm{l} / \mathrm{kg}$. Mean $\mathrm{CaCl}_{2} 10 \%$ infusion of $4.5 \pm 1.3 \mathrm{ml} /$ hour, with citratemia, evaluated as total $\mathrm{Ca}^{2+} / \mathrm{iCa}^{2+}$ ratio (Figure 1), was always $<2.5$ (mean $1.8 \pm 0.2$ ). For pH (mean pre $7.45 \pm 0.06$ vs. mean post $7.39 \pm$ 0.07 ), bicarbonate ( $24.6 \pm 3.5$ vs. $24.8 \pm 4.7)$, and lactate $(2.9 \pm 1.5$ vs. $2.1 \pm$ 1.6) there are no statistically significant differences between pre and post treatment. Instead we observed a decrease of bilirubin (Figure 2). Mean SOFA and SAPS II pre CPFA were $14 \pm 3$ and $54 \pm 17$, respectively. During treatment, the acid-base patients' status were kept under control with no significant electrolyte correction $\left(\mathrm{Mg}^{2+}, \mathrm{K}^{+}\right.$, Thamesol 3.6\%, $\left.\mathrm{NaHCO}_{3} 8.4 \%\right)$. Conclusion: In these four patients treated with CPFA and citrate in liver dysfunction, we have observed the absence of alteration: $\mathrm{pH}$, bicarbonate, lactate and $\mathrm{Ca}^{2+} / \mathrm{iCa}^{2+}$. We also observed a decrease of bilirubin.

\section{P66}

Manipulation of nitric oxide levels with a modified hydroxyethyl starch molecule

C Lupp ${ }^{1^{*}}$, S Baasner ${ }^{1}$, D Heckmann², C Ince ${ }^{3}$, F Nocken ${ }^{1}$, M Schimmel ${ }^{2}$ M Westphal ${ }^{1}$

${ }^{1}$ Fresenius Kabi Deutschland GmbH, Bad Homburg, Germany; ${ }^{2}$ Fresenius Kabi Deutschland $\mathrm{GmbH}$, Friedberg, Germany; ${ }^{3}$ Academic Medical Center,

Amsterdam, the Netherlands

Critical Care 2012, 16(Suppl 3):P66

Background: As mortality of patients with severe sepsis and septic shock is still inappropriately high [1], innovative therapeutic approaches are urgently needed. In the presence of hypovolemia, fluid therapy is typically initiated to compensate for intravascular volume deficits [2]. However, administration of fluids may not necessarily correct a disturbed blood flow on the microcirculatory level [3]. Microcirculatory failure during sepsis is, at least in part, caused by pathological nitric oxide (NO) levels $[4,5]$. To achieve an optimal NO availability, approaches including NO donors or inhibitors may be useful. The aim of the present study was to assess the ability of our test substance S-nitrosothiol-HES (S-NO-HES) to act as NO donor and exert a pharmacological activity.

Methods: The investigated test substance S-NO-HES is a novel molecule consisting of NO coupled to a thiolated derivative of hydroxyethyl starch (HES). The ability of S-NO-HES to release NO was demonstrated. Furthermore, the effect of S-NO-HES on myocardial function was studied in isolated Langendorff-perfused hearts from guinea pigs and compared with that of the reference substance sodium nitroprusside. The thiolated HES derivative (SH-HES) served as negative control. In addition, isolated aortic rings from rats were pre-contracted by phenylephrine. After defined incubation periods with reference, test, or control items, the NO-induced relaxation was determined. S-nitrosoglutathione served as reference compound, HES and in one experiment also SH-HES as control substances. 
At the end of the 180-minute experiment, papaverine was applied in order to completely relax the aortic rings and to define the $100 \%$ relaxation level. Results: S-NO-HES significantly increased the heart rate of Langendorffperfused guinea pig hearts and additionally reduced both the QT interval and QTC-B values. In addition, S-NO-HES exerted a significant vasodilatory effect on phenylephrine pre-contracted rat aortic rings that was dose dependent. The effect was not only observed under light, which is known to trigger NO release from S-nitroso compounds, but also under exclusion of light and therefore more physiological conditions.

Conclusion: We demonstrated for the first time that the S-NO-HES molecule released NO and exhibited corresponding pharmacological properties. In future experiments, the effectiveness of S-NO-HES to substitute NO deficiency under septic conditions has to be studied. References

1. Melamed A, Sorvillo FJ: The burden of sepsis-associated mortality in the United States from 1999 to 2005: an analysis of multiple-cause-of-death data. Crit Care 2009, 13:R28.

2. Dellinger RP, Levy MM, Carlet JM, Bion J, Parker MM, Jaeschke R, Reinhart K, Angus DC, Brun-Buisson C, Beale R, et al: Surviving Sepsis Campaign: international guidelines for management of severe sepsis and septic shock: 2008. Crit Care Med 2008, 36:296-327.

3. Ospina-Tascon G, Neves AP, Occhipinti G, Donadello K, Buchele G, Simion D, Chierego ML, Silva TO, Fonseca A, Vincent JL, et al: Effects of fluids on microvascular perfusion in patients with severe sepsis. Intensive Care Med 2010, 36:949-955.

4. Davis JS, Darcy CJ, Yeo TW, Jones C, MCNeil YR, Stephens DP, Celermajer DS, Anstey NM: Asymmetric dimethylarginine, endothelial nitric oxide bioavailability and mortality in sepsis. PloS One 2011, 6:e17260.

5. Engelberger RP, Pittet YK, Henry H, Delodder F, Hayoz D, Chiolero RL, Waeber B, Liaudet L, Berger MM, Feihl F: Acute endotoxemia inhibits microvascular nitric oxide-dependent vasodilation in humans. Shock 2011, 35:28-34.

P67

Molecular diagnosis of severe bacterial sepsis in children

M Merisescu*, M Luminos, G Jugulete, D Florea, A Streinu-Cercel

National Institute of Infectious Diseases, Bucharest, Romania

Critical Care 2012, 16(Suppl 3):P67

Background: Bacterial sepsis is a very severe infection, potentially lethal, that requires an etiologic diagnosis and fairly complex treatment, quickly set. Plex-ID is the newest method in etiologic diagnoses of bacterial infections. It can detect bacterial DNA found in various pathological products: blood, CSF, joint fluid, pleural liquid.

Methods: We conducted a 6-month study from January to June 2012 on children admitted to our pediatric ICU of the National Institute of Infectious Diseases for severe forms of bacterial sepsis. In these children we monitored the following parameters: sex, age and origin (nosocomial or community-acquired infection). Positive diagnosis of sepsis was established on clinical and laboratory criteria (hemocultures, CSF cultures). As well as classical cultures, we found causal agents through the newest modern technique - Plex-ID. We watched for correlation of data obtained by classical methods versus molecular methods and the clinical and biological evolution of patients under treatment

Results: In the 6 months of study, 21 children met the clinical and biological criteria for bacterial sepsis. Seventy-six percent of patients came from other hospitals, the reason for which we consider that the etiology is most probably nosocomial pathogens. Sex distribution was approximately equal boys and girls, and in terms of distribution by age group prevailed in children aged 0 to 3 years $(80 \%)$. We obtained 15 positive results $(71 \%)$ by molecular methods that were correlated with conventional methods of diagnosis. The seat in the distribution of case etiology was as follows: Niessiera meningitides (four cases), Streptococcus pneumoniae (two cases), Staphylococcus spp. (four cases), Klebsiella pneumoniae (one case), Acinetobacter baumannii (one case), Pseudomonas aeruginosa (one case), Propionibacterium acnes (one case), and Haemophilus influenzae (one case).

Conclusion: Bacterial sepsis in children is a serious condition resulting in four deaths (19\%) with septic shock and multiple organ failure in our study. The patients require rapid etiologic diagnosis and establishment of appropriate emergency treatment. Plex-ID is an effective and rapid diagnosis method, identifying casual agent in $71 \%$ of cases.

The major advantage of this new method is the possibility to establish the etiological diagnosis in the early hours of patient admission. Also the speed and specificity of the modern diagnosis methods increase the survival index of the hospitalized patients with severe bacterial sepsis.

P68

Insulin exerts anti-inflammatory effects through reduction of IKK/I $\kappa \mathrm{B} /$

$\mathrm{NF}-\kappa \mathrm{B}$ pathway activation in septic rats

F Mittestainer*, B de Melo Carvalho, K Lima Calisto, D Guadagnini,

M Jose Abdalla Saad

University of Campinas, Brazil

Critical Care 2012, 16(Suppl 3):P68

Background: During the onset of sepsis, the inflammatory system becomes hyperactive, leading to an overproduction of proinflammatory mediators, which contribute to septic shock, multiple organ failure, and death. Animal studies indicate that insulin may have direct antiinflammatory effects, independent of its effect on hyperglycemia. However, the mechanism by which insulin reduces inflammation in the absence of hyperglycemia is unknown. The aim of the present study was to investigate of the molecular mechanisms of action and response of insulin administration in relation to its anti-inflammatory effect in septic animals. We analyzed the modulation of $I \mathrm{KK} / \mathrm{I} \kappa \mathrm{B} / \mathrm{NF}-\kappa \mathrm{B}$ pathways and insulin signaling pathway (IR, IRS-1 and Akt) and the expression of NF- $\kappa$ B p65 DNA binding in liver, muscle and adipose tissue in septic animals.

Methods: For the experiment, diffuse sepsis was induced by cecal ligation and puncture surgery in male adult Wistar rats. The septic animals were randomly divided into four different groups. Three of them were submitted to insulin injection in the portal vein and were stimulated for 1, 3 and 5 minutes, and the other group (control) was not stimulated (saline). After the stimulation time, insulin sensitive tissues were extracted and the expression and phosphorylation of the proteins were analyzed through western blot. NF- $\kappa \mathrm{B}$ p 65 activation was determined in nuclear extracts from muscle, liver and adipose tissue from septic rats by ELISA.

Results: In a time-course experiment we observed an increase in insulininduced phosphorylation of IR- $\beta$, IRS1 tyrosine phosphorylation, and Akt serine phosphorylation that reached a peak at 3 minutes post injection in muscle, liver and adipose tissue in septic animals. In parallel, there was a reduction in the phosphorylation of IKK in the time 1 and 3 minutes after stimulation by insulin returned to basal levels at 5 minutes. There was also a reduction in the phosphorylation of $I \kappa \mathrm{B}$ in the time 3 and 5 minutes after insulin injection. The DNA binding of NF- $\kappa \mathrm{B}$ p65 in the cell nucleus showed a reduction in expression levels of $\mathrm{NF}-\kappa \mathrm{B}$ at all times investigated, in accordance with the reduction of IKK and $I \kappa B$ phosphorylation after insulin injection in liver, muscle and adipose tissue of septic animals.

Conclusion: In summary, we report evidence indicating that the acute infusion of insulin activated the PI3K-Akt pathway and reduced IKK/I $\kappa \mathrm{B} /$ $\mathrm{NF}-\kappa \mathrm{B}$ indicate an important molecular mechanism to the anti-inflammatory effect of the hormone in liver, muscle and adipose tissue of septic animals. This helps explain a correlation between activation of insulin signaling and its effect on the inflammatory pathway.

\section{P69}

Severe sepsis with multiple organ dysfunctions caused by

Pseudomonas aeruginosa in an immunocompetent child

G Jugulete*, M Luminos, A Visan, A Draganescu, M Merisescu, M Vasile, A Nica Institute of Infectious Diseases, Bucharest, Romania

Critical Care 2012, 16(Suppl 3):P69

Introduction: Pseudomonas aeruginosa is a Gram-negative bacillus, which can cause severe infections especially in immunocompromised patients. Most infections caused by this germ are nosocomial because of increased antibiotic and antiseptic resistance but in rare cases we talk about community infections. Severe infection in immunocompetent patients caused by this microbe is extremely rare. The mortality rate in sepsis caused by $P$. aeruginosa is very high (up to $60 \%$ ).

Methods: We present the case of a 2-year-old boy admitted to our clinic for fever, diarrhea, impaired general condition, vesiculobullous and 
petechial exanthema. From the child's medical history we would like to mention: untreated perianal fissure and excised warts on terminal phalanges of his third finger of the left hand, big toe and the fifth toe of his left leg as the overgrowth and necrosis at the site of excision. Shortly after admission the patient presents an episode of acute respiratory failure followed by apnea and he is tracheal intubated and mechanically ventilated.

Results: During admission the general condition remains serious, the patient remains sedated and respiratory assisted with petechial exanthema, with hypotension, oligo-anuria, anal fissure, and necrotic lesions in the distal finger phalanges. Biological: severe pancytopenia $(\mathrm{WBC}=700 / \mathrm{mmc}, \mathrm{Hb}=8.2 \mathrm{~g} / \mathrm{dl}$, platelets $=34.000 / \mathrm{mmc}$ ), coagulation disorders, nitrogen retention syndrome, hypoalbuminemia, hepatic cytolysis syndrome, procalcitonin and inflammatory tests intensely positive. On smears made from the skin lesions, Gram-negative bacilli were visualized. The cultures of skin lesions were suggestive for $P$. aeruginosa, confirmed after 48 hours by blood culture identification and PCR determination (Plex-ID). We established comprehensive treatment: antibiotic (meropenem, ciprofloxacin, linezolid), antifungal, inotropic drugs (dobutamine, norepinephrine), ENP (rebalance acid-base and electrolyte), human immunoglobulin, blood and blood products transfusions and Neupogen. The evolution was favorable to the resumption of diuresis after 24 hours, increasing white blood cells and the lesions diminished.

Conclusion: Positive diagnosis of severe sepsis was based on clinical and laboratory findings. We have established the likely starting point of the patient's severe condition to be the multiple skin lesions and untreated anal fissure. Although the patient was immunocompetent, he developed a severe form of sepsis with septic shock caused by $P$. aeruginosa hardly responsive to treatment. We believe that the patient's favorable development was due to this germ's increased sensitivity to antibiotics, most probably community acquired.

P70

Effects of sesamol against acute kidney injury in cecal-ligation-andpuncture-treated rats

$S$ Chien $^{1 *}, Y L^{2} i^{2}, M L^{2}{ }^{2}$

${ }^{1}$ Tainan University of Technology, Tainan, Taiwan; ${ }^{2}$ National Cheng Kung University, Tainan, Taiwan

Critical Care 2012, 16(Suppl 3):P70

Background: Polymicrobial infection is associated with systemic inflammatory response, which is involved in the pathogenesis and development of acute kidney injury (AKI) [1,2]. In this study, we examined the effect of sesamol against AKI in cecal-ligation-and-puncture (CLP)treated rats.

Methods: Rats were given two subcutaneous doses of sesamol (10 mg/kg) 0 and 6 hours after CLP. Serum and kidney tissue were sampled 12 hours after CLP. Renal function and proinflammatory mediators, such as IL-1 $\beta$,
IL-6, and nitrite production were detected. Systemic oxidative stress was assessed by determining nitric oxide, superoxide anion, and xanthine oxidase activities. In addition, inducible nitric oxide synthase (iNOS) expression was also assessed in leukocytes from rats with AKI.

Results: The levels of serum BUN, CRE, IL-1 $\beta$, IL-6, nitrite, iNOS expression, superoxide anion, and xanthine oxidase activity were significantly higher in rats after CLP. Sesamol significantly inhibited all parameters in CLP-treated rats.

Conclusion: Sesamol attenuated AKI by inhibiting neutrophil-initiated systemic inflammation and oxidative stress in CLP-treated rats.

Acknowledgements: This study was supported by grants from the National Science Council, Taiwan.

References

1. Leithead JA, Ferguson JW, Bates CM, Davidson JS, Lee A, Bathgate AJ, Hayes $P C$, Simpson $\mathrm{KJ}$ : The systemic inflammatory response syndrome is predictive of renal dysfunction in patients with non-paracetamolinduced acute liver failure. Gut 2009, 58:443-449.

2. Li YH, Hsu DZ, Liu MY: Sesamol attenuates systemic inflammationassociated acute kidney injury in polymicrobial infectious rats. Science Against Microbial Pathogens: Communicating Current Research and Technological Advances Badajoz, Spain: Formatex Research Center: MendezVilas A 2011, 504-510.

P71

Noradrenergic neurons regulate the egress and trafficking of splenic monocytes and influence mortality during Gram-negative infection in mice

EJ Seeley ${ }^{1 *}$, S Barry ${ }^{2}, M^{\prime}$ Matthay ${ }^{1}$, PJ Wolters ${ }^{1}$

${ }^{1}$ University of California San Francisco, CA, USA; ${ }^{2}$ Stanford University, Palo Alto, CA, USA

Critical Care 2012, 16(Suppl 3):P71

Background: Neurotransmitters derived from the autonomic nervous system can regulate inflammatory cytokine secretion. This has been extensively studied in the parasympathetic nervous system, where acetylcholine regulates the secretion of TNF from splenic macrophages during LPS-induced inflammation. However, the role of noradrenergic neurons during mouse models of infection has not been characterized. The goal of these experiments was to study the influence of noradrenergic neurons on the immune response during Gram-negative septic peritonitis in mice.

Methods: Peripheral noradrenergic nerves were ablated using 6hydroxydopamine (6-OHDA), a commonly employed method for studying noradrenergic neurons. Four days later, septic peritonitis was induced by i.p. injection of 150 CFU Klebsiella pneumoniae. Survival, serum and peritoneal bacterial loads, inflammatory cytokine production and leukocyte recruitment were studied at multiple time points after infection. To assess the importance of the NE containing splenic nerve, survival experiments on splenectomized mice with or without 6-OHDA treatment were performed.

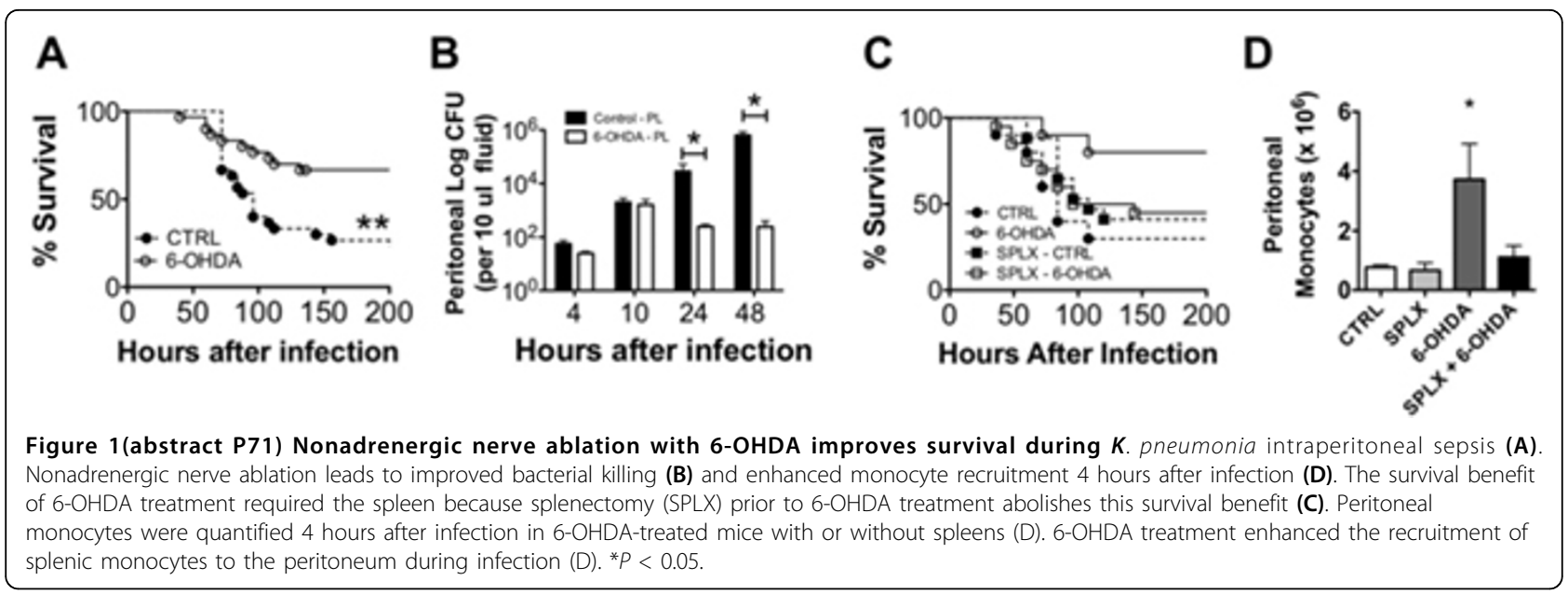


Results: Ablation of noradrenergic nerves improved survival following K. pneumoniae septic peritonitis (Figure 1A). Mice in which noradrenergic nerves had been ablated showed a more robust immune response 4 hours after infection with higher systemic IL-6, higher intraperitoneal MCP-1 and a fourfold increase in monocyte recruitment into the peritoneum. Bacterial loads were lower 24 and 48 hours after infection in mice in which noradrenergic nerves had been ablated (Figure 1B). Four hours after infection, 6-OHDA-treated mice recruited more inflammatory monocytes to the infected peritoneum (Figure 1D, CTRL vs. 6-OHDA). Splenectomy prior to noradrenergic nerve ablation abrogated the beneficial effects of 6-OHDA during infection (Figure $1 \mathrm{C}$ ) and reduced the recruitment of monocytes to the infected peritoneum (Figure 1D, 6-OHDA vs. SPLX + 6-OHDA).

Conclusion: These results suggest that splenic nerve-derived catecholamines regulate the egress of splenic monocytes during infection and that altering this pathway can alter survival during septic peritonitis in mice. These data highlight the emerging role of splenic monocytes during inflammation and infection [1] and add to the body of evidence that the immunosuppressive effects of catecholamines can impair innate immune responses during infection [2]. These experiments may have important implications for patients receiving vasopressors in the ICU.

\section{References}

1. Swirski FK, Nahrendorf M, Etzrodt M, et al: Identification of splenic reservoir monocytes and their deployment to inflammatory sites. Science 2009, 325:612-616

2. Wong $C H$, Jenne $C N$, Lee $W Y$, Leger $C$, Kubes $P$ : Functional innervation of hepatic iNKT cells is immunosuppressive following stroke. Science 2011, 334:101-105.

\section{P72}

Mannose-binding lectin deficiency and NOD2 mutations do not predispose to Staphylococcus aureus bloodstream infections but may influence outcome

M Osthoff ${ }^{1 *}, \mathrm{HM}$ Au Yong ${ }^{1}$, MM Dean $^{2}$, D Eisen

${ }^{1}$ Royal Melbourne Hospital, Victorian Infectious Diseases Service, Parkville, Australia; ${ }^{2}$ Australian Red Cross Blood Service, Brisbane, Australia Critical Care 2012, 16(Suppl 3):P72

Background: Staphylococcus aureus is a major cause of bloodstream infections (BSI), and is associated with a higher morbidity and mortality compared with other BSI pathogens. Innate pattern recognition receptors like mannose-binding lectin (MBL) of the complement system and NOD2 (nucleotide-binding oligomerization domain-containing protein 2), an intracellular sensor for a variety of pathogens, have been shown to be crucially involved in the immune response against $S$. aureus in knockout animal models $[1,2]$, but human data are lacking. Low MBL levels and NOD2 mutations can be found in up to $30 \%$ and $10 \%$ in the general population, respectively $[3,4]$. This study aimed to investigate whether MBL deficiency and NOD2 mutations predispose to and influence the severity of S. aureus BSI.

Methods: A matched case-control study was undertaken involving 70 patients with $S$. aureus BSI and 70 age-matched and sex-matched hospitalized controls recruited prospectively at two major tertiary hospitals. Participant blood samples were analyzed for MBL levels by mannan-binding ELISA and for four MBL2 and three NOD2 polymorphisms by real-time PCR. Clinical and microbiological data were reviewed. $M B L$ deficiency was defined as functional MBL level $\leq 0.1 \mu \mathrm{g} / \mathrm{ml}$. Univariate and multivariate conditional logistic regression was used to investigate the risk of BSI in matched controls and cases.

Results: S. aureus BSI were nosocomially acquired (60\%) and intravenous catheter associated (50\%) in the majority of cases with an in-hospital mortality of $10 \%$. After adjusting for diabetes, immunosuppression, chronic kidney disease and long-term intravenous catheters, MBL deficiency was found less frequently in cases than controls ( $8.6 \%$ vs. $20 \%$, $\mathrm{OR}=0.38, P=0.07)$ as were low-producing $\mathrm{MBL}$ genotypes $(11 \%$ vs. $23 \%$, OR $=0.37, P=0.05)$, whereas NOD2 polymorphisms were similarly distributed $(14 \%$ vs. $10 \%, P=0.4)$. In line with $M B L 2$ genotypic results, $M B L$ levels were significantly higher in cases than in controls (adjusted $\mathrm{OR}=1.35$ per $1 \mu \mathrm{g} / \mathrm{ml}$ increase, $P=0.002$; Figure 1). Cases with NOD2 polymorphisms had less severe disease manifestations as shown by a lower SOFA score (mean 2.1 vs. 4.4, $P=0.04$ ) and a reduced rate of

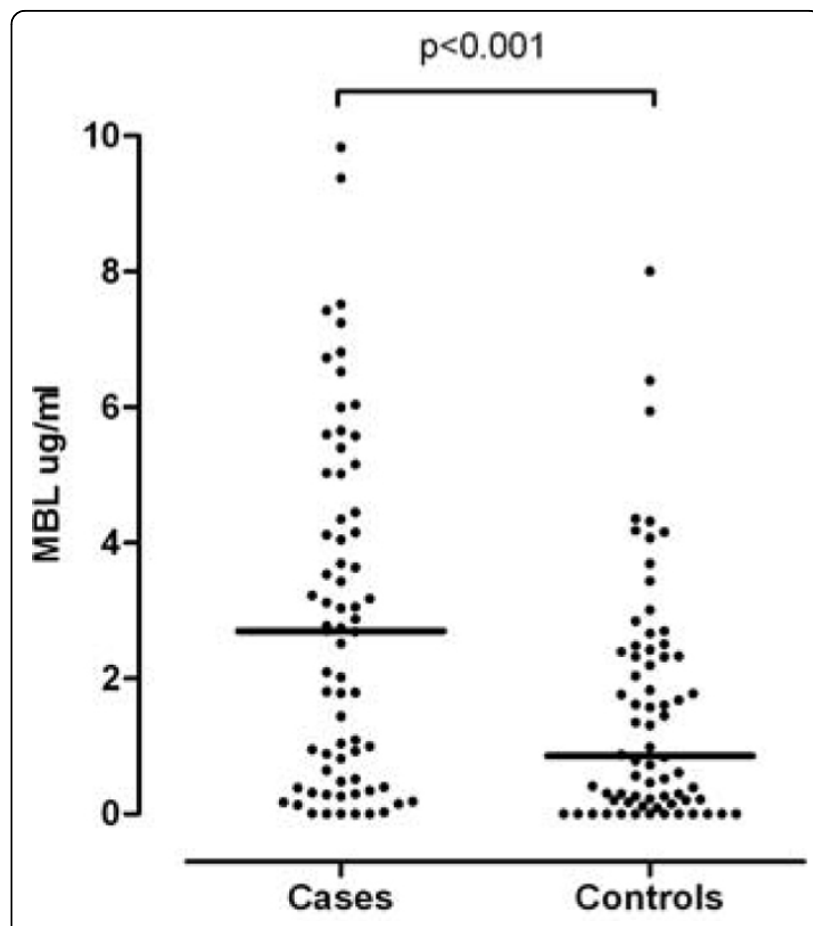

Figure 1(abstract P72) Differences in MBL serum concentrations in cases (S. aureus BSI) and controls. Horizontal lines represent medians.

multiorgan dysfunction and death (40\% vs. $60 \%, P=0.06)$, whereas $\mathrm{MBL}$ deficiency had no influence on the severity of $S$. aureus BSI.

Conclusion: Neither MBL deficiency nor NOD2 polymorphisms were associated with an increased risk of $S$. aureus BSI. In fact, contrary to experimental data, MBL deficiency seemed to confer protection in acquiring S. aureus BSI, and NOD2 mutations were less frequently associated with multiorgan dysfunction in this matched case-control study.

\section{References}

1. Deshmukh HS, Hamburger JB, Ahn SH, McCafferty DG, Yang SR, Fowler VG $\mathrm{Jr}$ : Critical role of NOD2 in regulating the immune response to Staphylococcus aureus. Infect Immun 2009, 77:1376-1382.

2. Ip WK, Takahashi K, Moore KJ, Stuart LM, Ezekowitz RA: Mannose-binding lectin enhances Toll-like receptors 2 and 6 signaling from the phagosome. J Exp Med 2008, 205:169-181.

3. Eisen DP, Dean MM, Boermeester MA, Fidler KJ, Gordon AC, Kronborg G, Kun JF, Lau YL, Payeras A, Valdimarsson $\mathrm{H}$, et al: Low serum mannosebinding lectin level increases the risk of death due to pneumococcal infection. Clin Infect Dis 2008, 47:510-516.

4. Hugot JP, Zaccaria I, Cavanaugh J, Yang H, Vermeire S, Lappalainen M, Schreiber S, Annese V, Jewell DP, Fowler EV, et al: Prevalence of CARD15/ NOD2 mutations in Caucasian healthy people. Am J Gastroenterol 2007, 102:1259-1267.

P73

Homogeneity versus diversity: inhibition of plasma PAI-1 in murine sepsis proved lethal in homogeneous cohorts but not in all-inclusive populations

P Raeven ${ }^{1 *}$, KM Weixelbaumer ${ }^{1}$, S Drechsler ${ }^{1}$, A Klotz ${ }^{1}$, M Jafarmadar ${ }^{1}$,

A Khadem ${ }^{1}$, H Redl $^{1}$, S Bahrami ${ }^{1}$, PJ Declerck ${ }^{2}$, MF Osuchowski ${ }^{1}$

${ }^{1}$ Ludwig Boltzmann Institute for Experimental and Clinical Traumatology,

AUVA Trauma Research Center, Vienna, Austria; ${ }^{2}$ Laboratory for

Pharmaceutical Biology, KU Leuven, Belgium

Critical Care 2012, 16(Suppl 3):P73

Background: Plasminogen activator inhibitor type 1 (PAI-1) inhibits fibrinolysis and its plasma increases correlate with exacerbated sepsis mortality. However, its exact role in abdominal sepsis outcomes 
is unclear. We aimed to test the effects of fibrinolysis restoration upon survival during acute polymicrobial sepsis using a custom-developed antiPAl-1 monoclonal antibody. We specifically studied whether the effects of the treatment vary between all-inclusive populations and homogeneous cohorts stratified based on the risk of death.

Methods: Mice underwent cecal ligation and puncture (CLP) and received a single intravenous injection of either anti-PAl-1 (10 mg/kg; MA-MP6H6) or control antibody (MA-Control). Three approaches were used: approach I, co-treatment (CLP and MA-MP6H6 administered at 0 hours) without stratification; approach II, post treatment (MA-MP6H6 at 18 hours post CLP) without stratification; and approach III, post treatment (MA-MP6H6 at 30 hours) with prospective stratification. Then $20 \mu$ blood (facial vein) was sampled daily from all mice until day 5 and survival was followed for 28 days. In approach I, a group of mice was sampled at 6 hours and sacrificed at 24 hours to test the MA-MP6H6 efficacy. In approach III, mice were stratified into either predicted to survive (P-SUR) or die (P-DIE) based on circulating IL-6 measured at 24 hours post CLP.

Results: MA-MP6H6 co-treatment blocked 60 to $100 \%$ of active plasma PAI-1 and restored fibrinolysis (fibrin plate assay) in septic animals at 24 hours post application. Without stratification, MA-MP6H6 co-treatment and 18-hour (also 30-hour) post treatment neither conferred benefit nor was detrimental. Post treatment with MA-MP6H6 after prospective IL-6 stratification at $14 \mathrm{ng} / \mathrm{ml}$ cutoff ( $48 \%$ sensitivity for P-DIE and $100 \%$ specificity for P-SUR) showed $15 \%$ exacerbation of mortality $(P>0.05)$. Cutoff readjustment to $3.3 \mathrm{ng} / \mathrm{ml}$ maximized P-DIE cohort homogeneity (82\% sensitivity; $100 \%$ specificity) revealing harmful MA-MP6H6 effects: in P-SUR, no MA-Control-treated mice ever died, but $30 \%$ of MA-MP6H6treated did $(P<0.05)$. In P-DIE, all MA-MP6H6-treated mice died within
72 hours, while MA-Control mice lived up to 8 days $(P<0.05)$. In cotreatment, MA-MP6H6 increased circulating IL- 6 and $\mathrm{CXCL}-1 / \mathrm{KC}$ by threefold and twofold at 24 hours (MCP-1, MIP-1 $\alpha$, IL-1 $\beta$, IL-10 and TNF $\alpha$ unaffected), but had no effect in post treatment (24 to 96 hours). Regardless of the treatment approach, MA-MP6H6 did not significantly alter hematological and/or organ function parameters.

Conclusion: Restoration of fibrinolysis by neutralization of PAI-1 in acute sepsis lacks benefit and may prove fatal. Remarkably, the detrimental effect of MA-MP6H6 became apparent only after a maximally homogeneous separation of high/low risk-of-death cohorts. A prospective separation of septic patients into various well-defined, homogenous cohorts is critical in revealing negative side effects of tested anti-sepsis therapeutics.

Acknowledgements: Supported by WWTF Grant LS07-065.

P74

Effect of heparin during extracorporeal detoxification in the severity of thrombocytopenia in patients with severe sepsis

W Kulabukhov*, AG Chizhov, AN Kudryavtsev

Vishnevsky Institute of Surgery, Burn Center, Moscow, Russia

Critical Care 2012, 16(Suppl 3):P74

Background: Disseminated intravascular coagulation plays an important role in the pathogenesis of severe sepsis. Current treatment of sepsis is not possible without the use of extracorporeal blood purification methods; the use of heparin is one of the measures of stabilization circuit. Intravenous administration of heparin can cause a decrease in platelet count. A reliable

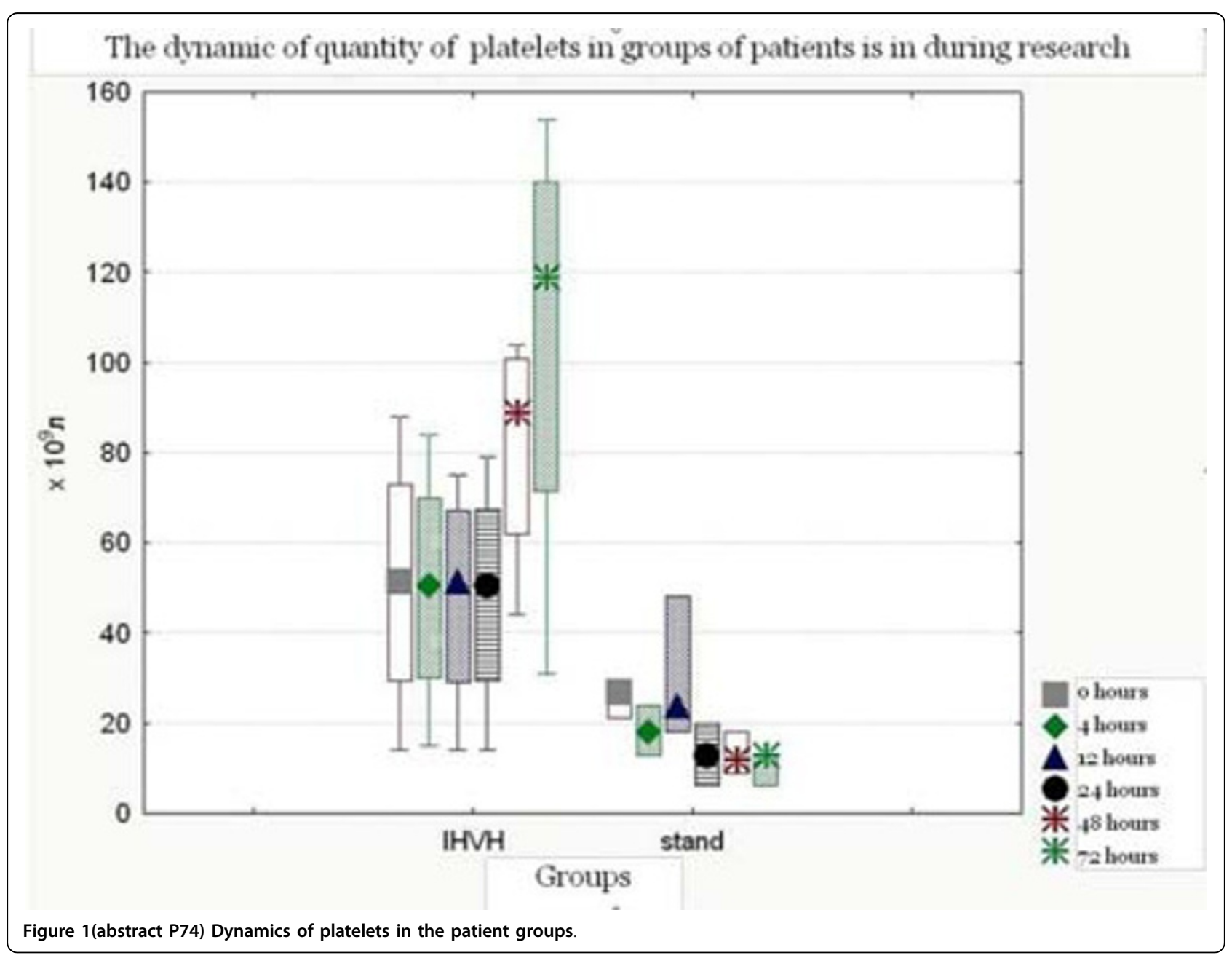


technique for differential diagnostics of heparin-induced thrombocytopenia is using $4 \mathrm{~T}$ scales [1].

Methods: An observational retrospective cohort study of 106 patients in the period from 2010 to 2011. The aim of the study was to determine the effect of heparin on the severity of clinical signs of heparin-induced thrombocytopenia in patients with severe sepsis. Inclusion criteria were the presence of severe sepsis in a patient (Surviving Sepsis Campaign 2008), the need for extracorporeal blood purification, and thrombocytopenia. The mean age of all the patients was $54 \pm 15.91$, mean SOFA score was $7.4 \pm 0.9$ ( 2 to 13). Reduced platelet counts were observed in 23 patients. Patients at high risk for heparin-induced thrombocytopenia type II (4 to 5 points for the 4T scale) in the study were not included. Ten of the patients $(9.4 \%)$ conducted a session intermittent high-volume hemofiltration (group IHVH) within 4 hours, c volume replacement of $100 \mathrm{ml} / \mathrm{kg} /$ hour. Eleven patients (10.4\%) did not receive extracorporeal blood purification (group Stand), because of uncontrolled bleeding. All patients received therapy according to Surviving Sepsis Campaign 2008. The standard dose of heparin was 8 to 15 units $/ \mathrm{kg} /$ hour.

Results: In group IHVH the increase in platelet count at the end of the second day treatment was $105.75 \pm 52.8 \times 10^{9} /$ (Figure 1 ). This level is significantly different from the number of platelets in group Stand to the same phase of the study $\left(10.7 \pm 4.0 \times 10^{9} / \mathrm{l}\right)$. During treatment in the groups studied there were no thromboses. The 28-day period mortality in group IHVH was 20\% (two patients), and in group Stand was 9\% (one patient).

Conclusion: The use of heparin, including the extracorporeal blood purification, can be safe with heparin-induced thrombocytopenia type I in patients with severe sepsis.

\section{Reference}

1. Warkentin TE, Heddle NM: Laboratory diagnosis of immune heparininduced thrombocytopenia. Curr Hematol Rep 2003, 2:148-157.
P75

Estimation of efficacy early selective LPS sorption in patients with septic shock

W Kulabukhov*, AN Kudryavtsev, AG Chizhov

Vishnevsky Institute Of Surgery, Burn Center, Moscow, Russia Critical Care 2012, 16(Suppl 3):P75

Background: Recent improvements in the treatment of severe Gramnegative sepsis have not resulted in a substantial decrease in mortality. LPS, originating from Gram-negative bacteria, is a major mediator in the development of septic shock [1]. Decreasing the load of LPS in these patients may be beneficial for these patients. The Alteco LPS Adsorber ${ }^{\bullet}$ selectively binds the lipid A moiety of LPS. Positive effects of the adsorber have been reported [2-4].

Methods: Nineteen patients with Gram-negative sepsis were treated with the Alteco LPS Adsorber ${ }^{\oplus}$, in addition to standard therapy according to SSC Guidelines 2008. All patients needed inotropic support and mechanical lung ventilation. The mean APACHE II score at the start of treatment was $25.9 \pm 1.8$. The time to initiating the perfusion varied between 1.7 and 8.6 hours after the diagnosis of septic shock. The treatment lasted 120 minutes and was repeated after 24 hours. The SOFA score, oxygenation index $\left(\mathrm{PaO}_{2} / \mathrm{FiO}_{2}\right)$ and the dose of dopamine was noted before and 48 hours after the treatment.

Results: At baseline, the severity of MODS was SOFA score $9.2 \pm 2.8$. At 48 hours the mean score SOFA was $4.3 \pm 2.7$, at the expense of an increase in the index of oxygenation from $128.6 \pm 36.2$ to $253.5 \pm 44.8$. and a decrease in doses of inotropic support (dopamine) from $17.1 \pm$ $1.8 \mathrm{mkg} / \mathrm{kg} / \mathrm{minute}$ to $4.2 \pm 1.8 \mathrm{mkg} / \mathrm{kg} / \mathrm{minute}$. We found an inverse correlation between the time to initiate treatment with the adsorbtion and the decrease in SOFA score $(R=-0.69 ; P<0.1)$ (Figure 1). The degree

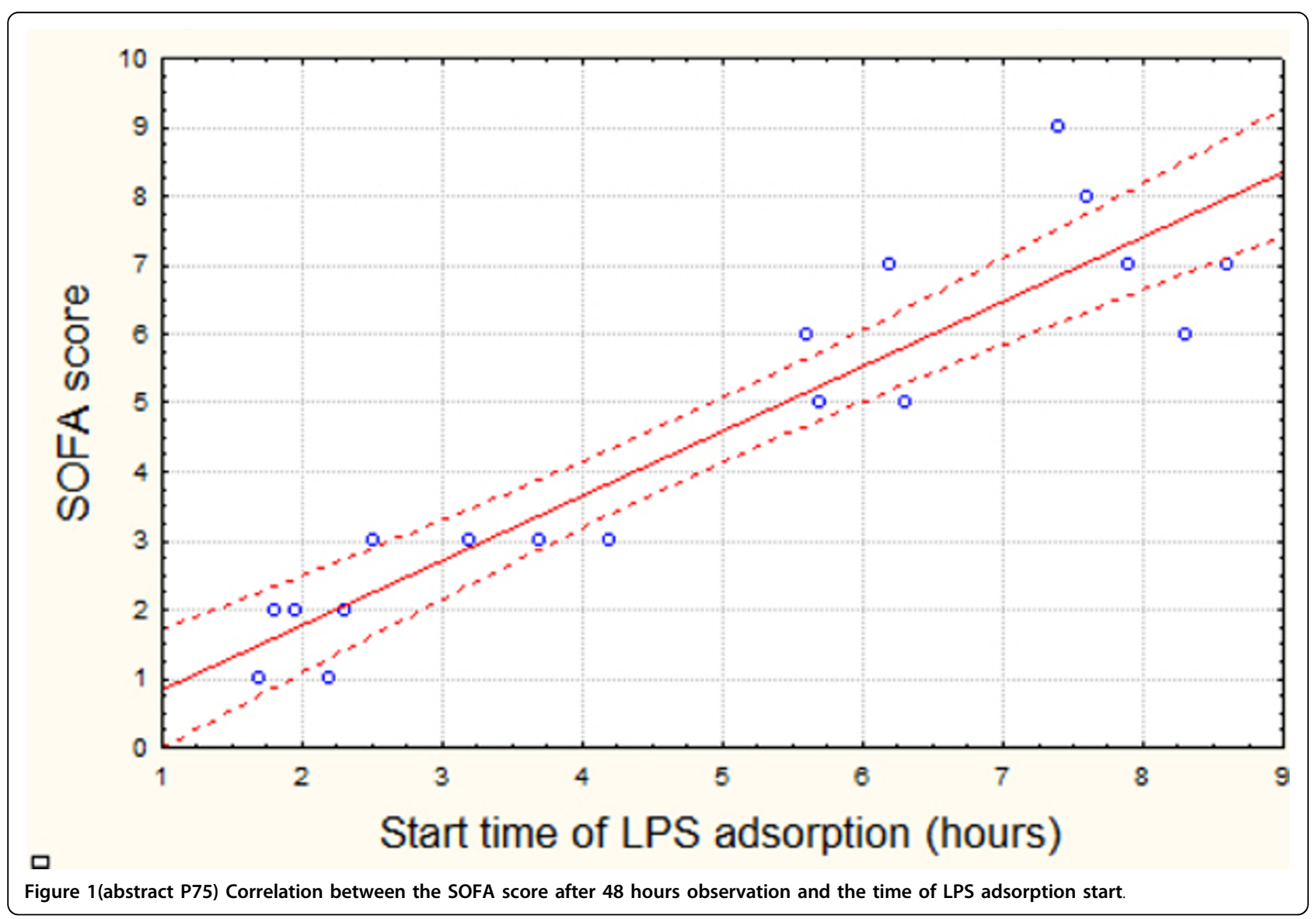




\section{Figure 2. Reducing the degree of MODS as compared with the starting time of LPS- adsorption ( $\%$ of baseline)}

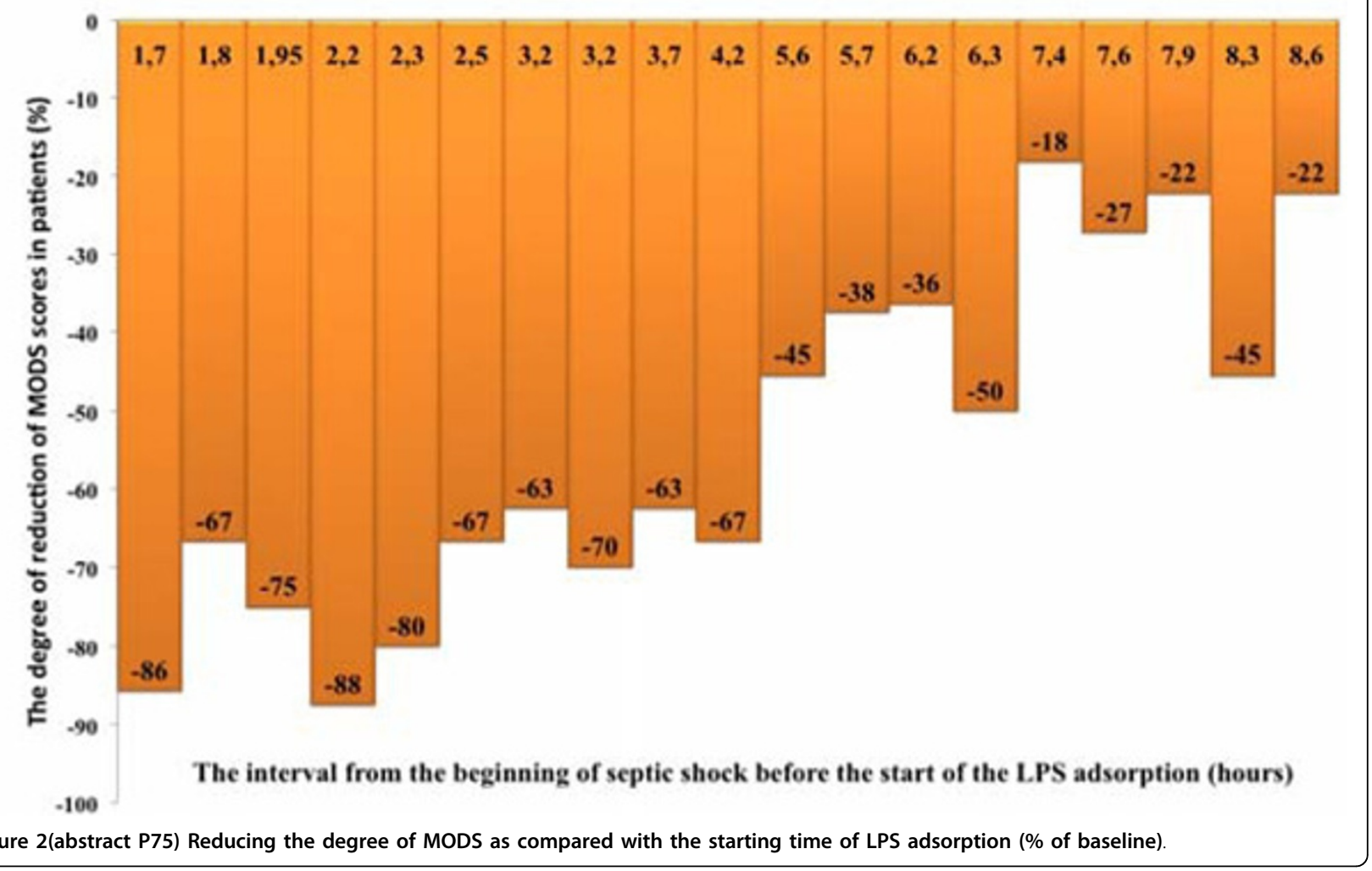

of reduction of MODS depending on time of initiation of therapy is shown in Figure 2 . The 28 -day mortality was $26.3 \%$ in this patient group. There were no adverse events related to use of the adsorber.

Conclusion: Since LPS is a major mediator in Gram-negative sepsis, there is a rationale for rapid removal of LPS from patients with sepsis. Early initiation of LPS adsorption is most effective for reducing the signs of MODS. Our experiences with the use of Alteco LPS Adsorber ${ }^{\oplus}$ are promising as shown by our data above and are in support of previous findings [2-4]. The results are limited by the lack of controls; however, we believe that further randomized controlled clinical studies using this therapy are warranted, more so in view of the fact that no specific treatment against septic shock is available.

References

1. Marshall JC, et al: Diagnostic and prognostic implications of endotoxemia in critical illness: results of the MEDIC study. J Infect Dis 2004, 190:527-534

2. Kulabukhov $\mathrm{V}$ : Use of novel technique for adsorption of

lipopolysaccharide in the combined treatment of patients with severe organ negative sepsis [abstract 78]. Sepsis 2008; An International Sepsis Symposium; Paris. France.

3. Kulabukhov V, Chizhov A, Kleuzovich A, Kudryavtsev A: Clinical effects of adsorption of lipopolysaccharide in the treatment of Gram-negative severe sepsis. Crit Care 2010, 14(Suppl 2):P28.

4. Ala-Kokko TI, Laurila J, Koskenkari J: A new endotoxin adsorber in septic shock: observational case series. Blood Purif 2011, 32:303-309.

P76

Audit of the ward-based management of severe sepsis in a large teaching hospital

G Estebanez*, M Cole

Royal Liverpool and Broadgreen University Hospitals NHS Trust, Liverpool, UK Critical Care 2012, 16(Suppl 3):P76
Background: The Surviving Sepsis 6-hour bundle [1] was created to promote effective identification and management of patients with severe sepsis and septic shock. Efficient and timely implementation of the 6-hour bundle including early intravenous antibiotics has been shown to improve patient outcome [2]. We aimed to determine the efficiency of severe sepsis identification, implementation of the 6-hour bundle and overall management of ward-based patients.

Methods: During a 4-month period, ward-based patients with severe sepsis were identified by nurse practitioners and the critical care outreach team at a large teaching hospital in North West England. Analysis of patient medical records was performed to assess the efficiency and quality of care received compared with the gold standard sepsis 6-hour bundle. Promptness of doctor attendance and patient 30-day outcome were also analysed.

Results: Twenty-eight patients with severe sepsis were identified and analysed. Suspicion of sepsis was documented in medical records for all patients; however, the 6-hour sepsis bundle was completed in only one case. Appropriate i.v. antibiotics were prescribed to all patients. Median duration from the documented onset of severe sepsis (time 0 ) until review by a doctor was 90 minutes ( 0 to 19 hours). The median Modified Early Warning Score (MEWS), determined from patients' vital signs, at documented onset of severe sepsis was 3.5 (1 to 9) with a subsequent increase of median MEWS to 4 by the time of doctors' first attendance. The 30-day patient outcome showed 13 patients discharged home and seven patients deceased. Comparison of deceased patients with patients who were discharged alive (see Table 1) demonstrated an increased median duration from time 0 until doctor attendance in the deceased group. However, interestingly, timely antibiotics (given within 1 hour of patient assessment by a doctor) were administered with greater frequency in the deceased patient group.

Conclusion: Despite the widespread recognition of patients with severe sepsis, underperformance of the 6-hour bundle remains a major factor in 


\section{Table 1(abstract P76) Characteristics and management data for patients with severe sepsis comparing patients who} were deceased with patients discharged home at $\mathbf{3 0}$ days

\begin{tabular}{|c|c|c|}
\hline & $\begin{array}{l}\text { Patients discharged home at } 30 \text { days } \\
(n=13)\end{array}$ & $\begin{array}{l}\text { Patients deceased at } 30 \text { days } \\
(n=7)\end{array}$ \\
\hline Median age (years) & 77 & 68 \\
\hline Median time taken to be seen by doctor after time 0 (minutes) & 90 & 240 \\
\hline Median MEWS at time 0 & 3 & 3 \\
\hline Median MEWS at doctors' attendance & 3 & 4 \\
\hline Timely antibiotics given & $15 \%$ & $43 \%$ \\
\hline $\begin{array}{l}\text { Basic resuscitation undertaken (oxygen, i.v. fluids, urinary } \\
\text { catheter, } A B G \text { ) }\end{array}$ & $90 \%$ & $93 \%$ \\
\hline
\end{tabular}

Time $0=$ time of initial documented evidence of severe sepsis.

suboptimal management of ward-based patients. Increased delay from the onset of severe sepsis until doctor review is associated with increased risk of mortality despite better adherence to the 6-hour bundle. Further education of doctors and nursing staff, regarding the importance of the 6-hour sepsis bundle, in addition to the implementation of strategies to improve the early identification and timely review of ward-based patients with severe sepsis are recommended.

Acknowledgements: Olivia O'Gara and Jonathan Walker are contributing authors.

References

1. Dellinger RP, et al: Surviving Sepsis Campaign: international guidelines for management of severe sepsis and septic shock: 2008. Intensive Care Med 2008, 34:17-60.

2. Kumar $A$, et al: Duration of hypotension before initiation of effective antimicrobial therapy is the critical determinant of survival in human septic shock. Crit Care Med 2006, 34:1589-1596.

\section{P77}

Neutrophil CD64 as a diagnostic marker of sepsis in neonates: impact on clinical care

P Kingma ${ }^{1 *}$, E Hall $^{1}$, D Marmer ${ }^{2}$

${ }^{1}$ Cincinnati Children's Hospital Medical Center, The Perintal Institute,

Cincinnati, OH, USA; ${ }^{2}$ Cincinnati Children's Hospital Medical Center, Cancer

and Blood Diseases Institute Laboratory, Cincinnati, OH, USA

Critical Care 2012, 16(Suppl 3):P77

Background: Bacterial infections are a significant cause of morbidity and mortality in newborn infants. Successful treatment of neonatal infection depends on the early initiation of antibiotic therapy; however, unnecessary use of antibiotics increases bacterial resistance and has been associated with increased rates of necrotizing enterocolitis and death in premature infants. Unfortunately, the early clinical signs and symptoms of neonatal infection are often confused with other non-infectious conditions in premature infants such as apnea of prematurity and chronic lung disease. Neonatologists have traditionally relied on white blood cell counts (CBC) and bacterial cultures to help identify infected infants, but the $C B C$ is an unreliable marker of infection in the neonatal population and bacterial cultures are too slow to be useful in the immediate evaluation of an infant. Methods: Therefore, in order to overcome these obstacles and improve the identification of infected infants, we incorporated neutrophil CD64 levels into the infection evaluations in our newborn ICU and evaluated the impact of this change on clinical care.

Results: A total of 405 evaluations were performed in 268 infants (ages 1 to 293 days) from 2005 to 2009 . Twenty-nine infants had culture-positive sepsis. The sensitivity and negative predictive value of the neutrophil CD64 assay was consistently $\geq 90 \%$ in identifying infected and non-infected infants. In fact, during the 3-year analysis period only three infants were identified with positive blood cultures that had a normal CD64 index. Two of these infants had blood cultures that were positive for Staphylococcus epidermidis and one had a positive blood culture that was obtained from a colonized central line. Although absolute CD64 levels did not correlate with severity of illness in our population (as determined by the need for ventilation $(P=0.87)$ or inotropic support $(P=0.90))$, relative decreasing CD64 levels did correlate well with the resolution of infection within a given infant. When compared with infants with an elevated CD64 level, a normal CD64level decreased unnecessary antibiotic exposure by 3.9 days. By comparison, in infants evaluated with the traditional methods that did not include a CD64 level, a normal CBC only decreased antibiotic exposure by 1 day when compared with infants with an abnormal CBC.

Conclusion: In summary, the clinical care that we provide our infants has improved with the use of neutrophil CD64 levels in our infectious evaluations.

P78

Pancreatic stone protein as a novel marker for neonatal sepsis L Schlapbach $^{1 *}$, R Graf ${ }^{2}$, A Woerner ${ }^{3}$, M Nelle $^{3}$, M Stocker $^{4}$, M Fontana $^{4}$ U Zimmermann-Baer ${ }^{5}$, D Glauser ${ }^{5}$, E Giannoni $^{6}$, T Roger $^{7}$, C Mueller $^{8}$

${ }^{1}$ Mater Children's Hospital, South Brisbane, Australia; ${ }^{2}$ Swiss HPB Center, University Hospital Zurich, Switzerland; ${ }^{3}$ University of Berne, Switzerland; ${ }^{4}$ Children's Hospital Lucerne, Switzerland; ${ }^{5}$ Clinic of Neonatology, Cantonal Hospital Winterthur, Switzerland; '6ervice of Neonatology, Centre Hospitalier Universitaire Vaudois and University of Lausanne, Switzerland;

${ }^{7}$ Infectious Diseases Service, Centre Hospitalier Universitaire Vaudois and University of Lausanne, Switzerland; ${ }^{8}$ Institute for Pathology, Universiy of Berne, Switzerland

Critical Care 2012, 16(Suppl 3):P78

Background: Early-onset sepsis (EOS) represents one of the main causes for ICU admission in newborns and therefore imposes a considerable burden on the healthcare system. Performance of traditional infection markers to diagnose EOS is poor and therefore insufficient to guide the decision to start or stop antibiotic treatment. While procalcitonin (PCT) has become an established sepsis marker in adults and children, sensitivity and specificity of PCT are only moderate in EOS due to the physiologic PCT increase during the first days of life. Pancreatic stone protein (PSP) is a promising sepsis marker in adults. This study investigated whether determining PSP improves diagnosis of EOS in comparison with other infection markers.

Methods: A prospective multicentre study including 137 infants $>34$ weeks gestational age admitted with suspected EOS. PSP, PCT, soluble human triggering receptor expressed on myeloid cells-1 (sTREM-1), macrophage migration inhibitory factor (MIF) and C-reactive protein (CRP) were measured at admission. Receiver-operating characteristics (ROC) curve analysis and multivariate logistic regression were performed. Bioscores were constructed using two, three and four sepsis markers.

Results: PSP was significantly higher in infected compared with uninfected infants (median 11.3 vs. $7.5 \mathrm{ng} / \mathrm{ml}, P=0.001$ ). The ROC area under the curve resulted at $0.69(95 \% \mathrm{Cl}=0.59$ to $0.80, P<0.001)$ for PSP, at 0.77 ( $95 \% \mathrm{Cl}=0.66$ to $0.87, P<0.001)$ for $\mathrm{PCT}$, at $0.66(95 \% \mathrm{Cl}=0.55$ to 0.77 , $P=0.006$ ) for CRP, at 0.62 ( 0.51 to $0.73, P=0.055$ ) for sTREM-1 and at 0.54 ( 0.41 to $0.67, P=0.54$ ) for MIF. In multivariate models, increased PSP levels showed the strongest association of all markers with EOS and PSP $>9 \mathrm{ng} / \mathrm{ml}$ independently of PCT predicted EOS (odds ratio $=26.4,95 \% \mathrm{Cl}=4.0$ to $172.5, P<0.001)$. Combining both markers significantly increased the ability to diagnose EOS $(P<0.001)$. The bioscore based on PSP and PCT performed best with an AUC of $0.83(95 \% \mathrm{Cl}=0.74$ to $0.93, P<0.001)$ and was superior 
to PCT or PSP alone. The combined PSP/PCT score had a NPV of $100 \%$ if both markers were below cutoff and a PPV of $71 \%$ if both were positive. Conclusion: In this prospective study, the diagnostic performance of PSP and PCT was far superior compared with traditional markers and a combination bioscore improved diagnosis of sepsis. Our findings suggest that PSP is a valuable biomarker in combination with PCT in EOS. To the best of our knowledge, this is the first study investigating PSP in neonatal sepsis.

P79

A standardized protocol for the multiplex PCR technique Septifast ${ }^{\oplus}$ Roche for neonatal samples with suspected sepsis

F Ortiz lbarra ${ }^{1 *}$, J Reyna ${ }^{2}$, P Treviño ${ }^{3}$, L Fernandez ${ }^{4}, G$ Lara $^{5}$, E Valenzuela', Y Morales ${ }^{4}$, A Limon 6 , A Ceballos ${ }^{7}$

${ }^{1}$ Laboratorios Diagnomol, Mexico; ${ }^{2}$ INSP, Mexico; ${ }^{3} \mathrm{HMI}$ SSNL, Monterrey,

Mexico; ${ }^{4}$ INPerlER, Mexico; ${ }^{5} \mathrm{HGO} 4$ IMSS, Mexico; ${ }^{6} \mathrm{HAE}$ Pemex Sur, Mexico;

${ }^{7} \mathrm{H}$ Dalinde, Mexico

Critical Care 2012, 16(Suppl 3):P79

Introduction: High morbidity and mortality rates are associated with sepsis in newborns, as well as low recovery rates, extended recovery times, and delayed culture times of blood culture. To surmount these parameters, new molecular techniques are required by the clinical laboratory. The LightCycler Septifast ${ }^{\oplus}$ technique, which identifies up to 25 microorganisms known to cause over $90 \%$ of admissions to ICUs due to infection, has proven its usefulness in adult patients in several countries. The objective was to standardize the Septifast ${ }^{\oplus}$ Roche technique for diagnostic use in newborns with suspected sepsis.

Methods: Eighty-six newborn samples with suspected sepsis (according to the NOSEP-1 scale) were included. We analyzed two blood samples per patient, the first one collected in an EDTA-anticoagulated tube ( 0.5 to $1.0 \mathrm{ml})$, and a second sample of $1 \mathrm{~cm}^{3}$ in a hemoculture tube for automated hemoculture procedure using BacT/ALERT ${ }^{\oplus}$ D (Biomerieux). Briefly, the whole sample was lysed with MagNA Lyser (Roche), and nucleic acid purification was performed with Septifast prep $\mathrm{M}^{\text {grade }}$ (Roche). DNA from Gram-negative, Gram-positive, and fungi was detected using multiplex LightCycler 2.0 real-time methodology with LightCycler SeptiFast $M^{\text {grade }}$ kit (Roche). Finally, results analysis was performed with Roche Septifast identification software (SIS).

Results: Of the 86 samples analyzed, 31 (36.04\%) were positive in at least one of the identification procedures. Thirteen samples $(15.11 \%)$ were rendered positive by hemoculture, and 27 (31.39\%) by Septifast ${ }^{\oplus}$ protocol. Fifty-seven samples $(66.20 \%)$ were negative by both analytic procedures, and a total of 31 pathogens (15 Gram-negative, $11 \mathrm{Gram}$-positive and five fungi species) were identified by Septifast ${ }^{\oplus}$ (four of them in combination) versus nine species recovered from hemocultures. The response time ranged from 6 to 24 hours by Septifast ${ }^{\oplus}$ procedure, compared with 4 to 7 days by hemoculture detection.

Conclusion: The use of the multiplex real-time PCR Septifast ${ }^{\oplus}$ technique allows one to detect a wider number of pathogenic species and a faster response time than hemoculture, with a small quantity of blood sample (1 ml).

\section{P80}

Toll-like receptor 4 in phagocytosis of Escherichia coli by endotoxinactivated human neutrophils in whole blood

I Prokhorenko*, S Zubova, D Kabanov, E Voloshina, S Grachev

Institute of Basic Biological Problems, Pushchino, Moscow Region, Russia Critical Care 2012, 16(Suppl 3):P80

Background: Endotoxic shock during infection by Gram-negative bacteria could frequently lead to mortality. Bacterial endotoxins (lipopolysaccharides (LPS)) play a major role in pathogenesis of Gram-negative sepsis. The infection after injury, burn or surgery could lead to accumulation of released endotoxins in the bloodstream. LPS are able to interact with specific receptors on the surface of target host cells (monocytes/macrophages, neutrophils, endothelial cells, and so forth). After interaction with LPS, phagocytes change their morphological and functional properties, namely receptor expression, synthesis of proinflammatory cytokines and phagocytic activity. In the LPS signaling pathway, Toll-like receptor 4 (TLR4, CD284) is the main cell surface molecule inducing activation of NF- $\kappa \mathrm{B}$-dependent genes. Recent reports suggest that TLRs are involved in phagocytosis as the assistance receptors of phagocytes helping them to recognize invading bacteria or their molecular patterns $[1,2]$. Intensity of phagocytosis is potentiated by prior neutrophil exposure to endotoxins [3]. TLRs ligation by endotoxin molecules induces adaptor protein MyD88-dependent signaling involving IL-1 receptor-associated kinase (IRAK) and p38 mitogen-activated protein kinase leading finally to upregulation of phagocytosis-inducing genes. The integrated collaboration between TLR4 and phagocytosis has been shown early [4]. As shown during phagocytosis, TLR4 was only integrated in this functional property of the cells but not involved as the primary receptor [5]. It is well known that appropriate cell response to LPS is

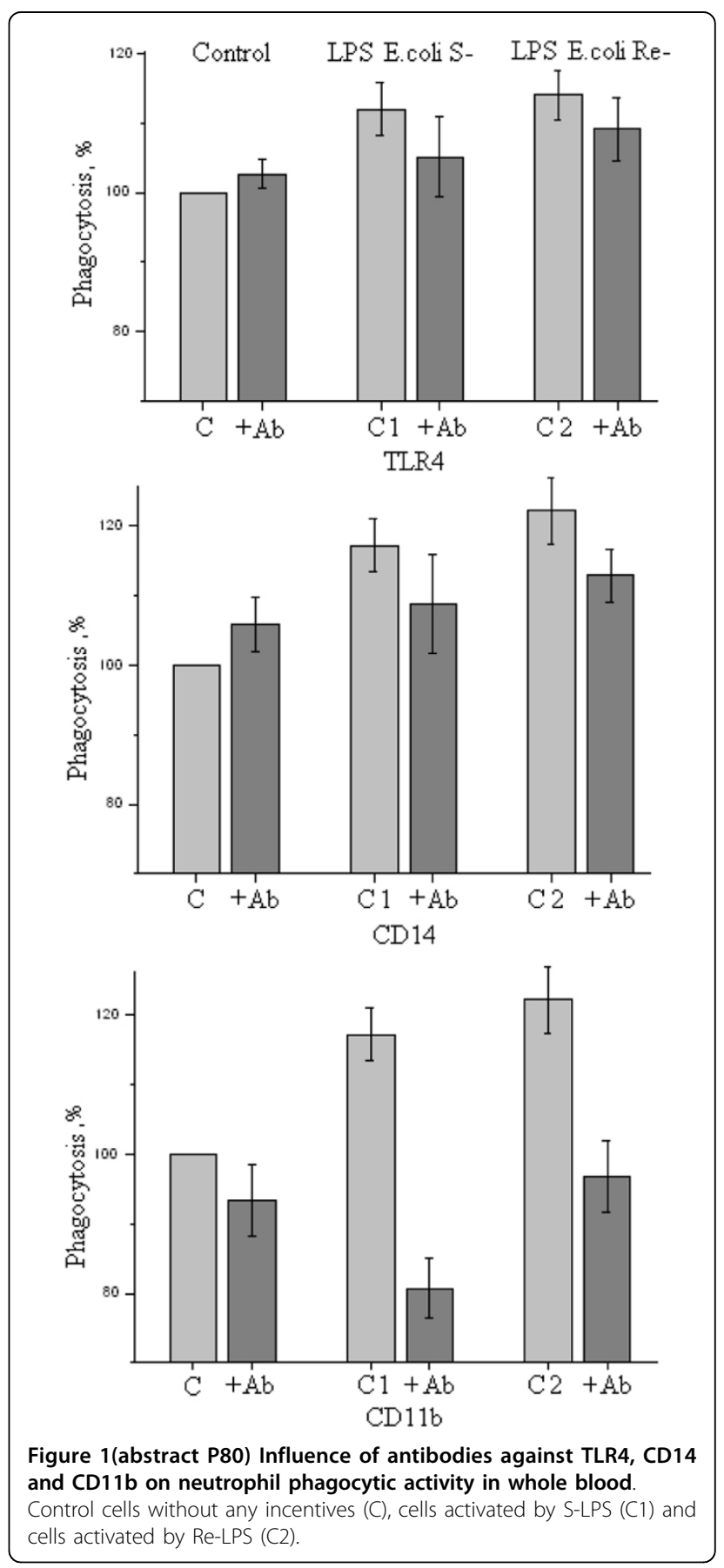


provided by engagement of CD14, TLR4 and CD11b receptors. Thus, we have studied the functional role of these receptors in phagocytosis of fluorescein-labeled Escherichia coli by human neutrophils in whole blood activated by S-form or Re-form endotoxins from $E$. coli.

Methods: S-LPS from E. coli 055:B5 (Sigma) and Re-LPS from E. coli JM103 were obtained in our laboratory according to standard procedure. Fluorescein-labeled bioparticles E. coli K12 (Molecular Probes), mouse antihuman TLR4 mAbs HTA125 (IgG2a isotype; Serotec), anti-human CD14 clone UCHM-1 mAbs (IgG2a isotype; Sigma), and anti-human CD11b mAbs clone 44 (IgG1 isotype; Sigma). LPS were dissolved in pyrogen-free water to make $10 \mu \mathrm{g} / \mathrm{ml}$ stock solution, and sonicated vigorously for 20 minutes to increase LPS solubility. Experiments were performed in whole blood from nine donors (male, female, age 20 to 27). Blood was collected in sterile conditions into Monovette heparin-treated tubes $10 \mathrm{U} / \mathrm{ml}$ (Sarsted, Germany). Each tube contained $90 \mu \mathrm{l}$ whole blood and $50 \mu \mathrm{l}$ suspension of FITC-labeled bacteria $\left(2 \times 10^{7}\right.$ cells $/ \mathrm{ml}$; 1:10 leukocytes: bacteria ratio) was gently mixed and incubated for 30 minutes at $37^{\circ} \mathrm{C}$. Then erythrocytes were lysed by hypotonic buffer for 5 minutes at room temperature. Samples were centrifuged $\left(5\right.$ minutes, $\left.200 \times g, 21^{\circ} \mathrm{C}\right)$ and washed twice by cooled PBS containing $0.02 \%$ EDTA. The pellet was resuspended in $400 \mu \mathrm{l}$ PBS-EDTA solution. To assess the influence of cell activation on phagocytosis the $100 \mathrm{ng} / \mathrm{ml}$ of S-LPS or Re-LPS were added before FITC-labeled bacteria and incubated during 30 minutes at $37^{\circ} \mathrm{C}$. To determine the role of CD14, TLR4 and CD11b receptors in phagocytic activity, corresponding mAbs ( $1 \mu \mathrm{g}, 30$ minutes) were added to whole blood before cell activation by LPS and induction of phagocytosis. The phagocytic activity was monitored using the EPICS XL-MCL flow cytometer (Beckman Coulter). Results given as median fluorescence intensity. Phagocytic activity of cells without LPS activation or mAb treatment was established as $100 \%$. Experiments were done two times using duplicate determinations in each experiment. The mean \pm standard error was calculated using standard calculations available in the OriginPro spreadsheet. Statistically significant differences were determined by Student's $t$ test.

Results: Phagocytic activity of leukocytes was increased by pre-exposure of whole blood to S-LPS or Re-LPS (Figure 1). Pretreatment of whole blood by anti-CD14 or anti-TLR4 mAbs decreased to some extent the phagocytic activity of leukocytes activated by endotoxins independently of their glycoform (Figure 1). Significant inhibition of phagocytosis using antiCD11b mAbs was achieved (Figure 1). Abs against CD11b more pronounced decreased S-LPS activated phagocytosis by $30 \%$ but were less effective in the case of Re-LPS (by 15\%). It is known that CD11b receptors play an important role during phagocytosis of opsonized bacteria.

Conclusion: LPS activate phagocytosis of Gram-negative bacteria independently of their glycoforms (Figure 1). The effect of HTA125 mAbs may be explained by their interaction with TLR4 leading to inhibition of LPS-induced signaling into the nucleus and blocking synthesis and surface expression additional receptors such as class A macrophage scavenger receptor (SR-A) [6] and CD11b [7] involved in phagocytosis. Activation of integrin-dependent signaling pathways can also be blocked by these HTA125 mAbs [8]. Anti-CD $11 \mathrm{~b}$ decreased most pronounced LPSactivated phagocytosis. Taking these results into consideration one can speculate that the Fc-chain of anti-CD11b $\left(\operatorname{lgG}_{1}\right)$ mAbs through interaction with CD32A may mediate downregulation of TLR4 responses. References

1. Fitzgerald K, Rowe D, Golenbock D: Endotoxin recognition and signal transduction by the TLR4/MD-2 complex. Microbes Infect 2004, 6:1361-1367.

2. Qureshi S, Medzhitov R: Toll-like receptors and their role in experimental models of microbial infection. Genes Immun 2003, 4:87-94

3. Zhou X, Gao X, Fan J, Liu Q, Anwar K, Frey R, Malik A: LPS activation of Toll-like receptor 4 signals CD11b/CD18 expression in neutrophils. Am J Physiol Lung Cell Mol Physiol 2005, 288:655-662.

4. Peiser L, DeWinther M, Makepeace K, Hollinshead M, Coull P, Plested J, Kodama T, Moxon E, Gordon S: The class A macrophage scavenger receptor is a major pattern recognition receptor for Neisseria meningitidis which is independent of lipopolysaccharide and not required for secretory responses. Infect Immun 2002, 70:5346-5354.

5. Underhill D, Gantner B: Integration of Toll-like receptor and phagocytic signaling for tailored immunity. Microbes Infect 2004, 6:1368-1373.

6. Fitzgerald M, Moore K, Freeman M, Reed G: Lipopolysaccharide induces scavenger receptor $\mathrm{A}$ expression in mouse macrophages: a divergent response relative to human THP-1 monocyte/macrophages. J Immunol 2000, 164:2692-2700.

7. Brekke $\mathrm{O}$, Christiansen D, Fure H, Fung M, Mollnes T: The role of complement C3 opsonization, C5a receptor, and CD14 in E. coli-induced up-regulation of granulocyte and monocyte CD11b/CD18 (CR3), phagocytosis, and oxidative burst in human whole blood. I Leukoc Biol 2007, 81:1404-1413.

8. Han C, Jin J, Xu S, Liu H, Li N, Cao X: Integrin CD11b negatively regulates TLR-triggered inflammatory responses by activating Syk and promoting degradation of MyD88 and TRIF via Cbl-b. Nat Immunol 2010, 11:734-742.

P81

CD24-mediated neutrophil death in inflammation: ex vivo study suggesting a potential role in sepsis

M Parlato ${ }^{1 *}$, F Souza-Fonseca-Guimaraes ${ }^{1}$, F Philippart ${ }^{2}$, B Misset ${ }^{2}$,

M Adib-Conquy ${ }^{1}$, JM Cavaillon $^{1}$

'Institut Pasteur, Paris, France; ${ }^{2}$ Saint Joseph Hospital, Paris, France Critical Care 2012, 16(Suppl 3):P81

Background: Delayed neutrophil apoptosis is often detected in inflammatory pathologies, including sepsis [1]. CD24 is a small heavily glycosylated cell-surface protein, linked to the membrane by a glycosylphosphatidylinositol (GPI) anchor in a wide variety of cells [2]. Cross-ligation of CD24 triggers apoptosis in a human B-cell subset during the early activation stage [3]. Since CD24 is also expressed on neutrophils, we aimed to study its expression in sepsis and its putative role in apoptosis.

Methods: Blood samples were either collected from healthy donors or from sepsis patients at the onset of sepsis and the two following days and surface CD24 expression on neutrophils was analyzed by flow cytometry. Peripheral blood neutrophils were purified from sepsis patients and healthy individuals by positive selection. Whole blood or neutrophils were challenged with lipopoplysaccharide (LPS), TNF, granulocyte-macrophage colony-stimulating factor (GM-CSF), heat-killed Staphylococcus aureus or heat-killed Escherichia coli and CD24 expression assessed by flow cytometry. Neutrophils were cross-linked with anti-human CD24 and assessed for apoptosis after 24 hours of culture. In some experiments, neutrophils were preincubated with caspase inhibitor (z-VAD-fmk) before crosslinking.

Results: Surface expression of CD24 assessed by flow cytometry was significantly altered in neutrophils from sepsis patients compared with healthy controls. Activation of neutrophils with LPS or heat-killed bacteria in whole blood triggers a strong upregulation of CD24 at surface level despite no enhanced expression being observed when the activation was carried out in purified neutrophils. In contrast, TNF and GM-CSG upregulated CD24 in whole blood and in purified neutrophils. CD24 cross-ligation induces caspase-independent apoptosis in human neutrophils. Ex vivo death responses after CD24 ligation in neutrophils from sepsis patients are currently under study.

Conclusion: This is the first report studying the role of CD24 in sepsis patients, linking homeostasis and apoptosis.

References

1. Bratton DL, Henson PM: Neutrophil clearance: when the party is over, clean-up begins. Trends Immunol 2011, 32:350-357.

2. Fang X, Zheng P, Tang J, Liu Y: CD24: from A to Z. Cell Mol Immunol 2010, 7:100-103.

3. Suzuki T, Kiyokawa N, Taguchi T, Sekino T, Katagiri YU, Fujimoto J: CD24 induces apoptosis in human $B$ cells via the glycolipid-enriched membrane domains/rafts-mediated signaling system. J Immunol 2001, 166:5567-5577.

P82

Resistant Escherichia coli strains circulating in a tertiary-care hospital in New Delhi, India

B Uppal", P Aggarwal, R Ghosh, AK Jha, S Krishna Prakash

Maulana Azad Medical College, New Delhi, India

Critical Care 2012, 16(Suppl 3):P82

Background: Escherichia coli is a leading cause of bloodstream infections worldwide and its associated rate of mortality is high. It is found in its primary habitat, the digestive tract, as a commensal but is also involved in 
various intestinal and extraintestinal diseases. It is a known fact that the digestive tract may serve as a portal of entry into the bloodstream. Therefore, the presence of highly resistant $E$. coli strains in the gut presents a threat to the patients with predispositions such as chronic illnesses and poor immune status.

Methods: This study was undertaken with the aim to determine the resistance pattern among the $E$. coli strains isolated from the gut of chronically ill patients across wide clinical settings. The study was conducted over a period of 1 year from 1 January 2011 to 31 December 2011. Stool samples from patients admitted for more than 14 days in wards of all major clinical specialities were collected after proper counselling and informed consent. E. coli were identified on the basis of cultural characteristics and biochemical reactions. Strains isolated from pediatric patients were subjected to serotyping by the slide agglutination test with specific antisera (Denka Seiken Co., Ltd, Tokyo, Japan) to identify the enterovirulent strains. All E. coli strains were subjected to antimicrobial susceptibility testing and ESBL identification by the disk diffusion methods in accordance with the CLSI guidelines.

Results: Two hundred and fifty-four patients were included in the study with the following distribution: 71 from a pediatrics ward, 62 from a general medicine ward, 54 from a general surgery ward, 42 from a gynaecology ward, 16 from an orthopaedics ward and nine from an ENT ward. E. coli was isolated from 112 samples. Out of $34 \mathrm{E}$. coli strains isolated from paediatric patients, 14 were determined to be enterovirulent $E$. coli by serotyping. Antimicrobial susceptibility testing of all $E$. coli strains showed $100 \%$ resistance to nalidixic acid and a high degree of resistance to ampicillin (87.5\%), doxycyline $(83.0 \%)$, cotrimoxazole $(75.9 \%)$, ciprofloxacin $(73.2 \%)$ and third-generation cephalosporins (71.4\%). ESBL production was detected in 71 strains (63.4\%). However, no resistance was found for carbapenems and tigecycline.

Conclusion: A large population of chronically ill patients who were tested was found to be carrying highly resistant $E$. coli in their guts. These usually commensal strains may serve as a source of bloodstream infection especially in cases of immunosuppression. Whether these strains were acquired in the hospital or from the community needs to be studied further.

\section{P83}

\section{5-Lipoxygenase contributes to PPAR $\gamma$ activation in macrophages in} response to apoptotic cells

A von Knethen ${ }^{*}$, L Eifler', L Kuchler', A Heeg ${ }^{1}$, H Heide², I Wittig², T Maier ${ }^{3}$, D Steinhilber ${ }^{3}$, B Brüne

${ }^{1}$ Institute of Biochemistry I Faculty of Medicine, Goethe-University Frankfurt, Germany; ${ }^{2}$ Faculty of Medicine, Goethe-University Frankfurt, Germany;

${ }^{3}$ Institute of Pharmaceutical Chemistry, Goethe-University Frankfurt, Germany Critical Care 2012, 16(Suppl 3):P83

Background: One hallmark contributing to immune suppression during the late phase of sepsis is macrophage polarization to an anti-inflammatory phenotype upon contact with apoptotic cells (AC). Taking the important role of the nuclear receptor PPAR $\gamma$ for this phenotype switch into consideration, it remains elusive how AC activate PPAR in macrophages. Therefore, we were interested to characterize the underlying principle.

Methods: Apoptosis was induced by treatment of Jurkat T cells for 3 hours with $0.5 \mu \mathrm{g} / \mathrm{ml}$ staurosporine. Necrotic cells (NC) were prepared by heating cells for 20 minutes to $65^{\circ} \mathrm{C}$. PPAR $\gamma$ activation was followed by stably transducing RAW264.7 macrophages with a vector encoding the red fluorescent protein mRuby after PPAR $\gamma$ binding to $4 \times$ PPRE sites downstream of the reporter gene sequence. This readout was established by treatment with the PPAR $\gamma$ agonist rosiglitazone $(1 \mu \mathrm{M})$ and AC (5:1). Twenty-four hours after stimulation, mRuby expression was analysed by fluorescence microscopy. Lipid rafts of AC, NC, as well as living cells (LC) were enriched by sucrose gradient centrifugation. Fractions were analysed for lipid raft-associated marker proteins. Lipid rafts were incubated with transduced RAW264.7 macrophages as described above. 5-Lipoxygenase (5-LO) involvement was verified by pharmacological inhibition (MK-866, $1 \mu \mathrm{M})$ and overexpression.

Results: Assuming that the molecule responsible for PPAR $\gamma$ activation in macrophages is localized in the cell membrane of $A C$, most probably associated to lipid rafts, we isolated lipid rafts from AC, NC and LC. Mass spectrometric analysis of lipid rafts of AC showed the expression of 5-LO, whereas lipid rafts of $L C$ did not. Moreover, incubating macrophages with lipid rafts of $A C$ induced mRuby expression. In contrast, lipid rafts of NC and LC did not. To verify the involvement of 5-LO in activating PPAR in macrophages, Jurkat T cells were incubated for 30 minutes with the 5-LO inhibitor MK-866 $(1 \mu \mathrm{M})$ before apoptosis induction. In line with our hypothesis, these $\mathrm{AC}$ did not induce mRuby expression. Finally, although living Jurkat T cells overexpressing 5-LO did not activate PPAR in macrophages, mRuby expression was significantly increased when $A C$ were generated from 5-LO overexpressing compared with wild-type Jurkat cells. Conclusion: Our results suggest that induction of apoptosis activates 5-LO, localizing to lipid rafts, necessary for PPAR $\gamma$ activation in macrophages. Therefore, it will be challenging to determine whether 5-LO activity in AC, generated from other cell types, correlates with PPAR $\gamma$ activation, contributing to an immune-suppressed phenotype in macrophages.

\section{P84}

Natural killer cell status and tolerance in mouse and human bacterial sepsis

F Souza-Fonseca-Guimaraes ${ }^{1 *}$, M Parlato', F Philippart ${ }^{2}$, B Misset ${ }^{2}$,

JM Cavaillon', M Adib-Conquy', G Captain Study Group

${ }^{1}$ Institut Pasteur, Paris, France; ${ }^{2}$ Groupe hospitalier Paris Saint Joseph, Paris, France Critical Care 2012, 16(Suppl 3):P84

Background: As sensors of infection, innate immune cells are able to recognize pathogen-associated molecular patterns by receptors such as Tolllike receptors (TLRs). Natural killer (NK) cells contribute to inflammatory processes by producing proinflammatory cytokines such as IFN $\gamma$ and GMCSF [1]. Our aim was to characterize the immune status of NK cells in a murine model of sepsis and in patients with systemic inflammatory response syndrome (SIRS) and sepsis.

Methods: Cecal puncture (CP) was employed as a murine model of polymicrobial sepsis. TLR expression in murine and human NK cells was studied by flow cytometry. Ex vivo IFN $\gamma$ production was analyzed either by ELISA or by flow cytometry.

Results: In mice, the expression of TLR2 and TLR4 in spleen NK cells is mainly intracellular, similarly to TLR9. In vitro cell responsiveness of purified NK cells to TLR2, TLR4 or TLR9 agonists, in synergy with accessory cytokines (IL-2, IL-15 and IL-18), allowed a significant production of IFN $\gamma$ and GM-CSF. In contrast, NK cells, purified from spleen of mice with sepsis, showed a dramatic reduction of their capacity to produce cytokines in response to TLR agonists. Depletion of regulatory T cells (Tregs) before CP led to a complete reversion of NK cell tolerance to TLR agonists. IL-10 and TGF- $\beta 1$ are two main inhibitory cytokines produced by Tregs. We showed in vivo, using IL-10 knockout mice and by inhibiting TGF- $\beta$ R signaling, that the tolerization mechanism of NK cells was mostly mediated by TGF- $\beta$ [2]. In humans, the expression of TLR2, TLR4 and TLR9 in peripheral blood NK cells (both CD3 $\mathrm{CD} 56^{\text {high }}$ and $\mathrm{CD} 3^{-} \mathrm{CD} 56^{\mathrm{dim}}$ subsets) was mainly intracellular. The ex vivo responsiveness of the blood NK cells to their agonists in synergy with accessory cytokines (IL-15 and IL-18), allowed a significant secretion of IFN $\gamma$. Similar to the murine model of sepsis, in SIRS and sepsis patients the secretion of IFN $\gamma$ by NK cells was significantly decreased.

Conclusion: NK cells express TLR2 and TLR4 intracellularly, as already reported for other cell types (epithelial, endothelial, and dendritic cells). Furthermore, NK cells undergo tolerance to TLR agonists during SIRS or sepsis, as already described for monocytes in these clinical settings. References

1. Souza-Fonseca-Guimaraes F, et al: Natural killer (NK) cells in antibacterial innate immunity: angels or devils? Mol Med 2012, 18:270-285.

2. Souza-Fonseca-Guimaraes F, et al: NK cell tolerance to Toll-like receptor agonists mediated by regulatory $\mathrm{T}$ cells after polymicrobial sepsis. J Immunol 2012, 188:5850-5858.

P85

Raman spectroscopic investigation of the interaction of Enterococcus faecalis and vancomycin: towards a culture-independent antibiotic susceptibility test

U Neugebauer $^{1 *}$, C Assmann ${ }^{1}$, U Schröder ${ }^{1}$, A Ramoji $^{1}$, U Glaser ${ }^{1}$, C Beleites $^{2}$, W Pfister $^{3}$, J Popp ${ }^{4}$, M Bauer $^{1}$

${ }^{1}$ Center for Sepsis Control and Care, Jena University Hospital, Jena, Germany; ${ }^{2}$ Institute of Photonic Technology, Jena, Germany; ${ }^{3}$ Institute of Medical Microbiology, Jena University Hospital, Jena, Germany; ${ }^{4}$ Institute of Photonic Technology, Institute of Physical Chemistry and Abbe Center of Photonics, Jena, Germany

Critical Care 2012, 16(Suppl 3):P85 

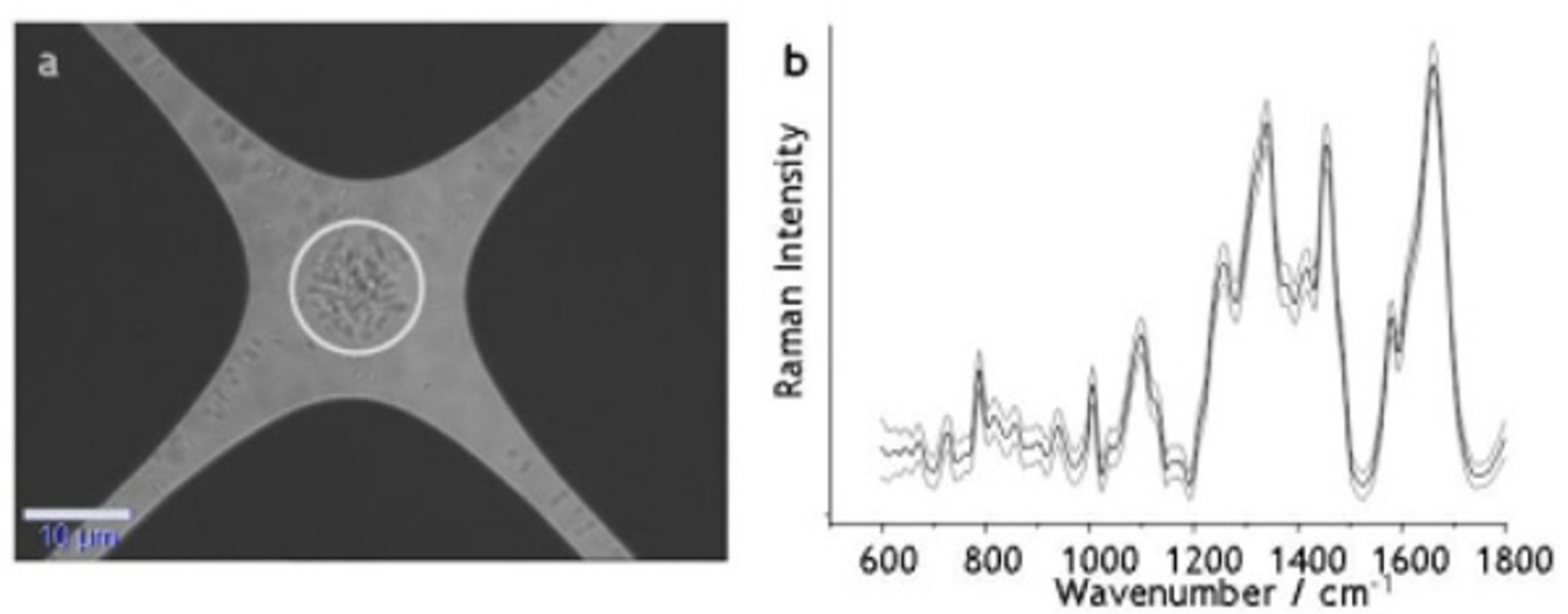

Figure 1(abstract P85) (a) E. faecalis trapped between the electrodes on a quadrupole dielectrophoretic chip. (b) Raman mean spectra with standard deviation taken from 1,000 spectra of the trapped E. faecalis.

Background: Enterococcus faecalis gained importance in the last years for causing tough and troublesome nosocomial infections especially in the urinary tract, sometimes even leading to sepsis. Causes of concern are increasing resistances of enterococci. Therefore, it is of utmost interest to identify resistance patterns of bacteria within a very short timeframe to select the right therapy and efficiently prevent spreading. Within our junior research group we want to develop an on-chip device to probe antibiotic susceptibility patterns of sepsis pathogens based on optical spectroscopy without the need for time-consuming bacterial cultivation. In this contribution, we present our first results with a focus on E. faecalis. Methods: E. faecalis was grown in liquid culture and characterized in the presence and absence of vancomycin by means of Raman spectroscopy. Multivariate statistical analysis was applied to extract the spectral differences due to antibiotic treatment. To enable the spectroscopic analysis directly with patient samples (such as urine) Raman spectroscopy was combined with dielectrophoresis. Bacteria from suspensions were captured and kept at well-defined positions in a nonuniform electric field for the time of Raman measurement. Currently, bacteria-spiked model urine is used. However, the investigations shall be extended to urine from patients.

Results: A clear distinction between Raman spectra of $E$. faecalis with and without antibiotic treatment is possible even 30 minutes after incubation with vancomycin. A quadrupole electrode design is presented that allows the efficient capturing of E. faecalis and Escherichia coli, by means of negative dielectrophoresis [1] (Figure 1a). From the captured bacteria in solution on the dielectrophoretic chip, high-quality Raman spectra have been recorded within 1 second (Figure $1 \mathrm{~b}$ ). These spectra allowed a reliable differentiation of the two commonly encountered bacterial species in urinary tract infections: E. faecalis and E. coli. First steps have been undertaken to implement such dielectrophoretic capturing structures into a microfluidic device for simplified sample handling.

Conclusion: Raman spectroscopy in combination with statistical analysis holds the potential for a fast evaluation of bacterial antibiotic susceptibility without the need of time-consuming cultivation.

Reference

1. Schröder U, Glaser U, Leiterer C, Csáki A, Fritzsche W, Bauer M, Popp J, Neugebauer U: Micromanipulation of sepsis relevant bacteria with dielectrophoresis. Infection 2011, 39(Suppl 2):S104.

P86

Transthoracic echocardiographic assessment of IVC diameter variability to determine fluid responsiveness in children with septic shock: a pilot study

K Sasidaran*, M Jaishree, S Singhi, R Manoj

PGIMER, Chandigarh, India

Critical Care 2012, 16(Suppl 3):P86
Background: Hemodynamic monitoring plays a key role in the early recognition, optimization of interventions and monitoring of therapeutic response in children with septic shock. Assessing the need for fluids followed by rapid and timely fluid resuscitation is crucial for improved outcomes. It is in this context that fluid responsiveness, defined as an increase in cardiac output in response to a fluid challenge, assumes importance. We have done this prospective clinical study to evaluate the degree of IVC diameter variability in predicting fluid responsiveness (increment in stroke index $\geq 15 \%$ ) in children with septic shock post $20 \mathrm{ml} / \mathrm{kg}$ of crystalloid ( $0.9 \%$ saline) resuscitation.

Methods: A total of 166 episodes of preload responsiveness check were echocardiographically evaluated in 41 children with septic shock. In each episode, IVC diameter variability ((maximum - minimum IVC diameter)/ maximum IVC diameter), stroke index and ejection fraction were assessed at two points (before preload T0 and after preload T1). Adequate sedation was ensured before each echocardiographic assessment. Infants $<1$ month of completed age, any clinical evidence of increased intraabdominal pressure, children with previously diagnosed heart disease, requiring high-frequency ventilation, and for whom family did not give consent were excluded from the study.

Results: Of the 166 episodes, 120 (72\%) were fluid responsive and 46 $(28 \%)$ were nonresponsive. One hundred and twenty-five episodes occurred on patients who were on positive pressure ventilation, whilst 41 occurred during spontaneous breathing. The decrease in the heart rate with preload was significant in the responsive as compared with the nonresponsive group ( $24 \pm 9$ vs. $4 \pm 7 ; P=0.001$ ) independent of the ventilator support. IVC diameter variability at T0 correlated significantly ( $r=0.39 ; P=0.001$ ) with stroke index increment following preload. The AUC of ROC for IVC diameter variability was 0.75 (0.66 to 0.85$)$. A cutoff value of $14 \%$ variability showed $84.4 \%$ sensitivity and $65.9 \%$ specificity to positively predict fluid responsiveness in ventilated as well in spontaneously breathing children.

Conclusion: IVC diameter variability showed a significant correlation with stroke index increment after preload and can act as a useful bedside tool in predicting preload responsiveness in children with septic shock. Utility of a serial $\triangle I V C$ percentage instead of a single measurement to assess volume changes needs to be explored.

Do we still accept central venous pressure measurements to assess preload responsiveness in children with septic shock? A single-center experience

K Sasidaran*, M Jaishree, S Singhi

PGIMER, Chandigarh, India

Critical Care 2012, 16(Suppl 3):P87 
Background: Rapid and timely fluid resuscitation is key to improved outcomes in septic shock. Hemodynamic monitoring is vital for preload assessment. Central venous pressure (CVP) is one such traditional tool that is currently falling out of favor as adult data have shown it to be less reliable in predicting preload responsiveness. Pediatric data are, however, limited. We conducted this study to assess the predictive utility of CVP in determining the preload responsive status defined as a stroke index increment $(\geq 15 \%)$ after preload ( $20 \mathrm{ml} / \mathrm{kg} 0.9 \%$ saline) administration.

Methods: A total of 166 episodes of preload administrations were included. Hemodynamic variables (heart rate, mean arterial blood pressure, CVP) were measured before (T0) and after (T1) preload administration in all. Both spontaneously breathing and mechanically ventilated children were enrolled. HR decrement and CVP increment as response to preload were calculated using T1 and T0 measurements. The stroke index was measured by transthoracic echocardiography at T0 and T1. Infants $<1$ month of age, improper position of central venous catheter tip, clinical evidence of increased intra-abdominal pressure, previously diagnosed heart disease, need for high-frequency ventilation, and children in whom family had declined consent were excluded from the study.

Results: Of the 166 episodes, 120 (72\%) were fluid responsive and 46 (28\%) were nonresponsive. One hundred and twenty-five episodes occurred in patients on positive pressure ventilation and 41 during spontaneous breathing. The internal jugular vein was catheterized in 34 (142 episodes; $85 \%$ ) and the femoral vein in seven patients (24 episodes; $14.5 \%$ ). There was a significant decrease in heart rate independent of ventilator support in the preload responsive as compared with the nonresponsive group ( $24 \pm 9$ vs. $4 \pm 7 ; P=0.001)$. However, there was no significant difference in CVP between the fluid responsive and nonresponsive episodes ( $6.2 \pm 1.5$ vs. $6.0 \pm 1.4 ; P=0.71$ ), although CVP was significantly higher in mechanical ventilation as compared with episodes on spontaneous breathing (6.5 \pm 1.3 vs. $5.2 \pm 1.6 ; P=0.001)$. Baseline CVP (T0) $(r=0.01 ; P=0.10)$ and CVP increment at T1 $(r=0.18 ; P=0.14)$ showed a poor correlation with stroke index increment.

Conclusion: CVP increment with preload showed a poor correlation with stroke index increment, suggesting that CVP measurements are not useful in predicting a preload responsiveness state in critically ill children Decrement in heart rate was the only conventional hemodynamic variable that signified preload response but that also in retrospect, thus limiting its utility as a predictive tool.

P88

Emergence of carbapenem resistance in Gram-negative nosocomial

bloodstream infections among critically ill children?

A single-center experience

K Sasidaran*, S Singhi, M Jaishree, A Bansal

PGIMER, Chandigarh, India

Critical Care 2012, 16(Suppl 3):P88

Background: Nosocomial bloodstream infections, especially by Gramnegative bacterial species, contribute to substantial morbidity and mortality in critically ill children. In this context, emerging carbapenem resistance further complicates patient management, as these isolates are often resistant to all $\beta$-lactam antibiotics and frequently co-resistant to most other antibiotics, leaving very few treatment options. We conducted this study to identify the microbiologic spectrum of Gram-negative nosocomial bloodstream infections (BSI) in a PICU of a tertiary care hospital and to determine the changing antibiotic sensitivity pattern.

Methods: Data from prospectively maintained records of patients with more than 48 hours of PICU stay and a Gram-negative bloodstream isolate from January 2009 to December 2011 were analyzed. The study period was divided into Group 1 (January 2009 to December 2009), Group 2 (January 2010 to December 2010), and Group 3 (January 2011 to December 2011) to look for any change in antibiotic sensitivity pattern. Results: There were 102 episodes of Gram-negative BSI (Group 1: 51, Group 2: 33, Group 3: 18) in 1,601 patients, corresponding to 8.2 episodes per 1,000 patient-days. Among the Gram-negative isolates, commonest was Acinetobacter species $(n=44 ; 43 \%)$ followed by Klebsiella pneumonia $(n=16 ; 16 \%)$. All three groups were similar with respect to age, sex ratio, device utilization, grade of infection and outcome. When antibiograms were compared between Groups 1 and 3, there was a significant increase in carbapenem resistance $(19 \%$ vs. $55 \% ; P=0.01)$ over time.
Carbapenem-resistant isolates remained resistant to most of the other antibiotics (third-generation cephalosporins (98\%), aminoglycosides (98\%), $\beta$-lactam with $\beta$-lactamase inhibitor (94\%), ciprofloxacin (90\%)). However, sensitivity to colistin $(100 \%)$ was retained in all carbapenem-resistant Gram-negative isolates. Although Gram-negatives were predominant organisms isolated in all three time periods, a significant increase in fungal isolates ( 3 vs. 12; $P=0.0003$ ) was observed over the study period. All fungal isolates were candida species; nonalbicans candida species (13/19) were commoner than Candida albicans.

Conclusion: Gram-negative bacteria are the commonest cause for nosocomial BSI in our PICU. An increasing trend of carbapenem (imipenam, meropenem) resistance was noted over the study period.

P89

Development of a point-of-care-testing system for procalcitonin

$\mathrm{H} \mathrm{Kim}^{* *}$, ES Yoon ${ }^{2}, \mathrm{~S} \mathrm{Ho}^{2}, \mathrm{SW} \mathrm{Oh}{ }^{3}, \mathrm{EY} \mathrm{Choi}{ }^{2}$

${ }^{1}$ Boditech Med Inc., Chuncheon, Korea; ${ }^{2}$ Hallym University, Chuncheon,

Korea; ${ }^{3}$ Chonbuk National University, Jeonju, Korea

Critical Care 2012, 16(Suppl 3):P89

Background: Sepsis is a daily challenge in ICUs. Today various therapeutic strategies are known to improve survival in patients with sepsis. Early assessment is important for determination of the appropriate treatment. PCT has been proposed as a specific infection maker for this purpose.

Methods: A fluorescence dye-incorporated immunochromatographic assay (FICA) was developed for detection of the PCT concentration in serum. Serum mixed with fluorescence-conjugated detector was loaded onto a cartridge and incubated for 12 minutes. The fluorescence intensity of the cartridge was then measured in a laser fluorescence scanner. The analytical performance of the FICA system was evaluated by precision and recovery tests. The comparability of the new method was examined with an automated analyzer.

Results: A reliable correlation between area ratio (AT/AC), reflecting fluorescence intensity of test/control line, and PCT concentration was observed $(r=0.999)$. The CVs of intra-assay and inter-assay precision in a range of 0.26 to $2.09 \mathrm{ng} / \mathrm{ml}$ were $7.8 \%$ and $4.5 \%$, respectively, and analytical recovery of the FICA system fell within $6.4 \%$ at the tested samples. When the FICA method was compared with Roche Elecsys analyzers, there were strong correlations $(r=0.993, n=80)$.

Conclusion: The FICA system with point-of-care-testing appeared to be an easy, fast and suitable method for measurement of the PCT concentration in serum.

P90

Management of sepsis in Indian ICUs: Indian data from the MOSAICS

study

J Divatia

Tata Memorial Hospital, Mumbai, India

Critical Care 2012, 16(Suppl 3):P90

Background: Indian data from the Management of Sepsis in Asia's Intensive Care Units (MOSAICS) study are presented. The primary objective of the MOSAICS study was to assess the compliance of Asian ICUs and hospitals to the Surviving Sepsis Campaign's 6-hour resuscitation bundle and 24-hour management bundle.

Methods: This was prospective cohort study of 162 adult patients with severe sepsis who were admitted to 17 ICUs in India in July 2009. Patients' baseline characteristics, the achievement of targets within the resuscitation and management bundles, and outcome data were recorded. Main outcome measures were compliance to the Surviving Sepsis Campaign's resuscitation and management bundles. Secondary objectives were to evaluate the impact of compliance on mortality.

Results: The mean age of patients was $53.3 \pm 17$ years. The mean APACHE II SCORE was $21.9 \pm 8.4$, and was significantly higher in nonsurvivors (26.3 \pm 8.5 ) than survivors (19.2 \pm 7.3$)$. Hospital mortality was $38.3 \%(62 / 162)$. Achievement rates for the bundle targets were: lactate measurement, $40.1 \%$ (65/162); blood cultures, $52.4 \%$ (85/162); broad-spectrum antibiotics, $62.3 \%$ (101/162); fluids \pm vasopressors, $84.4 \%$ (103/122); central venous pressure, $49.6 \%$ (56/113); central or mixed venous oxygen saturation, $16.8 \%(19 / 113)$; low-dose steroids, $52.4 \%$ (55/105); glucose control, 27.2\% (44/162); 
lung-protective ventilation, $22.1 \%(15 / 68)$. Compliance rates for the entire resuscitation and management bundles were $6.8 \%(11 / 162)$ and $8 \%(13 / 162)$ respectively. There was a trend to improving survival with bundle compliance. Compliance with the resuscitation and management bundles was associated with mortality rates of $18.2 \%$ and $19 \%$, respectively, while mortality rates with noncompliance were $39.7 \%$ and $41.1 \%$. Of the resuscitation bundle elements, achieving targets for fluid resuscitation and vasopressors was associated with survival, but this disappeared when adjusted for APACHE II score. Of the elements in the management bundle, achieving glucose target and low tidal volume ventilation in patients with acute lung injury as well as the APACHE II score were independently associated with survival.

Conclusion: While mortality from severe sepsis is high, compliance to the resuscitation and management bundles is generally poor in ICUs in India.

\section{P91}

Impact of interventions to reduce device-related infections in Indian cancer centre ICUs

S Myatra*, JV Divatia

Tata Memorial Hospital, Mumbai, India

Critical Care 2012, 16(Suppl 3):P91

Background: Nosocomial infections are important sources of morbidity and mortality in ICUs. Surveillance of nosocomial infection is an integral feature of infection control and quality assurance in hospitals.

Methods: Our ICU is a nine-bed ICU of a tertiary cancer centre in Mumbai, India. Over a 1-year period, between March 2009 and February 2010 (period 1), we prospectively determined the rates of ventilatorassociated pneumonia (VAP) and central line associated bloodstream infection (CLABSI) based on CDC definitions. We also carried out daily process surveillance including compliance to hand hygiene, the ventilator bundle and the central line care bundle. We then introduced the following interventions: routine use of heat-moisture exchanger filters and closed suction systems to reduce VAP, and use of antibiotic-coated central venous catheters to reduce CLABSI. We also reinforced general hygiene practices for all ICU personnel and use of checklists. We then remeasured VAP and CLABSI rates and process compliance over the next 1 year, from March 2010 to February 2011 (period 2).

Results: In period 1, VAP comprised $61 \%$ and CLABSI $35 \%$ of all devicerelated nosocomial infections in the ICU. The CLABSI and VAP rates were 33 and 58 per 1,000 device-days, respectively. VAP and CLABSI accounted for $20 \%$ and $7.7 \%$ increase in mortality, respectively. The hand hygiene compliance rate was $68 \%$ for physicians and $82 \%$ for nurses, and compliance with vascular catheter care and ventilator bundles was $65 \%$ and $70 \%$ respectively. After introduction of the interventions, in period 2 , the incidence of VAP was reduced from 58/1,000 ventilator-days to 30.9 / 1,000 ventilator-days; the CLABSI rate fell to $26.7 / 1,000$ device-days. There was no significant difference in compliance with hand hygiene or the central line and ventilator bundles compared with the previous period.

Conclusion: Our rates of VAP and CLABSI were much higher than those reported for developing countries and the US NHSN. This may be partly due to the immunosuppressed nature of cancer patients with neutropenia and prior chemotherapy. Our interventions resulted in a significant reduction in the rate of VAP, and to a lesser extent for CLABSI. Process compliance did not change. It is essential to improve process compliance to further reduce the incidence of nosocomial infections in our ICU.

P92

Early fluid therapy with splanchnic sympathetic blockage prevented microcirculation damage, gut bacterial overgrowth, bacterial translocation and mortality in sepsis

AMA Liberatore*, EC Del-Massa, LR Gondim, ALB Yamamura, IHJ Koh

Federal University of São Paulo, Brazil

Critical Care 2012, 16(Suppl 3):P92

Background: Herein we examined the hypothesis that preserving splanchnic microcirculation with lasting visceral parasympathetic vasodilatation plus a rapid hyperfluid therapy, during the early phase of severe sepsis $\left(\mathrm{DL}_{60}\right)$, could delay and/or reduce the acutely exacerbating inflammatory feature of sepsis and modify its progression to death. In our previous work, this sepsis determined splanchnic tissue hypoxia, gut bacterial overgrowth, and bacterial translocation (BT) and these events were inflammatory amplification causal factors.

Methods: Adult Wistar-EPM rats $(n=86)$ were anesthetized and submitted to: sepsis (S) (2 $\mathrm{ml}$ Escherichia coli $10^{8} \mathrm{CFU} / \mathrm{ml}$ i.v.) and/or thoracic epidural analgesia (E) $(0.2 \mathrm{ml}$ of $0.05 \%$ bupivacaine) and/or hyperfluid therapy $(\mathrm{H})(30 \mathrm{ml} / \mathrm{kg} / 20$ minutes of Ringer lactate) according to the group (G) design: SG; SEG; SHG; SEHG; EG and; NG (naive). Epidura analgesia and fluid therapy were initiated 30 minutes after sepsis. At the 24-hour period, animals were monitored to: microcirculatory hemodynamics (duodenum, jejunum, ileum, liver and kidney) with
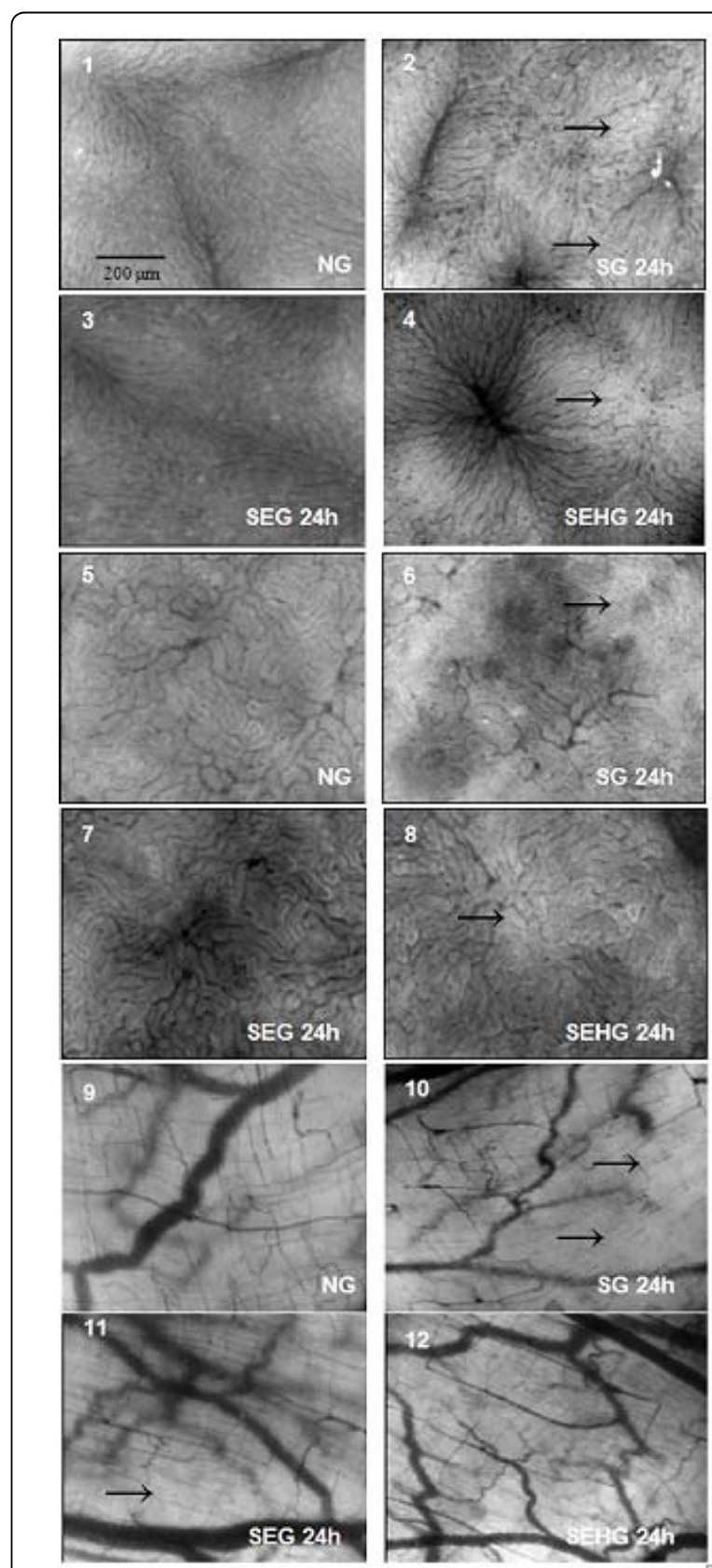

Figure 1(abstract P92) SDF images of organs. Liver (1 to 4) and ileum (9 to 12). Arrows indicate altered microvessels and perivascular tissue areas. 
sidestream darkfield imaging (SDF), gut Gram-negative bacterial count (duodenum, jejunum and ileum) and BT event in mesenteric lymph node (MLN), liver and spleen. Mortality was followed for 30 days.

Results: SDF findings showed that both EG and EHG preserved the microvascular and perivascular tissue architecture following sepsis induction in all organs. However, the SEH combination group showed larger microvessel diameter as compared with SEG, suggesting a better withholding of the intravascular blood volume subsequently improved tissue perfusion (Figure 1). A significant gut bacterial overgrowth was observed only in SG as compared with NG, EG, HG and EHG. The BT index to MLN was proportional to bacterial overgrowth, being higher in SG (67\%), lower in SHG (33\%) and SEG (17\%), and absent in SEHG and EG. The mortality rate was $60 \%$ (SG), 30\% (SEG) and $10 \%(\mathrm{HG})$. All animals with sepsis survived with $\mathrm{E}+\mathrm{H}$ combination therapy.

Conclusion: The splanchnic sympathetic blockage by epidural analgesia attenuated abdominal organs' microcirculatory dysfunction by augmentation of the parasympathetic vasodilatation effect, which might have preserved gut microcirculation, minimizing bacterial overgrowth, BT and subsequent gut immune activation. In addition, vigorous fluid therapy in the early phase of sepsis might have modulated the host systemic inflammatory response by ensuing better tissue perfusion. The combined therapy, by acting differently via an inflammation trigger, might have promoted a synergistic effect. Studies are in progress to verify the combined therapy potentials in the later phase of sepsis.

Acknowledgements: Supported by FAPESP.

\section{P93}

Abdominal organs' microcirculation dysfunction sequence in severe sepsis by SDF microscopy and histology

J Almeida-Filho ${ }^{1 *}$, AMA Liberatore ${ }^{2}$, RC Tedesco ${ }^{2}$, EC Del-Massa², IHJ Koh ${ }^{2}$

${ }^{1}$ Federal University Foundation of Vale do São Francisco, São Paulo, Brazil;

${ }^{2}$ Federal University of São Paulo, Brazil

Critical Care 2012, 16(Suppl 3):P93

Background: The microcirculation dysfunction sequence in sepsis has not been well acknowledged to support therapy and prognosis. Herein, the abdominal organs' microcirculation and their perivascular tissue derangements captured by SDF microscopy were correlated with the whole organ histological findings in severe sepsis.

Methods: Adult Wistar-EPM rats $(n=54)$ were distributed into: sepsis group ( $n=36$ ), animals submitted to $10^{9} \mathrm{CFU} / \mathrm{ml}$ Escherichia coli inoculation through the jugular vein; and sham group $(n=18)$, animals with physiologic saline $0.9 \%$. At 0, 2, 6 and 24 hours, liver, kidney and ileum microvascular and perivascular images were monitored by SDF in vivo and also by histology.

Results: The liver and kidney surface SDF images showed that microcirculation and perivascular tissue alterations are a concomitant event, which were a focal event initially that turned progressively generalized in proportion to sepsis worsening. Vascular patterns were from complete absence to hyperflow and narrowed to dilated venules, showing that altered and nonaltered microvessels occur simultaneously in sepsis. The expansion of perivascular tissue fuzziness with narrowed or vanished microvessels in sepsis suggested local dysfunction in progression. Confronting these areas with histology, the enlarged perivascular areas were composed mostly of cytoplasm edema at the early sepsis phase and of varying stages of the cell necrosis process at the further periods. These processes were very similar in both the liver and kidney and occurred throughout the organ, showing that the surface findings can be extensive to deeper areas (Figure 1). In contrast, the small bowel SDF images showed that gut surface microcirculation was composed primarily of high-flow capillaries and only the vascular density was focally reduced in sepsis; besides, the perivascular tissues could not be identified, showing that the vascular and tissue architectural pattern in the gut differs substantially from solid organs, suggesting a different pathophysiology response of the intestinal microcirculation in sepsis. The gut histology showed generalized cell edema and varying necrosis phases in muscle layers of the intestine in sepsis, and such events could not be suspected by SDF.

Conclusion: Organ dysfunction in sepsis is better detectable in solid organs by SDF imaging as compared with gut muscular compartment.
These results demonstrated the importance of the solid organ SDF monitoring in experimental sepsis treatment studies.

Acknowledgements: Supported by FAPESP.

P94

Role of the clarithromycin immune modulator activity on abdominal

microhemodynamics and mortality in severe sepsis

FRE Jesus*, CD Snak-Souza, FM Dulcini, EC Del-Massa, LR Gondim,

AMA Liberatore, IHJ Koh

Federal University of São Paulo, Brazil

Critical Care 2012, 16(Suppl 3):P94

Background: The management of the uncontrolled systemic inflammatory response in the early phase of severe sepsis is still a challenge. Herein we aimed to minimize the acute inflammation phase of sepsis by clarithromycin associated with intravascular volume restoration with an aggressive fluid therapy.

Methods: Wistar rats were distributed into five groups: $S$, animals submitted to sepsis ( $\left(\mathrm{DL}_{80} ; 3 \mathrm{ml}\right.$ Escherichia coli $10^{9} \mathrm{CFU} / \mathrm{ml}$, i.v., $n=6$ ); $\mathrm{SCH}$, animals induced to sepsis after clarithromycin $(14 \mathrm{mg} / \mathrm{kg}$, i.v., given 24 and 0 hours before sepsis) and hyperhydration (Ringer lactate $40 \mathrm{ml} / \mathrm{kg}$, i.v., in 20 minutes after the sepsis induction) $(n=6) ; \mathrm{SC}$, animals induced to sepsis and treated with clarithromycin $(n=6) ; \mathrm{SH}$, animals induced to sepsis and treated with hyperhydration $(n=6)$; and Sham, animals induced to sham sepsis $(n=4)$. All invasive procedures were undergone after general anesthesia. The liver, kidney and ileum microcirculation were monitored by Laser Doppler and sidestream darkfield imaging at 2-hour and 26 -hour periods. The mortality index was observed for 30 days.

Results: All animals of the SCH and Sham groups survived while the S, SC and SH groups showed $20 \%$ survival. Hyperhydration or clarithromycin in sepsis showed a partial and transient improvement of the microcirculation of the abdominal organs, although the association of hyperhydration with clarithromycin (SCH group) showed better effects. In addition, the beneficial microhemodynamic effect under combined therapy was better evidenced in the liver and intestine as compared with the kidney.

Conclusion: The association of clarithromycin and hyperhydration showed a beneficial effect in severe sepsis, possibly by modulating inflammatory response and microcirculation damage, respectively, resulting in subsequent survival; nevertheless, their individual effects were not efficient to inhibit severe sepsis mortality and microcirculation dysfunction.

Acknowledgements: Supported by FAPESP.

P95

Preliminary results for the use of proteinase $K$ to achieve release of

LPS from the Alteco LPS Adsorber ${ }^{\circledR}$ after perfusion with LPS containing blood

E Hansen $^{1 *}$, L Pierre $^{2}$, S Blomqvist ${ }^{1,2}$

${ }^{1}$ Alteco Medical AB, Lund, Sweden; ${ }^{2}$ Faculty of Medicine, Lund University, Sweden

Critical Care 2012, 16(Suppl 3):P95

Background: The effect of Alteco LPS Adsorber ${ }^{\oplus}$ to remove LPS from the circulation is based on the incorporation of a synthetic peptide that binds to the lipid A moiety of LPS, the binding capacity in one adsorber exceeding $7.5 \mu \mathrm{g}$ LPS. Positive clinical effects of the adsorber when used in patients with Gram-negative sepsis have been reported [1]. Measurement of LPS in human blood, however, is hampered by difficulties such as contamination and interference. The aim of this pilot study was to evaluate a method to release LPS from the LPS adsorber after perfusion with LPS containing blood.

Methods: Two adsorbers (A1, A2) containing the active peptide and one dummy adsorber (D) with no peptide were used. Whole blood $(500 \mathrm{ml})$ from healthy pigs was collected in a bag containing heparin, $37 \mu \mathrm{g}$ LPS was added. A roller pump was used to recirculate the blood through the adsorber. The pump flow was set at $150 \mathrm{ml} /$ minute and the duration of the perfusion was 2 hours. After the end of perfusion the adsorbers were rinsed with $500 \mathrm{ml} \mathrm{Krebs} \mathrm{solution.} \mathrm{A} \mathrm{solution} \mathrm{of} 20 \mathrm{mg}$ proteinase $\mathrm{K}$ in $50 \mathrm{ml}$ Tris buffer with $\mathrm{pH} 7.4$ was prepared and $2.5 \mathrm{ml} \mathrm{CaCl}$ was added. The adsorbers were kept at $37^{\circ} \mathrm{C}$ and the proteinase $\mathrm{K}$ solution [2] was perfused through 


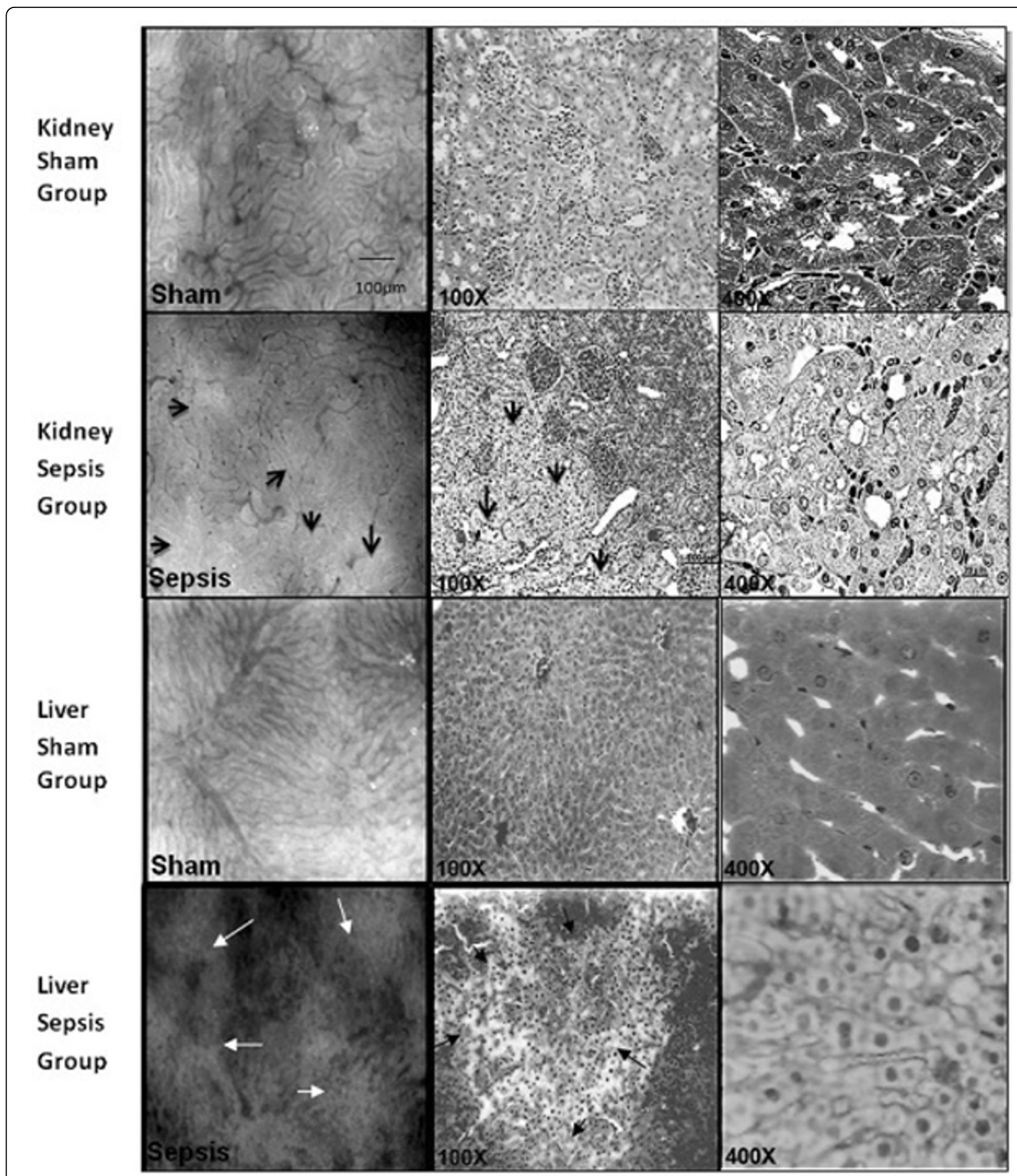

Figure 1(abstract P93) Organ SDF and histological findings in sham and sepsis groups at the 6-hour period. Arrows indicate vascular and perivascular tissue derangements.

the adsorbers at $5 \mathrm{ml} /$ minute for 6 hours. Samples for LPS analysis (chromogenic LAL) [3] were drawn before the perfusion and then after 30, 240 and 360 minutes. The enzyme activity was checked using a synthetic substrate [4].

Results: The concentrations of LPS before and during perfusion with proteinase $\mathrm{K}$ are shown in Table 1.
Conclusion: The LPS values found before the start of the perfusion indicate contamination of the solution. The increase in LPS seen in all adsorbers after 30 minutes is probably due to traces of blood components. The later increase in LPS after treatment with proteinase $\mathrm{K}$ in the active adsorbers indicates that the adsorber is effective in capturing LPS from whole blood and that proteinase $\mathrm{K}$ is able to dislodge LPS bound to the adsorber. 
Table 1(abstract P95)

\begin{tabular}{lllll}
\hline LPS & Before & $\mathbf{3 0}$ minutes & $\mathbf{2 4 0}$ minutes & $\mathbf{3 6 0}$ minutes \\
\hline A1 $(\mathrm{EU} / \mathrm{ml})$ & 3.65 & 5.29 & 27.04 & 50.68 \\
A2 $(\mathrm{EU} / \mathrm{ml})$ & 2.74 & 4.65 & 10.45 & 18.04 \\
D (EU/ml) & 3.94 & 4.16 & 6.78 & 5.82 \\
Enzyme activity (\%), A1, A2, D & $100,100,100$ & $104,110,110$ & $96,102,104$ & $92,95,102$ \\
\hline
\end{tabular}

\section{References}

1. Ala-Kokko Tl, Laurila J, Koskenkari J: A new endotoxin adsorber in septic shock: observational case series. Blood Purif 2011, 32:303-309.

2. Petsch D, Deckwer WD, Anspach FB: Proteinase K digestion of proteins improves detection of bacterial endotoxin by the Limulus amebocyte lysate assay: application for endotoxin removal from cationic proteins. Anal Biochem 1998, 259:42-47.

3. European Pharmacopeia EDQM, 6 2008, 1

4. Bajorath J, Hinrichs W, Saenger W: The enzymatic activity of proteinase K is controlled by Calcium. Eur J Biochem 1988, 176:441-447.

P96

PSP/reg and NT-proCNP to predict the occurrence of ICU-acquired sepsis in severe trauma patients: results of a pilot study

M Dupin ${ }^{1 *}$, M Chanteperdrix', I Chaillol', A Pachot ${ }^{1}$, F Venet ${ }^{2}$, G Monneret ${ }^{2}$,

B Allaouchiche ${ }^{2}$, A Gouel-Chéron ${ }^{2}$

${ }^{1}$ bioMérieux, Lyon, France; ${ }^{2}$ Hospices Civils de Lyon, France

Critical Care 2012, 16(Suppl 3):P96

Background: Major trauma is characterized by a proinflammatory response, followed by an immunosuppression, increasing the risk for ICU acquired infection. Several prognostic markers of sepsis in ICU patients have been identified and need to be assessed. Recently, Pancreatic Stone Protein/regenerating protein (PSP/reg) was shown to be increased during post-traumatic sepsis [1]. The N-terminal fragment of the C-type natriuretic peptide precursor (NT-proCNP) was assessed as a predictor of sepsis in multiple traumatized patients without brain injury [2]. The main objective of this study was to determine whether measurements of those biomarkers could help in the prediction of sepsis in severe trauma patients.

Methods: This retrospective observational study was carried over 24 months in a trauma ICU at a university hospital. Trauma patients under mechanical ventilation with an Injury Severity Score (ISS) $>25$ and age $>18$ were included. Patients dying in the first 48 hours after trauma, having pulmonary inhalation/gut perforation during trauma or after the onset of infection, were excluded. We used already described ELISA protocols for the detection of PSP/reg [1] and NT-proCNP (Biomedica, BI-20872). Both biomarkers have been measured every 2 days from day 1 to day 4 after trauma. After descriptive analysis of clinical data (using medians with interquartile ranges), we evaluated the standard fold-change (SFC) and the area under the curve (AUC) between patients being infected (sepsis group) and patients not being (nonsepsis group).

Results: We analyzed 61 trauma patients: age $37(25 ; 50)$, ISS $38(33 ; 45)$. Among them, 24 developed sepsis in the first week after trauma (pneumonia; median delay 4 days). There were no differences between sepsis and nonsepsis groups at admission regarding demographic data. Neither PSP/reg nor NT-proCNP showed significant differences between sepsis and nonsepsis groups, whatever time point was considered (respectively days 1 to 2 and days 3 to 4; Table 1). The computed values for SFC and AUC were lower than the minimal detectable values.

Conclusion: In this pilot study, neither PSP/reg nor NT-proCNP can help in the prediction of sepsis in severe trauma patients. Contrary to the results published by Bahrami and colleagues [2], we did not show significant difference between sepsis and nonsepsis groups in patients without brain injury. Our study illustrates the complexity of validating biomarkers for sepsis prediction in independent cohort of patients.

References

1. Keel $\mathrm{M}$, et al: Pancreatic stone protein is highly increased during posttraumatic sepsis and activates neutrophil granulocytes. Crit Care Med 2009, 37:1642-1648.

2. Bahrami S, et al: Circulating NT-proCNP predicts sepsis in multipletraumatized patients without traumatic brain injury. Crit Care Med 2010, 38:161-166.

P97

From positive blood culture to microbiological diagnosis in 4 hours by MALDI-TOF mass spectrometry bacterial identification and rapid antibiogram

S Barnini, V Brucculeri, P Morici, A Lupetti, M Campa

Azienda Ospedaliero - Universitaria Pisana, Pisa, Italy

Critical Care 2012, 16(Suppl 3):P97

Background: Time is precious for patients and clinicians facing septic events. In recent past years, many efforts have been made to develop rapid and reliable tests, especially by molecular methods, which still have several limits in determining antimicrobial susceptibility. Nowadays, MALDI-TOF mass spectrometry allows almost instantaneous bacterial identification, but antibiotic susceptibility results are usually delayed 24 to 48 hours, compared with the timing of microorganism identification.

Methods: In this work, two short procedures, one for Gram-negative and one for Gram-positive bacteria, were developed. The two methods were employed to purify bacteria from positive blood cultures (Becton Dickinson) after microscopic examination, and to prepare bacterial cells for identification. Bacteria were then identified by MALDI-TOF mass spectrometry (Bruker Daltonics) with excellent scores, similar to those obtained from agar plate colonies. After identification, short-term broth cultures were inoculated with an aliquot of the recovered bacteria, with or without (growth controls) antibiotics and incubated in an HB\&L (Alifax $\mathrm{SpA}$ ) instrument, which monitored each vial for bacterial growth during the following 3 hours. Absence or reduction in growth in a vial containing a certain antibiotic was interpreted as sensibility, while resistance was assessed by a bacterial growth comparable with the control vial.

Results: In these pilot experiments $32 \mathrm{Gram}$-negative (10 Klebsiella pneumoniae, six Escherichia coli, four Enterobacter aerogenes, four Pseudomonas aeruginosa, two Klebsiella oxytoca, two Acinetobacter baumannii, one Citrobacter freundii, one Proteus mirabilis, one Serratia

Table 1(abstract P96) PSP/reg and NT-proCNP doses $(\mathrm{ng} / \mathrm{ml})$ in function of patient groups

\begin{tabular}{llll}
\hline & & Sepsis & Nonsepsis \\
\hline PSP/reg & Days 1 to $2(n=38)$ & $(n=16) 30.1(15.9 ; 51.4)$ & $(n=22) 27.8(18.0 ; 41.3)$ \\
& Days 3 to $4(n=36)$ & $(n=7) 57.9(21.7 ; 131.1)$ & $(n=29) 38.5(15.8 ; 71.2)$ \\
NT-proCNP & Days 1 to $2(n=37)$ & $(n=16) 4.5(3.6 ; 5.7)$ & $(n=21) 5(3.8 ; 8.8)$ \\
& Days 3 to $4(n=36)$ & $(n=7) 3.6(3.3 ; 3.9)$ & $(n=29) 4.7(3.3 ; 5.6)$ \\
\hline
\end{tabular}

Data presented as median (Q1; Q3). 
marcescens, one Acinetobacter Iwoffii) and 46 Gram-positive (28 Staphylococcus epidermidis, eight Staphylococcus hominis, three Staphylococcus capitis, two Staphylococcus haemolyticus, two Staphylococcus aureus, one Staphylococcus auricularis, one Enterococcus faecalis, one Enterococcus faecium) bacterial isolates were correctly identified and 274 rapid susceptibility tests were performed. Among these, 259 were compared with results from Vitek 2 (bioMérieux), obtained 48 hours later from agarplated cultures of routine analysis, with an overall agreement of $93 \%(1.5 \%$ very major errors, $4 \%$ major errors, $1 \%$ minor errors; discrepancies resolved by E-test). Antibiotics tested were chosen among cefotaxime, ceftazidime, gentamicin, levofloxacin, meropenem, amikacin and colistin for Gramnegative bacteria and cefoxitin, linezolid, teicoplanin and ampicillin for Gram-positive bacteria. All but cefoxitin gave results in 3 hours.

Conclusion: These results show that rapid and reliable microbiological answers can be given in a few hours, thus allowing clinicians to start a proper and lifesaving antibiotic therapy.

P98

Relationship between plasma NGAL and serum creatinine is influenced by leucocytosis and neutrophilia in the critically ill

NJ Glassford ${ }^{1 *}$, AG Schneider', G Eastwood ${ }^{1}$, L Peck' $^{1}, \mathrm{H}$ Young ${ }^{1}, \mathrm{~S} \mathrm{Xu}^{2}$, R Bellomo ${ }^{3}$

'Austin Hospital, Melbourne, Australia; ${ }^{2}$ Uppsala University, Uppsala, Sweden; ${ }^{3}$ Monash University, Melbourne, Australia

Critical Care 2012, 16(Suppl 3):P98

Background: NGAL appears to be a biomarker of acute kidney injury. It is produced by neutrophils and renal tubular cells. In healthy patients, plasma NGAL levels have been shown to vary with white blood cell concentrations. The predictive ability of NGAL to predict AKI is attenuated by leucocytosis in patients being treated with vancomycin. The relationship between NGAL and white cell indices in the critically ill is poorly understood. The aim is to explore the relationship between different measures of NGAL and white cell count (WCC) and neutrophil count (NC) in critically ill patients at risk of AKI. Methods: Patients with systemic inflammatory response syndrome and oliguria, or a creatinine rise $>25 \mu \mathrm{mol} / \mathrm{l}$, or both, in a tertiary referral university hospital ICU, within 48 hours of admission, were studied. We measured kidney-specific monomeric urinary NGAL (muNGAL), plasma (pNGAL) and urinary NGAL (ucNGAL) by commercial assay, and routine haematological and biochemical parameters. Institutional ethics approval was obtained.

Results: We studied 84 patients (51.2\% male, median age 66.5 (52 to 74 )) with a median APACHE III score of 61 (45 to76). Fifty-one patients had WCC $>10 \times 10^{9} / \mathrm{l}$, 58 had NC $>7.5 \times 10^{9} /$. No useful correlations were demonstrated between white cell indices and any form of NGAL measurement. A stronger correlation existed between creatinine and sNGAL than urinary indices. The strength of correlation between creatinine and all measures of NGAL was greater in those patients with high white cell indices (Table 1); the increase was significant for sNGAL in those with elevated WCC $(r=0.27$ in nonleucoytotic patients, $r=0.65$ in leucocytotic patients, $P<0.05$ ).

Conclusion: The stronger correlation between creatinine and pNGAL suggests systemic factors may be more important than local factors in the pathophysiology of AKI. The increased strength of relationship in those with leukocytosis suggests NGAL may be a general index of illness severity. Multicentre and longitudinal studies are required to further increase our understanding of the natural history of NGAL release and the clinical utility of these tests.

Table 1(abstract P98) Spearman correlation for inclusion creatinine

\begin{tabular}{lllll}
\hline Group & $\boldsymbol{n}$ & pNGAL & ucNGAL & muNGAL \\
\hline Undifferentiated & 84 & 0.51 & 0.40 & 0.40 \\
No leucocytosis $\left(\right.$ WCC $\left.<10 \times 10^{9} / \mathrm{I}\right)$ & 33 & 0.27 & 0.22 & 0.21 \\
Leucocytosis (WCC $\left.>10 \times 10^{9} / \mathrm{I}\right)$ & 51 & 0.65 & 0.47 & 0.47 \\
No neutrophilia $\left(\mathrm{NC}<7.5 \times 10^{9} / \mathrm{I}\right)$ & 26 & 0.40 & 0.33 & 0.32 \\
Neutrophilia $\left(\mathrm{NC}>7.5 \times 10^{9} / \mathrm{I}\right)$ & 58 & 0.55 & 0.42 & 0.41 \\
\hline
\end{tabular}

P99

Corticosteroid resistance in sepsis is influenced by microRNA-124-

induced downregulation of glucocorticoid receptor- $\alpha$

P Möhnle ${ }^{1 *}$, C Ledderose ${ }^{2}$, J Briegel ${ }^{1}$, S Kreth

'Ludwig-Maximilians-Universität München, Klinik für Anaesthesiologie,

München, Germany; ${ }^{2}$ Universitätsklinikum Mannheim, Klinik für

Anästhesiologie und Intensivmedizin, Mannheim, Germany

Critical Care 2012, 16(Suppl 3):P99

Background: Acquired glucocorticoid resistance frequently complicates the therapy of sepsis. It leads to an exaggerated proinflammatory response and has been related to altered expression profiles of glucocorticoid receptor isoforms glucocorticoid receptor- $\alpha$ (mediating anti-inflammatory effects) and glucocorticoid receptor- $\beta$ (acting as a dominant negative inhibitor). We investigated the impact of glucocorticoid receptor isoforms on glucocorticoid effects in human T cells. We hypothesized that changes of the ratio of glucocorticoid receptor isoforms impact glucocorticoid resistance and that glucocorticoid receptor- $\alpha$ expression is controlled by microRNA-mediated gene silencing.

Methods: First, $T$ cells from healthy volunteers (native and CD3/CD28stimulated cells with or without addition of hydrocortisone) were analyzed for the expression of glucocorticoid receptor isoforms by quantitative PCR. Additionally, effects of gene silencing of glucocorticoid receptor- $\beta$ by siRNA transfection were determined. Secondly, microRNA-mediated silencing was evaluated by cloning of a glucocorticoid receptor- $\alpha$-specific 3 '-untranslatedregion reporter construct and subsequent transfection experiments in cell cultures. Effects of miRNA transfection on glucocorticoid receptor- $\alpha$ expression were analyzed in Jurkat $\mathrm{T}$ cells and in T cells from healthy volunteers (quantitative PCR and western blotting). Finally, expression of glucocorticoid receptor- $\alpha$, glucocorticoid receptor- $\beta$, and miR-124 was tested in T cells of sepsis patients $(n=24)$.

Results: Stimulation of T cells induced a significant upregulation of glucocorticoid receptor- $\alpha$ (not glucocorticoid receptor- $\beta$ ), thereby possibly rendering T cells more sensitive to glucocorticoids; this T-cell response was hindered by hydrocortisone. Silencing of glucocorticoid receptor- $\beta$ doubled the inhibitory effects of glucocorticoids on IL-2 production. MicroRNA-124 was proved to specifically downregulate glucocorticoid receptor- $\alpha$. Furthermore, a glucocorticoid-induced threefold upregulation of microRNA-124 was found. T cells of sepsis patients exhibited slightly decreased glucocorticoid receptor- $\alpha$ and slightly increased miR-124 expression levels, whereas glucocorticoid receptor- $\beta$ expression was twofold upregulated $(P<0.01)$ and exhibited a remarkable interindividual variability.

Conclusion: Glucocorticoid treatment induces expression of miR-124, which downregulates glucocorticoid receptor- $\alpha$ thereby limiting antiinflammatory effects of glucocorticoids. Steroid treatment might aggravate glucocorticoid resistance in patients with high glucocorticoid receptor- $\beta$ levels.

Acknowledgements: This work has recently been published in Critical Care Medicine: Ledderose C, Möhnle P, Limbeck E, Schütz S, Weis F, Rink J, Briegel J, Kreth S: Corticosteroid resistance in sepsis is influenced by microRNA-124-induced downregulation of glucocorticoid receptor- $\alpha$. Crit Care Med 2012 [Epub ahead of print]. ${ }^{\circ}$ Society of Critical Care Medicine and Lippincott Williams \& Wilkins, and the presented excerpt for Sepsis 2012 Paris was permitted by Wolters Kluwer Health, 8 August 2012.

\section{P100}

Glucocorticoids control systemic inflammatory response by regulation of energy metabolism and cytokine expression A Kleyman ${ }^{1,2 *}$, S Hübner ${ }^{2}$, M Bauer ${ }^{1}$, JP Tuckermann ${ }^{2,3}$

${ }^{1}$ Center for Sepsis Control and Care, Jena University Hospital, Jena, Germany; ${ }^{2}$ Leibniz Institute for Age Research, Jena, Germany; ${ }^{3}$ University of UIm, Institute for General Zoology and Endocrinology, Ulm, Germany Critical Care 2012, 16(Suppl 3):P100

Background: Endogenous glucocorticoids (GCs) are essential for survival in systemic inflammatory response (SIRS). So far, their effect has been attributed exclusively to anti-inflammatory activities of GCs in leukocytes. GCs act through glucocorticoid receptor (GR), a ubiquitously expressed 
nuclear receptor. GR binds as a homodimer to the glucocorticoid responsive element in the promoter of target genes and regulates their transcription. Another mode of GR action is dimerization independent; monomeric GR interacts with other transcriptional factors such as AP1, $\mathrm{NF}-\kappa \mathrm{B}, \mathrm{IRF} 3$, modulates their activity and in this way regulates the expression of target genes. Our study explored which cells mediate the beneficial effects of GCs and identified GR target genes important for survival in systemic inflammatory response.

Methods: Knock-in mice carrying a dimerization-deficient GR (GRdim mice), leptin-deficient mice (ob/ob mice) and the respective wild-type littermates were subjected to a LPS-induced shock model.

Results: After high-dose LPS (10 mg/kg) wild-type mice decreased oxygen consumption and developed mild temporal hypothermia; however, in 6 to 12 hours they discontinued to decrease energy metabolism and recover during 24 to 48 hours. Recovery of energy metabolism in wild-type mice was accompanied by upregulation of leptin expression in white adipose tissue and an increase of leptin serum level as well as upregulation of expression of the leptin target gene (proopiomelanocortin (POMC)) in the hypothalamus. After LPS injection, mice with a dimerization-deficient GR (GRdim mice) had normal TNF $\alpha$ and elevated IL-1 $\beta$ serum level compared with wild-type littermates. GRdim mice developed a strong repression of oxygen consumption, severe hypothermia and died during 48 hours after LPS administration. Continued repression of energy metabolism in GRdim mice was associated with the inability to increase leptin serum level and a misregulation of leptin signaling in hypothalamus after LPS injection. Leptindeficient ob/ob mice demonstrate phenotype similar to GRdim mice: a strong suppression of energy metabolism, low oxygen consumption, severe hypothermia, and increased lethality in systemic inflammation. Importantly, treatment with IL-1 receptor antagonist (IL-1RA) alone does not rescue GRdim mice; however, simultaneous treatment with IL-1RA and leptin significantly increase energy metabolism and rescue survival of GRdim mice in LPS-induced SIRS.

Conclusion: We show that endogenous GCs fulfill their protective function during systemic inflammation by different mechanisms: repression of cytokine expression and regulation of energy metabolism via upregulation of leptin expression.

\section{P101}

Assessment of clinical deterioration and progressive organ failure in

moderate-severity emergency department sepsis patients

R Arnold", L Glaspey, S Hollenberg, S Trzeciak

Cooper University Hospital, Camden, NJ, USA

Critical Care 2012, 16(Suppl 3):P101

Background: The PRE-SHOCK population, ED sepsis patients with tissue hypoperfusion (lactate of 2.0 to $3.9 \mathrm{mM}$ ), commonly deteriorates after admission and requires transfer to critical care. The objective was to determine the physiologic parameters and disease severity indices in the ED PRE-SHOCK sepsis population that predict clinical deterioration. We hypothesized that neither initial physiologic parameters nor organ function scores will be predictive.

Methods: A retrospective analysis of a prospectively maintained registry of sepsis patients with lactate measurements in an urban, academic medical center. The PRE-SHOCK population was defined as adult ED sepsis patients with either elevated lactate (2.0 to $3.9 \mathrm{mM}$ ) or transient hypotension (any $\mathrm{sBP}<90 \mathrm{mmHg}$ ) receiving intravenous antibiotics and admitted to a medical floor. Consecutive patients meeting PRE-SHOCK criteria were enrolled over a 1-year period. Patients with overt shock in the ED, pregnancy, or acute trauma were excluded. The primary patient-centered outcome was increased organ failure (Sequential Organ Failure Assessment (SOFA) score increase $>1$ point, mechanical ventilation or vasopressor utilization) within 72 hours of admission or in-hospital mortality.

Results: We identified 248 PRE-SHOCK patients from 2,649 screened. The primary outcome was met in $54 \%$ of the cohort and $44 \%$ were transferred to the ICU from a medical floor. Patients meeting the outcome of increased organ failure had a greater shock index $(1.02$ vs. $0.93, P=0.042)$ and HR (115 vs. $105, P<0.001)$ with no difference in initial lactate, age, MAP or exposure to hypotension (sBP $<100 \mathrm{mmHg}$ ). There was no difference in the Predisposition, Infection, Response, and Organ dysfunction (PIRO) score between groups (6.4 vs. 5.7, $P=0.052$ ). Outcome patients had similar initial levels of organ dysfunction but had higher SOFA scores at 24,48 , and 72 hours, a higher ICU transfer rate ( 60 vs. $24 \%, P<$ 0.001 ) and increased ICU and hospital lengths of stay.

Conclusion: The PRE-SHOCK sepsis population has a high incidence of clinical deterioration, progressive organ failure, and ICU transfer. Physiologic data in the ED were unable to differentiate the PRE-SHOCK sepsis patients who developed increased organ failure. This study supports the need for an objective organ failure assessment in the emergency department to supplement clinical decision-making.

\section{P102}

Increased endotoxin activity is associated with clinical deterioration in moderate-severity emergency department sepsis patients: a pilot study R Arnold*, C Schorr, S Trzeciak, RP Dellinger

Cooper University Hospital, Camden, NJ, USA

Critical Care 2012, 16(Suppl 3):P102

Background: Increasing levels of endotoxin activity are associated with increasing mortality in ICU patients. The removal of endotoxin through polymyxin $B$ hemoperfusion has been shown to decrease organ dysfunction and reduce mortality in septic shock patients. Our objective was to determine the ability of endotoxin measurement and its change over 24 hours in normotensive sepsis patients to predict clinical deterioration.

Methods: A prospective observational study in a single-center urban, academic medical center involving adult ED patients with suspected infection admitted to a medical floor and receiving i.v. antibiotics with either elevated lactate (2.0 to $3.9 \mathrm{mM}$ ) or transient hypotension (sBP $<90$ $\mathrm{mmHg}$ ). Patients with overt shock (mechanical ventilation or vasopressor requirement), pregnancy, or acute trauma were excluded. The Endotoxin Activity Assay was measured at enrollment and again at 24 hours. Subjects were followed for the development of increased organ failure (Sequential Organ Failure Assessment (SOFA) score increase $>1$ point, mechanical ventilation or vasopressor utilization) within 72 hours of admission or inhospital mortality.

Results: We enrolled 57 patients over a 12-month period. The primary outcome was met in $63 \%$ of the cohort and $33 \%$ were transferred to the ICU from their initial medical floor admission. Patients with the outcome of increased organ failure had no difference in initial lactate, age, MAP or exposure to hypotension. Outcome patients had similar initial and 12-hour SOFA scores with higher scores at 24,48 , and 72 hours, a higher ICU transfer rate $(42 \%$ vs. $19 \%, P=0.15)$ and increased ICU ( 2 vs. 0$)$ and hospital length of stays (13 vs. 8). Outcome patients had similar endotoxin levels at enrollment compared with those not meeting outcome (0.61 vs. $0.51, P=$ $0.075)$ with increased levels at 24 hours $(0.73$ vs. $0.47, P=0.005)$. An increasing endotoxin level over 24 hours had a $71 \%$ specificity and $72 \%$ PPV in predicting the primary outcome. Compared with serum lactate, there was no relationship between endotoxin activity and initial serum lactate on linear regression analysis (slope $=0.077, R^{2}=0.0004$ ) .

Conclusion: Within this moderate-severity sepsis cohort, physiologic data in the ED were unable to differentiate those with progressive organ dysfunction over 72 hours, while endotoxin levels were higher at 24 hours in this outcome group. The high specificity and positive predictive value of an increasing endotoxin level as a predictor of progressive organ dysfunction can supplement clinical decision-making to aid clinicians in identifying high-risk patients from this homogeneous population of moderate-severity sepsis patients.

\section{P103}

Defining the impact of delayed antibiotic administration using a comprehensive electronic health record screen to identify sepsis RC Arnold*, SM Hollenberg

Cooper University Hospital, Camden, NJ, USA

Critical Care 2012, 16(Suppl 3):P103

Background: The true effectiveness of early antibiotic administration relative to the clinical identification of sepsis in a real-world setting is unknown. Previous studies described the impact of antibiotic timing within an isolated septic shock cohort. A novel electronic health record (EHR) screening tool, Clinical Vigilance ${ }^{\mathrm{TM}}$ for Sepsis, is able to identify the presence of sepsis and correlate with meaningful patient-centered outcomes. The 
objective was to define the impact of delayed antibiotic administration relative to the clinical identification of sepsis using an EHR alert system.

Methods: A retrospective analysis of a prospectively compiled registry of patient EHR records in a single-center 300-bed community hospital. A consecutive assessment of all adult patients with suspected infection over a 3-month period in 2011. A physician order for intravenous antibiotics was used as a surrogate for the clinical suspicion of systemic infection (sepsis). The test variable was application of a comprehensive automated EHR screening tool, CV Alert, to identify high-risk sepsis patients based on a multifactor alert system including labs, vital signs, and treatment team documentation. The primary outcome was all cause in-hospital mortality, and a secondary outcome was hospital length of stay (LOS). Antibiotic delivery was defined a priori as the time a physician order was placed for intravenous antibiotics and outcomes were assessed every 12 hours prior to and subsequent to the CV Alert.

Results: We identified 2,255 consecutive patients with suspected infection over a 3-month period from a total of 23,717 screened (9.5\%). CV Alert was triggered in 867 of 2,255 (38\%). Patients identified by CV Alert $(n=867)$ had an increased mortality rate $(5.3 \%$ vs. $0.6 \%, P<0.001)$ and increased hospital LOS (5 vs. 2 days, $P<0.001)$ compared with patients not triggering an alert $(n=1,388)$. Patients given antibiotics 0 to 12 hours after the alert had a significantly increased mortality rate $(8.9 \%$ vs. $3.3 \%, P<0.002)$ and longer LOS ( 6 vs. 4 days, $P<0.001)$ compared with patients given antibiotics 0 to 24 hours prior to alert.

Conclusion: Among patients with suspected infection, those identified by the CV Alert had an increased mortality rate and hospital length of stay. Delayed antibiotics relative to the time of CV Alert were associated with a progressive increase in mortality rate and hospital LOS. An EHR-based screening tool applied to a real-time healthcare system could aid in the early identification of at-risk patients within a sepsis cohort.

\section{P104}

Effects on outcome of patients with severe sepsis and septic shock admitted to the ICU after implementation cooperative sepsis management protocol

R Champunot", N Kamsawang, D Thimsri, P Tuandoung, S Tansuphaswasdikul Buddhachinaraj Phitsanulok Hospital, Phitsanulok, Thailand

Critical Care 2012, 16(Suppl 3):P104

Background: The application in clinical practice of evidence-based guidelines for the cooperative management of patients with severe sepsis and septic shock between community hospitals and tertiary referral hospital is still poor. The purpose of this study was to examine the outcome of patients with severe sepsis and septic shock admitted to the ICU after implementation of a cooperative sepsis management protocol.

Methods: From 1 October to 30 November 2009, patients with severe sepsis and septic shock admitted to the ICU received standard therapy (control group). From 1 December 2009 through 31 January 2010, patients with severe sepsis and septic shock (protocol group) were managed with a cooperative sepsis management protocol. The protocol included early recognition and the initiation of therapy by enabling nurses and physicians in community hospitals to mobilize institutional resources for the treatment of patients with severe sepsis and septic shock (Figures 1 and 2). Using goal-directed resuscitation protocols, early intensivist involvement and rapid transfer to the ICU from emergency department were implemented in tertiary referral hospital.

Results: Sixty-two and 77 patients, respectively, were enrolled in the control and protocol groups. Rapid transfer of patients with severe sepsis and septic shock to the ICU from emergency department was observed in $1 / 62(1.5 \%)$ of the control group and $45 / 77(58.4 \%)$ of the protocol group (difference $56.9 \% ; P=0.01$ ). The hospital mortality rate was $62.3 \%$ in the control group and $37.7 \%$ in the protocol group $(P=0.01)$. The protocol group had significant reductions in ICU length of stay, ICU cost and numbers of organ failure $(P=0.01)$.

Conclusion: Empowerment of nurses and physicians in a community hospital to mobilize hospital resources for taking care of patients with severe sepsis and septic shock and implementation of a cooperative sepsis management protocol between community hospitals and tertiary referral hospital was temporally associated with improved outcomes.

Acknowledgements: The authors thank the Phitsanulok Co-operative Sepsis Management Team.

\section{P105}

Saving 500 Lives Campaign: another way to improve the mortality

rate of patients with severe sepsis and septic shock

R Champunot", N Kamsawang, P Tuandoung, S Tansuphaswasdikul

Buddhachinaraj Phitsanulok Hospital, Phitsanulok, Thailand

Critical Care 2012, 16(Suppl 3):P105

Background: In September 2010, 9 months after empowerment of caring for patients with severe sepsis and septic shock and implementation of a cooperative sepsis management protocol between community hospitals and tertiary referral hospital in Phitsanulok, the Phitsanulok Co-operative Sepsis Management (PCSM) team announced the Saving 500 Lives Campaign. This campaign aimed to encourage the unity and importance of caring for patients with severe sepsis and septic shock.

Methods: From October 2010 to September 2011, eight community hospitals and one tertiary referral hospital in Phitsanulok established and promoted a set of achievable goals and interventions for patients with severe sepsis and septic shock (Figure 1). These interventions included: establishing sepsis team in all hospitals to coordinate and monitor the achievement goals; using a search out severity (SOS) score and a sepsis screening tool to help early and more accurate diagnosis; providing early resuscitation protocol and other measures as indicated by implementing a checklist for sepsis management (sepsis six bundles, rule of three, early goaldirected therapy); communication and providing important information during transportation using a protocol; and early intensivist involvement and rapid transfer to the ICU from the emergency department using a sepsis fast-track protocol. Physician and nurse leadership actively engaged in the data review and shared ideas in every hospital for improvement and closely tracked progress against those goals and interventions. The mortality rate of patients with severe sepsis and septic shock was used to measure the success of the campaign.

Results: The Saving 500 Lives Campaign succeeded in efforts to saved 660 patients from 1,048 patients with severe sepsis and septic shock in Phitsanulok, Thailand. The total mortality rate was $37 \%$ (decreased from $47 \%$ in the past year). The group of patients from community hospitals that can be admitted directly to the ICU from the emergency department using a sepsis fast-track protocol had the lowest mortality rate (19\%).

Conclusion: Setting a numeric goal using the 500 Lives Campaign was another way to support and empower the cooperation of sepsis care in Phitsanulok, Thailand.

Acknowledgements: The authors thank the PCSM Team.

References

1. Vincent $J \mathrm{~L}$, Martinez EO, Silva E: Evolving concepts in sepsis definitions. Crit Care Clin 2009, 25:665-675.

2. Schorr C: Performance improvement in the management of sepsis. Crit Care Clin 2009, 25:857-867.

3. Gold JA: The 5 Million Lives Campaign: preventing medical harm in Wisconsin and the nation. WMJ 2008, 107:270-271.

\section{P106}

Clinical utility of using C-reactive protein and procalcitonin as biomarkers for a novel neonatal sepsis diagnostic platform (ASCMicroPlat)

K McAllister $^{1 *}$, M Sheridan-Pereira ${ }^{2}, N^{\prime}$ OSullivan $^{2}$, R O'Kelly $^{2}$, D Mark $^{3}$ G Czilwik ${ }^{3}$, C Martin $^{1}$, O Sheils', J O'Leary

${ }^{1}$ Trinity College Dublin, Ireland; ${ }^{2}$ The Coombe Women and Infants University Hospital, Dublin, Ireland; ${ }^{3}$ HSG-IMIT, Lab-on-a-Chip, Freiburg, Germany Critical Care 2012, 16(Suppl 3):P106

Background: Neonatal sepsis contributes significantly to morbidity and mortality and encompasses a multisystemic inflammatory response to a microorganism. A lack of suitable diagnostic biomarkers and delays in microbe identification (48 to 72 hours) challenge clinical decision-making. Creactive protein (CRP) is the current biomarker for sepsis in patients; however, use of earlier markers such as procalcitonin (PCT) could prove more effective. This study aims to assess the clinical utility of combining CRP and PCT for a novel sepsis diagnostic biomarker platform (ASCMicroPlat).

Methods: Blood samples were obtained from 350 infants evaluated for sepsis at the Coombe Women and Infants University Hospital. Eligible infants met one or more inclusion criteria: age $\leq 6$ weeks, clinical sepsis 


\section{SEVERE SEPSIS and SEPTIC SHOCK Early Recognition for community hospital and Emergency department}

\section{Sepsis : systemic inflammatory response to a documented infection}

\section{SIRS (systemic inflammatory response syndrome)}

severe clinical insults manifests by 2 or more of the following conditions:

-Temperature $>38^{\circ} \mathrm{C}$ or $<36^{\circ} \mathrm{C}$

- Heart rate $>90$ beats $/ \mathrm{min}$

- Respiratory rate $>20 / \mathrm{min}$ หรือ $\mathrm{PaCO} 2<32$ mm Hg

- WBC $>12,000 / \mu \mathrm{L},<4000 / \mu \mathrm{L}$, or $10 \%$ immature (band) forms

$$
\text { Sepsis }=\text { SIRS }+ \text { evidence of infection }
$$

\section{Severe sepsis : sepsis associated with organ dysfunction, hypoperfusion, or hypotension}

\section{Hypotension หมายถึง}

systolic blood pressure $\leq 90$ or MAP $\leq 65 \mathrm{mmHg}$ ( no HT)

systolic blood pressure reduction $\geq 40 \mathrm{~mm} \mathrm{Hg}$ from baseline

$M A P($ mean arterial pressure $)=[(S B P-D B P) / 3]+D B P$

Signs of hypoperfusion may include (but are not limited to)

- acute alteration in mental status

- Oliguria

- capillary refilling time $>2$ seconds

- metabolic acidosis or lactic acidosis

Septic shock: A subset of people with severe sepsis develop hypotension despite adequate fluid resuscitation

Figure 1(abstract P104) Checklist for early recognition patients with severe sepsis and septic shock.

signs, and/or maternal sepsis risk factors. The puncture site was sterilized with alcohol prior to phlebotomy for microbial and biomarker tests. Blood was collected in clotting activator tubes, and incubated at room temperature for 1 hour before centrifuging to separate out serum for later acute-phase protein quantification. For gold-standard tests, an immunoturbimetric CRP assay was performed using a Beckman analyser and a chemiluminescent Elecsys BRAHMS PCT immunoassay using a Cobas e602 analyser.
Results: The mean age of infant evaluation was $2.29 \pm 10.37$ days. The average gestational age at birth was 37.04 weeks (4.27), 30\% of which were preterm. Eighteen out of 350 samples (5\%) were blood culture positive. Thirteen samples met the criteria for a laboratory-confirmed bloodstream infection (LCBSI), the most common organisms being Escherichia coli (28\%) and Streptococcus agalactiae (22\%). CRP results $(n=$ 350 ) ranged from $\leq 0.1$ to $226 \mathrm{mg} / \mathrm{l}$, sepsis-positive ( $\geq 5 \mathrm{mg} / \mathrm{l}$ ) CRP tests occurred in $69 \%$ of LCBSI. PCT quantified samples to date $(n=100)$ 


\section{SEVERE SEPSIS and SEPTIC SHOCK Treatment protocol for community hospital and Emergency department}

-Take Hemoculture 2 specimens -Access peripheral IV 2 lines (no need central venous access) -Retained Foley catheter (and start to record urine output) -Take culture other sites as clinically indicated

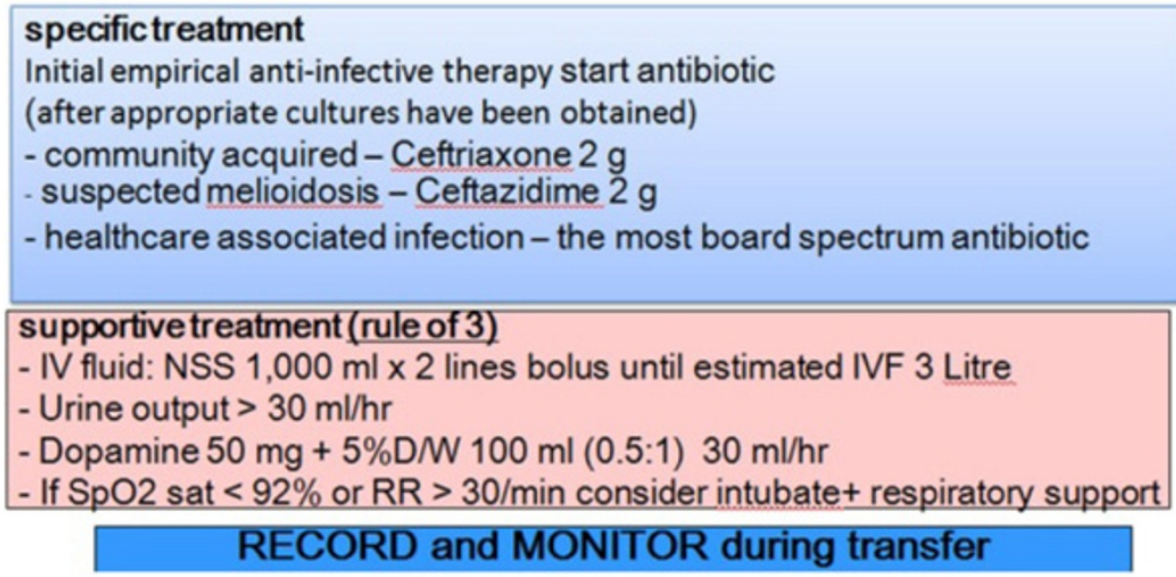

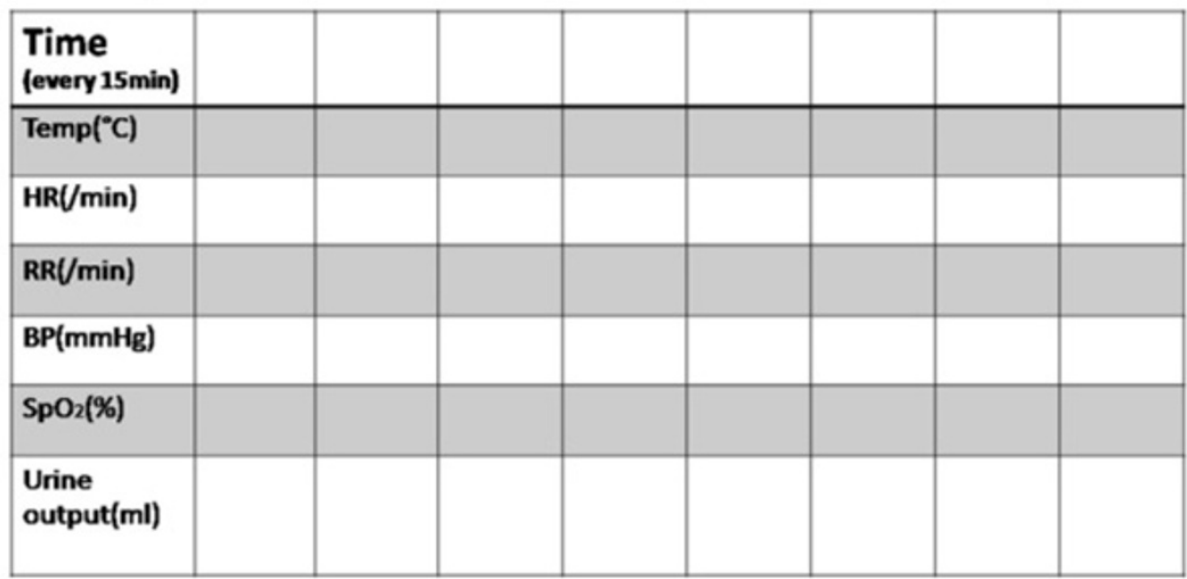

Time start Sepsis Resuscitation

Time reach referal hespital

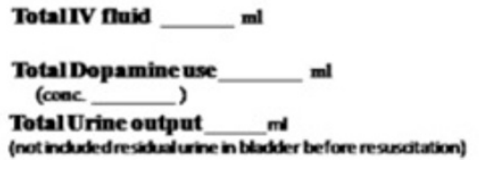

(not indudedresubad unine in bubler before resendation)

Figure 2(abstract P104) Checklist for treatment and transport for severe sepsis and septic shock.

ranged from 0.03 to $67.23 \mathrm{ng} / \mathrm{ml}$. Both analytes featured in higher cases that suggested sepsis than positive blood culture numbers: 56 out of 350 cases had CRP levels $\geq 5 \mathrm{mg} / \mathrm{l}$, for PCT 23 out of 100 individuals tested positive at the cutoff $(>0.5 \mathrm{ng} / \mathrm{ml}$ ) for bacterial infection.

Conclusion: Sepsis diagnosis requires accurate detection biomarkers; preliminary study findings show that CRP is present in the majority of bloodstream infections. Furthermore, results using both biomarkers suggest that blood culture results do not indicate all cases of true sepsis. Further research will correlate the completed CRP and PCT gold-standard dataset with that produced by ASCMicroPlat PCRbased assays to assess the clinical utility of this device for sepsis diagnostics. 


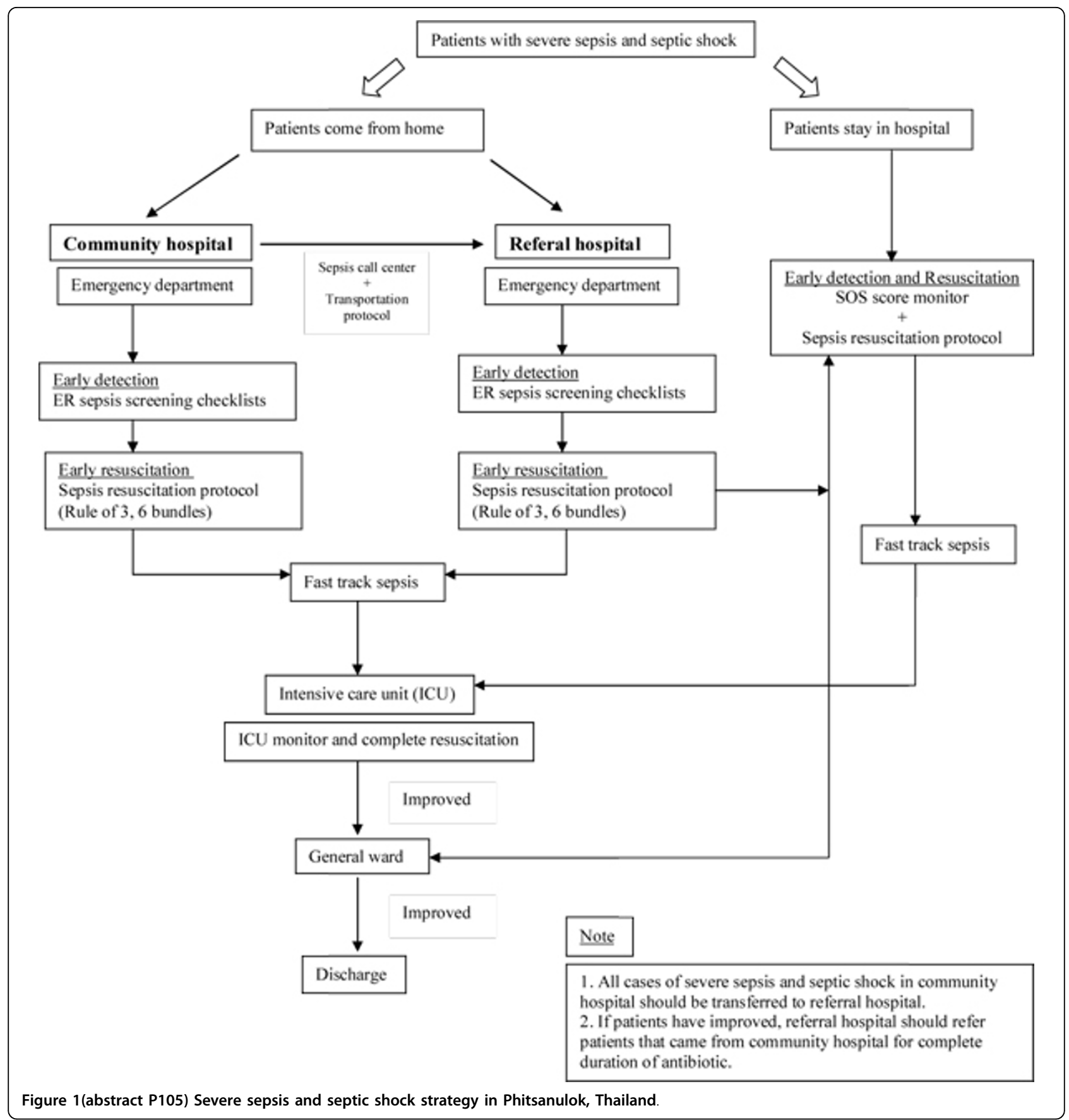

\section{P107}

GAPDH: is it a reliable housekeeper gene to use in sepsis research? J Sarveswaran*, NM Orsi, M Cummings, S Homer-Vanniasinkam, D Burke Leeds Teaching Hospitals, Leeds, UK

Critical Care 2012, 16(Suppl 3):P107

Background: Quantitative gene expression data are often normalised to the expression levels of control genes (that is, housekeeper genes). An assumption in the use of housekeeper genes is that the expression of the genes remains constant in the cells/tissues under investigation. Glyceraldehyde-3-phosphate dehydrogenase (GAPDH) is one of the most commonly used housekeeping genes used in comparisons of gene expression data. Recent studies, however, have shown that GAPDH expression is highly variable in different experimental settings. This study aimed to characterise GAPDH mRNA expression in severe sepsis, using whole blood from patients with severe sepsis and healthy controls.

Methods: Patients admitted to the ICU within 24 hours of onset of severe sepsis were recruited. Healthy controls were also recruited. Exclusion criteria included patients on immunosuppressants or chemotherapy and those with a moribund status. Ethical approval (REC W06/Q1107/42) was obtained. Whole blood $(1 \mathrm{ml})$ was taken from the patients and Quantigene RNA extraction techniques were used. The Quantigene plex assay was used in duplicate to determine GAPDH mRNA expression. The assay uses branched DNA signal amplification to enable the detection and quantification of multiple RNA targets simultaneously. It has been 
compared with other techniques favourably [1]. GAPDH mRNA expression was standardised to housekeeper genes succinate dehydrogenase (SDHA) and hypoxanthine-guanine phosphoribosyltransferase (HPRT).

Results: Sixty-five patients with severe sepsis were included, of whom 27 died within 28 days. Seventeen control samples were included in the study. The groups were age and sex matched. In terms of GAPDH mRNA expression there was no significant difference between survivors and nonsurvivors. However, with regards to overall GAPDH mRNA expression in severe sepsis there was a marked increase in patients with severe sepsis. GAPDH mRNA expression showed a 16-fold mean increase in expression compared with control samples.

Conclusion: GAPDH mRNA expression in patients with severe sepsis showed a marked increase compared with controls. These data question the suitability of GAPDH as a housekeeper gene in gene expression profiling studies in sepsis. The data also highlight GAPDH as another potential biomarker of sepsis that requires further evaluation.

Reference

1. Canales RD, Luo Y, Willey JC, Austermiller B, Barbacioru CC, Boysen C, Hunkapiller K, Jensen RV, Knight CR, Lee KY, et al: Evaluation of DNA microarray results with quantitative gene expression platforms. Nat Biotechnol 2006, 24:1115-1122.

\section{P108}

Cytokine gene expression profiling identifies distinct patterns in severe sepsis

J Sarveswaran*, NM Orsi, M Cummings, S Homer-Vanniasinkam, D Burke Leeds Teaching Hospitals, Leeds, UK Critical Care 2012, 16(Suppl 3):P108

Background: In patients with severe sepsis, previous work has suggested that cytokine gene expression profiles may have diagnostic/prognostic potential as biomarkers. Cytokines operate within complex networks; their predictive value may be improved by the simultaneous measurement of multiple cytokines. Cytokines are key inflammatory proteins involved in sepsis. Gene expression profiling has been shown to have a clinical role in the diagnosis of haematological malignancies. Gene expression profiling may allow early, aggressive management in patients predicted to fare badly. This study examined cytokine gene expression profiles in patients with severe sepsis.

Methods: Patients admitted to the ICU within 24 hours of onset of severe sepsis were recruited. Healthy controls were also recruited. Exclusion criteria included patients on immunosuppressants or chemotherapy and those with a moribund status. Ethical approval (REC W06/Q1107/42) was obtained. Whole blood $(1 \mathrm{ml})$ was taken from the patients and Quantigene RNA extraction techniques were used. The following cytokines were measured in duplicate using the Quantigene plex assay: IL-6, IL-8, IL-10, IL-12p40, IL12p35, IL-13, IL-17, MCP-1 and MIF. The assay uses branched DNA signal amplification to enable the detection and quantification of multiple RNA targets simultaneously. It has been compared with other techniques favourably [1]. Cytokine mRNA expression was standardised to housekeeper genes succinate dehydrogenase (SDHA) and hypoxanthine-guanine phosphoribosyltransferase (HPRT).

Results: Sixty-five patients with severe sepsis were included, of whom 27 died within 28 days. Seventeen control samples were included in the study. The groups were age and sex matched. Three cytokines showed marked upregulation in severe sepsis compared with controls; IL-10 (15-fold), IL-18 (15-fold), IL-15 (twofold). However, IL-6, IL-8, IL-12p40, IL-12p35, IL-13, IL-17, MCP-1 all showed a greater than twofold downregulation in severe sepsis. There was no significant difference in profiles between survivors and nonsurvivors of severe sepsis.

Conclusion: These results show a distinct cytokine gene expression profile in severe sepsis. This includes the upregulation or downregulation of both proinflammatory and anti-inflammatory cytokines. Cytokine gene expression profiling using the Quantigene plex assay is able to demonstrate distinct profiles in patients with severe sepsis. This has the potential to be developed into a diagnostic/prognostic tool with larger studies.

Reference

1. Canales RD, Luo Y, Willey JC, Austermiller B, Barbacioru CC, Boysen C, Hunkapiller K, Jensen RV, Knight CR, Lee KY, et al: Evaluation of DNA microarray results with quantitative gene expression platforms. Nat Biotechnol 2006, 24:1115-1122.

\section{P109}

Quantified temporal changes of heart rate variability when

developing SIRS

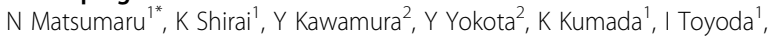

S Ogura ${ }^{1}$

Advanced Critical Care Center, Gifu University Hospital, Gifu, Japan; ${ }^{2}$ Faculty of Engineering, Gifu University, Gifu, Japan

Critical Care 2012, 16(Suppl 3):P109

Background: Heart rate variability (HRV) has been reported to gradually decrease long before the onset of sepsis [1,2]. Since the development of systemic inflammatory response syndrome (SIRS) has been included in the definition of sepsis, we compared temporal changes of HRV depending on the SIRS state coming after.

Methods: Vital data of patients admitted in ICU were downloaded from bedside monitoring systems. No specific registration criteria for disease were defined. Vital signs regarding SIRS evaluation - that is, hear rate, respiration rate, and core temperature - are recorded every 10 seconds, $0.1 \mathrm{~Hz}$. By taking the median of 60 data, the SIRS state was determined for each hour, ignoring the count of white blood cells. The sampling rate of ECG data is $500 \mathrm{~Hz}$, and the HRV for each minute $(0.017 \mathrm{~Hz})$ was calculated from that. Abnormal heartbeats and trends were eliminated using a stochastic model, and the power spectrum was derived with an autoregressive model [3]. We considered the integral value within 0.04 to $0.4 \mathrm{~Hz}$ as an estimate of HRV. Temporal linear trends of HRV were quantified as Spearman's correlation coefficient. Note that the target period for HRV trend evaluation is immediately before the SIRS evaluation period. The following software was used: IBM SPSS version 19, R version 2.15.0, and G*Power Version 3.1.4.

Results: Forty-nine patients were registered, and 34 patients (69.4\%) suffered from sepsis. The average age was 64.3 years, and the average ICU stay was 31.4 days, ranging from 3 to 113 days. The number of data where a SIRS state was identified was 21,919 , and $56.5 \%(12,380)$ were evaluated as SIRS-positive. Immediately before SIRS-positive states, the mean value of the correlation coefficients was -0.00234 (SD 0.321). The mean value before SIRSnegative states was 0.0201 (SD 0.309). The mean difference was $0.0224(P<$ $0.05)$, and the statistical power was $99.9 \%$.

Conclusion: Our analysis shows that the mean trend of the HRV value immediately before developing SIRS was negative, and the mean trend of HRV immediately before a non-SIRS state was positive. This result does support previous results. For sepsis monitoring, however, further investigation is mandatory. This result only suggests a stronger negative trend of HRV when developing SIRS for the next period, regardless of the current state.

Acknowledgements: Supported by the Ministry of Education, Culture, Sports, Science and Technology Japan as a part of Regional Innovation Cluster Program (City Area Type) in Southern Gifu Area.

References

1. Moriguchi T, Hirasawa H, Oda S, Tateishi Y: [Analysis of heart rate variability is a useful tool to predict the occurrence of septic shock in the patients with severe sepsis]. Nihon Rinsho 2004, 62:2285-2290.

2. Ahmad S, Ramsay T, Huebsch L, Flanagan S, McDiarmid S, Batkin I, McIntyre L, Sundaresan SR, Maziak DE, Shamji FM, Hebert P, Fergusson D, Tinmouth A, Seely AJE: Continuous multi-parameter heart rate variability analysis heralds onset of sepsis in adults. PLoS One 2009, 4:e6642.

3. Yokota Y, Kawamura Y, Matsumaru N, Shirai K: Premonitory symptom of septic shock in heart rate variability. 5th Kuala Lumpur International Conference on Biomedical Engineering 2011. Volume 35 of IFMBE Proceedings Berlin: Springer: Osman NAA, Abas WABW, Wahab AKA, Ting HN, Magjarevic R 2011, 552-555.

\section{P110}

Endothelial cell specific molecule 1 is today a relevant marker of respiratory failure in sepsis and polytrauma patients

P Lassalle 1* $^{*}$ N De Freitas Caires ${ }^{1}$, L Portier ${ }^{1}$, A Palud $^{2}$, E Parmentier $^{2}$, J Pastre $^{2}$, C Shah $^{3}$, A Scherpereel ${ }^{4}$, D Mathieu ${ }^{2}$, M Delehedde

${ }^{1}$ Lunginnov, R\&D Departement, Lille, France; ${ }^{2} \mathrm{CU}$, Calmette Hospital, Lille, France; ${ }^{3}$ University, Philadelphia, PA, USA; ${ }^{4}$ Service de Pneumologie, Calmette Hospital, Lille, France

Critical Care 2012, 16(Suppl 3):P110 
Background: Initially described in endothelial cells from the lung, endothelial cell specific molecule 1 (ESM-1), also called endocan, is a circulating proteoglycan of $50 \mathrm{kDa}$ that can be easily quantified in biological fluids (sera, plasma). This robust macromolecule is upregulated in the presence of proinflammatory agents and has been described as a biomarker of endothelial dysfunction in several disease conditions.

Methods: We here compared and analyzed the results obtained from studies using a sandwich-type ELISA (DIYEK H1, Lunginnov) for evaluating whether ESM-1 could be useful to predict organ failure in ICU patients such as septic patients and polytrauma patients $(n=67)$. Data baseline characteristics, APACHE II score, procalcitonin (PCT) and C-reactive protein (CRP) levels and ICU course such as ICU length of stay were also evaluated for each ICU patient admitted and compared with ESM-1 levels.

Results: In all studies we observed that elevated blood levels of ESM-1 correlated with the severity of sepsis and the poor outcome in patients with severe sepsis or in septic shock at ICU admission. We furthermore evaluated the presence of ESM-1 and ESM-1 degradation products in the biological fluids (sera, plasma and urines) from septic patients by ELISA, immunoprecipitation and western blotting procedures. When compared with data baseline characteristics, ESM-1 levels were shown to not correlate with CRP and PCT levels. Interestingly we may suggested from our analysis that blood ESM-1 $>3 \mathrm{ng} / \mathrm{ml}$ represents an additional criterion of severity in a context of SIRS that may be useful in the ICU. At 72 hours, ESM-1 exhibited a clear predictive value for acute lung injury (sensitivity $85 \%$; specificity $100 \%$ ) in septic patients. In comparison, lower levels of serum ESM-1 in polytrauma patients were associated with development of acute lung injury and reflect respiratory failure.

Conclusion: No circulating molecule was up to now described as an indicator of respiratory failure in septic patients and in polytrauma patients. In a context where respiratory failure is still the first cause of death in sepsis, our study analysis suggests that blood levels of ESM-1 may be a useful early biomarker of lung tissue injury and respiratory failure in ICU patients.

\section{P111}

A microbiome approach to sepsis: development and case-study application of novel methods for detection and isolation of microbes from whole blood

M Faria Crowder ${ }^{1 *}$, JM Conly ${ }^{2}$, MG Surette ${ }^{3}$

${ }^{1}$ University of Calgary, Calgary, Canada; ${ }^{2}$ University of Calgary, Snyder Institute for Chronic Diseases, Calgary, Canada; ${ }^{3}$ McMaster University, Farncombe Family Digestive Health Research Institute, Hamilton, Canada Critical Care 2012, 16(Suppl 3):P111

Background: The application of molecular profiling methods to a wide variety of infections suggests that a polymicrobial community is much more common than suggested by standard clinical culture [1]. Our goal was to develop methods, using a microbiome approach, to improve culture and molecular diagnostics for bacterial sepsis.

Methods: Culture and DNA extraction protocols were evaluated using synthetic bacterial communities inoculated into whole blood. Disruption of blood cells with a blood cell lysing detergent, with or without hypotonic osmotic shock, was carried out and evaluated for the ability to recover the community. Viable bacterial cells were recovered on solid media. Total DNA was examined by terminal-restriction fragment-length polymorphism (TRFLP) profiling. Efficiencies of recovery and limits of detection were determined. The optimized methodology was applied to clinical samples collected from consented patients in both the ICU and ED from two Calgary hospitals. Cultured organisms were identified by $16 \mathrm{~S}$ rRNA gene sequencing. Molecular profiling was carried out using TRFLP and bacterial tag-encoded FLX amplicon pyrosequencing (bTEFAP).

Results: Treatment of synthetic community organisms with a $5 \% \mathrm{wt} / \mathrm{vol}$ detergent added at a 1:1 or 1:5 ratio did not significantly impact their viability. TRFLP analysis indicated that the DNA from these communities could be recovered from whole blood following lysis and removal of host cells. Whole blood samples were analysed from septic patients. In three case studies we identified two to 16 bacterial species in the primary infection samples using direct culture and molecular methods. Conventional diagnostics only reported one organism. Molecular profiling of blood samples from these patients also identified correlating polymicrobial communities. Blood cultures for these samples were either negative (two of three cases) or monomicrobial (one of three cases), thereby underestimating the diversity seen with the TRFLP and bTEFAP analysis. These methods have been applied to 88 adult ICU blood samples, 20 primary infection samples, 36 adult ED samples, and seven pediatric ED samples with analysis ongoing. Conclusion: We have successfully developed a novel method to analyse whole blood in order to characterize the microbiome of sepsis infections. Preliminary results indicate that sepsis infections are polymicrobial in nature.

Acknowledgements: All samples were collected in collaboration with the Alberta Sepsis Network through the Critical Care Epidemiologic and Biologic Tissue Resource.

\section{Reference}

1. Sibley CD, Church DL, Surette MG, Dowd SE, Parkins MD: Pyrosequencing reveals the complex polymicrobial nature of invasive pyogenic infections: microbial constituents of empyema, liver abscess, and intracerebral abscess. Eur J Clin Microbiol Infect Dis 2012 in press.

\section{P112}

Antibacterial therapy in treatment of newborns with perinatal sepsis $\mathrm{G} \mathrm{Khanes}^{1^{*},}$ S Bidnenko ${ }^{2}, \mathrm{O}$ Liutko $^{2}$

${ }^{1}$ Ukrainian National Paediatric Hospital OkhMatDyt, Kyiv, Ukraine; ${ }^{2}$ Ukrainian National Orthopedic Institute, Kyiv, Ukraine

Critical Care 2012, 16(Suppl 3):P112

Background: Perinatal sepsis is the leading problem for morbidity and lethality of children under 1 year old. Antibacterial therapy from the very first hours of decease development plays an important role in the treatment of perinatal sepsis. To determine the adequacy of antibacterial therapy of perinatal sepsis during 18 years we examined the etiology of perinatal sepsis in two groups of newborns with surgical pathology.

Methods: There were 114 newborns in the first group, from 1992 to 2007. We determined the antibodies in blood to pathogenic microflora extracted from joint bursas and in 52 newborns - extracted from the abdominal cavity with peritonitis. These investigations were made using the agglutination reaction to staphylococci as well as the latex-based test for streptococci. The antibiotics were prescribed according to the results of the investigations. In the second group from 2005 to 2010, we undertook microbiological investigations of pathological material from lesion foci in 43 newborns with surgical sepsis. These investigations are devoted to the study of susceptibility and resistance of pathogenic microflora to antibiotics.

Results: In the first group of infants, according to the data of serological investigations in the blood of newborns with bone and joint sepsis, we determined the antibodies to staphylococci in $80.3 \%$ cases, in $45.4 \%$ to staphylococci and streptococci, in $54 \%$ cases in newborns with peritonitis we determined the antibodies to Pseudomonas aerogenes, in $60.8 \%$ cases to Enterobacter spp. and in $62.5 \%$ to Staphylococcus epidermidis. In newborns of this group the bacterial flora were $70 \%$ and fungal in $30 \%$. Our investigations determined the susceptibility to the limited range of antibacterial and antifungal medicines. In the second group we selected two subgroups of infants: one with sepsis as a complication of surgical deceases, and the second with bone and joint sepsis. In the first subgroup the bacterial microflora was detected in $94.7 \%$ of examined newborns and represented in $52.6 \%$ as microbial associations. In the second subgroup of newborns the bacterial flora was detected in $37.5 \%$ of cases, in the rest of the cases the flora consisted of fungi and protozoa. The analysis of susceptibility to pathogenic microflora showed that two of seven cultures of staphylococcus were methicillin-resistant Staphylococcus aureus, in five of eight cultures of staphylococcus they were resistant to four to 10 antibiotics, and the highest resistance to the antibiotics was detected in $P$. aerogenes (in nine of 18 cultures).

Conclusion: Microbiological and serological diagnostics are a compulsory condition of adequate anti-inflammatory therapy for perinatal sepsis.

\section{P113}

Use of intravenous and intramuscular immunoglobulin in the practice of treatment for purulent and septic deaths in newborns $\mathrm{G} \mathrm{Khanes}^{1 *}$, L Nazarchuk ${ }^{2}$, I Maksakova', O Bakaieva ${ }^{1}$

${ }^{1}$ Ukrainian National Paediatric Hospital OkhMatDyt, Kyiv, Ukraine; ${ }^{2}$ Ukrainian Research Institute of Hematology and Transfusiology, Kyiv, Ukraine Critical Care 2012, 16(Suppl 3):P113 
Background: The investigations of A Fedorovska and L Nazarchuk (1995 to 2000) showed the necessity to introduce specific antigens of $G$ class for the treatment of sepsis in immunodeficiency patients. We refer newborns to such patients. Effective treatment of sepsis in newborns and children of a young age group is impossible without evaluation of immunocompetent systems.

Methods: We successfully used intravenous normal human immunoglobulin Bioven mono when extracting anti-staphylococcus, anti-proteus and anti-pseudomonas antibodies in 150 newborns with postoperative and bone and joint sepsis. This immunoglobulin has international certification. During the last 10 years the main problem for treatment of postoperative sepsis in newborns is the treatment of pseudomonade infection. Regarding that traditional preparations have an insignificant titer of anti-pseudomonade antibodies, we used new anti-pseudomonade immunoglobulin received from specific plasma for 37 newborns and children under 1 year old. There were 20 patients having sepsis with esophageal atresia, two patients with angiocardiogenic sepsis, five with laryngotracheostenosis, five with bone and joint sepsis, and five after polytrauma with injury of pelvis bones and urinary bladder. In these 37 patients we used a new method for introduction of immunoglobulin in combination with fresh frozen plasma (patented).

Results: Use of Bioven in combination with antibacterial therapy allowed treatment of up to $90 \%$ of infants with severe sepsis. The use of new anti-pseudomonade immunoglobulin allowed recovery of the susceptibility of pathogenic flora to antibiotics after triple introduction of specific immunoglobulin, to raise phagocytal response and thus to gain positive results in 35 patients.

Conclusion: The experience of immune system correction in newborns with postoperative and bone and joint sepsis allow one to consider this method a leading one in clinic practice. The use of specific immune preparations increases complementary activity of blood and antibiotic susceptibility of pathogenic microflora.

\section{P114}

An overview of the sepsis situation in the Department of Infection Diseases, University Hospital Center, Tirana

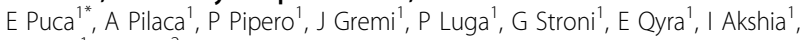
D Kraja', E Puca

'University Hospital Center 'Mother Teresa', Tirana, Albania; ${ }^{2}$ American

Hospital, Tirana, Albania

Critical Care 2012, 16(Suppl 3):P114

Background: Sepsis is a condition that has been known since 2,700 years ago, but it is still a serious threat for human health [1]. Sepsis is a serious public health problem [2]. It is a complex process that can affect any individual and can originate from multiple sites and be caused by multiple microorganisms [3].

Methods: We conducted a retrospective, observational study at University Hospital Center, Tirana, involving 535 critically ill patients diagnosed with sepsis admitted to the Department of Infection Diseases from January 2006 to December 2011. Blood cultures were performed on the admission day.

Results: During the study period there were 535 patients with sepsis admitted to our hospital. From these, $288(53.83 \%)$ were males and 247 $(46.16 \%)$ were females. In the ICU, 103 cases (19.25\%) with diagnosis of sepsis or severe sepsis were admitted. The urinary tract was the predominant site of infections in $39.81 \%$ of cases, followed by the lungs in $34.95 \%$ of cases, intra-abdominal infection (not postoperative) in $15.7 \%$ of cases, soft tissues in $2.24 \%$ of cases and with unknown origin in $7.28 \%$ of cases. Organ failure (severe sepsis or multiorgan dysfunctions) was found in $37.9 \%$ of patients. Blood culture results were positive in $23 \%$ of the patients. See Table 1.

Conclusion: Our study shows a progressive increase in the incidence of sepsis mostly in the last years. This could be explained by the possibility of increasing cases with sepsis or the doctors being affiliated with sepsis terminology. Urinary tract infection was the leading cause of sepsis. These data could be explained because our hospital is not the only center for treatment of sepsis and in our country we have another hospital for the treatment of lung diseases. The low value of positive culture results could be explained by our health system problems, using antibiotics much more than needed or antibiotic therapy being started before admission to our hospital. However, we propose that a developing country like Albania needs to develop a national center for sepsis.

References

1. Yang $Y$, Yang KS, et al: The effect of comorbidity and age on hospital mortality and length of stay in patients with sepsis. J Crit Care 2010, 25:398-405.

2. Carlos J, Ballester A, Ballester F, et al: Epidemiology of sepsis in the Valencian community (Spain), 1995-2004. Infect Control Hosp Epidemiol 2008, 29:630-634.

3. Vincent JL, Martinez EO, Silva E: Evolving concepts in sepsis definitions. Crit Care Clin 2009, 25:665-675.

\section{P115}

Is visceral leishmaniasis a sepsis or not?

E Puca $^{1 *}$, P Pipero 1 , P Pilaca', E Puca ${ }^{2}$

'University Hospital Center 'Mother Teresa', Tirana, Albania; ${ }^{2}$ American

Hospital, Tirana, Albania

Critical Care 2012, 16(Suppl 3):P115

Background: Based on a sepsis consensus conference in 1992 and after that in 2001, sepsis is defined as a syndrome by the presence of both infection and a systemic inflammatory response (SIRS). SIRS is considered to be present when patients have more than one of the following clinical findings: body temperature $>38^{\circ} \mathrm{C}$ or $<36^{\circ} \mathrm{C}$; heart rate $>90 /$ minute; hyperventilation evidenced by a respiratory rate $>20$ /minute or $\mathrm{PaCO}_{2}$ $<32 \mathrm{mmHg}$; and white blood cell count $>12,000$ cells/ $\mu \mathrm{l}$ or $<4,000 / \mu \mathrm{l}[1]$. On the other hand visceral leishmaniasis (VL), or kala-azar, is a parasitic disease that consists of a protracted course of fever, pallor, wasting, hepatosplenomegaly and pancytopenia [2,3]. The aim of our study is to start a discussion about $\mathrm{VL}$ and sepsis.

Methods: From 45 adults patients diagnosed with VL between January 2005 and December 2009 in the Service of Infectious Diseases, University Hospital Centre of Tirana, Albania, we have selected 36 patients who presented with fever $>38^{\circ} \mathrm{C}$ and leucopenia $<4,000 / \mu \mathrm{l}$. The diagnosis of $V L$ was based on demonstration of leishmania parasites in bone marrow smears.

Results: From 36 patients $58.3 \%$ were males and 41.6 were females. Age ranged from 17 to 69 years, the average age was 43.2 years. Main clinical and laboratory findings were: fever in $100 \%$, malaise in $100 \%$, hepatosplenomegaly in $100 \%$, anemia in $82 \%$, leucopenia in $100 \%$, thrombocytopenia in $50 \%$, and increased liver enzymes in $52.7 \%$ of cases. Bone marrow aspirate was performed in all cases with amastigotes identified in $100 \%$ of the cases. Meglumine antimoniate was used in all cases as an initial treatment. Treatment failure occurred in two cases $(5.5 \%)$ that were treated subsequently with liposomal amphotericin B. The case fatality was $5.5 \%$. The main causes of death were liver and cardiac failure.

Table 1(abstract P114) Distribution of cases with sepsis in department of infection diseases during 2006 to 2010

\begin{tabular}{llllllll}
\hline & $\mathbf{2 0 0 6}$ & $\mathbf{2 0 0 7}$ & $\mathbf{2 0 0 8}$ & $\mathbf{2 0 0 9}$ & $\mathbf{2 0 1 0}$ & $\mathbf{2 0 1 1}$ & Total \\
\hline Cases & 59 & 57 & 63 & 76 & 122 & 158 & 535 \\
Males & 34 & 35 & 27 & 41 & 69 & 82 & 288 \\
Females & 25 & 22 & 36 & 35 & 53 & 76 & 247 \\
Mean age & 44.28 & 47.59 & 44.46 & 40.56 & 48.88 & 49.92 & 45.94 \\
Standard deviation & 18.98 & 18.96 & 19.18 & 18.77 & 20.03 & 19.34 & 19.21 \\
\hline
\end{tabular}


Conclusion: Based on the clinical and laboratory findings VL could be considered a systemic inflammatory response. Maybe in this disease we have not evaluated an inflammatory test like C-reactive protein or procalcitonin to be convicted if higher than the normal value, but we think we need to start a study about markers of sepsis in this systemic parasitic disease, because our opinion is that VL is a SIRS plus parasitic systemic infection - which does mean sepsis. In malaria, PCT levels are elevated in both severe and uncomplicated Plasmodium falciparum malaria, but how useful is this inflammatory test in VL [4]?

\section{References}

1. Levy MM, Fink MP, et al: SCCM/ESICM/ACCP/ATS/SIS: 2001 SCCM/ESICM/ ACCP/ATS/SIS International Sepsis Definitions Conference. Crit Care Med 2003, 31:1250-1256.

2. Costa $\mathrm{CH}$, Werneck $\mathrm{GL}$, et al: Is severe visceral leishmaniasis a systemic inflammatory response syndrome? A case control study. Rev Soc Bras Med Trop 2010, 43:386-392.

3. Miranda de Arau VE, Morais MHF: Early clinical manifestations associated with death from visceral leishmaniasis. PLoS Negl Trop Dis 2012, 6:e1511.

4. Jin M, Khan Al: Procalcitonin: uses in the clinical laboratory for the diagnosis of sepsis. LABMEDICINE 2010, 41:173-177.

\section{P116}

Evaluation of procalcitonin in patients with sepsis in Albanian adults

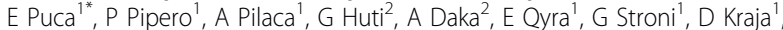

E Puca $^{2}$

'University Hospital Center 'Mother Teresa', Tirana, Albania; ${ }^{2}$ American

Hospital, Tirana, Albania

Critical Care 2012, 16(Suppl 3):P116

Background: Sepsis is a systemic response to infection by microbial organisms [1]. However, differentiating sepsis from non-infectious triggers of the systemic inflammatory response syndrome (SIRS) is difficult. One promising marker is procalcitonin (PCT), whose concentration has been found to be elevated in sepsis [1-3]. The traditional clinical signs and the routine laboratory tests for sepsis, such as C-reactive protein (CRP) or leukocyte count, lack diagnostic accuracy and are sometimes misleading [1]. The use of biomarkers provides a novel, complementary approach to diagnose infection. In most infections, a true gold standard for diagnosis does not exist, thus the measurement of biomarkers, specifically the hormokine PCT in a clearly defined setting, has been shown to significantly improve the diagnostic certainty [3]. The aim of the study was to assess the value of white blood cell count (WBC), CRP, and PCT for the diagnosis of SIRS and sepsis. In Albania, PCT has been used as a serum marker for bacterial sepsis since May 2010.

Methods: We evaluated WBC, CRP, and PCT in 143 adult patients presented to the Service of Infection Diseases, University Hospital Center 'Mother Teresa' and the American Hospital, Tirana, Albania. We used the Elycsys BRAHMS PCT test Electro Chemiluminescent Immunoassay (ECLIA) for the determination of PCT. We evaluated those parameters in three subgroups: 59 patients with sepsis, 64 patients with SIRS, and 20 patients as a control group. The cutoff of PCT for sepsis positivity was $\geq 2 \mathrm{ng} / \mathrm{ml}$. Blood cultures were performed on the admission day or on the day of fever onset for the hospitalized patients.

Results: In the sepsis subgroup the mean \pm SD age was $53.4 \pm 22.8$ years. Average values of PCT, PCR and WBC were respectively $17.8 \mathrm{ng} / \mathrm{ml}, 37.3$ $\mathrm{ng} / \mathrm{l}$ and $13.7 \times 10^{3}$ cells $/ \mathrm{mm}^{3}$. We excluded a case with septic shock with PCT value $761 \mathrm{ng} / \mathrm{l}$. In SIRS subgroups the mean age was $46.5 \pm 18.3$ years and the average value of PCR and WBC were $21.3 \mathrm{ng} / \mathrm{l}$ and $11.6 \times 10^{3}$ cells $/ \mathrm{mm}^{3}$, but PCT was $\leq 2 \mathrm{ng} / \mathrm{ml}$. In the control group the mean age was $44.3 \pm 25.24$ years, and $\mathrm{WBC}, \mathrm{PCR}$ and PCT were in normal values (respectively 3.5 to $10 \times 10^{3}$ cells $/ \mathrm{mm}^{3}, 0$ to $5 \mathrm{ng} / \mathrm{l}$ and 0.5 to $2 \mathrm{ng} / \mathrm{ml}$ ). Blood culture resulted positive in $40.6 \%$ of patients with sepsis.
Conclusion: The serum concentration of PCT is specifically elevated in patients with sepsis. However, the evidence of PCT in infectious diseases is limited. The diagnostic model based on the laboratory parameters, using the combined predictors of PCT, CRP and WBC, can be a useful means for predicting early onset of sepsis.

\section{References}

1. Jin M, Khan Al: Procalcitonin: uses in the clinical laboratory for the diagnosis of sepsis. LABMEDICINE 2010, 41:173-177.

2. Kibe S, Adams K, Barlow G: Diagnostic and prognostic biomarkers of sepsis in critical care. J Antimicrob Chemother 2011, 66:33-40.

3. Muñoz P, Simarro N, Rivera M, et al: Evaluation of procalcitonin as a marker of infection in a nonselected sample of febrile hospitalized patients. Diagnostic Microbiol Infect Dis 2004, 49:237-241.

\section{P117}

Role of the membrane receptor ALXR in polymicrobial sepsis

LC Resende Silva*, ID Tavares, MM Teixeira, D Da Gloria De Souza

ICB/UFMG, Belo Horizonte, Brazil

Critical Care 2012, 16(Suppl 3):P117

Introduction: Sepsis is a leading cause of death in the ICU. It is characterized by a systemic inflammatory response following bacterial infection. Inflammatory dysregulation affects multiple organs through effects on endothelial, epithelial and immune cells, which can lead to irreversible damage and death of the host. Although exacerbated inflammation plays a pivotal role in sepsis evolution, anti-inflammatory drugs fail to treat this disease. In addition, anti-inflammatory therapy may inhibit clearance of infection or favor secondary infections. Therefore, it is of interest to resolve excessive inflammation without compromising host defense mechanisms. In accordance, it has been shown that treatment with LXA4, a endogenous anti-inflammatory agent with pro-resolution activity, increased survival in experimental sepsis by reducing systemic inflammation as well as bacterial spread. We therefore aimed to evaluate the role of a nonspecific LXA4 receptor, ALXR, in host response to sepsis induction by cecal ligation and puncture in mice (CLP).

Methods: To assess the role of ALXR in the CLP model we used WT (C57BL/ 6) mice treated or not with an ALXR antagonist, BOC-1 (2 mg/kg, i.v.), 30 minutes before sepsis induction. Then, 6 hours after mild and severe sepsis induction, we evaluated the pulmonary damage, bacterial spread, neutrophil recruitment and the levels of cytokines, chemokines in the lung, peritoneal exudate and plasma.

Results: Animals submitted to mild and severe sepsis induction and treated with BOC-1 presented decreased neutrophil migration, larger bacterial loads in peritoneal exudate, and heightened lung damage when compared with untreated control animals. Interestingly, ALXR blockade did not alter levels of cytokines and chemokines such as IL-6, IL-1 $1 \beta$, TNF $\alpha$ and $K C$ in all evaluated tissues.

Conclusion: Animals treated with the antagonist of ALXR receptor demonstrated a worse outcome after sepsis induction when compared with animals that received vehicle. These data suggest that the membrane receptor ALXR plays a protective role during response to sepsis induced by CLP.

Acknowledgements: Financial support from CAPES, FAPEMIG and CNPq.

Cite abstracts in this supplement using the relevant abstract number, e.g.: Silva et al:: Role of the membrane receptor ALXR in polymicrobial sepsis. Critical Care 2012, 16(Suppl 3):P117 